\title{
IntechOpen
}

\section{Clinical and Genetic Aspects of Epilepsy}

\author{
Edited by Zaid Afawi
}





\section{CLINICAL AND GENETIC ASPECTS OF EPILEPSY}

Edited by Zaid Afawi 


\section{Contributors}

Wen-Hsing Cheng, Inga I. Poletaeva, Natalia M. Surina, Zoya A. Kostyna, Irina B. Fedotova, Stella Tsirka, Martine M Mirrione, Elisabeth Ngo Bum, Germain Sotoing Taïwe, Fleur Moto, Gwladys Temkou Ngoupaye, Espoir Ayissi, Christian Dong, Frédéric Maidawa, Gildas Djafsia, Leopold Nanga, Saleh Soudi, Alice Rakotonirina, Silvère Rakotonirina, Kaneez Fatima Shad, Faisal Khan, Sarah Heron, John Mulley, John Cressman, Christine Drown, Chin-Wei Huang, Bjoern Bauer, Anika Maria Sophie Hartz, Juli Schlichtiger, Anton Pekcec

\section{(c) The Editor(s) and the Author(s) 2011}

The moral rights of the and the author(s) have been asserted.

All rights to the book as a whole are reserved by INTECH. The book as a whole (compilation) cannot be reproduced, distributed or used for commercial or non-commercial purposes without INTECH's written permission.

Enquiries concerning the use of the book should be directed to INTECH rights and permissions department (permissions@intechopen.com).

Violations are liable to prosecution under the governing Copyright Law.

\section{(c) BY}

Individual chapters of this publication are distributed under the terms of the Creative Commons Attribution 3.0 Unported License which permits commercial use, distribution and reproduction of the individual chapters, provided the original author(s) and source publication are appropriately acknowledged. If so indicated, certain images may not be included under the Creative Commons license. In such cases users will need to obtain permission from the license holder to reproduce the material. More details and guidelines concerning content reuse and adaptation can be foundat http://www.intechopen.com/copyright-policy.html.

\section{Notice}

Statements and opinions expressed in the chapters are these of the individual contributors and not necessarily those of the editors or publisher. No responsibility is accepted for the accuracy of information contained in the published chapters. The publisher assumes no responsibility for any damage or injury to persons or property arising out of the use of any materials, instructions, methods or ideas contained in the book.

First published in Croatia, 2011 by INTECH d.o.o.

eBook (PDF) Published by IN TECH d.o.o.

Place and year of publication of eBook (PDF): Rijeka, 2019.

IntechOpen is the global imprint of IN TECH d.o.o.

Printed in Croatia

Legal deposit, Croatia: National and University Library in Zagreb

Additional hard and PDF copies can be obtained from orders@intechopen.com

Clinical and Genetic Aspects of Epilepsy

Edited by Zaid Afawi

p. cm.

ISBN 978-953-307-700-0

eBook (PDF) ISBN 978-953-51-6489-0 


\section{We are IntechOpen, \\ the world's leading publisher of Open Access books}

Built by scientists, for scientists

\section{$4,100+$}

Open access books available

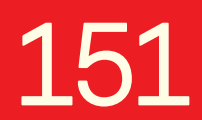

Countries delivered to
$116,000+$

International authors and editors
$120 \mathrm{M}+$

Downloads

Our authors are among the

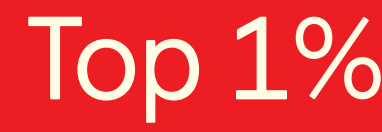

most cited scientists

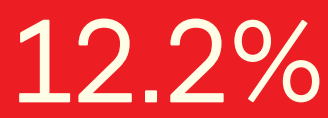

Contributors from top 500 universities

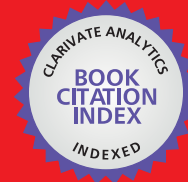

WEB OF SCIENCE ${ }^{\mathrm{TM}}$

Selection of our books indexed in the Book Citation Index in Web of Science ${ }^{\mathrm{TM}}$ Core Collection (BKCI)

Interested in publishing with us?

Contact book.department@intechopen.com

Numbers displayed above are based on latest data collected.

For more information visit www.intechopen.com

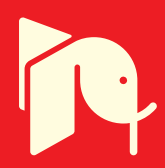





\section{Meet the editor}

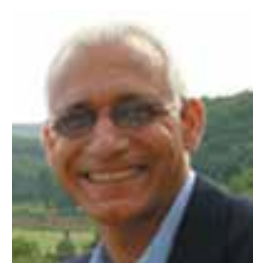

Dr. Zaid Afawi received his MD with honors from the University of Napoli Federico II, Italy and MPH from the Ben-Gurion University of the Negev. During his PhD at Tel-Aviv University he worked with an Australian group and together they discovered new genes and new mutations. He is currently a coordinator of research on genetics of epilepsy in Tel-Aviv Medical Center and Tel-Aviv Medical School University; and a member of staff in Ben-Gurion University of the Negev in Israel. 



\section{Contents}

\section{Preface XI}

Part 1 Mechanisms Underlying Epileptic Seizures 1

Chapter 1 A Functional Role for Microglia in Epilepsy 3 Martine M. Mirrione and Stella E. Tsirka

Chapter 2 The Blood-Brain Barrier in Epilepsy 23 Björn Bauer, Juli Schlichtiger, Anton Pekcec and Anika M.S. Hartz

Chapter 3 New Tools for Understanding Epilepsy $\mathbf{5 5}$

Fatima Shad Kaneez and Faisal Khan

Chapter 4 Epilepsy: Selenium and Aging $\mathbf{7 5}$

Caroline Rocourt, Ying Yu

and Wen-Hsing Cheng

Part 2 Molecular Genetics of Epilepsy 93

Chapter 5 The Molecular Genetics of the Benign Epilepsies of Infancy 95 Sarah E. Heron and John C. Mulley

Part 3 Animal Models 113

Chapter 6 Audiogenic Seizures - Biological Phenomenon and Experimental Model of Human Epilepsies 115 Inga I. Poletaeva, Irina B. Fedotova, Natalia M. Sourina and Zoya A. Kostina

Part 4 Ion and Channels 149

\section{Chapter 7 Ionic Imbalance $\mathbf{1 5 1}$}

John Robert Cressman, Christine Drown and Monica Gertz 


\section{Part 5 New Treatments 173}

Chapter 8 Antiepileptic Medicinal Plants used in Traditional Medicine to Treat Epilepsy 175

E. Ngo Bum, G.S. Taiwe, F.C.O. Moto, G.T. Ngoupaye, R.R.N. Vougat, V.D. Sakoue, C. Gwa, E.R. Ayissi, C. Dong,

A. Rakotonirina and S.V. Rakotonirina

\section{Chapter 9 The Potential Role of ATP-sensitive Potassium} Channels in Treating Epileptic Disorders 193 Chin-Wei Huang 


\section{Preface}

This book on Epilepsy was conceived and produced as a source of information on wide range of issues in epilepsy. We hope that it will help health care providers in daily practices and increase their understanding on diagnosis and treatment of epilepsies.

The book was designed as an update for neuroscientists who are interested in epilepsy, primary care physicians and students in health care professions.

This epilepsy book is the result of a collaborative effort of investigators who have used a wide range of experimental preparations and recording techniques. Authors from a variety of backgrounds have contributed significantly with papers from their respective fields. I believe they have provided a comprehensive description of key issues and important developments within each filed of research.

The studies included in this book are drawn from several disciplines of modern neuroscience and include various Chapters distributed in sections on Mechanisms Underlying Epileptic Seizures, Molecular Genetics of Epilepsy, Animal Models, Ion and Channels and New Treatments

Zaid Afawi

Tel-Aviv Medical Center

Tel-Aviv,

Israel 



\section{Part 1}

Mechanisms Underlying

Epileptic Seizures 



\title{
A Functional Role for Microglia in Epilepsy
}

\author{
Martine M. Mirrione ${ }^{1,2,3}$ and Stella E. Tsirka ${ }^{1}$ \\ ${ }_{1}^{1}$ Department of Pharmacological Sciences, Molecular and Cellular Pharmacology, Stony \\ Brook University, Stony Brook, New York \\ ${ }^{2}$ Medical Department, Brookhaven National Laboratory, Upton, New York \\ ${ }^{3}$ Cold Spring Harbor Laboratory, Cold Spring Harbor, New York
}

USA

\section{Introduction}

Microglia are the immune competent cells of the CNS and comprise the major mechanism of self-defense against brain injury, infections and disease. Activation of microglia occur as a response to these insults, and both neurotoxic and neuroprotective factors can be released (Streit et al., 1999, Streit, 2002, Schwartz, 2003, Schwartz et al., 2006). There is a great deal of evidence suggesting that microglia have a role in neurodegenerative diseases, either to promote the pathology, or to counter it. However in the case of epilepsy, specific questions still remain including how, why, and when microglia are activated. In this first section, we will provide a general introduction to the role of activated microglia in the central nervous system (CNS). In subsequent sections, we will specifically discuss evidence of a functional role for microglia in epilepsy.

In the normal brain, the majority of microglia are in the resting, or quiescent ramified state. This shape with their long processes, allows them to quickly assess and respond to CNS injury or pathogens. During excitotoxic insult or inflammation, microglia become activated. This activation is manifested morphologically by retraction of their processes and changing to a rounded amoeboid morphology. Microglia also become proliferative and migratory. Their electrophysiological characteristics are altered and the expression of potassium, proton, sodium, calcium and chloride currents changes (Eder, 1998, Ducharme et al., 2007, Averaimo et al., 2010, Skaper, 2011). Once activated, the primary function of microglia is to return injured tissue to homeostasis (Streit and Xue, 2009), but this is a double edged sword, as their presence can be both 'good' and 'bad' for neurons. These effects are complex and overlapping, and not necessarily mutually exclusive.

In an effort to protect surviving cells during pathological conditions, microglia have been shown to 'execute' damaged or dying neurons injured from excitotoxicity in order to protect nearby cells from lytic release of toxic intracellular contents. Thus, microglia will facilitate local tissue repair by phagocytosing these cells and cell debris. This occurs through initial mobilization of cells near the site of injury and recruitment of distant microglia into the damaged area, which release proinflammatory mediators. At appropriately minimal and transient microglial activation, this process is ultimately neuroprotective (Vilhardt, 2005). However unregulated hyperactivation and release of toxic factors, such as nitric oxide (NO), 
leading to over production of excess peroxynitrite, and reactive oxygen and nitrogen species (ROS, RNS), may result in an unmanageable level of oxidative stress causing degeneration in nearby 'bystander' cells. Oxidative stress can cause further neuroinflammation as well by recruitment of peripheral immune cells into the damaged brain. This could occur through a compromised blood-brain-barrier (BBB) as can be the case in stroke, Alzheimer's disease, amyotropic lateral sclerosis, and epilepsy (del Zoppo et al., 2000, Mhatre et al., 2004, van Vliet et al., 2007).

Microglia can also be modulatory by secreting a host of inflammatory mediators upon activation, including cycloxygenase-2 (Cox-2), interleukin-3 (Il-3), interleukin-6 (Il-6), interleukin-1beta (IL-1 $\beta$ ), tumor necrosis factor-alpha (TNF- $\alpha$ ), prostaglandins (PGs), tissue plasminogen activator (tPA), monocyte chemoattractant protein-1 (MCP-1), vascular endothelial growth factor (VEGF), lymph toxin, matrix metalloproteinases (MMPs), and macrophage inflammatory protein-1alpha (MIP-1a). The identity, quantity and duration of release of these factors can vary widely based on the specific injury. Some of these molecules can be toxic in high amounts, and certainly can have a direct impact on neuronal function. For example, we have studied the protease tissue plasminogen activator (tPA), which along with other trophic factors released by microglia, has been demonstrated to be critical in the sprouting of mossy fibers emanating from the dentate gyrus (DG) (Wu et al., 2000, Ferrer, 2002, Zhang et al., 2004) and may potentially facilitate seizure recruitment and the chronic maintenance of convulsions (Sloviter et al., 1996, Buckmaster et al., 2002, Shibley and Smith, 2002, Winokur et al., 2004). Indeed a number of studies including our own, have suggested that tPA from neurons, and potentially also microglia, plays a role in mediating seizure development (Qian et al., 1993, Schmoll et al., 2003, Yepes and Lawrence, 2004, Pawlak et al., 2005, Mirrione et al., 2007).

There is as well, a good side to this double-edged 'microglia' sword. They can also release several neuroprotective factors, such as neurotrophins including brain-derived neurotrophic factor (BDNF) and nerve growth factor (NGF), which can in fact promote neuronal stability (Elkabes et al., 1996, Miwa et al., 1997, Elkabes et al., 1998, Heese et al., 1998, Nakajima et al., 2001a). Maximizing this neuroprotective function can become particularly important in the aging brain as microglia cells enter a phase of senescence, and aged neurons require greater neuroprotection (Streit et al., 2008). Microglia can also positively influence pathological conditions by facilitating the recovery of injured neurons through release of trophic factors as well as extracellular matrix molecules, such as thrombospondin, important for sustaining neuronal function (Chamak et al., 1994). Additionally, application of microglia to the site of spinal cord injury has been shown to improve regenerative neurite outgrowth (Rabchevsky and Streit, 1997).

During CNS injury and glutamate excitotoxicity, microglia can act as both antigen presenting cells (Neumann et al., 1996, O'Keefe et al., 2001), and as cells that can remove harmful materials, including glutamate. Activated microglia express the high affinity glutamate transporter GLT-1, and thus can contribute approximately $10 \%$ of glutamate recycling (Nakajima et al., 2001b, Shaked et al., 2005, Persson et al., 2006), which may become more important under pathological conditions especially if astrocytes are overburdened or impaired. In addition, the cerebellum bergman glia have been shown to be involved in tonic GABA release through the ion channel Best1 (Lee et al., 2010), which may also be expressed and function in microglia in other brain regions (Ducharme et al., 2007). 
While this remains to be explored, tonic GABA release could also be a mechanism for microglia to modulate seizures.

Other ways in which microglia may be beneficial involves the emerging notion of protective autoimmunity (Shaked et al., 2005). During CNS injury such as glutamate toxicity, a proposed mechanism to minimize the destruction would involve building a tolerance to autoimmune self-antigens, and could involve priming or preconditioning of microglia cells (Schwartz et al., 2003). This may be accomplished in several ways, but one experimental way is to expose microglia to lipopolysaccharide (LPS) prior to the injury or pathology. There are a few examples in the literature where preconditioning microglia with LPS modulates seizures including our own, and this will be discussed in more detail in the next section (Sayyah et al., 2003, Akarsu et al., 2006, Arican et al., 2006, Dmowska et al., 2010, Mirrione et al., 2010, Yang et al., 2010a). One mechanism that may contribute to the positive effects attributed to LPS preconditioning is through enhanced neurotrophin release (Nakajima et al., 2001a). While activated rat microglia have indeed been shown to release BNDF, this release is significantly enhanced when they are stimulated with LPS. Furthermore, these activated microglia also released NGF, suggesting that in the context of LPS preconditioning, microglia could be neuroprotective through release of neurotrophins. A careful balance needs to be maintained in dosage and timing of LPS application, as of course it also activates release of potentially toxic factors. Therefore, new strategies to precondition microglia in more refined ways to promote neuroprotective functions, and minimize neurotoxic factor release, may prove to be therapeutically beneficial. In fact one study showed that application of ceramide increased the release of protective factors, but not other potentially dangerous molecules, through specific signaling mechanisms involving protein kinase C (PKC) (Nakajima et al., 2002). As well, interferon-gamma (INF-gamma) administration causes microglia to significantly increase their ability to remove glutamate, also without causing an inflammatory response (Shaked et al., 2005). These strategies should be explored further in the context of seizures and potentially exploited as neuroprotective modulators.

\section{A functional role for microglia in seizures: The 'bad' and the 'good'}

A growing body of evidence suggests that microglia have a functional role in the pathology and symptoms of CNS diseases including ischemia and Alzheimer's disease. In this section, we discuss the evidence linking microglia to seizures and epilepsy. We will focus on examples from the literature suggesting both 'bad' neurotoxic, and 'good' neuroprotective, contributions. However, while it is clear from these data that a functional role for microglia exists, these effects are certainly complex and even contradictory in different literature reports. Are microglia activated concurrent with seizure pathology, and if so what is the timing, duration, location and extent of this activation? There is growing evidence suggesting that yes, extensive microglial activation known as 'microgliosis' occurs in the brain parenchama of patients with recurrent seizure episodes (as well as reactive astrogliosis), and in animal models of epilepsy particularly in the hippocampus (Beach et al., 1995, Drage et al., 2002). Activated microglia are observed in the same hippocampal regions associated with seizure induced neuronal death clinically and in animal models, owing them a reputation for facilitating neuronal malfunction (Beach et al., 1995, Taniwaki et al., 1996, Tooyama et al., 2002, Borges et al., 2003). In patients, microglia activation was found in the sclerotic hippocampus, suggesting that neuronal degeneration continues to occur as a result of 
ongoing seizure activity (Beach et al., 1995). A recent study confirmed the presence both of activated microglial and also immunoreactive leukocytes in tissue resected from patents with intractable medial temporal lobe epilepsy, and in kainic acid (KA) treated mice, either associated with blood vessels or distributed intraparenchymally in the CA1-CA3, hillus, and to a lesser extent, dentate gyrus (Zattoni et al., 2011). Following pilocarpine induced seizures in mice, microgliosis persists for at least 3-31 days in regions of neuronal loss such as the hippocampus and amygdala (Borges et al., 2003, Yang et al., 2010a). However, we have shown that during KA excitotoxicity, microglia activation is associated with tPA release from injured neurons, and thus is a consequence rather than a cause of neurodegeneration, but overall this microglia activation can further exacerbate the injury (Siao et al., 2003). Gliomas have also been associated with epilepsy, and histological examination of patients' tissue following surgical removal has demonstrated that the numbers of activated microglia correlated with the duration of epilepsy, as well as with the frequency of seizures prior to surgical resection (Aronica et al., 2005). Overall, this evidence would suggest that microglia activation is 'bad' for the epileptic brain and is associated with neurodegenerative pathology.

Knowing that microgial activation is associated with seizures and epilepsy is the first step, but what exactly are these cells doing? Given the plethora of molecules microglia release, one can easily imagine numerous potential outcomes. For instance, it has been proposed that microglia activation may contribute to spontaneous recurrent seizures (SRS) by facilitating aberrant migration of newborn neurons in the DG (Yang et al., 2010a). This study showed that LPS injected directly into the DG promoted the development of ectopic hilar basal dendrites in the hippocampus, while addition of minocycline that blocked microglia activation, prevented it. Neurotrophin and cytokine release can contribute to this aberrant granule cell neurogenesis (Scharfman, 2005). The expression of pro-inflammatory transcripts changes after pilocarpine-induced seizures in mice, and includes upregulation of toll-like receptor type 2 (TLR2, a microglia/macrophage marker) and I kappa B alpha (IאB $\alpha$, index of NF-kappa B activation), particularly in areas undergoing neurodenegeration or demyelination (Turrin and Rivest, 2004). Increased microglial activation is also found in adult animals given KA when they have already been exposed to it during adolescence (Somera-Molina et al., 2009). In this model, early-life seizures created an increased susceptibility to seizures later in life ('two-hit seizure' model), when KA is given at postnatal day 5 (P5), and then again at P45. The expression of proinflammatory cytokines IL-1 beta, TNF-alpha, S100B, and the chemokine CCL2 was found to be enhanced, corresponding to increased susceptibility to seizures in the adults.

Recent evidence strengthens a strong implication of cytokine involvement in modulating acute seizures. mRNA expression of TNF-alpha and IL-6 correlated with seizure development in a viral infection model, and knockout mice lacking the receptors for these cytokines showed reduced seizure frequency (Kirkman et al., 2010). These results suggest the innate immune response to viral infection contributes to seizures through cytokine expression, and can potentially occur through modulation of glutamate receptors/transporters on astrocytes. Such modulation can eventually lead to excessive extrasynaptic/extracellular glutamate levels, which could also be detrimental (Choi and Koh, 2008). Another study supports the role of cytokines showing that in brain regions associated with seizure damage from the nerve agent soman, there was an increased production of IL-1alpha and IL-1beta (neurotoxic cytokines by activated microglia) as well as IL-6 (neuroprotective factor released from neurons and astrocytes) at $72 \mathrm{hrs}$, in the 
piriform cortex, hippocampus, and thalamus (Johnson and Kan, 2010). However in this same model, neurotoxic COX-2 expression, which mediates the production of prostaglandins, was found expressed in neurons, but not in microglia or astrocytes (AngoaPerez et al., 2010). In the mouse kindling model, COX-1 from microglia was enhanced in the hippocampus during progression of seizures and the administration of SC-560 (a selective COX-1 inhibitor) or indomethacine (a non-selective COX inhibitor) reduced the progress of seizures (Tanaka et al., 2009). These two studies suggest that in addition to cytokines, cyclooxygenase enzymes derived from neurons and microglia may also play an important role in mediating seizures.

In terms of electrophysiological changes, one study showed that the cortical innate immune response to LPS application actually increased local neuronal excitability. Furthermore, in a subset of animals, this also produced motor seizures (Rodgers et al., 2009). Based on their experimental evidence, the authors suggest that microglial activation may therefore be a potential precursor to seizures, and not a consequence of them. Specifically, they showed that when LPS is applied to the cortex in vivo, the evoked field potential amplitudes were acutely enhanced (as measured within 5min-1hr) and produced focal epileptiform discharges, which were prevented by pre-application of an interlukin-1 receptor antagonist. They suggested that this rapid response may result in increased glutamate and noradrenaline release within $10 \mathrm{~min}$, potentially causing the rapid increases of neuronal excitability (Wang and White, 1999). While this study showed that LPS induced microglial activation produces increases in neuronal excitability, they only measured short time points, and so further experiments are necessary to determine whether this effect is long lasting and can truly facilitate seizure symptoms.

Although the aforementioned data suggest a strict neurotoxic role of microglia, it must be kept in mind however, that this activation is a response to ongoing injury and abnormal circuitry rewiring. Little affirmative evidence is available suggesting that microglia are causing neuronal malfunction directly (with the exception of the acute study by Rogers et. al. described above) especially since this evidence comes from rodent models where chemoconvulsants are typically used to trigger the symptoms. Therefore it is important to establish whether microgliosis is a consequence of recurrent seizure episodes or a direct early contributor to symptoms. This is inherently complex however, based on their multiple functions as described in the first section.

The most obvious way to probe these questions is to pharmacologically activate and inhibit microglial activation before, during, and after experimental seizure induction and monitor differences in acute seizure symptoms and pathology. Another way to study this is to examine seizures in knockout mice missing integral components of the inflammatory response. Zattoni et al (Zattoni et al., 2011) showed that selective pharmacological ablation of peripheral macrophages prior to kainate injection actually increased dentate granule cell degeneration. This result suggested that the F4/80 (microglia/macrophage marker) positive cells, specifically of peripheral origin, appear to be required for long-term survival of granule cells. The same investigators also showed enhanced neurodegeneration in mutant mice lacking B or T cells, also suggesting a strong impact of immune-mediated responses on network excitability. Overall, these findings support the idea that lymphocytes and macrophages infiltrating the epileptic focal area may have a neuroprotective role.

Several other groups including ours have used LPS to activate microglia at different time points prior to seizures. An interesting result that may be counterintuitive given all the studies described above, is that preconditioning microglia before seizures has shown to be 
potentially protective. This is akin to reports that sublethal stress stimuli induce tolerance in ischemia (Marsh et al., 2009). In one study, LPS administration prior to pentylenetetrazole (PTZ)-induced seizures was beneficial by increasing plasma levels of NO and IL-6, which reduced blood brain barrier permeability (Arican et al., 2006). In kindling, LPS was inhibitory but only when it was administered daily for 16 days, as it blunted the acquisition of kindled behavioral seizures (Sayyah et al., 2003). Neuronal protection from cell death in hippocampal CA1, CA3 and DG was observed with LPS preconditioning 72 hours prior to seizure induction, although seizure behavior was not affected at this low dose of LPS (Dmowska et al., 2010). Importantly, one study addressed the time course of LPS delivery prior to seizure induction and demonstrated complex effects (Akarsu et al., 2006). This group showed that LPS given $4 \mathrm{hr}$ before PTZ-induced seizures was pro-epileptic, but when given $18 \mathrm{hr}$ before, it conferred anticonvulsant effects attributable to the expression of COX1 and -2. It is possible that LPS stimulated release of cytokines concurrent with PTZ drives the pro-convulsant effect, but how protection is conferred by delaying seizure induction for hours to days after LPS administration is less obvious. Tolerance to excitatory input, depletion of intracellular stores of proinflammatory mediators, and upregulation of glutamate recycling in anticipation of the next insult, are all plausible explanations which need further study.

Important questions that remain include what are the circumstances that cause microglia activation during seizures specifically, and are microglia strictly necessary for seizure development? We were surprised recently in studying these questions to find that the activation state of microglia in the hippocampus has a direct impact on the sensitivity of acutely induced seizures. In our experiment, we asked whether conditional microglia/macrophage ablation could affect epileptogenesis using genetically modified mice (Mirrione et al., 2010). These mice express the herpes simplex virus thymidine kinase (HSVTK) gene under the CD11b macrophage/microglia promoter (CD11b-HSVTK+/-). As shown in the schematic of Figure 1a, activated microglia are present throughout hippocampal subfields associated with seizures. Ganciclovir (GCV) is administered intrahippocampally through an implanted osmotic mini-pump. Over time, GCV can be taken up by cells in the hippocampus near the infusion site, which extends throughout the ipsilateral hippocampus. Subsequently, LPS and pilocarpine can be administered i.p. during the experiment. Figure $1 \mathrm{~b}$ describes the effect of the HSVTK transgene which is under the control of the $\mathrm{Cd} 11 \mathrm{~b}$ promoter, and thus expressed exclusively in microglia and macrophages. Cd11b is expressed in resting, ramified microglial cells at low baseline levels, which results in some moderate HSVTK expression. However, cells that are activated (by LPS or pilocarpine) begin to upregulate Cd11b, and thus will express more of the HSVTK suicide gene. HSVTK by itself does not harm the cell, similar to GCV, which by itself is harmless. However, cells that contain both HSVTK and GCV will be susceptible to ablation. HSVTK will phosphorylate GCV, which is further phosphorylated by intracellular kinases. This toxic triphosphate then competes with thymine for DNA synthesis, and causes DNA replication failure killing the cell (Heppner et al., 2005). Therefore, one can selectively target microglia/macrophages for ablation as the expression of Cd11b is restricted to these cells, and increased in activated microglia. This drives increased expression of the HSVTK gene, which causes apoptosis. Microglia/machrophage cells that are in S phase of cellular division are particularly susceptible to ablation using this method, however non-dividing cells are also susceptible, albeit at reduced levels, which may be due to interference with mitochondrial DNA synthesis (Herraiz et al., 2003). 

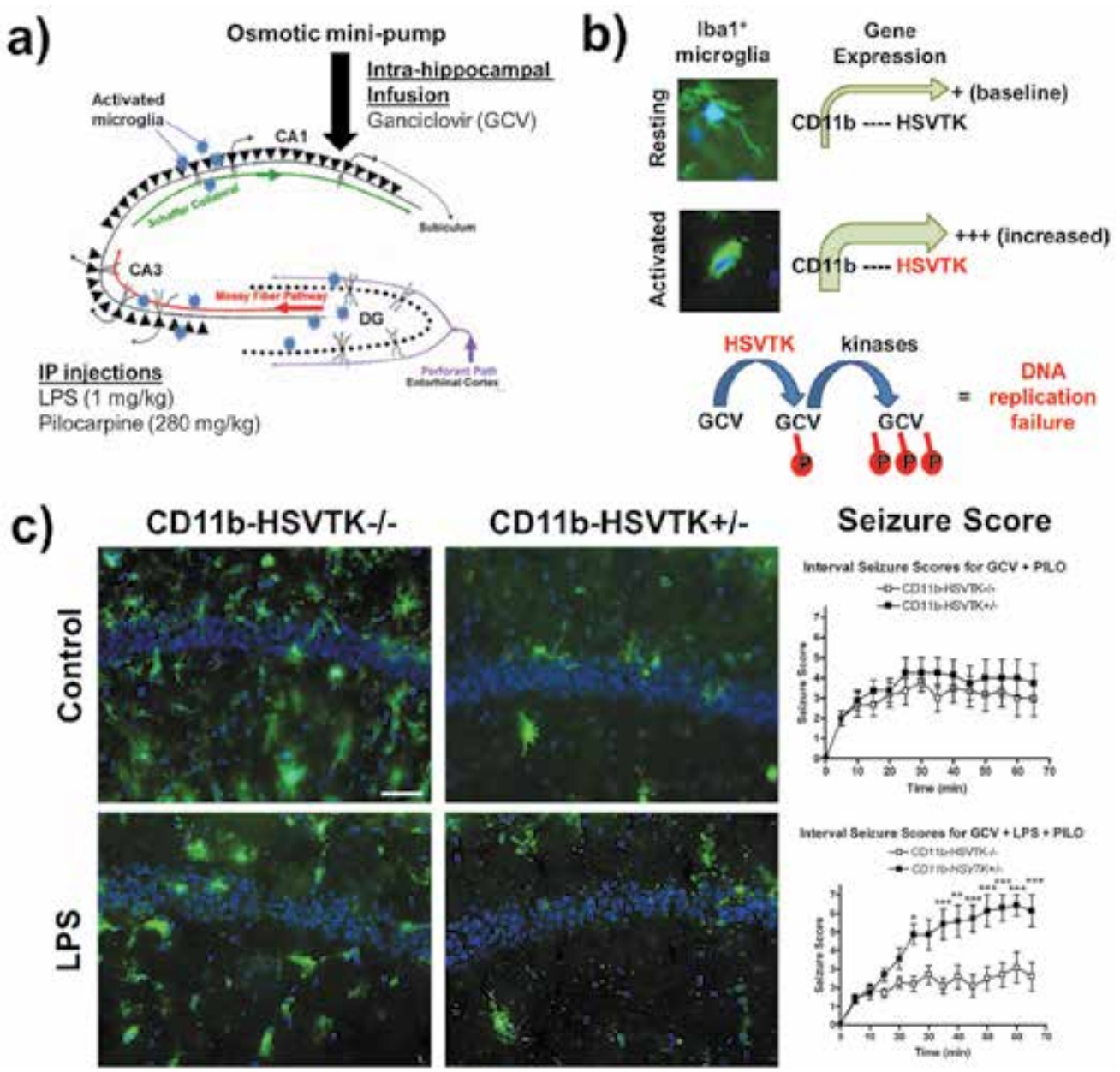

Fig. 1. Microglial ablation and lipopolysaccharide preconditioning modulates pilocarpineinduced seizures in mice. A) Activated microglia are present in hippocampal subfields following LPS and/or pilocarpine induced seizures, Neuronal circuitry in the hippocampus consists of a mainly unidirectional loop of excitatory and inhibitory signals known as the 'trisynaptic circuit', although additional connections between subfields also exist. These neurons are vulnerable to neurodegeneration in seizure models, and DG neurons specifically undergo circuitry rewiring propagating seizure activity. We have shown that that the activation state of microglia in the hippocampus can have a direct impact on the sensitivity of acutely induced seizures. In the experiment, the hippocampus is targeted with intra-hippocampal infusion of ganciclovir (GCV) through an osmotic mini-pump. LPS and pilocarpine are given intraperitoneally (i.p.) and seizure symptoms are recorded. B) Mechanism of cell ablation via GCV and HSVTK. The morphology of resting and activated microglia are shown. The microglia/machrophage specific integrin protein, $\mathrm{Cd} 11 \mathrm{~b}$ (the alpha chain of the Mac-1 integrin), has low expression in resting cells, and is increased in activated cells. The HSVTK suicide gene is placed under the control of the promotor CD11b. When microglia are activated, CD11b expression increases and HSVTK is highly expressed. In a cell which has taken up GCV, and is expressing HSVTK, GCV is phosphorylated by 
HSVTK and endogenous kinases (phosphorylation represented by red circle's labeled ' $\mathrm{P}$ '). This turns GCV into a toxic triphosphate which competes with endogenous thymine for DNA synthesis. Ultimately, DNA replication is disrupted and the cell undergoes apoptosis. C) Data showing CD11b-HSVTK-/- (wild type, left) mice compared to CD11b-HSVTK-/(transgenic mice, right) under control and LPS preconditioning (CA1 subfield, DAPI (blue) and Iba1 (green), scale bar $50 \mu \mathrm{m}$ ) conditions. There were significantly fewer Iba1 positive cells (microglia/macrophages) in the transgenic mice following GCV in both conditions; however seizure symptoms (right panels) only showed a significant divergence, when LPS was administered $24 \mathrm{hr}$ before the pilocarpine injection. In this group, seizure scores were dramatically increased over the first $60 \mathrm{~min}$ following pilocarpine injection. Thus in the absence of LPS activated microglia/macrophages, seizure symptoms were significantly elevated. Additionally, LPS preconditioning in CD11b-HSVTK-/- (wild type) mice also showed a trend toward reducing seizure scores. (Part C, adapted and partially reproduced from (Mirrione et al., 2010)).

Our results showed that unilateral ablation of quiescent (non-activated) microglia from the dorsal hippocampus did not necessarily alter acute seizure sensitivity (Figure 1 c, top panel), but ablation of activated microglia (by LPS given $24 \mathrm{hrs}$ prior to pilocarpine) did in fact significantly enhance seizure symptoms (Figure 1c, bottom panel, and summarized in Table 1). Furthermore, we observed a trend toward reduced seizure activity in LPS preconditioned mice, similarly to what other groups have reported (Sayyah et al., 2003, Akarsu et al., 2006, Arican et al., 2006, Dmowska et al., 2010). These data would suggest that LPS activated microglia were providing some protection to the hippocampus, perhaps by controlling the spread of seizure activity. Additional studies are required to identify the chemical mediators released by microglia that may underlie this protective effect, and whether bilateral microglia/macrophage ablation would be further protective As well, it would be interesting to further evaluate whether neurodegeneration patterns are altered at chronic time points. In regards to how LPS activated microglia upregulate $\mathrm{Cd} 11 \mathrm{~b}$, one group showed this was mediated by nitric oxide (NO) (Roy et al., 2006, Roy et al., 2008). Therefore, LPS may be enhancing inducible nitric-oxide synthase (iNOS) in microglia, which produces NO, upregulating Cd11b, and concurrently the HSVTK gene. This would increase the percentage of cells ablated by GCV over time, potentiating the response to pilocarpine (Table 1). Furthermore, there is evidence as well that ablation of macrophages, and neutrophils which also express CD11b, may certainly play an important role, and shouldn't be discounted as potential mediators (Zattoni et al., 2011). In the future, these transgenic mice can be extremely useful for in depth examination of conditional microglial/macrophage ablation in other brain regions such as the amygdala, cortex, or more specifically targeted subfield regions of the hippocampus.

\begin{tabular}{|c|c|c|c|c|c|c|}
\hline $\begin{array}{l}\text { Allcroglla In iransgenle mlee } \\
\text { (CD11b+HSYTK ti.) }\end{array}$ & $\begin{array}{l}\text { Expres: } \\
\text { CDith }\end{array}$ & on level & $\begin{array}{l}\text { \#In S phase } \\
\text { (dividing) }\end{array}$ & GCY & Cell death & $\begin{array}{c}\text { Response to PILD } \\
\text { (compared to CD11b.HSVTK-i-) }\end{array}$ \\
\hline \multirow{2}{*}{ 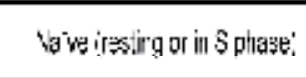 } & \multirow{2}{*}{ - } & \multirow{2}{*}{+} & \multirow{2}{*}{+} & No & 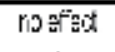 & 1.5 \\
\hline & & & & Yes & + & 1.5. \\
\hline LFS rictivated & +-+ & $H+$ & -++ & $\begin{array}{l}\text { No } \\
\text { Yer: }\end{array}$ & 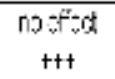 & $\begin{array}{c}1.5 \\
\text { poter:iated }\end{array}$ \\
\hline
\end{tabular}

4.

Table 1. Expression of transgene based on activaction state of microglia. 
In our study, we also explored whether changes in glial function were a component of metabolic changes in these mice following pilocarpine using small animal imaging with 2deoxy-2[(18)F]fluoro-d-glucose (18FDG) and positron emission tomography (PET). Our goal was to find the brain regions involved in the behavioral divergence we observed in CD11bHSVTK+/- mice, compared to wild type mice, using a systems approach. In pilocarpine treated animals of both genotypes, we found increased 18 FDG uptake during seizures in the septum, thalamus, hippocampus, midbrain, and cerebellum, along with decreases in the striatum, which was consistent with our previous findings (Mirrione et al., 2007). In addition, we observed reduced metabolic activation in the ipsilateral hippocampus corresponding to the location of unilateral microglial ablation. While we interpreted this result with caution, we concluded that activated microglia may contribute a small, but potentially important, proportion of the metabolic signal. It is possible then, that changes in glial function could be a component of metabolic abnormalities which are observed in epileptic patients (Lamusuo et al., 2001, Goffin et al., 2008b) and rodent seizure models (Kornblum et al., 2000, Mirrione et al., 2006, Mirrione et al., 2007, Goffin et al., 2009). Therefore, in the next section we will discuss how neuroimaging approaches using specific radiotracers which target microglia, can facilitate our understanding of the pathology underlying epileptogenesis.

\section{Neuroimaging as a potential tool for exploring in vivo function of microglia during epileptogenesis}

We and others have taken advantage of the unique systems level, in vivo neuroimaging modalities to probe questions related to seizure circuitry and malfunction. These techniques have become increasingly utilized both clinically and in basic research to understand epileptogenesis. Metabolic neuroimaging with positron emission tomography (PET) is the most widely used diagnostic, (for review (Mirrione and Tsirka, 2011). A handful of recent studies in animal models have also begun to utilize specific radioligands studying GABA, serotonin, dopamine, and the cannabinoid systems (Werhahn et al., 2006, Goffin et al., 2008a, Liefaard et al., 2009, Liew et al., 2009, Assem-Hilger et al., 2010). There are also a few pilot studies in the literature examining the acetylcholine (Mohamed et al., 2005) and opioid systems (Theodore et al., 1992) in epilepsy patients. While the availability of these radioligands is still somewhat limited in clinical settings, and requires further validation, they may prove to be valuable tools for future research and diagnosis.

PET imaging utilizes a radiotracer, a radiolabeled molecule, that when injected intravenously into the subject will bind specifically to the location of interest. For microglia, the radiotracer should be a ligand which binds to a receptor or transporter expressed specifically on activated microglia. The ligand needs to be chosen carefully, as it would need to exhibit the necessary pharmacokinetic properties to make it a good radiotracer. Specifically, it should effectively enter the central nervous system through the blood stream by crossing the blood brain barrier (lipophilicity is expressed as $\log \mathrm{D}$, or $\log \mathrm{P}$ ), and it should bind to activated microglia (known as the affinity, or dissociation constant Kd) with enough strength to be injected in very low amounts, but still located by the PET camera. Other helpful properties of these radiotracers (radioligands) are that they have low nonspecific binding (that they do not bind randomly to other molecules), and are they are not converted into 'active' metabolites, which can bind to some other unwanted target in the brain and perturb the analysis. 
The first major radioligand that has been used to study activated microglia is carbon-11 labeled PK11195, a compound that binds to the peripheral benzodiazepine receptor (PBR). The PBR is a mitochondrial protein expressed on both microglia and macrophages. Its expression is increased after brain injury or neuroinflammation. [11C]PK11195 was used in a pilot study comparing 4 normal human subjects, 3 patients with clinically stable hippocampal sclerosis and low seizure frequency, and 2 patients with histologically confirmed Rasmussen's encephalitis (RE) (Banati et al., 1999). This initial study showed that specific binding of the radiotracer the RE patients showed a focal and diffuse increase in binding throughout the seizure-affected hemisphere. Interestingly, the clinically stable hippocampal sclerosis group was similar to normal controls, suggesting that at this stage for these few patients, activated microglia were no longer contributing to the symptoms or pathology. Furthermore, a case of cerebral vasculitis in refractory epilepsy was cured with ${ }^{[11}$ C]PK11195 imaging (Goerres et al., 2001). This adult female patient showed an area of increased radiotracer signal in the left occipital lobe. Biopsy showed that this was positive for activated microglia, vascular and perivascular accumulations of mononuclear cells. The patient was then treated with prednisolone and azathiorprine (immune suppressing drugs) which subsequently reduced her seizures.

Based on these initial studies, a recent case report using [11C]PK11195 of encephalitis facilitated treatment of a 5 year old patient who did not respond to medication (Kumar et al., 2008). Using ${ }^{18}$ FDG was not particularly informative, as this patient showed diffuse glucose hypometabolism without any focal abnormality. As the physicians suspected that underlying brain inflammation was the cause, a [11C]PK11195 scan was conducted which showed an area of increased uptake in the left temporal-occipital cortex. This area was subsequently resected and the patient fully recovered. They concluded that focal areas of neuroinflammation play an important role in refractory seizures associated with encephalitis, and using this imaging approach highlighted the region for surgical removal, and led to a successful recovery. This approach was used again and resulted in the treatment of another female patient with intractable epilepsy due to focal cortical dysplasia (Butler et al., 2011).

One can easily imagine from these case reports how examining the time course of microglia activation over the development and progression of epileptogenesis in patients and animal models can truly shed light on the microglial contribution to pathology. While PK1195 has proven to be a successful indicator of microglia activation in seizures, it has limited uptake in the brain and thus has limited quantitative capacity. Much effort has been put into finding alternative ligands for the PBR which have higher affinity's and better pharmacological properties as radiotracers. The list of these experimental compounds is actually quite long, and the available ligands for studying microglia were recently reviewed (Leung, 2004-2010, Schweitzer et al., 2010). One potential tracer [11C]DAA1106 has been shown to have a higher affinity to the PBR compared to PK1195 and may be more suitable for measuring subtle changes in microglia activation during disease (Maeda et al., 2004). Several other tracers have similar enhanced potential including [125I]DPA-713 (a ligand for SPECT imaging, which also has a slightly higher affinity) (Wang et al., 2009), and [11C]CLINME (Boutin et al., 2007) which has lower non-specific uptake.

Thus far, the most popular target to image microglia remains as the PBR, however other targets for activated microglia should also be explored. New tracers may be significantly useful for examining the impact and progression of microgliosis during different stages of 
epileptogenesis, first in animal models and eventually in patients. We urge the continued development of such radiotracers specifically for this purpose.

\section{Optogenetics as a potential tool for exploring in vivo function of microglia during epileptogenesis}

Optogenetics is an exponentially growing field in neuroscience where light responsive ion channels called opsins are genetically introduced into neurons and astrocytes (Deisseroth, 2011). Thus far, no experimental evidence is available that suggests that microglia could also be targeted with opsins, however this is certainly plausible. Channel rhodopsin-2 can activate neurons with blue light by allowing influx of sodium, but can also allow for influx of calcium, which has been exploited for activating astrocytes (Gradinaru et al., 2009, Figueiredo et al., 2011). Astrocytes in fact, could also be explored in the context of seizures using these methods. Microglia are unique however, in that once activated they express an inward chloride conductance (Averaimo et al., 2010). This could also theoretically be mimicked with expression of halorhodopsin, which when activated with yellow light, allows passage of chloride ions. These swelling-activated chloride channels I (Clswell) are naturally expressed on microglia (Ducharme et al., 2007), and contribute to their phagocytic capacity (Furtner et al., 2007), and to the production of nitric oxide (Kjaer et al., 2009). Similar to experiments on astrocytes, transgenic mouse lines can be created where these channels are expressed under microglia/macrophage specific promoters. Cre-dependent viruses could also be used to target microglia specifically. Implanted optical fibers could then deliver light stimulation to specific brain regions in vivo under various experimental conditions.

This method may be advantageous over pharmacological approaches, as these channels respond very quickly to light on and off by opening and closing, thus allowing for a variety of experimental manipulations in vivo, in real time. Potentially this could allow for fast activation and deactivation of microglia. However, the real kinetics that returns the cells to a resting state once activated is of course very different in glia than neurons. This could potentially be a limitation of this approach, since once microglia are activated, it takes them time to naturally return to a resting state. Despite this caveat, optogenetics may provide an interesting basic research avenue in the future for studying channel properties of microglia.

\section{Microglia as a therapeutic target for epilepsy}

Can microglia be therapeutically targeted to benefit epilepsy patients? While this question has yet to be systematically explored, a few case studies and basic research experiments may suggest a tentative yes. However, given the complexity of microglia-neuronal interactions, a systematic analysis of activated microglia function at various stages during epileptogenesis is necessary.

There are several drugs used experimentally in animal models to modulate the activation state of microglia, which are effective as anti-inflammatory agents. One recent example showed that erythropoietin administered to rats reduced a rise in inflammatory genes, edema, and the numbers of activated microglia after febrile seizures, thereby reducing the risk of subsequent spontaneous seizures (Jung et al., 2011). Another report showed that levetiracetam reduced the harmful spread of excitation during seizures by restoring gap junctions in astrocytes co-cultured with microglia (Stienen et al., 2011). The investigators 
found that TGFbeta1 expression was increased, which normalized the impaired astrocyte membrane resting potentials via modification of inward and outward rectifier currents. TGFbeta1 has also been shown to be a potent anti-inflammatory and neuroprotective agent by either preventing the classical activation of microglia, or by enhancing the presence of alternatively activated microglia, and reducing oxidative stress (Qian et al., 2008).

Inflammatory blockade has also been accomplished with celecoxib, a selective cyclooxygenase-2 inhibitor (Jung et al., 2006). When given during the latent period, it prevented neuronal death and microglia activation in the hilus and CA1. It also inhibited ectopic granule cell formation in the hilus and new glia infiltrating the CA1. Interestingly, COX-2 was shown to be neuroprotective as SC-58236, another selective COX-2 inhibitor, reversed the anti-seizure activity of LPS given $18 \mathrm{hr}$ before PTZ (Akarsu et al., 2006). In the same study, a COX-1 selective inhibitor, valeryl salicylate, facilitated PTZ-induced seizures. However, another group showed that in the mouse kindling model, the administration of SC-560 (a selective COX-1 inhibitor) or indomethacine (a non-selective COX inhibitor) actually reduced the progress of seizures (Tanaka et al., 2009).

Carbamazepine was also anti-inflammatory as it inhibited the production of NO and prostaglandin E2 production (Matoth et al., 2000). Resveratrol, a naturally occurring nonflavonoid polyphenol found in grapes, has been shown to inhibit microglia activation, proinflammatory factor release, and NADPH oxidase which protected neurons (Zhang et al., 2010a, Zhang et al., 2010b). Ghrelin attenuates KA induced cell death in the hippocampus of mice by preventing the activation of microglia and astrocytes and their expression of cytokines TNF-alpha, IL-1b, and COX-2 (Lee et al., 2010a). Minozac, another small molecule that reduces proinflammatory cytokine production, was given following early life seizure induction. Minozac attenuated the enhanced microglial responses that increased vulnerability to seizures in adulthood (Somera-Molina et al., 2009). Similarly, naloxone is also anti-inflammatory, reducing IL-1beta synthesis and microglial activation following early life seizure induction. It also lowered the vulnerability of immature brains to a second seizure episode in adulthood (Yang et al., 2010b).

In terms of cytokines and inflammatory mediators, IL- 1 beta has been shown to be proconvulsant, and IL-1 receptor antagonism (via IL-1Ra) has proven to be a powerful inhibitor of seizures (Vezzani et al., 1999, Vezzani et al., 2000). A recent study reported findings that supported this notion, as IL-1 receptor antagonism blocked the effects of LPS induced enhancement of evoked field potential amplitudes and focal epileptiform discharges in the first minutes to hour of exposure (Rodgers et al., 2009). However, in this case, further study at longer time points should be evaluated to determine if this effect is lasting, or whether it changes, as we and others have shown is the case for LPS on seizure symptoms. Other inflammatory mediators, interleukin-18 (IL-18) and interferon-gamma (INF-gamma) have also proven to be protective against neuronal damage following status epilepticus (SE) (Ryu et al., 2010). Valproic acid, a standard clinical therapy for seizures, was shown to inhibit the production of NF-kappaB, which blocked the release of TNF-alpha and IL-6 by microglia (Ichiyama et al., 2000). It has also been shown to cause microglial apoptosis through the p38 MAPK and mitochondrial apoptosis pathways (Xie et al., 2010).

A recent review highlights the potential for targeting ion channels on microglia to modulate microglial activity (Skaper, 2011). For example, chloride intracellular channel 1 translocation to the activated plasma membrane generates ROS which mediates amyloid beta-peptide 
clearance (Milton et al., 2008, Paradisi et al., 2008). Also, inhibiting the $\mathrm{Ca}^{2+} /$ calmodulineactivated $\mathrm{K}^{+}$channel expressed on microglia actually reduced LPS induced toxicity (Li et al., 2008).

Several studies have been published suggesting positive regulation of microglia can also have beneficial effects for seizure therapy. An intriguing concept is to activate or 'program' microglia with certain drugs to harness their neuroprotective capabilities. Ceramide is one such compound, that when applied in culture, increased the release of protective BDNF, but not other dangerous molecules, TNF-alpha, IL-1beta, or NO (Nakajima et al., 2002). Interferon-gamma (INF-gamma) has been shown to drive microglia to significantly increase their ability to remove glutamate, without causing an inflammatory response (Shaked et al., 2005). Also, insulin-like growth factor-1 (IGF-1) from microglia and astrocytes was shown to promote hippocampal CA1 neuronal survival following injury to dentate granule cells (Wine et al., 2009).

While there is overwhelming evidence that microglia should be inhibited as a means to treat epilepsy, it is important to keep in mind the context of this experimental evidence as it mostly originates from chemoconvulsant models and in vitro measurements. In-vivo situations need to be further explored to fully validate these hypotheses and potential therapeutic approaches. Overall however, the strategies outlined in this section should be assessed in the context of seizures. Of particular interest clinically, is the concept of pharmacologically harnessing the neuroprotective qualities of microglia, while inhibiting their neurotoxic properties.

\section{Summary}

Overall, a growing body of evidence suggests that microglia have a functional role in the pathology and symptoms of epilepsy. Future studies should focus on elucidating the time course of microglial activation during seizure progression, as well as specific factors released at these various times. Furthermore, novel approaches such as in vivo neuroimaging and optogenetics will provide additional avenues to examine these questions. Fully exploiting the therapeutic benefit of modulating microglia for patients with epilepsy has great potential.

\section{Acknowledgements}

This work was supported by the National Institutes of Health (NIH) with funding to S.E.T. (R01NS042168). The authors are grateful to Frank L. Heppner and Adriano Aguzzi for collaboration and sharing the CD11b-HSVTK transgenic mice. We would also like to thank Dr Fritz Henn for support to M.M.M while writing this manuscript.

\section{References}

Akarsu ES, Ozdayi S, Algan E, Ulupinar F (2006) The neuronal excitability time-dependently changes after lipopolysaccharide administration in mice: possible role of cyclooxygenase-2 induction. Epilepsy Res 71:181-187.

Angoa-Perez M, Kreipke CW, Thomas DM, Van Shura KE, Lyman M, McDonough JH, Kuhn DM (2010) Soman increases neuronal COX-2 levels: possible link between seizures and protracted neuronal damage. Neurotoxicology 31:738-746. 
Arican N, Kaya M, Kalayci R, Uzun H, Ahishali B, Bilgic B, Elmas I, Kucuk M, Gurses C, Uzun M (2006) Effects of lipopolysaccharide on blood-brain barrier permeability during pentylenetetrazole-induced epileptic seizures in rats. Life Sci 79:1-7.

Aronica E, Gorter JA, Redeker S, Ramkema M, Spliet WG, van Rijen PC, Leenstra S, Troost D (2005) Distribution, characterization and clinical significance of microglia in glioneuronal tumours from patients with chronic intractable epilepsy. Neuropathol Appl Neurobiol 31:280-291.

Assem-Hilger E, Lanzenberger R, Savli M, Wadsak W, Mitterhauser M, Mien LK, Stogmann E, Baumgartner C, Kletter K, Asenbaum S (2010) Central serotonin 1A receptor binding in temporal lobe epilepsy: A [carbonyl-(11)C]WAY-100635 PET study. Epilepsy Behav.

Averaimo S, Milton RH, Duchen MR, Mazzanti M (2010) Chloride intracellular channel 1 (CLIC1): Sensor and effector during oxidative stress. FEBS Lett 584:2076-2084.

Banati RB, Goerres GW, Myers R, Gunn RN, Turkheimer FE, Kreutzberg GW, Brooks DJ, Jones T, Duncan JS (1999) [11C](R)-PK11195 positron emission tomography imaging of activated microglia in vivo in Rasmussen's encephalitis. Neurology 53:2199-2203.

Beach TG, Woodhurst WB, MacDonald DB, Jones MW (1995) Reactive microglia in hippocampal sclerosis associated with human temporal lobe epilepsy. Neurosci Lett 191:27-30.

Borges K, Gearing M, McDermott DL, Smith AB, Almonte AG, Wainer BH, Dingledine R (2003) Neuronal and glial pathological changes during epileptogenesis in the mouse pilocarpine model. Experimental Neurology 182:21-34.

Boutin H, Chauveau F, Thominiaux C, Kuhnast B, Gregoire MC, Jan S, Trebossen R, Dolle F, Tavitian B, Mattner F, Katsifis A (2007) In vivo imaging of brain lesions with [(11)C]CLINME, a new PET radioligand of peripheral benzodiazepine receptors. Glia 55:1459-1468.

Buckmaster PS, Zhang GF, Yamawaki R (2002) Axon Sprouting in a Model of Temporal Lobe Epilepsy Creates a Predominantly Excitatory Feedback Circuit. J Neurosci 22:6650-6658.

Butler T, Ichise M, Teich AF, Gerard E, Osborne J, French J, Devinsky O, Kuzniecky R, Gilliam F, Pervez F, Provenzano F, Goldsmith S, Vallabhajosula S, Stern E, Silbersweig D (2011) Imaging Inflammation in a Patient with Epilepsy Due to Focal Cortical Dysplasia. J Neuroimaging.

Chamak B, Morandi V, Mallat M (1994) Brain macrophages stimulate neurite growth and regeneration by secreting thrombospondin. J Neurosci Res 38:221-233.

Choi J, Koh S (2008) Role of brain inflammation in epileptogenesis. Yonsei Med J 49:1-18.

Deisseroth K (2011) Optogenetics. Nat Methods 8:26-29.

del Zoppo G, Ginis I, Hallenbeck JM, Iadecola C, Wang X, Feuerstein GZ (2000) Inflammation and stroke: putative role for cytokines, adhesion molecules and iNOS in brain response to ischemia. Brain Pathol 10:95-112.

Dmowska M, Cybulska R, Schoenborn R, Piersiak T, Jaworska-Adamu J, Gawron A (2010) Behavioural and histological effects of preconditioning with lipopolysaccharide in epileptic rats. Neurochem Res 35:262-272.

Drage MG, Holmes GL, Seyfried TN (2002) Hippocampal neurons and glia in epileptic EL mice. J Neurocytol 31:681-692. 
Ducharme G, Newell EW, Pinto C, Schlichter LC (2007) Small-conductance Cl- channels contribute to volume regulation and phagocytosis in microglia. Eur J Neurosci 26:2119-2130.

Eder C (1998) Ion channels in microglia (brain macrophages). Am J Physiol 275:C327-342.

Elkabes S, DiCicco-Bloom EM, Black IB (1996) Brain microglia/macrophages express neurotrophins that selectively regulate microglial proliferation and function. J Neurosci 16:2508-2521.

Elkabes S, Peng L, Black IB (1998) Lipopolysaccharide differentially regulates microglial trk receptor and neurotrophin expression. J Neurosci Res 54:117-122.

Ferrer I (2002) [Cell signaling in the epileptic hippocampus]. Rev Neurol 34:544-550.

Figueiredo M, Lane S, Tang F, Liu BH, Hewinson J, Marina N, Kasymov V, Souslova EA, Chudakov DM, Gourine AV, Teschemacher AG, Kasparov S (2011) Optogenetic experimentation on astrocytes. Exp Physiol 96:40-50.

Furtner T, Zierler S, Kerschbaum HH (2007) Blockade of chloride channels suppresses engulfment of microspheres in the microglial cell line, BV-2. Brain Res 1184:1-9.

Goerres GW, Revesz T, Duncan J, Banati RB (2001) Imaging cerebral vasculitis in refractory epilepsy using [(11)C](R)-PK11195 positron emission tomography. AJR Am J Roentgenol 176:1016-1018.

Goffin K, Bormans G, Casteels C, Bosier B, Lambert DM, Grachev ID, Van Paesschen W, Van Laere K (2008a) An in vivo [18F]MK-9470 microPET study of type 1 cannabinoid receptor binding in Wistar rats after chronic administration of valproate and levetiracetam. Neuropharmacology 54:1103-1106.

Goffin K, Dedeurwaerdere S, Van Laere K, Van Paesschen W (2008b) Neuronuclear assessment of patients with epilepsy. Semin Nucl Med 38:227-239.

Goffin K, Van Paesschen W, Dupont P, Van Laere K (2009) Longitudinal microPET imaging of brain glucose metabolism in rat lithium-pilocarpine model of epilepsy. Exp Neurol 217:205-209.

Gradinaru V, Mogri M, Thompson KR, Henderson JM, Deisseroth K (2009) Optical deconstruction of parkinsonian neural circuitry. Science 324:354-359.

Heese K, Fiebich BL, Bauer J, Otten U (1998) NF-kappaB modulates lipopolysaccharideinduced microglial nerve growth factor expression. Glia 22:401-407.

Heppner FL, Greter M, Marino D, Falsig J, Raivich G, Hovelmeyer N, Waisman A, Rulicke T, Prinz M, Priller J, Becher B, Aguzzi A (2005) Experimental autoimmune encephalomyelitis repressed by microglial paralysis. Nat Med 11:146-152.

Herraiz M, Beraza N, Solano A, Sangro B, Montoya J, Qian C, Prieto J, Bustos M (2003) Liver failure caused by herpes simplex virus thymidine kinase plus ganciclovir therapy is associated with mitochondrial dysfunction and mitochondrial DNA depletion. Hum Gene Ther 14:463-472.

Ichiyama T, Okada K, Lipton JM, Matsubara T, Hayashi T, Furukawa S (2000) Sodium valproate inhibits production of TNF-alpha and IL-6 and activation of NF-kappaB. Brain Res 857:246-251.

Johnson EA, Kan RK (2010) The acute phase response and soman-induced status epilepticus: temporal, regional and cellular changes in rat brain cytokine concentrations. J Neuroinflammation 7:40.

Jung KH, Chu K, Lee ST, Kim J, Sinn DI, Kim JM, Park DK, Lee JJ, Kim SU, Kim M, Lee SK, Roh JK (2006) Cyclooxygenase-2 inhibitor, celecoxib, inhibits the altered 
hippocampal neurogenesis with attenuation of spontaneous recurrent seizures following pilocarpine-induced status epilepticus. Neurobiol Dis 23:237-246.

Jung KH, Chu K, Lee ST, Park KI, Kim JH, Kang KM, Kim S, Jeon D, Kim M, Lee SK, Roh JK (2011) Molecular alterations underlying epileptogenesis after prolonged febrile seizure and modulation by erythropoietin. Epilepsia 52:541-550.

Kirkman NJ, Libbey JE, Wilcox KS, White HS, Fujinami RS (2010) Innate but not adaptive immune responses contribute to behavioral seizures following viral infection. Epilepsia 51:454-464.

Kjaer K, Strobaek D, Christophersen P, Ronn LC (2009) Chloride channel blockers inhibit iNOS expression and NO production in IFNgamma-stimulated microglial BV2 cells. Brain Res 1281:15-24.

Kornblum HI, Araujo DM, Annala AJ, Tatsukawa KJ, Phelps ME, Cherry SR (2000) In vivo imaging of neuronal activation and plasticity in the rat brain by high resolution positron emission tomography (microPET). Nat Biotechnol 18:655-660.

Kumar A, Chugani HT, Luat A, Asano E, Sood S (2008) Epilepsy surgery in a case of encephalitis: use of 11C-PK11195 positron emission tomography. Pediatr Neurol 38:439-442.

Lamusuo S, Jutila L, Ylinen A, Kalviainen R, Mervaala E, Haaparanta M, Jaaskelainen S, Partanen K, Vapalahti M, Rinne J (2001) [18F]FDG-PET reveals temporal hypometabolism in patients with temporal lobe epilepsy even when quantitative MRI and histopathological analysis show only mild hippocampal damage. Arch Neurol 58:933-939.

Lee S, Yoon BE, Berglund K, Oh SJ, Park H, Shin HS, Augustine GJ, Lee CJ (2010) Channelmediated tonic GABA release from glia. Science 330:790-796.

Leung K (2004-2010) N-Benzyl-N-methyl-2-[7,8-dihydro-7-(2-[18F]fluoroethyl)-8-oxo-2phenyl-9H- purin-9-yl]acetamide. In: Molecular Imaging and Contrast Agent Database (MICAD) [Internet] Bethesda (MD): National Center for Biotechnology Information (US); 2004-2010.

Li F, Lu J, Wu CY, Kaur C, Sivakumar V, Sun J, Li S, Ling EA (2008) Expression of Kv1.2 in microglia and its putative roles in modulating production of proinflammatory cytokines and reactive oxygen species. J Neurochem 106:2093-2105.

Liefaard LC, Ploeger BA, Molthoff CF, de Jong HW, Dijkstra J, van der Weerd L, Lammertsma AA, Danhof M, Voskuyl RA (2009) Changes in GABAA receptor properties in amygdala kindled animals: in vivo studies using [11C]flumazenil and positron emission tomography. Epilepsia 50:88-98.

Liew CJ, Lim YM, Bonwetsch R, Shamim S, Sato S, Reeves-Tyer P, Herscovitch P, Dustin I, Bagic A, Giovacchini G, Theodore WH (2009) 18F-FCWAY and 18F-FDG PET in MRI-negative temporal lobe epilepsy. Epilepsia 50:234-239.

Maeda J, Suhara T, Zhang MR, Okauchi T, Yasuno F, Ikoma Y, Inaji M, Nagai Y, Takano A, Obayashi S, Suzuki K (2004) Novel peripheral benzodiazepine receptor ligand [11C]DAA1106 for PET: an imaging tool for glial cells in the brain. Synapse 52:283291.

Marsh B, Stevens SL, Packard AE, Gopalan B, Hunter B, Leung PY, Harrington CA, StenzelPoore MP (2009) Systemic lipopolysaccharide protects the brain from ischemic injury by reprogramming the response of the brain to stroke: a critical role for IRF3. J Neurosci 29:9839-9849. 
Matoth I, Pinto F, Sicsic C, Brenner T (2000) Inhibitory effect of carbamazepine on inflammatory mediators produced by stimulated glial cells. Neurosci Res 38:209212.

Mhatre M, Floyd RA, Hensley K (2004) Oxidative stress and neuroinflammation in Alzheimer's disease and amyotrophic lateral sclerosis: common links and potential therapeutic targets. J Alzheimers Dis 6:147-157.

Milton RH, Abeti R, Averaimo S, DeBiasi S, Vitellaro L, Jiang L, Curmi PM, Breit SN, Duchen MR, Mazzanti M (2008) CLIC1 function is required for beta-amyloidinduced generation of reactive oxygen species by microglia. J Neurosci 28:1148811499.

Mirrione M, Tsirka S (2011) Neuroimaging in animal seizure models with 18FDG-PET. Epilepsy Research and Treatment.

Mirrione MM, Konomos DK, Gravanis I, Dewey SL, Aguzzi A, Heppner FL, Tsirka SE (2010) Microglial ablation and lipopolysaccharide preconditioning affects pilocarpineinduced seizures in mice. Neurobiol Dis 39:85-97.

Mirrione MM, Schiffer WK, Fowler JS, Alexoff DL, Dewey SL, Tsirka SE (2007) A novel approach for imaging brain-behavior relationships in mice reveals unexpected metabolic patterns during seizures in the absence of tissue plasminogen activator. Neuroimage 38:34-42.

Mirrione MM, Schiffer WK, Siddiq M, Dewey SL, Tsirka SE (2006) PET imaging of glucose metabolism in a mouse model of temporal lobe epilepsy. Synapse 59:119-121.

Miwa T, Furukawa S, Nakajima K, Furukawa Y, Kohsaka S (1997) Lipopolysaccharide enhances synthesis of brain-derived neurotrophic factor in cultured rat microglia. J Neurosci Res 50:1023-1029.

Mohamed A, Eberl S, Fulham MJ, Kassiou M, Zaman A, Henderson D, Beveridge S, Constable C, Lo SK (2005) Sequential 123I-iododexetimide scans in temporal lobe epilepsy: comparison with neuroimaging scans (MR imaging and 18F-FDG PET imaging). Eur J Nucl Med Mol Imaging 32:180-185.

Nakajima K, Honda S, Tohyama Y, Imai Y, Kohsaka S, Kurihara T (2001a) Neurotrophin secretion from cultured microglia. J Neurosci Res 65:322-331.

Nakajima K, Tohyama Y, Kohsaka S, Kurihara T (2001b) Ability of rat microglia to uptake extracellular glutamate. Neurosci Lett 307:171-174.

Nakajima K, Tohyama Y, Kohsaka S, Kurihara T (2002) Ceramide activates microglia to enhance the production/secretion of brain-derived neurotrophic factor (BDNF) without induction of deleterious factors in vitro. J Neurochem 80:697-705.

Neumann H, Boucraut J, Hahnel C, Misgeld T, Wekerle H (1996) Neuronal control of MHC class II inducibility in rat astrocytes and microglia. Eur J Neurosci 8:2582-2590.

O'Keefe GM, Nguyen VT, Ping Tang LL, Benveniste EN (2001) IFN-gamma regulation of class II transactivator promoter IV in macrophages and microglia: involvement of the suppressors of cytokine signaling-1 protein. J Immunol 166:2260-2269.

Paradisi S, Matteucci A, Fabrizi C, Denti MA, Abeti R, Breit SN, Malchiodi-Albedi F, Mazzanti M (2008) Blockade of chloride intracellular ion channel 1 stimulates Abeta phagocytosis. J Neurosci Res 86:2488-2498.

Pawlak R, Melchor JP, Matys T, Skrzypiec AE, Strickland S (2005) Ethanol-withdrawal seizures are controlled by tissue plasminogen activator via modulation of NR2Bcontaining NMDA receptors. Proc Natl Acad Sci U S A 102:443-448. 
Persson M, Sandberg M, Hansson E, Ronnback L (2006) Microglial glutamate uptake is coupled to glutathione synthesis and glutamate release. Eur J Neurosci 24:10631070.

Qian L, Wei SJ, Zhang D, Hu X, Xu Z, Wilson B, El-Benna J, Hong JS, Flood PM (2008) Potent anti-inflammatory and neuroprotective effects of TGF-beta1 are mediated through the inhibition of ERK and p47phox-Ser345 phosphorylation and translocation in microglia. J Immunol 181:660-668.

Qian Z, Gilbert ME, Colicos MA, Kandel ER, Kuhl D (1993) Tissue-plasminogen activator is induced as an immediate-early gene during seizure, kindling and long-term potentiation. Nature 361:453-457.

Rabchevsky AG, Streit WJ (1997) Grafting of cultured microglial cells into the lesioned spinal cord of adult rats enhances neurite outgrowth. J Neurosci Res 47:34-48.

Rodgers KM, Hutchinson MR, Northcutt A, Maier SF, Watkins LR, Barth DS (2009) The cortical innate immune response increases local neuronal excitability leading to seizures. Brain 132:2478-2486.

Roy A, Fung YK, Liu X, Pahan K (2006) Up-regulation of microglial CD11b expression by nitric oxide. J Biol Chem 281:14971-14980.

Roy A, Jana A, Yatish K, Freidt MB, Fung YK, Martinson JA, Pahan K (2008) Reactive oxygen species up-regulate $\mathrm{CD} 11 \mathrm{~b}$ in microglia via nitric oxide: Implications for neurodegenerative diseases. Free Radic Biol Med 45:686-699.

Sayyah M, Najafabadi IT, Beheshti S, Majzoob S (2003) Lipopolysaccharide retards development of amygdala kindling but does not affect fully-kindled seizures in rats. Epilepsy Res 57:175-180.

Scharfman HE (2005) Seizure-induced neurogenesis and its dependence on growth factors and cytokines. Growth Factors and Epilepsy Hauppague: Nova Sciences.

Schmoll H, Badan I, Grecksch G, Walker L, Kessler C, Popa-Wagner A (2003) Kindling status in sprague-dawley rats induced by pentylenetetrazole: involvement of a critical development period. Am J Pathol 162:1027-1034.

Schwartz M (2003) Macrophages and microglia in central nervous system injury: are they helpful or harmful? J Cereb Blood Flow Metab 23:385-394.

Schwartz M, Butovsky O, Bruck W, Hanisch UK (2006) Microglial phenotype: is the commitment reversible? Trends Neurosci 29:68-74.

Schwartz M, Shaked I, Fisher J, Mizrahi T, Schori H (2003) Protective autoimmunity against the enemy within: fighting glutamate toxicity. Trends Neurosci 26:297-302.

Schweitzer PJ, Fallon BA, Mann JJ, Kumar JS (2010) PET tracers for the peripheral benzodiazepine receptor and uses thereof. Drug Discov Today 15:933-942.

Shaked I, Tchoresh D, Gersner R, Meiri G, Mordechai S, Xiao X, Hart RP, Schwartz M (2005) Protective autoimmunity: interferon-gamma enables microglia to remove glutamate without evoking inflammatory mediators. J Neurochem 92:997-1009.

Shibley H, Smith BN (2002) Pilocarpine-induced status epilepticus results in mossy fiber sprouting and spontaneous seizures in C57BL/6 and CD-1 mice. Epilepsy Res 49:109-120.

Siao CJ, Fernandez SR, Tsirka SE (2003) Cell type-specific roles for tissue plasminogen activator released by neurons or microglia after excitotoxic injury. J Neurosci 23:3234-3242. 
Skaper SD (2011) Ion channels on microglia: therapeutic targets for neuroprotection. CNS Neurol Disord Drug Targets 10:44-56.

Sloviter RS, Dean E, Sollas AL, Goodman JH (1996) Apoptosis and necrosis induced in different hippocampal neuron populations by repetitive perforant path stimulation in the rat. J Comp Neurol 366:516-533.

Somera-Molina KC, Nair S, Van Eldik LJ, Watterson DM, Wainwright MS (2009) Enhanced microglial activation and proinflammatory cytokine upregulation are linked to increased susceptibility to seizures and neurologic injury in a 'two-hit' seizure model. Brain Res 1282:162-172.

Stienen MN, Haghikia A, Dambach H, Thone J, Wiemann M, Gold R, Chan A, Dermietzel R, Faustmann PM, Hinkerohe D, Prochnow N (2011) Anti-inflammatory effects of the anticonvulsant drug levetiracetam on electrophysiological properties of astroglia are mediated via TGFbeta1 regulation. Br J Pharmacol 162:491-507.

Streit WJ (2002) Microglia as neuroprotective, immunocompetent cells of the CNS. Glia 40:133-139.

Streit WJ, Miller KR, Lopes KO, Njie E (2008) Microglial degeneration in the aging brain-bad news for neurons? Front Biosci 13:3423-3438.

Streit WJ, Walter SA, Pennell NA (1999) Reactive microgliosis. Prog Neurobiol 57:563-581.

Streit WJ, Xue QS (2009) Life and death of microglia. J Neuroimmune Pharmacol 4:371-379.

Tanaka S, Nakamura T, Sumitani K, Takahashi F, Konishi R, Itano T, Miyamoto O (2009) Stage- and region-specific cyclooxygenase expression and effects of a selective COX-1 inhibitor in the mouse amygdala kindling model. Neurosci Res 65:79-87.

Taniwaki Y, Kato M, Araki T, Kobayashi T (1996) Microglial activation by epileptic activities through the propagation pathway of kainic acid-induced hippocampal seizures in the rat. Neurosci Lett 217:29-32.

Theodore WH, Carson RE, Andreasen P, Zametkin A, Blasberg R, Leiderman DB, Rice K, Newman A, Channing M, Dunn B, et al. (1992) PET imaging of opiate receptor binding in human epilepsy using [18F]cyclofoxy. Epilepsy Res 13:129-139.

Tooyama I, Bellier JP, Park M, Minnasch P, Uemura S, Hisano T, Iwami M, Aimi Y, Yasuhara O, Kimura H (2002) Morphologic study of neuronal death, glial activation, and progenitor cell division in the hippocampus of rat models of epilepsy. Epilepsia 43 Suppl 9:39-43.

Turrin NP, Rivest S (2004) Innate immune reaction in response to seizures: implications for the neuropathology associated with epilepsy. Neurobiol Dis 16:321-334.

van Vliet EA, da Costa Araujo S, Redeker S, van Schaik R, Aronica E, Gorter JA (2007) Blood-brain barrier leakage may lead to progression of temporal lobe epilepsy. Brain 130:521-534.

Vilhardt F (2005) Microglia: phagocyte and glia cell. Int J Biochem Cell Biol 37:17-21.

Wang H, Pullambhatla M, Guilarte TR, Mease RC, Pomper MG (2009) Synthesis of [(125)I]iodoDPA-713: a new probe for imaging inflammation. Biochem Biophys Res Commun 389:80-83.

Wang YS, White TD (1999) The bacterial endotoxin lipopolysaccharide causes rapid inappropriate excitation in rat cortex. J Neurochem 72:652-660.

Werhahn KJ, Landvogt C, Klimpe S, Buchholz HG, Yakushev I, Siessmeier T, Muller-Forell W, Piel M, Rosch F, Glaser M, Schreckenberger M, Bartenstein P (2006) Decreased 
dopamine D2/D3-receptor binding in temporal lobe epilepsy: an [18F]fallypride PET study. Epilepsia 47:1392-1396.

Wine RN, McPherson CA, Harry GJ (2009) IGF-1 and pAKT signaling promote hippocampal CA1 neuronal survival following injury to dentate granule cells. Neurotox Res 16:280-292.

Winokur RS, Kubal T, Liu D, Davis SF, Smith BN (2004) Recurrent excitation in the dentate gyrus of a murine model of temporal lobe epilepsy. Epilepsy Res 58:93-105.

Wu YP, Siao CJ, Lu W, Sung TC, Frohman MA, Milev P, Bugge TH, Degen JL, Levine JM, Margolis RU, Tsirka SE (2000) The tissue plasminogen activator (tPA)/plasmin extracellular proteolytic system regulates seizure-induced hippocampal mossy fiber outgrowth through a proteoglycan substrate. J Cell Biol 148:1295-1304.

Xie N, Wang C, Lin Y, Li H, Chen L, Zhang T, Sun Y, Zhang Y, Yin D, Chi Z (2010) The role of p38 MAPK in valproic acid induced microglia apoptosis. Neurosci Lett 482:5156.

Yang F, Liu ZR, Chen J, Zhang SJ, Quan QY, Huang YG, Jiang W (2010a) Roles of astrocytes and microglia in seizure-induced aberrant neurogenesis in the hippocampus of adult rats. J Neurosci Res 88:519-529.

Yang L, Li F, Ge W, Mi C, Wang R, Sun R (2010b) Protective effects of naloxone in two-hit seizure model. Epilepsia 51:344-353.

Yepes M, Lawrence DA (2004) Neuroserpin: a selective inhibitor of tissue-type plasminogen activator in the central nervous system. Thromb Haemost 91:457-464.

Zattoni M, Mura ML, Deprez F, Schwendener RA, Engelhardt B, Frei K, Fritschy JM (2011) Brain infiltration of leukocytes contributes to the pathophysiology of temporal lobe epilepsy. J Neurosci 31:4037-4050.

Zhang F, Liu J, Shi JS (2010a) Anti-inflammatory activities of resveratrol in the brain: role of resveratrol in microglial activation. Eur J Pharmacol 636:1-7.

Zhang F, Shi JS, Zhou H, Wilson B, Hong JS, Gao HM (2010b) Resveratrol protects dopamine neurons against lipopolysaccharide-induced neurotoxicity through its anti-inflammatory actions. Mol Pharmacol 78:466-477.

Zhang Y, Huang P, Du G, Kanaho Y, Frohman MA, Tsirka SE (2004) Increased expression of two phospholipase D isoforms during experimentally induced hippocampal mossy fiber outgrowth. Glia 46:74-83. 


\title{
The Blood-Brain Barrier in Epilepsy
}

\author{
Björn Bauer1,2, Juli Schlichtiger ${ }^{3}$, Anton Pekcec ${ }^{4}$ and Anika M.S. Hartz ${ }^{1,2}$ \\ 1 University of Minnesota, College of Pharmacy \\ ${ }^{2}$ Brain Barriers Research Center, University of Minnesota, College of Pharmacy \\ ${ }^{3}$ Ludwig-Maximilians University, Department of Nuclear Medicine, Munich \\ ${ }^{4}$ Massachusetts General Hospital, Neuroprotection Research Laboratory \\ $1,2,4$ USA \\ ${ }^{3}$ Germany
}

\section{Introduction}

The blood-brain barrier is altered in epilepsy. This includes altered expression of transporters and metabolic enzymes as well as barrier leakage that have been linked to antiepileptic drug resistance and seizure genesis, respectively. Here we highlight current understanding of these pathological changes. Three critical components of barrier function 1) tight junctions, 2) metabolising enzymes and 3) transporter proteins - are introduced and we describe how they are changed in epilepsy and affected by epilepsy treatment. Recent efforts in blood-brain barrier research to overcome drug-resistant epilepsy are also discussed.

\section{The blood-brain barrier}

The History of Blood-Brain Barrier Discovery. First experiments contributing to the discovery of the blood-brain barrier were performed by Paul Ehrlich in 1885 (Figure 1). Ehrlich observed that water-soluble "vital dyes" injected into the blood of rats did not stain the brain (Ehrlich, 1885). In 1900, Lewandowsky made similar observations and coined the term "blood-brain barrier" ("Bluthirnschranke") to explain this phenomenon (Lewandowsky, 1900). Ehrlich's student, Edwin Goldmann, injected the same dyes Ehrlich had used into the subarachnoid space, and found the opposite: intense staining of the brain but no staining of peripheral tissues (Goldmann, 1909; 1913). Goldmann concluded that a barrier had to exist between the brain and the periphery, thus the concept of a vascular barrier was born. In 1923, Spatz postulated that the brain capillary endothelium had to be the structure responsible for barrier function, which initiated a debate that lasted for decades (Spatz, 1933). It was Reese and Karnovsky, and Brightman and Reese who solved the mystery of the blood-brain barrier in the late 1960s. Using electron microscopy, they discovered that tight junctions connect adjacent capillary endothelial cells and seal the intercellular space (Brightman \& Reese, 1969; Reese \& Karnovsky, 1967). With this, the molecular structure responsible for barrier function was identified and the barrier was localized to the brain capillary endothelium. 


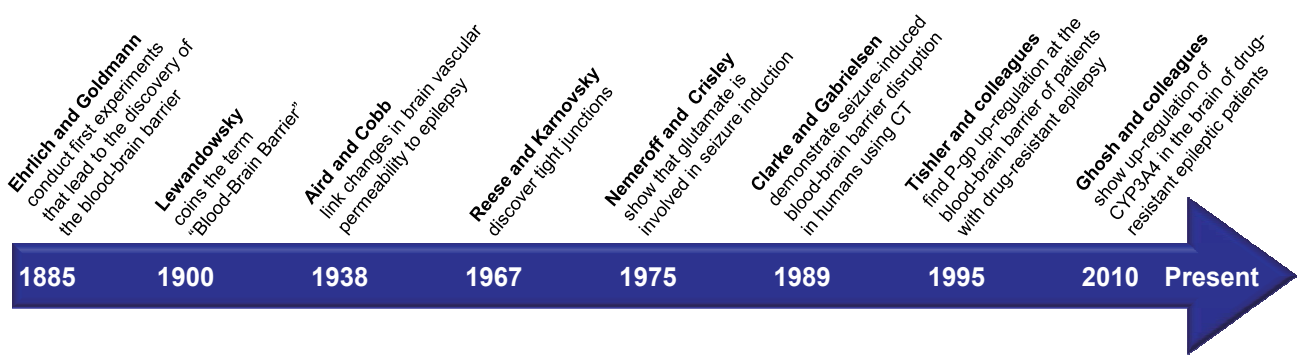

Fig. 1. Evolution of Blood-Brain Barrier Methodology/History

The History of the Blood-Brain Barrier in Epilepsy. In the 1930s, Aird and Cobb discovered that brain uptake of "vital dyes" was increased in epileptic mice. Based on their observation, they suggested that the brain vasculature may be a barrier between the central nervous system (CNS) and the periphery and that altered brain vascular permeability may be a factor contributing to epilepsy (Aird, 1939; Cobb et al., 1938). In the mid 1950s, Bercel used diuretics in patients to increase brain uptake of antiepileptic drugs (AEDs (Bercel, 1955)). Co-administration with diuretics reduced AED doses below toxic levels in ten of ten patients and in seven of these ten patients seizure control was improved (Bercel, 1955). Nemeroff and Crisley made a critical discovery in 1975 when they found that glutamate is involved in seizure induction and increases cerebrovascular permeability in rats (Nemeroff \& Crisley, 1975). Further, blood-brain barrier dysfunction was shown to go along with an increase in blood pressure and cerebral vasodilation during seizures (Bolwig et al., 1977; Petito et al., 1977). In 1989, Clarke and Gabrielsen demonstrated seizure-induced bloodbrain barrier leakage in humans using computed tomography (Clarke \& Gabrielsen, 1989). In 1995, Tishler et al. made the observation that mRNA of MDR1 (ABCB1), the gene encoding the efflux transporter P-glycoprotein (P-gp) is increased at the blood-brain barrier of patients with drug-resistant epilepsy (Tishler et al., 1995). This was a critical finding because P-gp acts as a "gatekeeper" that limits therapeutic drugs from crossing the bloodbrain barrier and from entering the brain (Miller et al., 2008). Research in this field initially focused on P-gp, but other transporters such as multidrug resistance proteins (MRPs) and breast cancer resistance protein (BCRP) are also increased in epilepsy animal models or patients (Awasthi et al., 2005; Dombrowski et al., 2001; Sisodiya et al., 2006; Van Vliet et al., 2005).

Today, the role of some of these transporters in epilepsy is still unclear. It has been discussed that P-gp could be involved in seizure generation (Marchi et al., 2004) and that multiple transporters may act in concert to limit brain uptake of a broad range of AEDs (Lazarowski et al., 2007). Recent studies show that AED-metabolizing enzymes such as cytochromes (CYP) 3A4, 2C8, and glutathione sulfotransferase (GST) $\mu$ and $\Pi$ are also upregulated in the brain of epileptic patients forming a metabolic barrier that contributes to AED resistance (Ghosh et al., 2010; Shang et al., 2008; Ueda et al., 2007).

\subsection{Blood-brain barrier anatomy}

Numbers and Facts. The blood-brain barrier is a network of brain capillaries (microvessels). With a diameter of 3-7 $\mu \mathrm{m}$, brain capillaries are the smallest vessels of the vascular system (Figure 2A) (Rodriguez-Baeza et al., 2003). The microvasculature in the human brain is comprised of about 100 billion capillaries forming a highly branched vascular network 
(Zlokovic \& Apuzzo, 1998). Due to the high capillary density in the brain, capillaries are about $40 \mu \mathrm{m}$ apart from each other, a distance short enough for small molecules to diffuse within 1 second (Rodriguez-Baeza et al., 2003). This ensures that every neuron (about 100 billion in human brain) is in contact with and perfused by its own capillary, which allows efficient nutrient and oxygen supply. Despite the huge number of 100 billion brain capillaries, the total capillary lumen occupies only about $1 \%$ of total brain volume, or about $12-15 \mathrm{ml}$ in an adult human brain of about 1,400 $\mathrm{ml}$ (Pardridge, 2003b). Thus, at any given time, about $8-10 \%$ (about $10 \mathrm{ml}$ ) of total cerebral blood (about $150 \mathrm{ml}$ ) is in the lumen of brain capillaries. Not taking the capillary lumen into account, it is estimated that the brain capillary endothelium occupies only about $0.1 \%$ of total brain volume $(\sim 1-1.5 \mathrm{ml})$ (Pardridge, 2003b). Lastly, the total length of the capillary network is about $600-650 \mathrm{~km}$ in an adult human brain with a total surface area of about $20 \mathrm{~m}^{2}$. This makes the blood-brain barrier the third largest surface area for drug exchange after intestine and lung (Pardridge, 2003a).
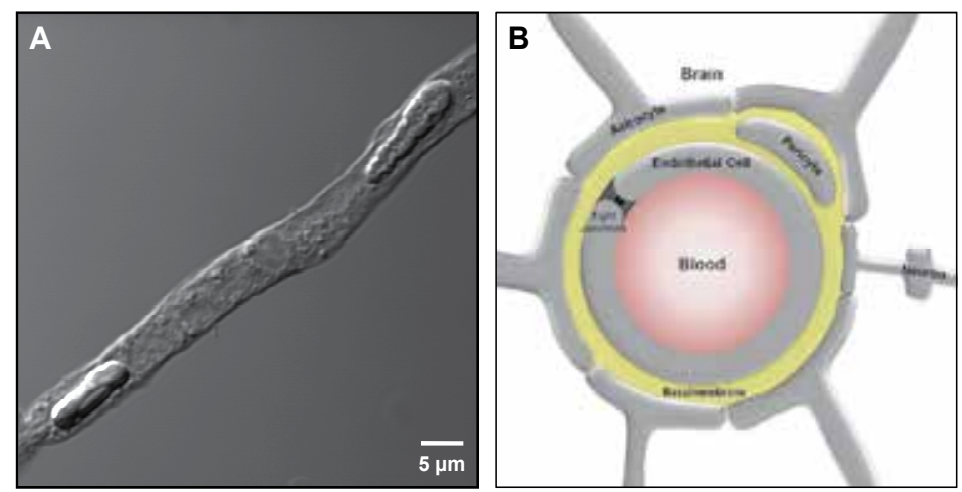

Fig. 2. (A). DIC image of isolated brain capillary. (B) Neurovascular unit.

Morphology and Anatomy. Brain capillaries are the next higher level of organisation from endothelial cells that are the smallest anatomical unit of microvessels. Brain capillary endothelial cells are flat, thin, spindle-shaped, polarized cells. Their apical membrane faces the blood (luminal), and their basolateral membrane faces the brain parenchyma (abluminal; (Betz et al., 1980)). It is through the basement membrane that brain capillary endothelial cells are in contact with pericytes, astrocytes, and neurons (Figure 2B; (Goldstein \& Betz, 1983)). This 4-cell structure is referred to as "Neurovascular Unit" and is responsible for maintaining and regulating blood flow, and for controlling barrier function (Begley, 2004).

One fundamental characteristic of endothelial barrier function is a complex, multi-protein structure called a tight junction, which is unique in the vascular system (Nagy et al., 1984). Brain capillary endothelial cells also lack intercellular clefts and have low pinocytotic activity, which limits solute exchange between blood and brain. Lastly, to meet the large energy demand of ATP-consuming processes like metabolism and active efflux transport, brain capillary endothelial cells possess a large number of mitochondria (Goldstein \& Betz, 1983).

\subsection{Blood-brain barrier physiology}

Blood-brain barrier functions include CNS protection, and regulation and maintenance of CNS homeostasis. Three components determine barrier function: 1. Tight Junctions, 2. 
Transporters and 3. Metabolising Enzymes. The following paragraphs describe these components in more detail.

\section{Tight Junctions}

Tight junctions are cell-cell contacts that seal the intercellular space between adjacent endothelial cells, thereby creating a non-fenestrated endothelium and limiting hydrophilic molecules from paracellular diffusion (Nag, 2003). Tight junctions are multi-protein complexes composed of transmembrane proteins like occludins, claudins, e-cadherins and junctional adhesion molecules as well as adaptor and regulatory proteins (Matter \& Balda, 2003a; Vorbrodt \& Dobrogowska, 2003). Adaptor proteins include zonula occludens proteins, cingulin, catenin and membrane-associated guanylate kinase inverted proteins that connect junctional transmembrane proteins with cytoskeletal actin filaments (Matter \& Balda, 2003b). Regulatory proteins include $G$ proteins, atypical protein kinase $C$ isoforms, and symplektin that are involved in signalling (Matter \& Balda, 2003b; Wolburg \& Lippoldt, 2002). Together, tight junctions guarantee a tight barrier, and thus, protection of the CNS (Kniesel \& Wolburg, 2000). However, under pathological conditions such as epilepsy, tight junctions can be dysfunctional or disrupted, leading to barrier leakage, impaired neuronal function, and brain damage (Huber et al., 2001).

\section{Metabolic Enzymes}

The concept of a "metabolic barrier" is widely accepted but little information is available on metabolising enzymes at the blood-brain barrier. Early studies focused on phase I enzymes in whole brain tissue but later studies differentiated between different brain cell types. Walther et al. showed that CYP P450 enzymes are located in the inner mitochondrial membrane of neurons and glia from rat, guinea pig, rabbit, and pig brain. This is in contrast to liver, where most CYP isoforms are located at the endoplasmic reticulum (Walther et al., 1986). Consistent with this, Ghersi-Egea et al. found CYP P450 protein expression in mitochondria from rat brain tissue (Ghersi-Egea et al., 1987), and demonstrated CYP activity in various brain regions and isolated human microvessels. They found low 1-naphtholUDP-glucuronosyltransferase and NADPH-CYP P450 reductase activity, and high GST and epoxide hydrolase activity (Ghersi-Egea et al., 1993). The same group also found Cyp P450 activity in rat brain microvessels (Ghersi-Egea et al., 1994).

Dauchy et al. used isolated microvessels from resected human brain and found mRNA expression of CYP1A1, 1B1, 2B6, 2C8, 2D6, 2E1, 2J2, 2R1, 2S1, and 2U1, and detected CYP1B1 by Western blotting (Dauchy et al., 2008). Immunohistological studies by Rieder et al. confirmed localisation of CYP1B1 in human brain capillaries (Rieder et al., 2000). In a follow up study, Dauchy et al. showed CYP2U1 and CYP2S1 mRNA expression in the human cerebral microvascular endothelial cell line hCMEC/D3 (Dauchy et al., 2009). CYPs with low mRNA expression included CYP2R1, 2B6, 2E1, 1A1, 2D6, 2C18, 1B1, 2J2, 1A2 and 2C8. Except for CYP2C18, all CYP genes found in hCMEC/D3 cells were also detected in isolated human brain microvessels. A novel CYP P450, Cyp4x1, was identified in 2006 by Al-Aznizy et al. in mouse brain (Al-Anizy et al., 2006). Immunohistochemical staining showed strong Cyp4x1 protein expression in neurons, choroid plexus epithelial cells, and brain microvessel endothelial cells. In 2010, mRNA and protein expression of CYP3A4, the most prominent enzyme involved in xenobiotic metabolism in the liver, was found by Ghosh et al. in human brain endothelial cells (Ghosh et al., 2010).

While most blood-brain barrier enzymes have been detected at the mRNA level, protein expression and activity of only few enzymes have been demonstrated. These include 
gamma-glutamyl transpeptidase (Beuckmann et al., 1995), alkaline phosphatase (Beuckmann et al., 1995), aromatic L-amino acid decarboxylase (Betz et al., 1980; Matter \& Balda, 2003b), the phase I metabolising enzymes CYP1A1 (Filbrandt et al., 2004), CYP1B1 (Filbrandt et al., 2004), CYP3A4 (Ghosh et al., 2010; Ghosh et al., 2011), and Cyp4x1 (AlAnizy et al., 2006), NADPH-CYP P450 reductase (Chat et al., 1998; Ghersi-Egea et al., 1988; Minn et al., 1991; Ravindranath et al., 1990), epoxide hydrolase (Ghersi-Egea et al., 1988; Minn et al., 1991), and the phase II enzymes, 1-naphthol-UDP-glucuronosyltransferase (Ghersi-Egea et al., 1988) and GST $\mu$ (Shang et al., 2008), and GSTп (Bauer et al., 2008; Shang et al., 2008).

The presence of these enzymes in the brain microvasculature indicates the existence of a metabolic barrier. However, more studies are needed to better define the role metabolising enzymes play at the blood-brain barrier under physiological and pathophysiological conditions and whether these enzymes can indeed limit AED delivery to the brain.

\section{Transporters}

The blood-brain barrier is an active, dynamic and selective interface that responds to signals from both the periphery and brain. Key components of barrier function include influx and efflux transporters that are responsible for brain homeostasis, nutrient supply, and protection of the brain from endogenous and exogenous toxins.

Influx transporters that maintain CNS homeostasis and nutrient supply include A-and Nsystem amino acid transporters (Betz et al., 1980; O'kane \& Hawkins, 2003), excitatory amino acid carriers 1, 2, and 3 (O'kane \& Hawkins, 2003; O'kane et al., 1999), alanine/serine/cysteine/threonine (ASCT) transporters for neutral amino acids (Boado et al., 2004; Tayarani et al., 1987), glucose transporters GLUT1 and GLUT3/14 (Pardridge, 1991; Simpson et al., 2007), monocarboxylate transporters MCT1 and MCT8 (Braun et al., 2011; Ito et al., 2011; Simpson et al., 2007), and the equilibrative nucleoside transporter ENT1 (Kitano et al., 2002), as well as $\mathrm{Na}^{+}-\mathrm{K}^{+}$-ATPase (Betz et al., 1980). These transporters belong to the solute carrier (SLC) superfamily. Prominent SLC transporters that have been detected at the bloodbrain barrier also include the organic anion transporter Oat3, organic anion transporting polypeptides Oatp1a4, 1b1, 1c1, 2b1, 14, and organic cation transporters OCT1, OCT2 (Ito et al., 2011; Lin et al., 2010). Of these SLCs, Oat3, Oatps, and Octs are involved in drug transport. However, it is currently not known if these SLC transporters can handle AEDs.

An interesting blood-brain barrier transporter is the large neutral amino acid transporter LAT that transports the amino acids valine, leucine, isoleucine, tryptophan, and tyrosine. LAT1 mediates brain uptake of L-DOPA that is used in Parkinson's disease (Del Amo et al., 2008). LAT1 has also been reported to transport the AEDs gabapentin and pregabalin across the blood-brain barrier into the brain (Del Amo et al., 2008; Liu et al., 2008; Su et al., 1995). Whether LATs are affected in epilepsy is unknown.

In total, 21 transporters have been detected at the protein level in brain capillaries and brain capillary endothelial cells from various species by immunohistochemistry, Western blotting, or quantitative LC/MS/MS (Kamiie et al., 2008; Neuwelt et al., 2011). Seven of these transporters belong to the $\mathrm{ABC}$ (ATP-binding cassette) transporter family and include Pglycoprotein (P-gp, MDR1, $A B C B 1$ ), the multidrug resistance proteins 1, 2, 3, 4, and 5 (MRPs, $A B C C 1-5)$ and breast cancer resistance protein (BCRP, ABCG2). These transporters are ATP-driven and mainly located at the luminal membrane of the brain capillary endothelium (Mrp1 and Mrp4 are also in the abluminal membrane). This "first line of defence" protects the brain from neurotoxicants and limits CNS drugs from entering the brain, and thus, is an obstacle for CNS pharmacotherapy. 
Together, transporters ensure proper CNS nutrient supply and mediate efflux of metabolic wastes from the brain, thus, helping maintain CNS homeostasis. The following section describes the role of transporters, metabolic enzymes, and barrier leakage in epilepsy.

\section{Blood-brain barrier function in epilepsy}

Epilepsy affects more than 60 million people worldwide. The majority of patients respond to treatment with AEDs, but up to $40 \%$ of patients are drug-resistant (Kwan \& Brodie, 2003; Loscher \& Potschka, 2005). Patients with AED resistance suffer from uncontrolled seizures, which elevates their risk of brain damage and mortality (Sperling et al., 1999). These patients experience a low quality of life and, despite advances in pharmacotherapy and neurosurgery, drug-resistant epilepsy remains a major clinical problem (Devinsky, 1999).

Evidence indicates that the blood-brain barrier is altered in patients with epilepsy. Changes in the brain capillary endothelium include upregulation of efflux transporters and metabolic enzymes as well as barrier leakage that have been linked to AED resistance and seizure genesis (Bauer et al., 2008; Ghosh et al., 2010; Marchi et al., 2007). The following section describes the role of transporters, metabolic enzymes, and barrier leakage in epilepsy.

\subsection{Transporters in epilepsy}

One factor underlying AED resistance is, at least in part, seizure-induced over-expression of drug efflux transporters at the blood-brain barrier (Bauer et al., 2008). Some of these transporters, such as P-gp, Mrp2, and BCRP have been implicated with AED resistance. The first evidence for involvement of efflux transporters in epilepsy goes back to studies by Tishler and co-workers in 1995. These researchers observed increased P-gp mRNA in the brain and protein expression in the capillary endothelium of patients with drug-resistant epilepsy (Tishler et al., 1995). The findings by Tishler et al. were confirmed by other groups (Dombrowski et al., 2001; Lazarowski et al., 1999; Sisodiya et al., 2002) and it was suggested that this phenomenon could prevent AEDs from entering the brain and cause AED resistance. However, studies in cell lines of non-brain endothelial origin showed that some AEDs such as vigabatrin, gabapentin, phenobarbitone, lamotrigine, carbamazepine, and phenytoin are not, or are only weak, P-gp substrates, questioning whether P-gp could be the primary reason for AED resistance (Crowe \& Teoh, 2006; Maines et al., 2005; Owen et al., 2001; Weiss et al., 2003). In contrast, Cucullo et al., compared phenytoin permeation in brain capillary endothelial cells from drug-resistant epileptic human brain tissue with that of commercially available human brain microvascular endothelial cells (Cucullo et al., 2007). They demonstrated that phenytoin permeation was 10-fold lower in endothelial cells from AED-resistant patients compared to purchased human endothelial cells. Although this comparison is flawed, inhibiting P-gp increased phenytoin permeation in the AED-resistant cells. Moreover, recent in vivo data, including our own studies, demonstrate that P-gp does limit AEDs from entering the brain (Brandt et al., 2006; Liu et al., 2007; Van Vliet et al., 2007). Using a drug-resistant epilepsy rat model, Potschka et al. showed that animals not responding to phenytoin exhibited 2-fold higher P-gp expression levels in brain capillaries compared to animals responding to treatment (Potschka et al., 2004). van Vliet et al. demonstrated that inhibiting P-gp counteracted phenytoin resistance, which reduced seizure occurrence in rats (Van Vliet et al., 2006). Marchi et al. supported these findings showing that patients with high blood-brain barrier P-gp expression had low brain levels of 
oxcarbazepine (Marchi et al., 2005). These studies demonstrate that, in drug-resistant epilepsy, certain, but not all AEDs have restricted access to the brain due to increased bloodbrain barrier P-gp, and that modulation of P-gp can enhance brain distribution of some AEDs such as phenytoin (Potschka \& Loscher, 2001; Van Vliet et al., 2006; Van Vliet et al., 2007).

In addition to P-gp, data indicate that BCRP plays a significant role in drug efflux at the blood-brain barrier. Recent studies show that both transporters, P-gp and BCRP, "team up" and work together to limit chemotherapeutic drugs from permeating across the blood-brain barrier and penetrating into the brain (Chen et al., 2009; De Vries et al., 2007). However, little information is available on the extent to which BCRP contributes to AED resistance and if Pgp and BCRP work in concert in AED efflux from the brain. Some studies found no upregulation of BCRP in human epileptogenic brain tissue and no evidence for BCRPmediated AED transport in vitro (Cerveny et al., 2006; Sisodiya et al., 2003), but other studies reported upregulation of BCRP expression in the microvasculature of epileptogenic brain tumors (Aronica et al., 2005; Vogelgesang et al., 2004) and in chronic epilepsy animal models (Van Vliet et al., 2005). More studies are needed to unequivocally clarify the role of BCRP, especially in conjunction with P-gp, in AED-resistant epilepsy.

Only little information is available on the multidrug resistance proteins (Mrps) in epilepsy. van Vliet et al. used the pilocarpine status epilepticus model in rats and found by immunohistochemistry and Western blotting that Mrp1 and Mrp2 protein expression was upregulated in astrocytes within several limbic structures including the hippocampus (Van Vliet et al., 2005). These findings were confirmed by Hoffmann et al., who also demonstrated Mrp2 upregulation in brain capillaries by immunohistochemistry following pilocarpine-induced status epilepticus (Hoffmann et al., 2006). In control rats, Mrp2 was barely detectable in the brain capillary endothelium, but in status epilepticus rats, Mrp2 staining was evident in brain capillary endothelial cells. MRP2 has also been found to be over-expressed in sclerotic hippocampal tissue of AED-resistant patients with mesial temporal lobe epilepsy (Aronica et al., 2004). In the same patient population, MRP1 expression was upregulated in glial endfoot processes around cerebral blood vessels. Observations of chronic epileptic rats showed that protein levels of Mrp1 and Mrp2 were also upregulated in blood vessels and this over-expression correlated with seizure frequency and reduced brain uptake of phenytoin (Van Vliet et al., 2005). However, phenytoin brain uptake was enhanced by the MRP inhibitor probenecid. While upregulation of mRNA was observed for Mrp1, 5, and 6, increased protein expression was only found for MRP1 and 2 in isolated capillary endothelial cells from patients with drug-resistant epilepsy (Dombrowski et al., 2001; Kubota et al., 2006). A time-course study revealed that 6-24 h after onset of a pilocarpine-induced status epilepticus in rats, mRNA of P-gp, Mrp1, and Mrp5 was decreased in hippocampus, amygdala, and the piriform cotex. This initial decrease in mRNA levels was followed by a $24 \mathrm{~h}$ period of normal mRNA expression and then increased mRNA levels about 4 days after status epilepticus (Kuteykin-Teplyakov et al., 2009). These findings are in contrast to an earlier study where P-gp mRNA levels in mouse hippocampus were increased by $85 \%$ 3-24 h after kainic acid-induced limbic seizures, but returned to control levels after $72 \mathrm{~h}$ (Rizzi et al., 2002). Treatment with AEDs for 7 days did not change P-gp mRNA expression (Rizzi et al., 2002). In the same study, the authors also used rats with spontaneous recurrent seizures 3 months after electrically induced status epilepticus. P-gp mRNA levels were increased 1.8- and 5-fold in the hippocampus and entorhinal cortex, 
respectively. Thus, changes in P-gp mRNA levels occur after both acute and chronic epileptic activity. The same authors (Rizzi et al., 2002) also used microdialysis and demonstrated that AED brain levels were significantly reduced. While a direct connection between blood-brain barrier P-gp levels and AED brain levels was not shown, it was concluded that seizure-induced changes in P-gp could contribute to AED resistance in epilepsy. Note that none of these studies provided data on transporter protein expression or activity.

\subsubsection{Transporter inhibition}

The discovery that drug efflux transporters are upregulated at the blood-brain barrier in AED-resistant patients suggested that transporter inhibition could overcome AED resistance in epilepsy. This notion was encouraged by studies that showed enhanced brain uptake of AEDs when co-administered with transporter inhibitors. Using verapamil and probenecid, Potschka et al. used microdialysis and demonstrated in healthy rats that P-gp and Mrp limit carbamazepine brain uptake (Potschka \& Loscher, 2001). A follow-up study showed that administration of the metabolic inhibitor sodium cyanide and the P-gp inhibitors verapamil and PSC833 into the frontal cortex significantly increased extracellular fluid concentrations of phenytoin. This indicated that P-gp limits phenytoin distribution into the brain under physiological conditions (Potschka \& Loscher, 2001). Similar observations were made with phenobarbital, lamotrigine, and felbamate (Potschka et al., 2002). Verapamil has also been used in case studies with AED-resistant patients (Iannetti et al., 2005; Summers et al., 2004). For example, the status epilepticus in an 11-year old boy who was first unresponsive to conventional AEDs disappeared after administration of verapamil i.v. (Iannetti et al., 2005). However, this anticonvulsive response could have been due to verapamil directly blocking neuronal calcium channels instead of inhibiting P-gp at the blood-brain barrier.

In 2005, tariquidar (XR9576), a non-competitive P-gp inhibitor was first used to block P-gp function. (Martin et al., 1999; Mistry et al., 2001). Tariquidar has a good oral bioavailability, long duration of action and low potential for toxic side effects, all of which make this a favourable P-gp inhibitor. For example, van Vliet et al. demonstrated that inhibiting P-gp with tariquidar significantly reduced seizure duration, frequency and severity, which improved phenytoin efficacy in a rat model for temporal lobe epilepsy. This suggested that combination of AEDs with a transporter inhibitor may be a promising therapeutic strategy for AED-resistant patients (Van Vliet et al., 2006). The same researchers also found that P-gp over-expression in the temporal hippocampus and parahippocampal cortex of chronic epileptic rats reduced phenytoin levels by about 30\% in these brain regions. Treating animals with tariquidar significantly increased phenytoin brain levels in regions with overexpressed P-gp (Van Vliet et al., 2007). Another group found that tariquidar restored the anticonvulsive activity of phenobarbital in drug-resistant rats (Brandt et al., 2006). These animal studies demonstrate that transporter inhibition increases AED blood and brain levels and improves seizure control.

Encouraged by animal studies and case reports that suggested transporter inhibition can be used to overcome AED resistance in epilepsy, clinical trials employing P-gp inhibitors were initiated. Currently, two trials using carvediol and verapamil to inhibit P-gp in AED refractory patients are ongoing (www.clinicaltrials.gov, \#NCT00524134, \#NCT01126307). However, while both carvedilol and verapamil are FDA-approved and readily available, neither drug is a highly specific nor potent P-gp inhibitor (Arboix et al., 1997; Takara et al., 
2004). In addition, to effectively inhibit over-expressed P-gp, high inhibitor plasma concentrations will be needed, which carries the risk of drug-drug interactions and toxic side effects (Pennock et al., 1991). In a recent study, add-on treatment with verapamil to improve seizure control in dogs with phenobarbital-resistant epilepsy had to be discontinued due to detrimental effects (Jambroszyk et al., 2011). Thus, since potent and specific inhibitors that can be safely given to patients are currently not available, transporter inhibition does not seem to be a viable treatment option in drug-resistant epilepsy at this point in time.

\subsubsection{Modulation of transporter regulation}

Targeting signalling pathways that regulate drug efflux transporters is another strategy to overcome transporter-mediated AED resistance. The advantages of this approach are threefold. First, modulating transporter regulation to increase AED brain delivery may allow fine tuning of the transporter. For example, modulating the molecular switches of a transporter may allow turning it off for a short, controlled period of time to deliver drugs into the brain, after which it can be turned on again. Second, preventing or blocking seizure-induced upregulation of transporters may normalise transporter expression and functional activity, and thus, prevent or block development of transporter-mediated AED resistance. Third, since transporter upregulation in epilepsy has been linked to increased seizure occurrence, prevention of transporter upregulation holds the promise of better seizure control. Thus, mapping the signalling pathways involved in efflux transporter upregulation at the bloodbrain barrier in epilepsy can help identify new targets that may potentially be used to overcome transporter-mediated AED resistance and improve seizure treatment.

Several signalling pathways have been identified that regulate P-gp, BCRP, and Mrp2 at the blood-brain barrier. For BCRP, the most recent signalling mechanisms include Nrf2, NfkB, COX-2, Pim-1 kinase, and the nuclear receptors CAR and AhR (Kalalinia et al., 2011; Singh et al., 2010; Tan et al., 2010; Wang et al., 2010). Of those, CAR and AhR have been shown to upregulate BCRP at the blood-brain barrier (Tan et al., 2010; Wang et al., 2010), which is the opposite of what one would want to improve AED delivery into the brain. Whether targeting any of the other pathways could be used as a therapeutic strategy in AED-resistant epilepsy is unknown and remains to be determined. Mrp2 is also regulated through nuclear receptors (PXR, FXR, CAR; (Bauer et al., 2008; Kast et al., 2002)), but the signalling that upregulates Mrp2 in epilepsy is unknown.

Most information on transporter regulation is available for P-gp, where signalling pathways have been shown to be present in various tissues (liver, kidney, intestine; (Ho \& PiquetteMiller, 2006; Nawa et al., 2010; Thevenod et al., 2000). They also involve various signalling molecules: inflammatory mediators including TNF-a, ET-1, IL1- $\beta$, IL-6, NO; COX-2 (Dixit et al., 2005; Goralski et al., 2003; Nawa et al., 2010; Patel et al., 2002; Poller et al., 2010; Sukhai et al., 2001; Von Wedel-Parlow et al., 2009), nuclear receptors PXR, CAR, AhR, and GR (Bauer et al., 2004; Bauer et al., 2007; Geick et al., 2001; Narang et al., 2008; Wang et al., 2011; Wang et al., 2010), protein kinase C (Bauer et al., 2007; Chambers et al., 1990a; Chambers et al., 1990b; Hartz et al., 2004; Miller et al., 1998; Rigor et al., 2010), and NFkB (Bauer et al., 2007; Bentires-Alj et al., 2003; Kim et al., 2011; Liu et al., 2008; Thevenod et al., 2000; Yu et al., 2008). These pathways have been found in several diseases including Alzheimer's disease, HIV, and diabetes (Hartz et al., 2010; Hayashi et al., 2006; Nawa et al., 2010). 
One pathway that involves glutamate signalling through the NMDA receptor (NMDAR) followed by cyclooxygenase -2 (COX-2) and prostaglandin E receptor 1 (EP1) activation seems to be critical for seizure-induced upregulation of P-gp (Figure 3, (Bankstahl et al., 2008; Bauer et al., 2008; Pekcec et al., 2009; Zhu \& Liu, 2004)). During seizures, neurons release high amounts of the excitatory neurotransmitter glutamate, which can reach interstitial brain concentrations of $10-100 \mu \mathrm{M}$ for a short period of time (Ronne-Engstrom et al., 1992; Ueda \& Tsuru, 1995). Zhu and Liu were the first to connect glutamate with P-gp upregulation at the blood-brain barrier. They found that glutamate increased P-gp expression and activity in rat brain microvessel endothelial cells and suggested that activation of the NMDAR plays a critical role in glutamate-mediated P-gp upregulation (Zhu \& Liu, 2004). Consistent with this, Bauer et al. demonstrated that exposing isolated rat and mouse brain capillaries to glutamate increased P-gp expression and activity (Bauer et al., 2008). It was shown that glutamate signalling through NMDAR and COX-2 upregulates blood-brain barrier P-gp, and that COX inhibition prevented P-gp upregulation suggesting that AED brain uptake can be enhanced by COX inhibition. Bankstahl et al. confirmed glutamate involvement in seizure-induced P-gp over-expression and that blocking NMDAR prevents P-gp upregulation and neuronal damage in vivo (Bankstahl et al., 2008). Another study showed that pre-treatment with celecoxib, a specific COX-2 inhibitor, prevented seizure-induced P-gp upregulation in rat brain capillaries (Zibell et al., 2009), and yet another study demonstrated that pre-treatment with celecoxib for 6 days followed by administration of phenobarbital for 16 days reduced the frequency of spontaneous recurrent seizures and restored the anticonvulsant effect of phenobarbital in AED-resistant epileptic rats (Schlichtiger et al., 2010). van Vliet et al. evaluated the use of the COX-2 inhibitors SC58236 and NS-398 in rats with recurrent spontaneous seizures. They found that 2-week treatment with these COX-2 inhibitors prevented P-gp upregulation and enhanced phenytoin brain uptake in chronic epileptic rats (Van Vliet et al., 2010). While these studies are promising, COX-2 inhibitors bear the risk of severe cardio- and cerebrovascular side effects (Mukherjee, 2001; Stollberger \& Finsterer, 2003). In addition, it has been demonstrated that COX-2 inhibition can lead to increased seizure frequency and mortality in epileptic rats (Holtman et al., 2010). Thus, although COX-2 inhibition may reduce AED resistance in animal models, it may not be a valid target in the clinic over the long term.

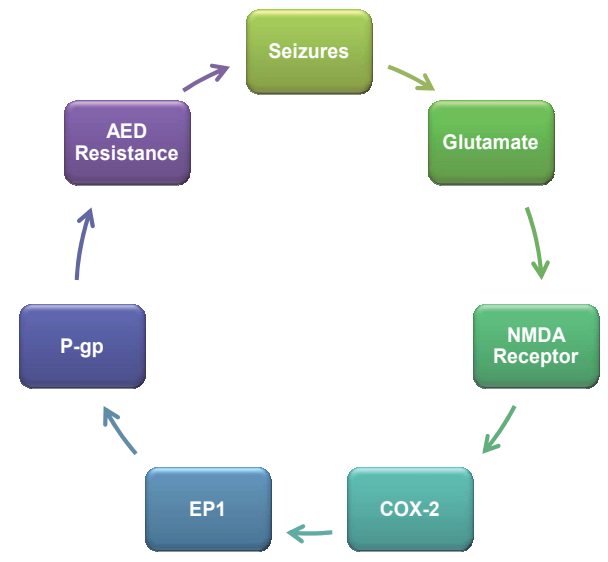

Fig. 3. Glutamate - NMDAR1 - COX-2 - EP1 Signaling Pathway 
Another signalling protein involved in glutamate-mediated P-gp upregulation at the bloodbrain barrier is the EP1 receptor. EP1 is activated by prostaglandin E2, the main product of COX-2, and was tested as potential target to prevent transporter upregulation in epilepsy. Pekcec et al. found that EP1 is a key signalling protein in the pathway that drives P-gp upregulation during seizures (Pekcec et al., 2009). Studies using the EP1 inhibitors SC-51089 and misoprostol showed that SC-51089 decreased seizure severity in rats when given prior to electrical kindling, but it also prolonged seizure duration at higher doses, whereas misoprostol decreased duration of motor seizure activity (Fischborn et al., 2010).

Together, these studies show that glutamate released during seizures mediates P-gp upregulation through NMDAR, COX-2, and EP1 and that these signalling proteins could potentially be used as therapeutic targets to reduce AED-resistance. Whether this pathway also signals upregulation of other blood-brain barrier proteins is unknown at this time.

\subsection{The metabolic blood-brain barrier}

Xenobiotic metabolism is a 3-phase process during which low polar molecules (e.g., drugs) are enzymatically converted to polar molecules that are then excreted from the body mostly through bile, faeces, or urine. Most chemicals are pharmacologically or toxicologically inactivated during metabolism, only some are transformed into active metabolites. The liver is recognized as the main site of biotransformation, but extrahepatic tissues such as the kidney, lung, intestine, skin, and brain also contribute to drug metabolism.

The processes involved in the biotransformation of drugs are classified into phase I (functionalisation) and phase II (conjugation) reactions that are followed by phase III excretion of the metabolite (Figure 4). Substrates of phase I enzymes are in general lipophilic and undergo functionalisation reactions such as monooxygenation, dealkylation, reduction, aromatisation, or hydrolysis. The modified molecules are substrates for phase II enzymes, which conjugate the functional group with a polar compound, such as an amino acid, sulphate, glutathione, or a sugar (Minn et al., 1991). In the last phase III step, functionalised and conjugated xenobiotics are excreted from cells by efflux transporters.

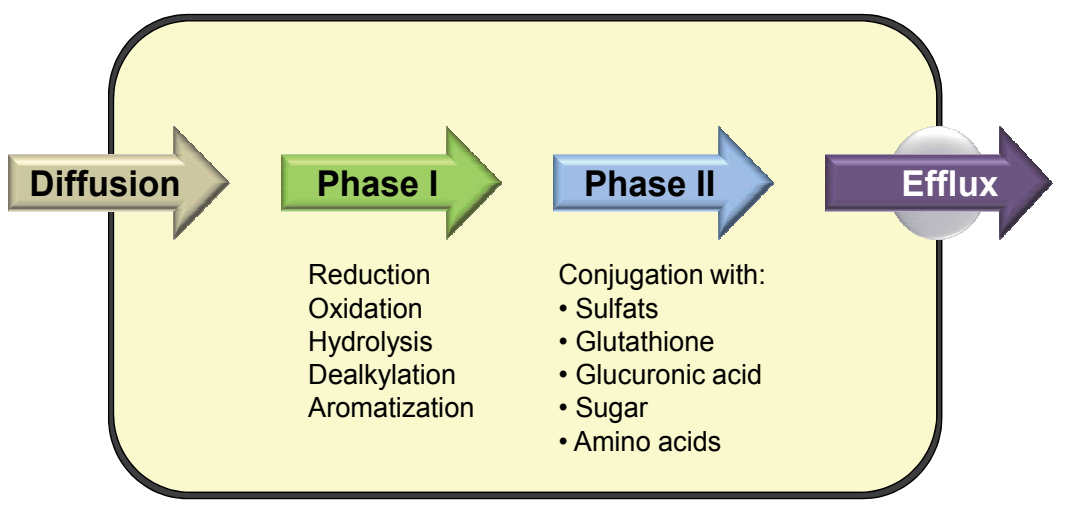

Fig. 4. Schematic of 3-phase drug metabolism and excretion

Most CNS drugs, including AEDs, have to cross the blood-brain barrier and penetrate into the brain parenchyma to reach their target sites. En passage across the barrier and within the brain, drugs can undergo inactivation and elimination comparable to hepatic drug 
metabolism. In the brain, the following phase I enzymes have been identified: monoamine oxidases, CYP P450, NADPH-CYP P450 reductase, and epoxide hydrolases (Chat et al., 1998; Dutheil et al., 2010; Ghersi-Egea et al., 1998; Ghersi-Egea et al., 1988; Ghersi-Egea et al., 1993; Minn et al., 1991; Ravindranath et al., 1990). Phase II enzymes identified in the brain include UDP-glucuronosyltransferase (UGT), phenol sulfotransferase (PST), and GST (Dutheil et al., 2010; Ghersi-Egea et al., 1998; Ghersi-Egea et al., 1988; Ghersi-Egea et al., 1993; Minn et al., 1991). Several phase I and II enzymes have been found in the brain capillary endothelium, where they possibly form a metabolic barrier for drugs en route into the brain (Dutheil et al., 2010; Ghersi-Egea et al., 1998; Ghersi-Egea et al., 1993; Minn et al., 1991; Stamatovic et al., 2008). Several reports on metabolism-coupled efflux transport (phase III) suggest that biotransformation of drugs and efflux of the metabolites are part of barrier function. Together, the rodent and human brain, including brain microvessels forming the bloodbrain barrier, express enzymes and transporters that are part of the detoxification pathways that affect metabolism of therapeutic drugs. In addition, AED elimination by coupling of two important biological processes - metabolism and efflux transport - could contribute to AED resistance and intractable epilepsy.

\subsubsection{Metabolism of AEDs and the metabolic barrier in epilepsy}

Phase I. CYP P450 enzymes are responsible for most phase I metabolic reactions and have the greatest impact on the biotransformation of therapeutic drugs. CYPs form a large and functionally diverse superfamily of enzymes that are found throughout various species ranging from bacteria to humans. In humans, the majority of CYPs are expressed along the inner plasma membranes of mitochondria and the endoplasmic reticulum. Although a distinct group of CYPs (CYP11A1, 11B1, 11B2, 17A1, 21A2) is involved in steroid hormone synthesis in humans (Hrycay \& Bandiera, 2009), most CYP enzymes contribute primarily to the elimination of endogenous and exogenous substrates through oxidation to enhance their excretion from the body. By-in-large, CYP-mediated metabolism occurs in the liver and contributes to the "first pass effect" of orally administered drugs. In the liver, several CYPs exist as allelic or genetic variants and such CYP polymorphisms have been shown to influence the plasma concentration of some AEDs. CYPs are also expressed in the kidney and renal excretion is an important elimination route, particularly for most novel AEDs.

With regard to epilepsy it is noteworthy that many AEDs are metabolised by CYPs (Table 1). CYP2C9 and CYP2C19 are the two major enzymes involved in AED metabolism including diazepam, phenobarbital, phenytoin, and valproic acid (Table 1; (Klotz, 2007)). Several studies demonstrated differences in the biotransformation of these AEDs depending on the underlying CYP genotype. For example: phenytoin metabolism depends on the allelic composition of the gene encoding for CYP2C19 and CYP2C9. Several mutated alleles of these genes are known. Thus, based on genotype, poor phenytoin metabolisers can be distinguished from phenytoin hypermetabolisers and their frequency distributions vary between different ethnic populations (Klotz, 2007).

Regarding CYPs in the brain, in vitro and in vivo functional expression of CYPs has been detected in various CNS cell types from different species. CYP expression in distinct CNS cell populations is variable but can be as high as in the liver (Bhagwat et al., 2000). Importantly, it has been shown that CYPs are functionally active at the blood-brain barrier (Dutheil et al., 2010). 


\begin{tabular}{|l|l|}
\hline DRUG & ENZYME/PATHWAY \\
\hline Carbamazepine & CYP3A4, mEH1 for carbamazepinee-10, 11-epoxide \\
Ethosuxemid & CYP3A4?, 10-20\% renal \\
Phenobarbital & CYP2C19, hydroxylation, glucuronidation, 25\% renal \\
Phenytoin & CYP2C9, CYP2C19 \\
Valpoate & CYP2C9, glucuronidation, oxidation \\
Diazepam & CYP2C19, CYP3A4 \\
Felbamate & 40-60\% renal, hydroxylation, glucuronidation \\
Gabapentin & Renal \\
Lamotrogine & Glucuronidation, 8\% renal \\
Levetirazepam & $66 \%$ renal, hydrolyse \\
Oxcabazepine & Reduction to active metabolite that is glucuronidated \\
Pregabalin & $98 \%$ renal \\
Tiagabine & CYP3A4, 25\% renal \\
Topiramate & $60-80 \%$ renal, oxidation, hydrolysis, glucuronidation \\
Vigabatrin & $60-80 \%$ renal \\
Zonizamide & CYP3A4, N-acetylation, glucuronidation, 30\% renal \\
\hline
\end{tabular}

Table 1. Antiepileptic drugs and their route of biotransfomation (modified from Klotz, 2007)

In the human brain, $20 \mathrm{CYP}$ isoforms have been identified so far: CYP1A1, 1A2, 1B1, 2B6, 2C8, 2D6, 2E1, 3A4, 3A5, 8A1, 11A1, 11B1, 11B2, 17A1, 19A1, 21A2, 26A1, 26B1, 27B1, and 46A1 (Dutheil et al., 2010). The exact expression pattern within the CNS depends on the particular CYP and greatly varies between different brain cells. In a recent study, CYP mRNA expression levels were measured in a human brain microvessel cell line and in human microvessels isolated from surgically removed brain tissue from epileptic patients or patients with brain tumours (Dauchy et al., 2008). The authors found mRNA expression of CYP2U1, CYP2S1, CYP2R1, CYP2B6, CYP2E1, CYP1A1, CYP2D6, CYP1B1, CYP2J2, CYP1A2, and CYP2C8. In another study, Gosh et al. used commercially available human microvascular cerebral endothelial cells and found mRNA expression of CYP1A1, 1B1, 2A6, 2B6 2C, 2C9, 2E1, 2J2, 3A4, 4A11, 11b, CYP3A5, 4B1, C1, 21A, and 51A1 (Ghosh et al., 2010). The cells that were used for this study originated from surgically resected brain specimens of drug-resistant epileptic patients, brain specimens resected from aneurism domes, commercially available human microvascular cerebral endothelial cells (used as control), and from human umbilical vein endothelial cells (used as control). mRNA expression from 11 of the 16 CYPs was increased in endothelial cells from epileptic tissue compared to microvascular cerebral endothelial cells. Although this comparison may be flawed due to the different nature of these cells, mRNA expression in endothelial cells from drug-resistant epileptic patients was not different compared to brain specimens resected from aneurism domes without seizures (Ghosh et al., 2010). This result argues against a seizure effect on the regulation of blood-brain barrier CYPs. However, it is clear from these findings that more detailed and accurate studies with appropriate controls are needed.

Overall, expression profiles, tissue and cellular distribution, and relative expression of CYP enzymes seem to depend on study design and the models used. Therefore, additional research is needed to clarify the importance of individual CYPs at the blood-brain barrier under both physiological and epileptic conditions. 


\section{CYP Regulation in the CNS}

Carbamazepine induces CYP3A4 protein and mRNA expression in human brain endothelial cells and hepatocytes (Ghosh et al., 2010; Luo et al., 2002). But carbamazepine is metabolized by CYP3A4 (, Table 1), and high expression of CYP3A4 protein was found in endothelial cells isolated from surgically resected epileptic brain tissue (Ghosh et al., 2011). Neuronal CYP3A4 expression has also been demonstrated in brain sections by immunostaining from patients with temporal lobe epilepsy, tuberous sclerosis, or cavernous angioma, all of whom had intractable epilepsy. In these samples, CYP3A4 was rarely co-localised with the astrocytic marker GFAP (Ghosh et al., 2011). Carbamazepine given to cells that derived from resected epileptic brain tissue underwent metabolism at an extent similar to what was observed in hepatocytes. Thus, increased CYP3A4 expression and metabolic function could be characteristic for endothelial cells in epilepsy, which could contribute to AED resistance. As mentioned before, AEDs such as carbamazepine act as strong inducers of hepatic and blood-brain barrier CYP expression, thereby influencing the pharmacokinetics of other drugs. Other AEDs have also been reported to increase CYP expression at the blood-brain barrier and exposure of primary rat brain astrocytic cultures to phenytoin increased Cyp2c29 levels (Volk et al., 1995). Moreover, phenytoin was metabolised by the microsomal fraction of astrocyte cultures and chronic treatment of mice with phenytoin resulted in increased levels of phenytoin metabolites in the brain (Volk et al., 1988). These findings support the idea of dynamic CYP regulation at the blood-brain barrier by AED exposure. In general, regulation of CYPs at the blood-brain barrier could be independent from that in the liver. Support for this comes from studies in alcoholics. Levels of CYP2D6 protein were elevated in the brains of alcoholics compared to non-alcoholics (Dutheil et al., 2010; Miksys \& Tyndale, 2004). Particularly high CYP2D6 levels were detected in the putamen, globus pallidus, and substantia nigra, but interestingly, CYP2D6 was not elevated in the liver (Dutheil et al., 2010). Nuclear receptors that act as transcription factors control regulation of CYPs and ABC transporters in the CNS. In the human brain, several nuclear receptors have been detected that could control CYP regulation, including AhR, PXR, FXR, CAR, LXRß, RXRa and $\beta$, PPAR- $\alpha,-\delta$, and $-\gamma$ (Dutheil et al., 2009; Nishimura et al., 2004). For example, Dauchy et al. showed that the AhR agonist TCDD increased mRNA expression of CYP1A1 and CYP1B1 in the human cell line hCMEC/D3 (Dauchy et al., 2008). Thus, it is possible that nuclear receptors could be involved in the regulation of CYPs at the blood-brain barrier in epilepsy (Dauchy et al., 2008; Dauchy et al., 2009; Ghosh et al., 2010). This idea is supported by the fact that metabolites of CYP2J2, which is expressed in brain endothelial cells in epilepsy, activate the nuclear receptors PPAR-a (NR1C1) and PPAR- $\gamma$ (NR1C3). Future studies are required to address CYP regulation at the blood-brain barrier in health, disease (e.g., epilepsy), and during pharmacotherapy (e.g., AED treatment).

Phase II \& III. Metabolism-driven efflux transport, i.e., coupling of phase II and III, has been demonstrated in the liver. Analogous findings from studies at the blood-CSF-barrier show coupling of metabolism and efflux transport also in the CNS. Using cultured rat choroid plexus epithelial cells in vitro, Strazielle and Ghersi-Egea demonstrated the presence of a metabolism-driven efflux mechanism for 1-naphthol, a cytotoxic, lipophilic model compound (Strazielle \& Ghersi-Egea, 1999). The authors showed that UGT metabolised 1naphthol in situ into a glucurono-conjugate (phase II) that was excreted by an efflux transporter (phase III). In this regard, MRPs have been implicated in cellular export of various glutathione, glucuronide, and sulfate conjugates compounds, and several other 
endogenous and xenobiotic compounds (Gerk \& Vore, 2002; Jedlitschky et al., 1996; Loe et al., 1996; Oude Elferink \& Jansen, 1994). Although Mrp involvement has not directly been shown in Strazielle and Ghersi-Egea's study, Mrp-mediated efflux of the 1-naphthol glucurono-conjugate seems likely as the export was sensitive to the Mrp inhibitor probenecid. Thus, this study demonstrated the biological relevance of metabolism-driven efflux in the brain (Strazielle \& Ghersi-Egea, 1999). However, in the human brain, UGTmediated metabolism of 1-naphthol is less prominent compared to rat brain, and therefore, species differences should be considered (Ghersi-Egea et al., 1993). Activity of another phase II enzyme, GST, seems to be more relevant for human metabolism (Ghersi-Egea et al., 1993). Metabolism-driven transport was shown for GSTn and Mrp1 by Leslie et al., who demonstrated plasma membrane co-localisation of Mrp1 and GSTn in H69AR cells and found that functional GSTп was required for Mrp1-mediated transport (Leslie et al., 2004). mRNA and protein expression of the GST isoform $\Pi$ has been demonstrated in isolated rat and mouse brain capillaries, where GST $\Pi$ is predominantly localized in the cytoplasm and the luminal plasma membrane of brain capillary endothelial cells, and to a large extent, colocalises with Mrp2 in the membrane (Bauer et al., 2008). Consistent with regulation by the nuclear receptor PXR, GSTп protein expression increased in membranes from rat brain capillaries exposed to PCN or dexamethasone, and in capillary membranes from rats dosed with PCN. Immunoblotting of the capillary membrane fraction from hPXR transgenic mice dosed with rifampin further showed enhanced GSTп expression. GSTп and Mrp2 upregulation occurred in parallel, suggesting coordinated regulation of phase-II metabolism and phase-III efflux, i.e. Mrp2-mediated transport (Bauer et al., 2008). While these studies provide first insight into the regulation of both GSTп and Mrp2 in brain capillaries, direct proof of metabolism-coupled efflux transport of chemicals at the blood-brain barrier remains to be shown. Thus, metabolism-coupled excretion of CNS drugs, such as AEDs, by efflux transporters seems likely, but requires further studies. Such studies would have to take into consideration the specific conditions at the human blood-brain barrier. For example, human cerebral microvessels show absence of glucuronidation, low NADPH CYP reductase activity, high GST activity, and pronounced epoxide hydrolase activity (GhersiEgea et al., 1993). Understanding metabolising enzymes, specifically at the human bloodbrain barrier with respect to their physiological function, regulation in health and disease, and interplay with efflux transporters will allow assessing their impact on drug delivery to the brain, particularly in epilepsy.

\subsection{Blood-brain barrier leakage in epilepsy}

Seizures are accompanied by impaired blood-brain barrier integrity. This has been observed before, during and after seizures in both experimentally induced seizures in animals as well as in epileptic patients (Cornford \& Oldendorf, 1986; Horowitz et al., 1992; Mihaly \& Bozoky, 1984; Nitsch \& Klatzo, 1983; Padou et al., 1995). As a consequence, impaired bloodbrain barrier integrity causes transient barrier leakage, which allows entry of blood borne molecules into the brain (Ndode-Ekane et al., 2010; Seiffert et al., 2004; Sokrab et al., 1989; Van Vliet et al., 2007). It has been shown that seizure duration correlates with reduced barrier function (Cornford \& Oldendorf, 1986), and it has been demonstrated that increased blood-brain barrier permeability in epilepsy is limited to anatomically specific brain regions (Bradbury, 1979; Cornford et al., 1998; Nitsch \& Klatzo, 1983; Oztas \& Sandalci, 1984). Interestingly, brain regions with increased barrier permeability are often anatomically 
congruent with the brain regions that are implicated in the development and propagation of seizures. Consistent with this observation, extravasation of blood components into the brain correlates with increased excitability, occurrence of seizures, and epilepsy progression (Friedman et al., 2009; Marchi et al., 2007; Ndode-Ekane et al., 2010; Oby \& Janigro, 2006; Seiffert et al., 2004; Tomkins et al., 2008; Van Vliet et al., 2007).

Cause of Blood-Brain Barrier Leakage by Seizures. Studies conducted over the last 3 decades indicate that possibly 3 mechanisms could be involved in causing blood-brain barrier leakage in epilepsy: blood pressure, pinocytosis, and seizure-induced inflammation.

\section{Blood Pressure}

The first studies conducted in the 1970s showed that arterial blood pressure is involved in seizure-associated blood-brain barrier leakage (Nitsch \& Klatzo, 1983). Several studies unequivocally demonstrated that hypertension has detrimental effects on blood-brain barrier integrity and contributes to barrier leakage (Cornford \& Oldendorf, 1986; Lee \& Olszewski, 1961; Petito et al., 1977; Westergaard, 1980). Johansson summarized three factors responsible for increased blood-brain barrier permeability: (1) maximal arterial blood pressure, (2) duration of maximal arterial blood pressure, and (3) total increase in blood pressure (Johansson, 1981). The detailed mechanism through which increased blood pressure contributes to barrier leakage is unclear, but a working hypothesis postulates the following (Petito et al., 1977): Neuronal hyperactivity (seizures) leads to increased metabolism, and to an increased nutrient and oxygen demand in the involved brain regions. In turn, cerebral blood flow rises and large cerebral arteries dilate, which leads to increased blood pressure in brain capillaries, small arteries and veins (Ndode-Ekane et al., 2010), and triggers barrier leakage. Consistent with this, extravasation of blood albumin into the brain was found specifically in regions with more EEG spiking activity in humans (Cornford et al., 1998). However, the duration of increased blood pressure is critical and determines the severity of barrier leakage. Thus, more severe seizures that are followed by prolonged blood pressure elevations result in a higher increase in barrier permeability (Oztas \& Kaya, 1991; Oztas \& Sandalci, 1984). This is also supported by studies where both increased arterial blood pressure and subsequently induced barrier leakage were prevented by cervical cordotomy (Schaefer et al., 1975; Westergaard et al., 1978).

\section{Pinocytosis}

It was hypothesized that pinocytosis is involved in transport across the capillary endothelium, thus, affecting barrier permeability (Palade, 1961). The pinocytosis rate at the blood-brain barrier is low, which contributes to a tight barrier endothelium. However, Petito et al. observed that seizure-induced blood-brain barrier leakage correlates with increased micropinocytosis (Petito et al., 1977). In an elegant study using intravenous HRP injections in adult male rats with seizures, the authors made two important findings: (1) Brain capillary vesicles from animals that suffered seizures did contain HRP compared to vesicles from control animals that did not contain HRP; (2) the number of HRP-containing vesicles was higher directly after seizures (within $30 \mathrm{sec}$ of last seizure (Petito et al., 1977)). From these observations the authors concluded that an increased micropinocytosis rate during and shortly after seizures increases blood-brain barrier permeability and counteracts barrier function. Nitsch and Hubauer confirmed these studies and showed in kainic acid-injected rats that blood-brain barrier opening was due to increased transendothelial pinocytosis, while tight junctions stayed intact (Nitsch \& Hubauer, 1986). 


\section{Seizure-induced Inflammation}

Another factor causing barrier leakage in epilepsy is seizure-induced inflammation that could be enhanced by extravasation of blood-borne components into the brain. It has been shown that blood-brain barrier permeability is increased by inflammatory mediators, including histamine, substance $P$, endothelin-1, bradykinin, VEGF, TGFß, IL1ß, TNFa, INFY, PGE2, PGF2a, chemokines, free radicals, and other factors such as metalloproteinases, thrombin, amyloid- $\beta$, intracellular calcium, and leukocytes that directly interact with endothelial cells (Stamatovic et al., 2008). Only limited information is available on how these factors alter the blood-brain barrier but some, e.g., IL1ß and chemokines, seem to exclusively affect paracellular permeability (Stamatovic et al., 2008). It is currently unknown if secretion of these factors is a direct consequence of epileptic seizures. In addition, depending on epilepsy aetiopathology, the composition of the inflammatory "cocktail" and the contribution of individual inflammatory mediators to blood-brain barrier damage could vary significantly. The effect of inflammation on barrier permeability is context-dependent, complex and not well understood. It likely depends on the model, dose, time and location of the inflammatory mediators involved.

One hypothesis that could explain some of the phenomena observed at the blood-brain barrier in epilepsy is that seizure-released glutamate activates signalling reducing barrier integrity and increasing permeability. Glutamate release occurs during seizures at sites in the brain with excessive neuronal activity. On the one hand, high glutamate levels are cytotoxic, which contributes to brain damage. On the other hand, subtoxic glutamate levels trigger molecular processes such as local release and activation of matrix-degrading enzymes that breach integrity (Michaluk \& Kaczmarek, 2007; Nishijima et al., 2010). It is possible that glutamate-initiated signalling and inflammatory mediators cause barrier leakage and breakdown in epilepsy. Such events would allow extravasation of blood-borne compounds. Whether this scenario is part of seizures remains to be shown.

Consequences of Blood-Brain Barrier Leakage in Epilepsy. Epilepsy is often a consequence of a prior brain insult (e.g., traumatic brain injury, stroke) and seizures are a symptom of an underlying brain disorder (e.g., brain tumour, Alzheimer's disease, brain inflammation; (Marchi et al., 2006; Salazar et al., 1985; Tomkins et al., 2011). Although the factors that are involved in the development of epilepsy remain unclear, impaired barrier function is common after an initial brain insult and likely contributes to epilepsy pathology. This particular topic has recently attracted major interest in the epileptology field.

It has been shown in patients that brain injury, post-ischemic or vascular inflammation often cause seizures and barrier leakage (Stamatovic et al., 2008; Tomkins et al., 2011; Tomkins et al., 2008). The blood-brain barrier is also impaired in epileptic patients and in seizure animal models, and consequently, it has been postulated that barrier leakage is involved in epilepsy aetiology (Friedman et al., 2009; Marchi et al., 2007; Ndode-Ekane et al., 2010; Oby \& Janigro, 2006; Seiffert et al., 2004; Tomkins et al., 2011; Tomkins et al., 2008; Van Vliet et al., 2007). In addition, it has been shown that osmotic barrier opening causes seizures (Oby \& Janigro, 2006). However, not all implications of barrier opening have been studied. It is known that intra-arterial injection of hyperosmotic mannitol in patients and rodents results in EEG changes and induces seizures (Fieschi et al., 1980). In a recent study, Marchi et al (2007) observed seizures in patients undergoing osmotic barrier opening for delivering chemotherapeutics to treat brain lymphomas. In $25 \%$ of patients, seizure onset occurred 
immediately after barrier opening. Using a pig model, the authors demonstrated that seizure occurrence correlated with barrier opening and was neither attributed to the existing brain lymphoma nor to chemotherapy (Marchi et al., 2007).

The molecular and cellular events that are triggered by barrier opening and that result in seizures and neuronal hyperactivity are a matter of research. Rigau et al. (2007) demonstrated loss of functional tight junctions and immunoglobulin leakage into the brain in surgically resected hippopcampal tissue from AED-resistant epilepsy patients (Rigau et al., 2007). Additional evidence from rodents and resected epileptogenic human brain tissue shows that extravasation of albumin into the brain triggers epileptogenesis (Friedman et al., 2009; Ivens et al., 2010). It was shown that astrocytes incorporated extravasated albumin, which induced proepileptogenic transformations, including reduced expression of potassium and aquaporin channels and gap junction proteins, impairment of astrocytic glutamate metabolism, and increased release of pro-inflammatory mediators (Friedman et al., 2009). All these changes had detrimental effects on seizure threshold and susceptibility (Friedman et al., 2009). One could postulate that seizures or other factors that induce barrier leakage trigger albumin extravasation with subsequent astrocytic transformation eventually causing seizures. Such a scenario implies a pernicious feedback loop where seizures drive barrier leakage leading to more seizures. Although this hypothesis is a matter of discussion, many epileptologists are convinced that 'seizures beget seizures' and that epilepsy has a progressive nature (Hauser \& Lee, 2002). In this regard, alterations of the blood-brain barrier and extravasation of blood-borne compounds could be a critical part of epilepsy pathology that could potentially be a target for new therapies.

\section{Conclusions}

Research over the last century demonstrated a key role of the blood-brain barrier in the development of epilepsy and AED resistance. Despite the advances that have been made, what we currently know about AED resistance is mostly limited to descriptive observations rather than understanding of the mechanisms underlying the disease. We know that the blood-brain barrier is altered in epilepsy including changes in transporters, metabolic enzymes, and tight junctions. We also know that transporters, enzymes and tight junctions are affected by and/or contribute to epilepsy pathology. Yet, whether each of these molecular players is part of a cause-effect jigsaw puzzle and how each of the pieces fit together is unclear. Thus, AED resistance in epilepsy remains an unsolved clinical problem. To solve this problem future studies will have to address the mechanism of AED resistance at the molecular level taking all aspects into account in a "big picture approach" rather than focusing on one single piece of the puzzle. The stimuli of blood-brain barrier transporter, enzyme and tight junction regulation in epilepsy will have to be identified and the detailed chain of signalling events will have to be unravelled. Such information will provide novel targets and therapeutic strategies that hold the promise to advance this research field and eventually improve treatment of patients with AED-resistant epilepsy.

\section{Acknowledgments}

We thank Britt Johnson for editorial assistance. This work was supported by UMN startup funds for A.M.S.H. and B.B. 


\section{References}

Aird, R.B. (1939). Mode of action of brilliant vital red in epilepsy. Archives of Neurology and Psychiatry Vol.42, pp.700-723

Al-Anizy, M. et al. (2006). Cytochrome P450 Cyp4x1 is a major P450 protein in mouse brain. FEBS J, Vol.273, No.5, (Mar), pp.936-947, ISSN 1742-464X

Arboix, M. et al. (1997). Multidrug resistance-reversing agents increase vinblastine distribution in normal tissues expressing the P-glycoprotein but do not enhance drug penetration in brain and testis. Journal of Pharmacology and Experimental Therapeutics, Vol.281, No.3, (Jun), pp.1226-1230, ISSN 0022-3565

Aronica, E. et al. (2004). Expression and cellular distribution of multidrug resistance-related proteins in the hippocampus of patients with mesial temporal lobe epilepsy. Epilepsia, Vol.45, No.5, (May), pp.441-451, ISSN 0013-9580

Aronica, E. et al. (2005). Localization of breast cancer resistance protein (BCRP) in microvessel endothelium of human control and epileptic brain. Epilepsia, Vol.46, No.6, (Jun), pp.849-857, ISSN 0013-9580

Awasthi, S. et al. (2005). RLIP76, a non-ABC transporter, and drug resistance in epilepsy. BMC Neurosci, Vol.6, pp.61, ISSN 1471-2202

Bankstahl, J.P. et al. (2008). Glutamate is critically involved in seizure-induced overexpression of P-glycoprotein in the brain. Neuropharmacology, Vol.54, No.6, (May), pp.1006-1016, ISSN 0028-3908

Bauer, B. et al. (2004). Pregnane X receptor up-regulation of P-glycoprotein expression and transport function at the blood-brain barrier. Molecular Pharmacology, Vol.66, No.3, (Sep), pp.413-419, ISSN 0026-895X

Bauer, B. et al. (2007). Tumor necrosis factor alpha and endothelin-1 increase P-glycoprotein expression and transport activity at the blood-brain barrier. Molecular Pharmacology, Vol.71, No.3, (Mar), pp.667-675, ISSN 0026-895X

Bauer, B. et al. (2008). Seizure-induced up-regulation of P-glycoprotein at the blood-brain barrier through glutamate and cyclooxygenase-2 signaling. Molecular Pharmacology, Vol.73, No.5, (May), pp.1444-1453, ISSN 1521-0111)

Begley, D.J. (2004). Delivery of therapeutic agents to the central nervous system: the problems and the possibilities. Pharmacology and Therapeutics, Vol.104, No.1, (Oct), pp.29-45, ISSN 0163-7258

Bentires-Alj, M. et al. (2003). NF-kappaB transcription factor induces drug resistance through MDR1 expression in cancer cells. Oncogene, Vol.22, No.1, (Jan 9), pp.90-97, ISSN 0950-9232

Bercel, N.A. (1955). Diuretics in therapy of epilepsy; their use for the potentiation of anticonvulsant drugs. California Medicine, Vol.82, No.2, (Feb), pp.107-110, ISSN 0008-1264

Betz, A.L. et al. (1980). Polarity of the blood-brain barrier: distribution of enzymes between the luminal and antiluminal membranes of brain capillary endothelial cells. Brain Research, Vol.192, No.1, (Jun 16), pp.17-28

Beuckmann, C. et al. (1995). Induction of the blood/brain-barrier-associated enzyme alkaline phosphatase in endothelial cells from cerebral capillaries is mediated via cAMP. European Journal of Biochemistry, Vol.229, No.3, (May 1), pp.641-644, ISSN 0014-2956 
Bhagwat, S.V. et al. (2000). Multiple forms of cytochrome P450 and associated monooxygenase activities in human brain mitochondria. Biochemical Pharmacology, Vol.59, No.5, (Mar 1), pp.573-582, ISSN 0006-2952

Boado, R.J. et al. (2004). Developmental regulation of the rabbit blood-brain barrier LAT1 large neutral amino acid transporter mRNA and protein. Pediatric Research, Vol.55, No.4, (Apr), pp.557-560, ISSN 0031-3998

Bolwig, T.G. et al. (1977). Acute hypertension causing blood-brain barrier breakdown during epileptic seizures. Acta Neurologica Scandinavica, Vol.56, No.4, (Oct), pp.335-342, ISSN 0001-6314

Bradbury, M.W.B. (1979) The Concept of a Blood-Brain Barrier. John Wiley, Chichester.

Brandt, C. et al. (2006). The multidrug transporter hypothesis of drug resistance in epilepsy: Proof-of-principle in a rat model of temporal lobe epilepsy. Neurobiology of Disease, Vol.24, No.1, (Oct), pp.202-211, ISSN 0969-9961

Braun, D. et al. (2011). Developmental and cell type-specific expression of thyroid hormone transporters in the mouse brain and in primary brain cells. Glia, Vol.59, No.3, (Mar), pp.463-471, ISSN 1098-1136

Brightman, M.W. \& Reese, T.S. (1969). Junctions between intimately apposed cell membranes in the vertebrate brain. Journal of Cell Biology, Vol.40, No.3, (Mar), pp.648-677

Cerveny, L. et al. (2006). Lack of interactions between breast cancer resistance protein (bcrp/abcg2) and selected antiepileptic agents. Epilepsia, Vol.47, No.3, (Mar), pp.461-468, ISSN 0013-9580

Chambers, T.C. et al. (1990a). Correlation of protein kinase C translocation, P-glycoprotein phosphorylation and reduced drug accumulation in multidrug resistant human KB cells. Biochemical and Biophysical Research Communications, Vol.169, No.1, (May 31), pp.253-259, ISSN 0006-291X

Chambers, T.C. et al. (1990b). Protein kinase C phosphorylates P-glycoprotein in multidrug resistant human KB carcinoma cells. Journal of Biological Chemistry, Vol.265, No.13, (May 5), pp.7679-7686, ISSN 0021-9258

Chat, M. et al. (1998). Drug metabolizing enzyme activities and superoxide formation in primary and immortalized rat brain endothelial cells. Life Sciences, Vol.62, No.2, pp.151-163, ISSN 0024-3205

Chen, Y. et al. (2009). P-glycoprotein and breast cancer resistance protein influence brain distribution of dasatinib. Journal of Pharmacology and Experimental Therapeutics, Vol.330, No.3, (Sep), pp.956-963, ISSN 1521-0103

Clarke, H.B. \& Gabrielsen, T.O. (1989). Seizure induced disruption of blood-brain barrier demonstrated by CT. Journal of Computer Assisted Tomography, Vol.13, No.5, (SepOct), pp.889-892, ISSN 0363-8715

Cobb, S. et al. (1938). Anticonvulsive action of vital dyes. Archives of Neurology and Psychiatry, Vol.40, pp.1156-1177

Cornford, E.M. et al. (1998). Interictal seizure resections show two configurations of endothelial Glut1 glucose transporter in the human blood-brain barrier. Journal of Cerebral Blood Flow and Metabolism, Vol.18, No.1, (Jan), pp.26-42, ISSN 0271-678X

Cornford, E.M. \& Oldendorf, W.H. (1986). Epilepsy and the blood-brain barrier. Advances in Neurology, Vol.44, pp.787-812, ISSN 0091-3952 
Crowe, A. \& Teoh, Y.K. (2006). Limited P-glycoprotein mediated efflux for anti-epileptic drugs. Journal of Drug Targeting, Vol.14, No.5, (Jun), pp.291-300, ISSN 1061-186X

Cucullo, L. et al. (2007). Development of a humanized in vitro blood-brain barrier model to screen for brain penetration of antiepileptic drugs. Epilepsia, Vol.48, No.3, (Mar), pp.505-516, ISSN 0013-9580

Dauchy, S. et al. (2008). ABC transporters, cytochromes P450 and their main transcription factors: expression at the human blood-brain barrier. Journal of Neurochemistry, Vol.107, No.6, (Dec), pp.1518-1528, ISSN 1471-4159

Dauchy, S. et al. (2009). Expression and transcriptional regulation of ABC transporters and cytochromes P450 in hCMEC/D3 human cerebral microvascular endothelial cells. Biochemical Pharmacology, Vol.77, No.5, (Mar 1), pp.897-909, ISSN 1873-2968

De Vries, N.A. et al. (2007). P-glycoprotein and breast cancer resistance protein: two dominant transporters working together in limiting the brain penetration of topotecan. Clinical Cancer Research, Vol.13, No.21, (Nov 1), pp.6440-6449, ISSN 10780432

Del Amo, E.M. et al. (2008). Pharmacokinetic role of L-type amino acid transporters LAT1 and LAT2. European Journal of Pharmaceutical Sciences, Vol.35, No.3, (Oct 2), pp.161174, ISSN 0928-0987

Devinsky, O. (1999). Patients with refractory seizures. New England Journal of Medicine, Vol.340, No.20, (May 20), pp.1565-1570, ISSN 0028-4793

Dixit, S.G. et al. (2005). Nitric oxide mediates increased P-glycoprotein activity in interferon\{gamma\}-stimulated human intestinal cells. Am J Physiol Gastrointest Liver Physiol, Vol.288, No.3, (Mar), pp.G533-540, ISSN 0193-1857

Dombrowski, S.M. et al. (2001). Overexpression of multiple drug resistance genes in endothelial cells from patients with refractory epilepsy. Epilepsia, Vol.42, No.12, (Dec), pp.1501-1506, ISSN 0013-9580

Dutheil, F. et al. (2009). Xenobiotic-metabolizing enzymes and transporters in the normal human brain: regional and cellular mapping as a basis for putative roles in cerebral function. Drug Metabolism and Disposition: The Biological Fate of Chemicals, Vol.37, No.7, (Jul), pp.1528-1538, ISSN 1521-009X

Dutheil, F. et al. (2010). ABC transporters and cytochromes P450 in the human central nervous system: influence on brain pharmacokinetics and contribution to neurodegenerative disorders. Expert Opin Drug Metab Toxicol, Vol.6, No.10, (Oct), pp.1161-1174, ISSN 1744-7607

Ehrlich, P. (1885). Das Sauerstoff-Bedürfniss des Organismus. Eine farbenanalytische Studie. Verlag von August Hischwald, pp.1-167

Fieschi, C. et al. (1980). Effects on EEG of the osmotic opening of the blood-brain barrier in rats. Life Sciences, Vol.27, No.3, (Jul 21), pp.239-243, ISSN 0024-3205

Filbrandt, C.R. et al. (2004). Presence and functional activity of the aryl hydrocarbon receptor in isolated murine cerebral vascular endothelial cells and astrocytes. Neurotoxicology, Vol.25, No.4, (Jun), pp.605-616, ISSN 0161-813X

Fischborn, S.V. et al. (2010). Targeting the prostaglandin E2 EP1 receptor and cyclooxygenase-2 in the amygdala kindling model in mice. Epilepsy Research, Vol.91, No.1, (Sep), pp.57-65, ISSN 1872-6844 
Friedman, A. et al. (2009). Blood-brain barrier breakdown-inducing astrocytic transformation: novel targets for the prevention of epilepsy. Epilepsy Research, Vol.85, No.2-3, (Aug), pp.142-149, ISSN 1872-6844

Geick, A. et al. (2001). Nuclear receptor response elements mediate induction of intestinal MDR1 by rifampin. Journal of Biological Chemistry, Vol.276, No.18, (May 4), pp.14581-14587, ISSN 0021-9258

Gerk, P.M. \& Vore, M. (2002). Regulation of expression of the multidrug resistanceassociated protein 2 (MRP2) and its role in drug disposition. Journal of Pharmacology and Experimental Therapeutics, Vol.302, No.2, (Aug), pp.407-415, ISSN 0022-3565

Ghersi-Egea, J.F. et al. (1994). Localization of drug-metabolizing enzyme activities to bloodbrain interfaces and circumventricular organs. Journal of Neurochemistry, Vol.62, No.3, (Mar), pp.1089-1096, ISSN 0022-3042

Ghersi-Egea, J.F. et al. (1998). Electronic spin resonance detection of superoxide and hydroxyl radicals during the reductive metabolism of drugs by rat brain preparations and isolated cerebral microvessels. Free Radical Biology and Medicine, Vol.24, No.7-8, (May), pp.1074-1081, ISSN 0891-5849

Ghersi-Egea, J.F. et al. (1988). A new aspect of the protective functions of the blood-brain barrier: activities of four drug-metabolizing enzymes in isolated rat brain microvessels. Life Sciences, Vol.42, No.24, pp.2515-2523, ISSN 0024-3205

Ghersi-Egea, J.F. et al. (1993). Subcellular localization of cytochrome P450, and activities of several enzymes responsible for drug metabolism in the human brain. Biochemical Pharmacology, Vol.45, No.3, (Feb 9), pp.647-658, ISSN 0006-2952

Ghersi-Egea, J.F. et al. (1987). Quantitative measurement of cerebral cytochrome P-450 by second derivative spectrophotometry. Journal of Neuroscience Methods, Vol.20, No.3, (Jul), pp.261-269, ISSN 0165-0270

Ghosh, C. et al. (2010). Pattern of P450 expression at the human blood-brain barrier: roles of epileptic condition and laminar flow. Epilepsia, Vol.51, No.8, (Aug), pp.1408-1417, ISSN 1528-1167

Ghosh, C. et al. (2011). Cellular localization and functional significance of CYP3A4 in the human epileptic brain. Epilepsia, Vol.52, No.3, (Mar), pp.562-571, ISSN 1528-1167

Goldmann, E.E. (1909). Die äussere und innere Sekretion des gesunden und kranken Organismus im Lichte der "vitalen Färbung". Beiträge zur klinischen Chirurgie, Vol.64, pp.192-265

Goldmann, E.E. (1913) Vitalfärbung am Zentralnervensystem. Beitrag zur Physio-Pathologie des Plexus Chorioideus und der Hirnhäute. Verlag der königlichen Akademie der Wissenschaften, Berlin.

Goldstein, G.W. \& Betz, A.L. (1983). Recent advances in understanding brain capillary function. Annals of Neurology, Vol.14, No.4, (Oct), pp.389-395

Goralski, K.B. et al. (2003). Downregulation of mdr1a expression in the brain and liver during CNS inflammation alters the in vivo disposition of digoxin. British Journal of Pharmacology, Vol.139, No.1, (May), pp.35-48, ISSN 0007-1188

Hartz, A.M. et al. (2004). Rapid regulation of P-glycoprotein at the blood-brain barrier by endothelin-1. Molecular Pharmacology, Vol.66, No.3, (Sep), pp.387-394, ISSN 0026$895 X$ 
Hartz, A.M. et al. (2010). Restoring blood-brain barrier P-glycoprotein reduces brain amyloid-beta in a mouse model of Alzheimer's disease. Molecular Pharmacology, Vol.77, No.5, (May), pp.715-723, ISSN 1521-0111

Hauser, W.A. \& Lee, J.R. (2002). Do seizures beget seizures? Progress in Brain Research, Vol.135, pp.215-219, ISSN 0079-6123

Hayashi, K. et al. (2006). HIV-TAT protein upregulates expression of multidrug resistance protein 1 in the blood-brain barrier. Journal of Cerebral Blood Flow and Metabolism, Vol.26, No.8, (Aug), pp.1052-1065, ISSN 0271-678X

Ho, E.A. \& Piquette-Miller, M. (2006). Regulation of multidrug resistance by proinflammatory cytokines. Curr Cancer Drug Targets, Vol.6, No.4, (Jun), pp.295-311, ISSN 1568-0096

Hoffmann, K. et al. (2006). Expression of the multidrug transporter MRP2 in the blood-brain barrier after pilocarpine-induced seizures in rats. Epilepsy Research, Vol.69, No.1, (Apr), pp.1-14, ISSN 0920-1211

Holtman, L. et al. (2010). Cox-2 inhibition can lead to adverse effects in a rat model for temporal lobe epilepsy. Epilepsy Research, Vol.91, No.1, (Sep), pp.49-56, ISSN 18726844

Horowitz, S.W. et al. (1992). Complex partial seizure-induced transient MR enhancement. Journal of Computer Assisted Tomography, Vol.16, No.5, (Sep-Oct), pp.814-816, ISSN 0363-8715

Hrycay, E.G. \& Bandiera, S.M. (2009). Expression, function and regulation of mouse cytochrome P450 enzymes: comparison with human P450 enzymes. Curr Drug Metab, Vol.10, No.10, (Dec), pp.1151-1183, ISSN 1875-5453

Huber, J.D. et al. (2001). Molecular physiology and pathophysiology of tight junctions in the blood-brain barrier. Trends in Neurosciences, Vol.24, No.12, (Dec), pp.719-725, ISSN

Iannetti, P. et al. (2005). Calcium-channel blocker verapamil administration in prolonged and refractory status epilepticus. Epilepsia, Vol.46, No.6, (Jun), pp.967-969, ISSN 0013-9580

Ito, K. et al. (2011). Quantitative membrane protein expression at the blood-brain barrier of adult and younger cynomolgus monkeys. Journal of Pharmaceutical Sciences, (Jan 19), pp., ISSN 1520-6017

Ivens, S. et al. (2010). Blood-brain barrier breakdown as a novel mechanism underlying cerebral hyperperfusion syndrome. Journal of Neurology, Vol.257, No.4, (Apr), pp.615-620, ISSN 1432-1459

Jambroszyk, M. et al. (2011). Add-on treatment with verapamil in pharmacoresistant canine epilepsy. Epilepsia, Vol.52, No.2, (Feb), pp.284-291, ISSN 1528-1167

Jedlitschky, G. et al. (1996). Transport of glutathione, glucuronate, and sulfate conjugates by the MRP gene-encoded conjugate export pump. Cancer Research, Vol.56, No.5, (Mar 1), pp.988-994, ISSN 0008-5472

Johansson, B.B. (1981). Indomethacin and cerebrovascular permeability to albumin in acute hypertension and cerebral embolism in the rat. Experimental Brain Research, Vol.42, No.3-4, pp.331-336, ISSN 0014-4819

Kalalinia, F. et al. (2011). Potential role of cyclooxygenase-2 on the regulation of the drug efflux transporter ABCG2 in breast cancer cell lines. Journal of Cancer Research and Clinical Oncology, Vol.137, No.2, (Feb), pp.321-330, ISSN 1432-1335 
Kamiie, J. et al. (2008). Quantitative atlas of membrane transporter proteins: development and application of a highly sensitive simultaneous LC/MS/MS method combined with novel in-silico peptide selection criteria. Pharmaceutical Research, Vol.25, No.6, (Jun), pp.1469-1483, ISSN 0724-8741

Kast, H.R. et al. (2002). Regulation of multidrug resistance-associated protein 2 (ABCC2) by the nuclear receptors pregnane $X$ receptor, farnesoid $X$-activated receptor, and constitutive androstane receptor. Journal of Biological Chemistry, Vol.277, No.4, (Jan 25), pp.2908-2915, ISSN 0021-9258

Kim, H.G. et al. (2011). Metformin inhibits P-glycoprotein expression via the NF-kappaB pathway and CRE transcriptional activity through AMPK activation. British Journal of Pharmacology, Vol.162, No.5, (Mar), pp.1096-1108, ISSN 1476-5381

Kitano, T. et al. (2002). Polarized glucose transporters and mRNA expression properties in newly developed rat syncytiotrophoblast cell lines, TR-TBTs. Journal of Cellular Physiology, Vol.193, No.2, (Nov), pp.208-218, ISSN 0021-9541

Klotz, U. (2007). The role of pharmacogenetics in the metabolism of antiepileptic drugs: pharmacokinetic and therapeutic implications. Clinical Pharmacokinetics, Vol.46, No.4, pp.271-279, ISSN 0312-5963

Kniesel, U. \& Wolburg, H. (2000). Tight junctions of the blood-brain barrier. Cellular and Molecular Neurobiology, Vol.20, No.1, (Feb), pp.57-76

Kubota, H. et al. (2006). Distribution and functional activity of P-glycoprotein and multidrug resistance-associated proteins in human brain microvascular endothelial cells in hippocampal sclerosis. Epilepsy Research, Vol.68, No.3, (Mar), pp.213-228, ISSN 0920-1211

Kuteykin-Teplyakov, K. et al. (2009). Complex time-dependent alterations in the brain expression of different drug efflux transporter genes after status epilepticus. Epilepsia, Vol.50, No.4, (Apr), pp.887-897, ISSN 1528-1167

Kwan, P. \& Brodie, M.J. (2003). Clinical trials of antiepileptic medications in newly diagnosed patients with epilepsy. Neurology, Vol.60, No.11 Suppl 4, (Jun 10), pp.S212, ISSN 1526-632X

Lazarowski, A. et al. (2007). ABC transporters during epilepsy and mechanisms underlying multidrug resistance in refractory epilepsy. Epilepsia, Vol.48 Suppl 5, pp.140-149, ISSN 0013-9580

Lazarowski, A. et al. (1999). Tuberous sclerosis associated with MDR1 gene expression and drug-resistant epilepsy. Pediatric Neurology, Vol.21, No.4, (Oct), pp.731-734, ISSN 0887-8994 (Print)

Lee, J.C. \& Olszewski, J. (1961). Increased cerebrovascular permeability after repeated electroshocks. Neurology, Vol.11, (Jun), pp.515-519, ISSN 0028-3878

Leslie, E.M. et al. (2004). Arsenic transport by the human multidrug resistance protein 1 (MRP1/ABCC1). Evidence that a tri-glutathione conjugate is required. Journal of Biological Chemistry, Vol.279, No.31, (Jul 30), pp.32700-32708, ISSN 0021-9258

Lewandowsky, M. (1900). Zur Lehre von der Cerebrospinalflüssigkeit. Zeitschrift fur Klinische Medizin, Vol.40, pp.480-494

Lin, C.J. et al. (2010). Cellular localization of the organic cation transporters, OCT1 and OCT2, in brain microvessel endothelial cells and its implication for MPTP transport 
across the blood-brain barrier and MPTP-induced dopaminergic toxicity in rodents. Journal of Neurochemistry, Vol.114, No.3, (Aug), pp.717-727, ISSN 1471-4159

Liu, X. et al. (2008). Progress in brain penetration evaluation in drug discovery and development. Journal of Pharmacology and Experimental Therapeutics, Vol.325, No.2, (May), pp.349-356, ISSN 1521-0103

Liu, X. et al. (2007). Increased P-glycoprotein expression and decreased phenobarbital distribution in the brain of pentylenetetrazole-kindled rats. Neuropharmacology, Vol.53, No.5, (Oct), pp.657-663, ISSN 0028-3908

Loe, D.W. et al. (1996). Multidrug resistance protein (MRP)-mediated transport of leukotriene $\mathrm{C} 4$ and chemotherapeutic agents in membrane vesicles. Demonstration of glutathione-dependent vincristine transport. Journal of Biological Chemistry, Vol.271, No.16, (Apr 19), pp.9675-9682, ISSN 0021-9258

Loscher, W. \& Potschka, H. (2005). Drug resistance in brain diseases and the role of drug efflux transporters. Nat Rev Neurosci, Vol.6, No.8, (Aug), pp.591-602, ISSN 1471$003 X$

Luo, G. et al. (2002). CYP3A4 induction by drugs: correlation between a pregnane $\mathrm{X}$ receptor reporter gene assay and CYP3A4 expression in human hepatocytes. Drug Metabolism and Disposition: The Biological Fate of Chemicals, Vol.30, No.7, (Jul), pp.795-804, ISSN 0090-9556

Maines, L.W. et al. (2005). Evaluation of the role of P-glycoprotein in the uptake of paroxetine, clozapine, phenytoin and carbamazapine by bovine retinal endothelial cells. Neuropharmacology, Vol.49, No.5, (Oct), pp.610-617, ISSN 0028-3908

Marchi, N. et al. (2007). Seizure-promoting effect of blood-brain barrier disruption. Epilepsia, Vol.48, No.4, (Apr), pp.732-742, ISSN 0013-9580

Marchi, N. et al. (2006). Determinants of drug brain uptake in a rat model of seizureassociated malformations of cortical development. Neurobiology of Disease, Vol.24, No.3, (Dec), pp.429-442, ISSN 0969-9961

Marchi, N. et al. (2005). A pilot study on brain-to-plasma partition of 10,11-dyhydro-10hydroxy-5H-dibenzo(b,f)azepine-5-carboxamide and MDR1 brain expression in epilepsy patients not responding to oxcarbazepine. Epilepsia, Vol.46, No.10, (Oct), pp.1613-1619, ISSN 0013-9580

Marchi, N. et al. (2004). Significance of MDR1 and multiple drug resistance in refractory human epileptic brain. BMC Med, Vol.2, (Oct 9), pp.37, ISSN 1741-7015

Martin, C. et al. (1999). The molecular interaction of the high affinity reversal agent XR9576 with P-glycoprotein. British Journal of Pharmacology, Vol.128, No.2, (Sep), pp.403411, ISSN 0007-1188

Matter, K. \& Balda, M.S. (2003a). Holey barrier: claudins and the regulation of brain endothelial permeability. Journal of Cell Biology, Vol.161, No.3, (May 12), pp.459-460, ISSN 0021-9525

Matter, K. \& Balda, M.S. (2003b). Signalling to and from tight junctions. Nat Rev Mol Cell Biol, Vol.4, No.3, (Mar), pp.225-236

Michaluk, P. \& Kaczmarek, L. (2007). Matrix metalloproteinase-9 in glutamate-dependent adult brain function and dysfunction. Cell Death and Differentiation, Vol.14, No.7, (Jul), pp.1255-1258, ISSN 1350-9047 
Mihaly, A. \& Bozoky, B. (1984). Immunohistochemical localization of extravasated serum albumin in the hippocampus of human subjects with partial and generalized epilepsies and epileptiform convulsions. Acta Neuropathol, Vol.65, No.1, pp.25-34, ISSN 0001-6322

Miksys, S. \& Tyndale, R.F. (2004). The unique regulation of brain cytochrome P450 2 (CYP2) family enzymes by drugs and genetics. Drug Metabolism Reviews, Vol.36, No.2, (May), pp.313-333, ISSN 0360-2532

Miller, D.S. et al. (2008). Modulation of P-glycoprotein at the blood-brain barrier: opportunities to improve central nervous system pharmacotherapy. Pharmacological Reviews, Vol.60, No.2, (Jun), pp.196-209, ISSN 1521-0081

Miller, D.S. et al. (1998). Protein kinase C regulation of p-glycoprotein-mediated xenobiotic secretion in renal proximal tubule. American Journal of Physiology, Vol.275, No.5 Pt 2, (Nov), pp.F785-795, ISSN 0002-9513

Minn, A. et al. (1991). Drug metabolizing enzymes in the brain and cerebral microvessels. Brain Research Brain Research Reviews, Vol.16, No.1, (Jan-Apr), pp.65-82

Mistry, P. et al. (2001). In vitro and in vivo reversal of P-glycoprotein-mediated multidrug resistance by a novel potent modulator, XR9576. Cancer Research, Vol.61, No.2, (Jan 15), pp.749-758, ISSN 0008-5472

Mukherjee, S.C. (2001). Epileptic and non-epileptic seizures. Journal of the Indian Medical Association, Vol.99, No.2, (Feb), pp.78-79, ISSN 0019-5847

Nag, S. (2003). Morphology and molecular properties of cellular components of normal cerebral vessels. Methods Mol Med, Vol.89, pp.3-36

Nagy, Z. et al. (1984). Fracture faces of cell junctions in cerebral endothelium during normal and hyperosmotic conditions. Laboratory Investigation, Vol.50, No.3, (Mar), pp.313322

Narang, V.S. et al. (2008). Dexamethasone increases expression and activity of multidrug resistance transporters at the rat blood-brain barrier. Am J Physiol Cell Physiol, Vol.295, No.2, (Aug), pp.C440-450, ISSN 0363-6143

Nawa, A. et al. (2010). Inducible nitric oxide synthase-mediated decrease of intestinal Pglycoprotein expression under streptozotocin-induced diabetic conditions. Life Sciences, Vol.86, No.11-12, (Mar 13), pp.402-409, ISSN 1879-0631

Ndode-Ekane, X.E. et al. (2010). Vascular changes in epilepsy: functional consequences and association with network plasticity in pilocarpine-induced experimental epilepsy. Neuroscience, Vol.166, No.1, (Mar 10), pp.312-332, ISSN 1873-7544

Nemeroff, C.B. \& Crisley, F.D. (1975). Monosodium L-glutamate-induced convulsions: temporary alteration in blood-brain barrier permeability to plasma proteins. Environmental Physiology and Biochemistry, Vol.5, No.6, pp.389-395, ISSN 0300-5429

Neuwelt, E.A. et al. (2011). Engaging neuroscience to advance translational research in brain barrier biology. Nat Rev Neurosci, Vol.12, No.3, (Mar), pp.169-182, ISSN 1471-0048

Nishijima, T. et al. (2010). Neuronal activity drives localized blood-brain-barrier transport of serum insulin-like growth factor-I into the CNS. Neuron, Vol.67, No.5, (Sep 9), pp.834-846, ISSN 1097-4199

Nishimura, M. et al. (2004). Tissue-specific mRNA expression profiles of human nuclear receptor subfamilies. Drug Metab Pharmacokinet, Vol.19, No.2, (Apr), pp.135-149, ISSN 1347-4367 
Nitsch, C. \& Hubauer, H. (1986). Distant blood-brain barrier opening in subfields of the rat hippocampus after intrastriatal injections of kainic acid but not ibotenic acid. Neuroscience Letters, Vol.64, No.1, (Feb 14), pp.53-58, ISSN 0304-3940

Nitsch, C. \& Klatzo, I. (1983). Regional patterns of blood-brain barrier breakdown during epileptiform seizures induced by various convulsive agents. Journal of the Neurological Sciences, Vol.59, No.3, (Jun), pp.305-322, ISSN 0022-510X

O'kane, R.L. \& Hawkins, R.A. (2003). Na+-dependent transport of large neutral amino acids occurs at the abluminal membrane of the blood-brain barrier. Am J Physiol Endocrinol Metab, Vol.285, No.6, (Dec), pp.E1167-1173, ISSN 0193-1849

O'kane, R.L. et al. (1999). $\mathrm{Na}(+)$-dependent glutamate transporters (EAAT1, EAAT2, and EAAT3) of the blood-brain barrier. A mechanism for glutamate removal. Journal of Biological Chemistry, Vol.274, No.45, (Nov 5), pp.31891-31895, ISSN 0021-9258

Oby, E. \& Janigro, D. (2006). The blood-brain barrier and epilepsy. Epilepsia, Vol.47, No.11, (Nov), pp.1761-1774, ISSN 0013-9580

Oude Elferink, R.P. \& Jansen, P.L. (1994). The role of the canalicular multispecific organic anion transporter in the disposal of endo- and xenobiotics. Pharmacology and Therapeutics, Vol.64, No.1, (Oct), pp.77-97, ISSN 0163-7258

Owen, A. et al. (2001). Carbamazepine is not a substrate for P-glycoprotein. British Journal of Clinical Pharmacology, Vol.51, No.4, (Apr), pp.345-349, ISSN 0306-5251

Oztas, B. \& Kaya, M. (1991). The effect of acute hypertension on blood-brain barrier permeability to albumin during experimentally induced epileptic seizures. Pharmacological Research, Vol.23, No.1, (Jan), pp.41-46, ISSN 1043-6618

Oztas, B. \& Sandalci, U. (1984). Reversibility of blood-brain barrier dysfunction in acute hypertension induced by angiotensin. Experimental Neurology, Vol.84, No.3, (Jun), pp.666-670, ISSN 0014-4886

Padou, V. et al. (1995). Changes in transport of [14C] alpha-aminoisobutyric acid across the blood-brain barrier during pentylenetetrazol-induced status epilepticus in the immature rat. Epilepsy Research, Vol.22, No.3, (Nov), pp.175-183, ISSN 0920-1211

Palade, G.E. (1961). Blood capillaries of the heart and other organs. Circulation, Vol.24, (Aug), pp.368-388, ISSN 0009-7322

Pardridge, W.M. (1991). Blood-brain barrier transport of glucose, free fatty acids, and ketone bodies. Advances in Experimental Medicine and Biology, Vol.291, pp.43-53, ISSN 00652598

Pardridge, W.M. (2003a). Blood-brain barrier drug targeting: the future of brain drug development. Mol Interv, Vol.3, No.2, (Mar), pp.90-105, 151, ISSN 1534-0384

Pardridge, W.M. (2003b). Blood-brain barrier genomics and the use of endogenous transporters to cause drug penetration into the brain. Curr Opin Drug Discov Devel, Vol.6, No.5, (Sep), pp.683-691, ISSN 1367-6733

Patel, V.A. et al. (2002). Regulation of MDR-1 (P-glycoprotein) by cyclooxygenase-2. Journal of Biological Chemistry, Vol.277, No.41, (Oct 11), pp.38915-38920, ISSN 0021-9258

Pekcec, A. et al. (2009). Targeting prostaglandin E2 EP1 receptors prevents seizureassociated P-glycoprotein up-regulation. Journal of Pharmacology and Experimental Therapeutics, Vol.330, No.3, (Sep), pp.939-947, ISSN 1521-0103 
Pennock, G.D. et al. (1991). Systemic toxic effects associated with high-dose verapamil infusion and chemotherapy administration. Journal of the National Cancer Institute, Vol.83, No.2, (Jan 16), pp.105-110, ISSN 0027-8874

Petito, C.K. et al. (1977). Ultrastructural characteristics of the brain and blood-brain barrier in experimental seizures. Brain Research, Vol.127, No.2, (May 27), pp.251-267, ISSN 0006-8993

Poller, B. et al. (2010). Regulation of BCRP (ABCG2) and P-glycoprotein (ABCB1) by cytokines in a model of the human blood-brain barrier. Cellular and Molecular Neurobiology, Vol.30, No.1, (Jan), pp.63-70, ISSN 1573-6830

Potschka, H. et al. (2002). P-Glycoprotein-mediated efflux of phenobarbital, lamotrigine, and felbamate at the blood-brain barrier: evidence from microdialysis experiments in rats. Neuroscience Letters, Vol.327, No.3, (Jul 26), pp.173-176, ISSN 0304-3940

Potschka, H. \& Loscher, W. (2001). In vivo evidence for P-glycoprotein-mediated transport of phenytoin at the blood-brain barrier of rats. Epilepsia, Vol.42, No.10, (Oct), pp.1231-1240, ISSN 0013-9580

Potschka, H. et al. (2004). Pharmacoresistance and expression of multidrug transporter Pglycoprotein in kindled rats. Neuroreport, Vol.15, No.10, (Jul 19), pp.1657-1661, ISSN 0959-4965

Ravindranath, V. et al. (1990). NADPH cytochrome P-450 reductase in rat, mouse and human brain. Biochemical Pharmacology, Vol.39, No.6, (Mar 15), pp.1013-1018, ISSN 0006-2952

Reese, T.S. \& Karnovsky, M.J. (1967). Fine structural localization of a blood-brain barrier to exogenous peroxidase. Journal of Cell Biology, Vol.34, No.1, (Jul), pp.207-217

Rieder, C.R. et al. (2000). Human brain cytochrome P450 1B1: immunohistochemical localization in human temporal lobe and induction by dimethylbenz(a)anthracene in astrocytoma cell line (MOG-G-CCM). Neuroscience Letters, Vol.278, No.3, (Jan 14), pp.177-180, ISSN 0304-3940

Rigau, V. et al. (2007). Angiogenesis is associated with blood-brain barrier permeability in temporal lobe epilepsy. Brain, Vol.130, No.Pt 7, (Jul), pp.1942-1956, ISSN 1460-2156

Rigor, R.R. et al. (2010). Activation of PKC isoform beta(I) at the blood-brain barrier rapidly decreases P-glycoprotein activity and enhances drug delivery to the brain. Journal of Cerebral Blood Flow and Metabolism, Vol.30, No.7, (Jul), pp.1373-1383, ISSN 1559-7016

Rizzi, M. et al. (2002). Limbic seizures induce P-glycoprotein in rodent brain: functional implications for pharmacoresistance. Journal of Neuroscience, Vol.22, No.14, (Jul 15), pp.5833-5839, ISSN 1529-2401

Rodriguez-Baeza, A. et al. (2003). Morphological features in human cortical brain microvessels after head injury: a three-dimensional and immunocytochemical study. Anat Rec A Discov Mol Cell Evol Biol, Vol.273, No.1, (Jul), pp.583-593

Ronne-Engstrom, E. et al. (1992). Intracerebral microdialysis of extracellular amino acids in the human epileptic focus. Journal of Cerebral Blood Flow and Metabolism, Vol.12, No.5, (Sep), pp.873-876, ISSN 0271-678X

Salazar, A.M. et al. (1985). Epilepsy after penetrating head injury. I. Clinical correlates: a report of the Vietnam Head Injury Study. Neurology, Vol.35, No.10, (Oct), pp.14061414, ISSN 0028-3878 
Schaefer, J.A. et al. (1975). Disturbance of the blood-brain barrier in electroshock-induced seizures. Neurology, Vol.25, pp.382

Schlichtiger, J. et al. (2010). Celecoxib treatment restores pharmacosensitivity in a rat model of pharmacoresistant epilepsy. British Journal of Pharmacology, Vol.160, No.5, (Jul), pp.1062-1071, ISSN 1476-5381

Seiffert, E. et al. (2004). Lasting blood-brain barrier disruption induces epileptic focus in the rat somatosensory cortex. Journal of Neuroscience, Vol.24, No.36, (Sep 8), pp.78297836, ISSN 1529-2401

Shang, W. et al. (2008). Expressions of glutathione S-transferase alpha, mu, and pi in brains of medically intractable epileptic patients. BMC Neurosci, Vol.9, pp.67, ISSN 14712202

Simpson, I.A. et al. (2007). Supply and demand in cerebral energy metabolism: the role of nutrient transporters. Journal of Cerebral Blood Flow and Metabolism, Vol.27, No.11, (Nov), pp.1766-1791, ISSN 0271-678X

Singh, A. et al. (2010). Expression of ABCG2 (BCRP) is regulated by Nrf2 in cancer cells that confers side population and chemoresistance phenotype. Mol Cancer Ther, Vol.9, No.8, (Aug), pp.2365-2376, ISSN 1538-8514

Sisodiya, S.M. et al. (2002). Drug resistance in epilepsy: expression of drug resistance proteins in common causes of refractory epilepsy. Brain, Vol.125, No.Pt 1, (Jan), pp.22-31, ISSN 0006-8950

Sisodiya, S.M. et al. (2003). Major vault protein, a marker of drug resistance, is upregulated in refractory epilepsy. Epilepsia, Vol.44, No.11, (Nov), pp.1388-1396, ISSN 0013-9580

Sisodiya, S.M. et al. (2006). Vascular colocalization of P-glycoprotein, multidrug-resistance associated protein 1, breast cancer resistance protein and major vault protein in human epileptogenic pathologies. Neuropathology and Applied Neurobiology, Vol.32, No.1, (Feb), pp.51-63, ISSN 0305-1846

Sokrab, T.E. et al. (1989). Endogenous serum albumin content in brain after short-lasting epileptic seizures. Brain Research, Vol.489, No.2, (Jun 12), pp.231-236, ISSN 00068993

Spatz, H. (1933). Die Bedeutung der vitalen Färbung für die Lehre vom Stoffaustausch zwischen dem Zentralnervensystem und dem übrigen Körper. Archiv für Phsychiatrie, Vol.101, pp.267-358

Sperling, M.R. et al. (1999). Seizure control and mortality in epilepsy. Annals of Neurology, Vol.46, No.1, (Jul), pp.45-50, ISSN 0364-5134

Stamatovic, S.M. et al. (2008). Brain endothelial cell-cell junctions: how to "open" the blood brain barrier. Curr Neuropharmacol, Vol.6, No.3, (Sep), pp.179-192, ISSN 1570-159X

Stollberger, C. \& Finsterer, J. (2003). Nonsteroidal anti-inflammatory drugs in patients with cardio- or cerebrovascular disorders. Zeitschrift fur Kardiologie, Vol.92, No.9, (Sep), pp.721-729, ISSN 0300-5860

Strazielle, N. \& Ghersi-Egea, J.F. (1999). Demonstration of a coupled metabolism-efflux process at the choroid plexus as a mechanism of brain protection toward xenobiotics. Journal of Neuroscience, Vol.19, No.15, (Aug 1), pp.6275-6289, ISSN 02706474

Su, T.Z. et al. (1995). Transport of gabapentin, a gamma-amino acid drug, by system 1 alphaamino acid transporters: a comparative study in astrocytes, synaptosomes, and 
CHO cells. Journal of Neurochemistry, Vol.64, No.5, (May), pp.2125-2131, ISSN 00223042

Sukhai, M. et al. (2001). Decreased expression of P-glycoprotein in interleukin-1beta and interleukin-6 treated rat hepatocytes. Inflammation Research, Vol.50, No.7, (Jul), pp.362-370, ISSN 1023-3830

Summers, M.A. et al. (2004). Use of verapamil as a potential P-glycoprotein inhibitor in a patient with refractory epilepsy. Annals of Pharmacotherapy, Vol.38, No.10, (Oct), pp.1631-1634, ISSN 1060-0280

Takara, K. et al. (2004). Carvedilol: a new candidate for reversal of MDR1/P-glycoproteinmediated multidrug resistance. Anti-Cancer Drugs, Vol.15, No.4, (Apr), pp.303-309, ISSN 0959-4973

Tan, K.P. et al. (2010). Aryl hydrocarbon receptor is a transcriptional activator of the human breast cancer resistance protein (BCRP/ABCG2). Molecular Pharmacology, Vol.78, No.2, (Aug), pp.175-185, ISSN 1521-0111

Tayarani, I. et al. (1987). Evidence for an alanine, serine, and cysteine system of transport in isolated brain capillaries. Journal of Cerebral Blood Flow and Metabolism, Vol.7, No.5, (Oct), pp.585-591, ISSN 0271-678X

Thevenod, F. et al. (2000). Up-regulation of multidrug resistance P-glycoprotein via nuclear factor-kappaB activation protects kidney proximal tubule cells from cadmium- and reactive oxygen species-induced apoptosis. Journal of Biological Chemistry, Vol.275, No.3, (Jan 21), pp.1887-1896, ISSN 0021-9258

Tishler, D.M. et al. (1995). MDR1 gene expression in brain of patients with medically intractable epilepsy. Epilepsia, Vol.36, No.1, (Jan), pp.1-6, ISSN 0013-9580

Tomkins, O. et al. (2011). Blood-brain barrier breakdown following traumatic brain injury: a possible role in posttraumatic epilepsy. Cardiovasc Psychiatry Neurol, Vol.2011, pp.765923, ISSN 2090-0171

Tomkins, O. et al. (2008). Blood-brain barrier disruption in post-traumatic epilepsy. Journal of Neurology, Neurosurgery and Psychiatry, Vol.79, No.7, (Jul), pp.774-777, ISSN 1468$330 \mathrm{X}$

Ueda, K. et al. (2007). Glutathione S-transferase M1 null genotype as a risk factor for carbamazepine-induced mild hepatotoxicity. Pharmacogenomics, Vol.8, No.5, (May), pp.435-442, ISSN 1744-8042

Ueda, Y. \& Tsuru, N. (1995). Simultaneous monitoring of the seizure-related changes in extracellular glutamate and gamma-aminobutyric acid concentration in bilateral hippocampi following development of amygdaloid kindling. Epilepsy Research, Vol.20, No.3, (Mar), pp.213-219, ISSN 0920-1211

Van Vliet, E.A. et al. (2005). Expression of multidrug transporters MRP1, MRP2, and BCRP shortly after status epilepticus, during the latent period, and in chronic epileptic rats. Epilepsia, Vol.46, No.10, (Oct), pp.1569-1580, ISSN 0013-9580

Van Vliet, E.A. et al. (2006). Inhibition of the multidrug transporter P-glycoprotein improves seizure control in phenytoin-treated chronic epileptic rats. Epilepsia, Vol.47, No.4, (Apr), pp.672-680, ISSN 0013-9580

Van Vliet, E.A. et al. (2007). Region-specific overexpression of P-glycoprotein at the bloodbrain barrier affects brain uptake of phenytoin in epileptic rats. Journal of 
Pharmacology and Experimental Therapeutics, Vol.322, No.1, (Jul), pp.141-147, ISSN 0022-3565

Van Vliet, E.A. et al. (2010). COX-2 inhibition controls P-glycoprotein expression and promotes brain delivery of phenytoin in chronic epileptic rats. Neuropharmacology, Vol.58, No.2, (Feb), pp.404-412, ISSN 1873-7064

Vogelgesang, S. et al. (2004). Expression of multidrug transporters in dysembryoplastic neuroepithelial tumors causing intractable epilepsy. Clinical Neuropathology, Vol.23, No.5, (Sep-Oct), pp.223-231, ISSN 0722-5091

Volk, B. et al. (1988). First evidence of cytochrome P-450 induction in the mouse brain by phenytoin. Neuroscience Letters, Vol.84, No.2, (Jan 22), pp.219-224, ISSN 0304-3940

Volk, B. et al. (1995). Localization and characterization of cytochrome P450 in the brain. In vivo and in vitro investigations on phenytoin- and phenobarbital-inducible isoforms. Toxicology Letters, Vol.82-83, (Dec), pp.655-662, ISSN 0378-4274

Von Wedel-Parlow, M. et al. (2009). Regulation of major efflux transporters under inflammatory conditions at the blood-brain barrier in vitro. Journal of Neurochemistry, Vol.111, No.1, (Oct), pp.111-118, ISSN 1471-4159

Vorbrodt, A.W. \& Dobrogowska, D.H. (2003). Molecular anatomy of intercellular junctions in brain endothelial and epithelial barriers: electron microscopist's view. Brain Research Brain Research Reviews, Vol.42, No.3, (Jun), pp.221-242

Walther, B. et al. (1986). Subcellular distribution of cytochrome P-450 in the brain. Brain Research, Vol.375, No.2, (Jun 11), pp.338-344, ISSN 0006-8993

Wang, X. et al. (2011). Aryl hydrocarbon receptor-mediated up-regulation of ATP-driven xenobiotic efflux transporters at the blood-brain barrier. FASEB Journal, Vol.25, No.2, (Feb), pp.644-652, ISSN 1530-6860

Wang, X. et al. (2010). Constitutive androstane receptor-mediated up-regulation of ATPdriven xenobiotic efflux transporters at the blood-brain barrier. Molecular Pharmacology, Vol.78, No.3, (Sep 1), pp.376-383, ISSN 1521-0111

Weiss, J. et al. (2003). Interaction of antiepileptic drugs with human P-glycoprotein in vitro. Journal of Pharmacology and Experimental Therapeutics, Vol.307, No.1, (Oct), pp.262267, ISSN 0022-3565

Westergaard, E. (1980). Ultrastructural permeability properties of cerebral microvasculature under normal and experimental conditions after application of tracers. Advances in Neurology, Vol.28, pp.55-74, ISSN 0091-3952

Westergaard, E. et al. (1978). Increased permeability to horseradish peroxidase across cerebral vessels, evoked by electrically induced seizures in the rat. Acta Neuropathol, Vol.41, No.1, (Jan 19), pp.73-80, ISSN 0001-6322

Wolburg, H. \& Lippoldt, A. (2002). Tight junctions of the blood-brain barrier: development, composition and regulation. Vascul Pharmacol, Vol.38, No.6, (Jun), pp.323-337, ISSN

$\mathrm{Yu}, \mathrm{C}$. et al. (2008). Neuroinflammation activates Mdr1b efflux transport through NFkappaB: promoter analysis in BBB endothelia. Cellular Physiology and Biochemistry, Vol.22, No.5-6, pp.745-756, ISSN 1421-9778

Zhu, H.J. \& Liu, G.Q. (2004). Glutamate up-regulates P-glycoprotein expression in rat brain microvessel endothelial cells by an NMDA receptor-mediated mechanism. Life Sciences, Vol.75, No.11, (Jul 30), pp.1313-1322, ISSN 0024-3205 
Zibell, G. et al. (2009). Prevention of seizure-induced up-regulation of endothelial Pglycoprotein by COX-2 inhibition. Neuropharmacology, Vol.Epub ahead of print, doi:10.1016/j.neuropharm.2009.01.009, pp., ISSN

Zlokovic, B.V. \& Apuzzo, M.L. (1998). Strategies to circumvent vascular barriers of the central nervous system. Neurosurgery, Vol.43, No.4, (Oct), pp.877-878 


\title{
New Tools for Understanding Epilepsy
}

\author{
Fatima Shad Kaneez ${ }^{1}$ and Faisal Khan ${ }^{2}$ \\ ${ }^{1}$ PAP RSB Institute of Health Sciences Universiti Brunei Darussalam, \\ Jalan Tungku Link, Gadong \\ ${ }^{2}$ Dr. Panjwani Center for Molecular Medicine and Drug research, \\ International Center for Chemical and Biological Science, University of Karachi, Karachi \\ ${ }^{1}$ Brunei Darussalam \\ 2Pakistan
}

\section{Introduction}

Epilepsy is one of the most common serious chronic neurological disorders, which is characterized by recurrent seizures with a prevalence rate of 3-6 per 1000 worldwide (Begley et al., 2007). Epilepsy is not a form of mental illness and is not an indicator of intelligence. Epilepsy affects people of any origin or race with high and low intelligence. Some people with mental retardation may have epilepsy, but most people with epilepsy are not mentally retarded (Avoli M et.al, 2001). There are many types of epilepsies and many factors such as neurotransmitters, transporters, granule cells, voltage gated ionic currents and non-neural proliferation may act as major players for this disorder (Berkovic S.F., 1998; Zaid Afawi et al, 2010). According to the literature and our work, both ligand and voltage gated ion channels in neurons and platelets seem to work together during the onset of epilepsy (Fatima Shad K (un published data); Kocsis, J.D. and Mattson, R.H., 1996). We also found similarities in the receptor's profiles of neurons and platelets mainly for serotonin, GABA and glutamate (Fatima Shad K, 2006; Fatima Shad K and Saeed SA, 2009, 2007). Recently, there has been growing evidence that serotonergic neurotransmission modulates experimentally induced seizures and is involved in the enhanced seizure susceptibility observed in some genetically epilepsy-prone animals (Methvin Isaac, 2005). Scientists are now focusing on targets other than brain receptors for the development of anti-epileptic drugs. The brain is an extraordinarily complex organ; there are several concepts about the brain which needed to be cleared when it comes to the understanding of epilepsy (Berkovic S.F., 1998). Firstly, the brain works on electricity and continuously generates tiny electrical impulses in an orderly pattern. Secondly, loss of nerve cells may contribute to the development of epilepsy in some cases. For example, prolonged lack of oxygen may cause a selective loss of cells in the hippocampus, which may lead to epilepsy. According to one theory, epilepsy is caused by an imbalance between excitatory and inhibitory neurotransmitters. If the inhibitory neurotransmitters in the brain are not active enough, or if the excitatory ones are too active, then it's more likely to have seizures. Many of the new medicines being developed to treat epilepsy try to influence these neurotransmitters, by increasing inhibitory and reducing excitatory neurotransmitter's activities. Either way, the idea is to have less uncontrolled electrical activity in the brain, and therefore fewer seizures 
(Steinlein, O. K. \& Noebels, J. L., 2000). Another concept important in relation with epilepsy is that different areas of the brain control different functions. If seizures arise from a specific area of the brain, then the initial symptoms of the seizure often reflect the functions of that area. The right half of the brain controls the left side of the body, and the left half of the brain controls the right side of the body. So if a seizure starts from the right side of the brain, in the area that controls movement in the thumb, then the attack may begin with jerking of the left thumb or hand. Thus a seizure occurs when the brain's nerve cells misfire and generate a sudden, uncontrolled surge of electrical activity in the brain more precisely in the cerebral cortex. There are three to six layers of neurons found to be present in the cerebral cortex with only three distinct layers; present in the hippocampus, which is located in the medial temporal lobe. The majority of the cortex has 6 distinct cell layers and covers most of the surface of the cerebral hemispheres, the hippocampus which consists of three major regions: subiculum, hippocampus proper (Ammon's horn) and dentate gyrus has three layers in the later two regions and a transitional three to six layers in the subiculum. Given the fact that the basic mechanism of neuronal excitability is the action potential, a hyperexcitable state can result from increased excitatory synaptic neurotransmission, decreased inhibitory neurotransmission, an alteration in voltage-gated ion channels, or an alteration of intra- or extra-cellular ion concentrations in favor of membrane depolarization. A hyperexcitable state can also result when several synchronous subthreshold excitatory stimuli occur, allowing their temporal summation in the post synaptic neurons (Avoli M et.al, 2001).

Action potentials occur due to depolarization of the neuronal membrane, with membrane depolarization propagating down the axon to induce neurotransmitter release at the axon terminal. The action potential occurs in an all-or-none fashion as a result of local changes in membrane potential brought about by net positive inward ion fluxes.

As mentioned earlier hippocampus is involved in epilepsy and a special group of cells known as granule cells (GCs) are the focus of the epilepsy research. These cells are present in dentate gyrus region of hippocampus and are primary gateway of cortical inputs to CA3 region of hippocampus (Crawford I L 1973). GCs act as filters and protect temporal lobe seizures by upregulation of inward rectifier $\mathrm{K}^{+}$channels and increase leak conductance in the granule cells of dentate gyrus (Young C et.al, 2009 and Stegan M et al 2009). Repetitive firing of action potential of inter neurons exhibits diverse repetitive firing behaviour depending upon their harboring levels in the granular layer of dentate gyrus (David M.D., et al., 1997). In epileptic patients, most of granule cells display hyperexcitable, non frequency adapted cells (Dietrich D et al. 1990) which are highly susceptible for the generation of temporal lobe epilepsy. Non adapted bursting cells are characterized by their ability to generate action potential without reducing firing frequency and less afterhyperpolarization (AHP). This type of burst firing have also been recorded in inferior colliculus neurons (Tan M L et al, 2007), CA3 pyramidal neurons (Peter H et al 2008) and in spinal cord neurons (Smith M and Perrier J, 2006). The non-adapted bursting firing may depend upon calcium influx (Anthony A G and Benjamin 1984) or due to the presence of persistent sodium currents (Yunru L et al, 2004). In regular frequency adapted neurons, intracellular $\mathrm{Ca}^{+2}$ increases (Knopfel T and Gahwiler B H, 1992) which activates the calcium gated potassium channels (Kca). These channels gradually decrease the positivity inside the cell and reduce the firing frequency of action potential in the later stages of Sustained Repetitive firing SRF. Buster cells (type of GCs) have low Kca channel density (Podloger M and Dirk D,2006) due to which they exhibits the continuously same or shorter spike 
duration throughout the pulse. Sodium and potassium channels are the main contributors of action potential and underlying cause of repetitive firing pattern (Joshua B C, et al 2000). In buster GCs of dentate gyrus, amplitude and firing frequency of action potential increases in response to number of pulses. $\mathrm{Na}^{+}$and $\mathrm{K}^{+}$voltage-gated channels change their voltage dependency for activation and inactivation in response to sustained repetitive firing (SRF). Inactivation of sodium channels is significantly modulated by growth factor (Chuan-ju L et al, 2003) and chronic compression (Zhi-Jiang H and Xu-Jun S, 2008). Similarly the potassium channel activation and inactivation are modulated by cAMP (Jian M et al, 1999), potassium channel-interacting proteins KChIPs (Frank A W et al, 2000) growth factor and tyrosine kinases (Mark B, 1997). Literature indicated that sustained repetitive firing can be good electrical tool to find out about the underlying cause of epilepsy. The present study was therefore undertaken to establish the role of underlying currents in SRF as to establish the importance of invitro models in understanding the mechanism of epilepsy. This is our recent unpublished data, exhibiting the interesting findings of the effect of repetitive stimulation on neuronal excitability and on the kinetics of voltage dependent sodium and potassium channels. We used postnatally cultured granule cells from dentate gyrus region of hippocampus from 1-5 day old rat pups to study the role of SRF in regulating voltage gated $\mathrm{Na}^{+}$and $\mathrm{K}^{+}$currents. These voltage gated monovalent cationic currents were recorded by patch clamping "control" and SRF-induced "epileptic cells" in whole cell configuration. We then measured the changes in the activation and inactivation of both channels before and after SRF in granule cells of dentate gyrus and observe a novel role of "window currents". Overlapping of channel activation and inactivation is referred as window current; a sustained current which is observed if a fraction of channel opens (Popen>0) when the probability for their inactivation is not zero. Here we report that the repetitive depolarizing stimuli alters the kinetics and gating of $\mathrm{Na}+$ and $\mathrm{K}+$ channels and bring the granule cell membrane to more hyper polarized potential. These electrical changes in the membrane of cultured granule cells may partially responsible for the better survival of granule cells in excitotoxic conditions. This data may put some light on the underlying homeostatic mechanism in epilepsy mainly in temporal lobe epilepsy.

\section{Material and methods}

\subsection{Chemicals}

All chemicals were purchased from Sigma (USA) and salts from Applichem (Denmark), except D-AP5, TTX, and CNQX disodium salt from Ascent Scientific (Weston-Super-Mare, United Kingdom) and $\mathrm{KCl}$ from Merck (Darmstadt, Germany).

\subsection{Hippocampal neuronal cell culture}

Animals were sacrificed for culturing post natal neurons in accordance with the guide lines of the animal ethic committee at the Panjwani Center for Molecular Medicine and Drug Research (PCMD), University of Karachi, Pakistan after getting animal ethic approval. Hippocampal dentate gyrus cells were cultured as described previously (Fatima Shad and Barry PH, 1998). Briefly, dentate gyri from both hemispheres were isolated under dissecting microscope from 1-5 days old specific pathogen free (SPF) Wister rats and plated at a density of $1 \times 10^{5}$ cells $/ \mathrm{cm}^{2}$ onto three $22 \mathrm{~mm}$ rounded cover slips previously coated with poly-L-lysine $(0.05 \mathrm{mg} / \mathrm{ml})$, and were placed in a $100 \mathrm{~mm}$ cell culture dishes. Cultures were 
maintained at $37^{\circ} \mathrm{C}$ in a humidified $5 \% \mathrm{CO}_{2}$ incubator and were fed twice a week with DMEM (Dulbecco's Modified Eagle's Medium) plus containing 85ml. DMEM, 1ml. L glutamine, $1 \mathrm{ml}$. Fungizone, $2 \mathrm{ml}$. Streptomycin/Penicillin, $1 \mathrm{ml}$. sodium pyruvate and $10 \mathrm{ml}$. Fetal Bovine Serum. The neuronal cultures were used from the fourth day for the patch clamp experiments.

\subsection{Patch clamp recordings}

One of the author (FSK) is using patch clamp technique for last more than twenty years, and the present method was a modified version of one of her earlier papers (Fatima Shad $\mathrm{K}$ and Barry PH, 1992). Briefly, one polylysinated $22 \mathrm{~mm}$ round cover slip with cultured cells grown on it was fitted in the bath chamber $(1 \mathrm{ml}$. capacity) and was mounted on the stage of an inverted microscope (Nikon TE2000-U, Tokyo, Japan). Cells were perfused continuously with Artificial Cerebrospinal Fluid (ACSF) containing (in $\mathrm{mM}$ ): $126 \mathrm{NaCl}, 3.5 \mathrm{KCl}, 25$ $\mathrm{NaHCO}_{3}, 1.2, \mathrm{NaH}_{2} \mathrm{PO}_{4}, 2 \mathrm{CaCl}_{2}, 1.3 \mathrm{MgCl}_{2}, 11$ glucose, and $\mathrm{pH}(7.3)$, and osmolarity was adjusted to $325 \pm 5 \mathrm{mOsm}$ with sucrose. Patch pipettes were pulled from borosilicate glass capillaries using a Brown-Flaming P-97 electrode puller (Sutter Instruments, USA) with a diameter 2-3 $\mu \mathrm{m}$. After fire polishing using micro forge, pippetes were filled with a solution containing (in mM): $145 \mathrm{KCl}, 10 \mathrm{NaCl}, 10$ EGTA, $1 \mathrm{MgCl}_{2}, 2 \mathrm{CaCl}_{2}$ and $10 \mathrm{HEPES},(\mathrm{pH}$ 7.2) and had a tip resistance of of 4 to $5 \mathrm{M} \Omega$. Osmolality was adjusted to $300 \pm 10 \mathrm{mOsm}$ with sucrose. Patch clamp recordings were carried out using HEKA EPC 10 dual pre amplifiers (HEKA Instruments, Inc., New York.) placed on coarse adjustment plates of automated micromanipulators (Scientifica) at either side of the microscope. Data was recorded and analysed using PatchMaster, and temperature was maintained at $25 \pm 0.5$ by TC-20 temperature controller (ALA Scientific Instruments). Data was filtered with values of 8 and10 kHz and digitized at 10 and $20 \mathrm{kHz}$ for current and voltage clamp respectively. Membrane capacitance $(\mathrm{Cm})$ was recorded by using built in LockIn software after calculating the cell size and getting the current density. The offset potential was canceled before patching the cell and was re-checked after each recording for any drift. Leakage currents, determined by application of small hyperpolarizing commands were substracted automatically from the response.

Most pharmacological experiments for the isolation of sodium and potassium currents were conducted in the presence of tetrodotoxin (TTX) and synaptic blockers, that is, D (-)-2amino-5-phosphonopentanoic acid (D-AP5), 1, 2, 3, 4-tetrahydro-7-nitro-2,3dioxoquinoxaline-6-carbonitrile disodium (CNQX), and picrotoxin (PTX). In $\mathrm{K}^{+-}$ replacement experiments, cells were first recorded with normal intracellular solution and then with $\mathrm{K}^{+}$-free intracellular solution. In these cases $\mathrm{K}+$ was replaced with equimolar tetraethyl ammonium (TEA) in the ACSF and the pipette solution contained (in $\mathrm{mM}$ ): 135 TEACl, $20 \mathrm{CsCl}, 0.1$ EGTA, $2 \mathrm{MgCl}^{2}$, and $2 \mathrm{Na}^{2} \mathrm{ATP}$ (pH 7.28 adjusted with TEA hydroxide). Chemicals such as CNQX, D-AP5, TEACl, and TTX were kept in $\mathrm{H}_{2} \mathrm{O}$ stocks at $20^{\circ} \mathrm{C}$ and PTX was kept in dimethylsulfoxide (DMSO) stocks at $20^{\circ} \mathrm{C}$. Final concentrations were diluted freshly in oxygenated recording ACSF (DMSO 1:1,000) and subsequently kept in syringes of perfusion system (Octaflow, ALA Scientific, U.S.A.) under nitrogen pressurized at 1.3-1.6 bar before bath application.

Whole cell recording of GCs were obtained both in current and voltage clamped mode after checking their resting membrane potential (RMP). Only those cells having RMP more negative than $-50 \mathrm{mV}$ were used. Both inward and outward currents were observed in response to +60 to $-60 \mathrm{mV}$ pulses of $10 \mathrm{mV}$ steps. 
Following diagrams display some raw data to create more understanding about the process of patch clamping and how the windows of the HEKA EPC10 amplifier looks like and what are the parameters constantly regulated by the amplifier itself. Please note the gain values as well as C-fast and C-slow values (fast and slow capacitative transients) automatically adjusted by the amplifier along with liquid junction potential and series resistance.

Symmetrical Sodium solution in response to voltage steps from $-60 \mathrm{mV}$ to $+60 \mathrm{mV}$

Cell 1

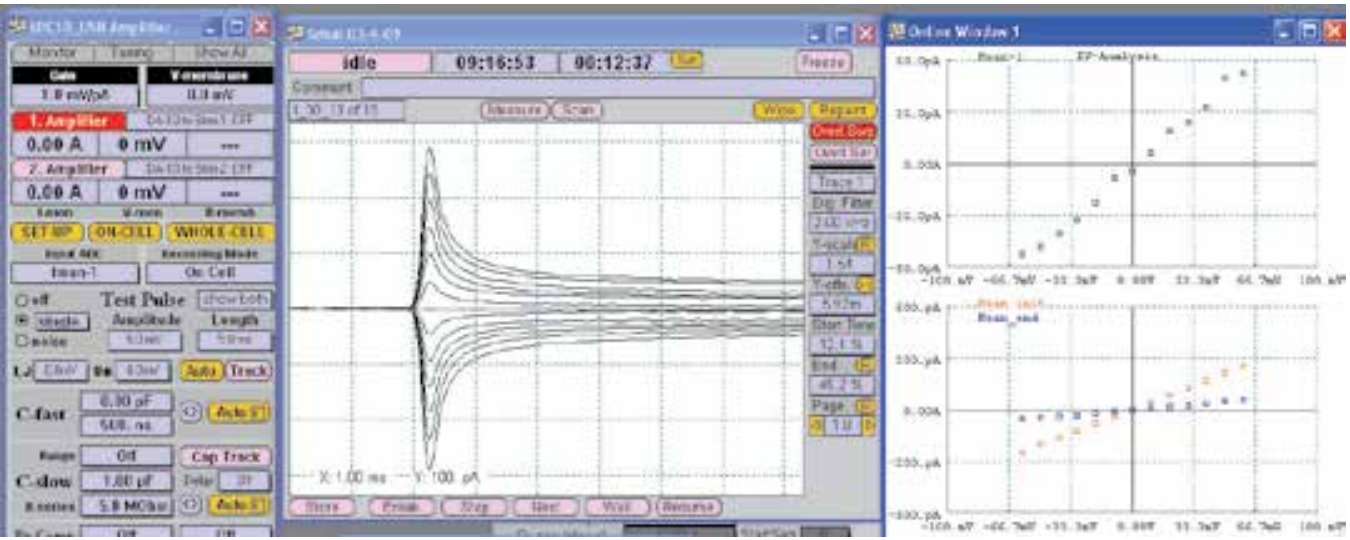

Cell 2

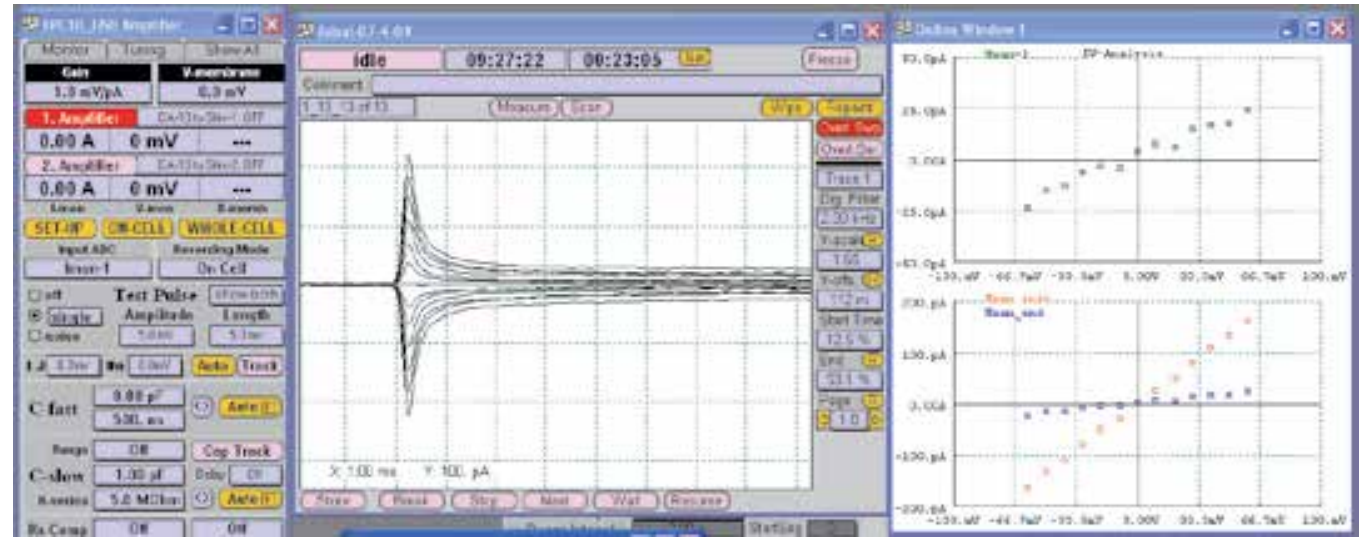




\section{Low sodium}

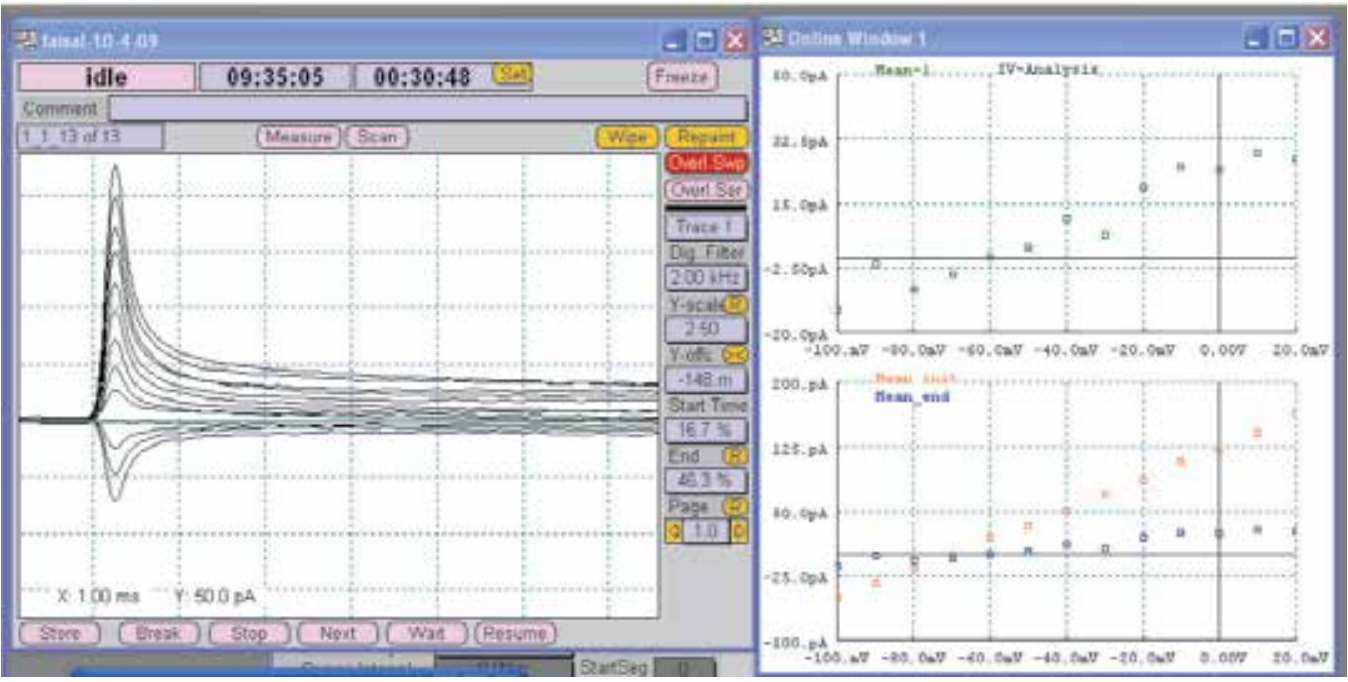

Another patch clamped cell
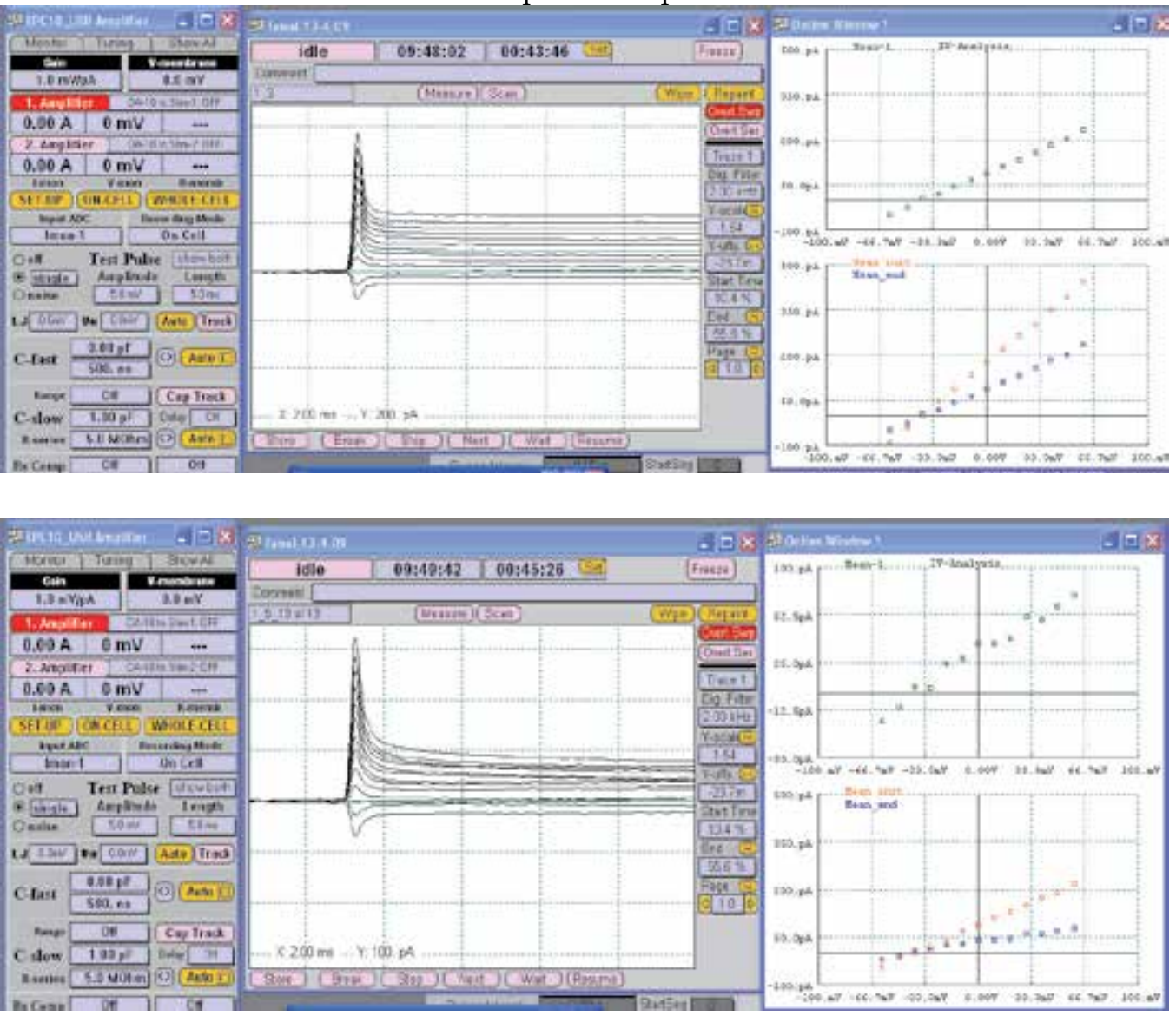


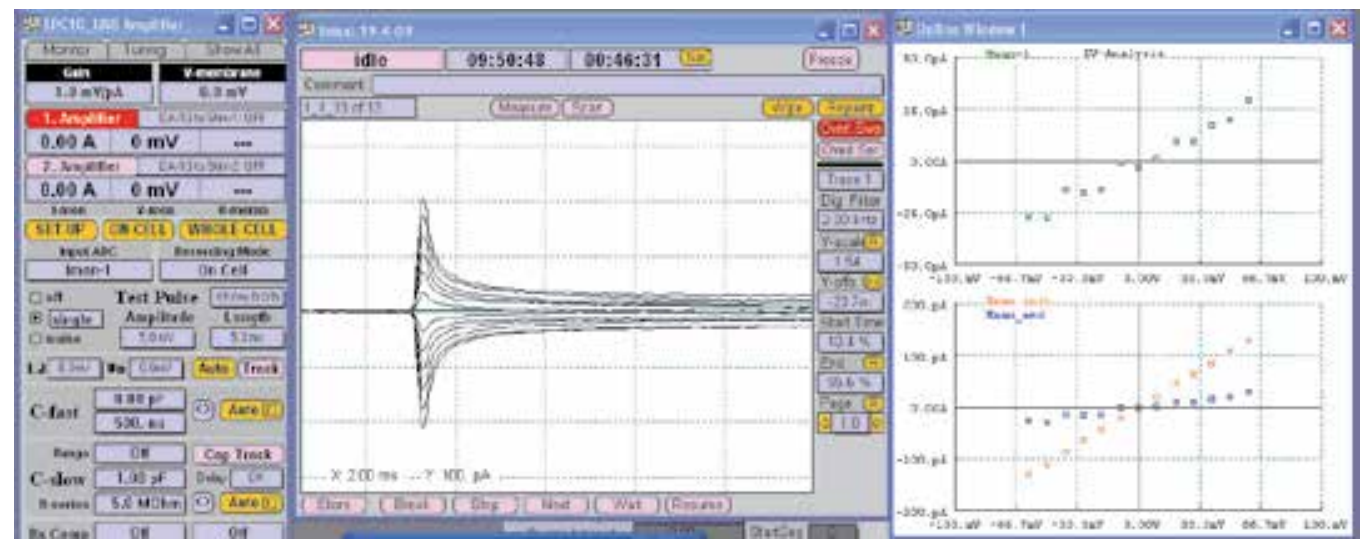

Absence of inward currents
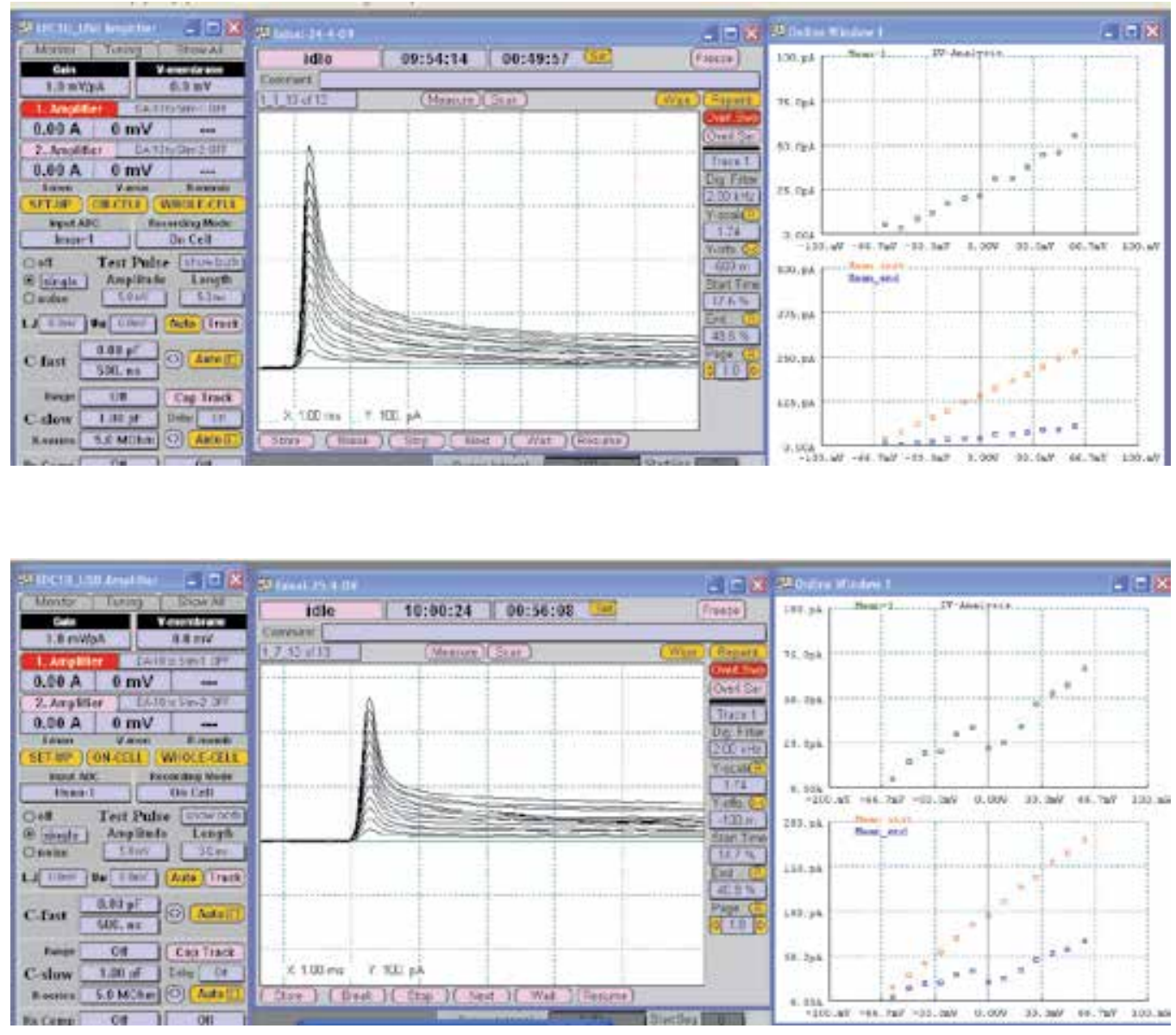

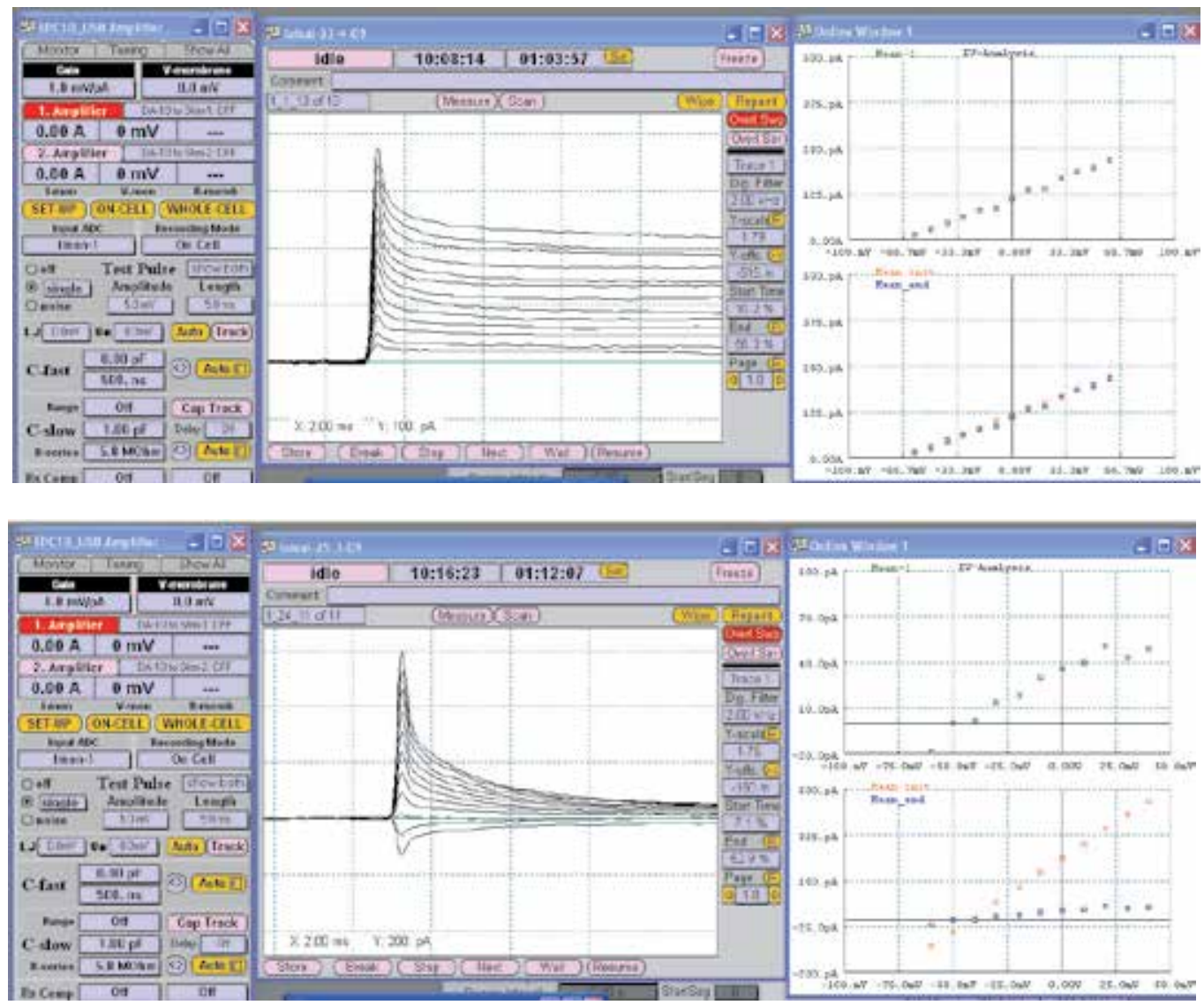

Fig. 1. In the above figures (A-J) presenting raw data (directly from the computer) simply exhibiting that how different cells behave differently and how changes in ionic concentration changes the inward and outward deflection of currents. Literature indicated a variety of approaches for in vitro modeling of epilepsy mainly temporal lobe epilepsy. One can design an epileptic model by using chemical (absence of $\mathrm{Mg}$ ) or electrical (SRF) tool. These artificial epileptic models are capable of mimicking the silent features of this pathology and can be tested for novel therapeutic agents but with great caution during their interpretation.

We thought it will be beneficial for non expert readers to see how a cell can be patched and how the values of transient and steady state currents look like in response to voltage pulses. One can use the following values to draw a current voltage graph as well as the following equation can be used for practice to see which ion is more permeable for the non selective cation or anion channel. Erev $=$ is the voltage at which the concentration of that particular ion is equal in ECF and ICF and after this voltage the ion will change its direction, $\mathrm{z}$ is the valency and $[\mathrm{A}]_{\mathrm{o}}$ are the $[\mathrm{A}] \mathrm{i}$ are the concentration out side and inside. $\mathrm{PA}$ and $\mathrm{PB}$ are the permeability of ion A and ion B

Sodium currents (Number of Sodium channels, permeability of $\mathrm{Na}$ ions per unit change in voltage and reversal potential of the sodium or any other ion can be calculated using the following equation)

$$
\text { Erev }=\mathrm{RT} / \mathrm{zF} \text { In PA }[\mathrm{A}]_{\mathrm{o}} / \mathrm{PB}[\mathrm{A}] \mathrm{i}
$$



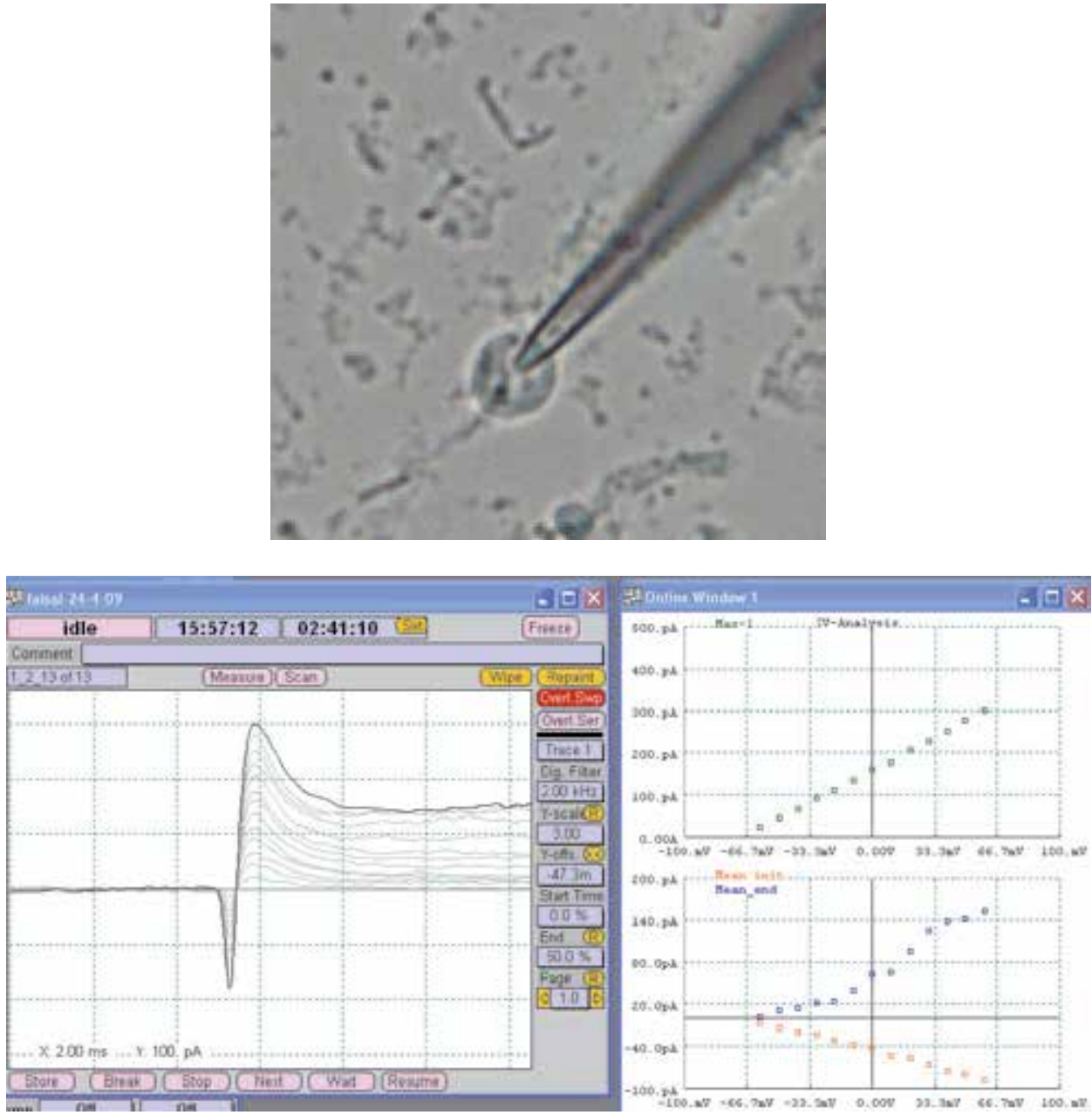

\begin{tabular}{|c|c|c|c|c|c|}
\hline$\#$ & $\begin{array}{c}\text { Holding Potential } \\
(\mathrm{mV})\end{array}$ & $\begin{array}{c}\text { peak (o) } \\
\mathrm{I}(\mathrm{pA})\end{array}$ & $\begin{array}{c}\text { inward } \\
\mathrm{I}(\mathrm{pA})\end{array}$ & $\begin{array}{c}\text { Persist (o) } \\
\mathrm{I}(\mathrm{pA})\end{array}$ & $\begin{array}{c}\text { Time to peak (i) } \\
\mu \text { sec. }\end{array}$ \\
\hline 1 & -60 & 22.567 & -6.9942 & 2.0444 & 265.21 \\
\hline 2 & -50 & 45.241 & -13.975 & 11.293 & 276.52 \\
\hline 3 & -40 & 65.281 & -20.338 & 13.925 & 275.34 \\
\hline 4 & -30 & 91.400 & -23.784 & 22.002 & 273.24 \\
\hline 5 & -20 & 110.64 & -31.609 & 23.278 & 272.97 \\
\hline 6 & -10 & 135.58 & -38.035 & 39.726 & 275.55 \\
\hline 7 & 0 & 161.08 & -42.521 & 61.990 & 274.19 \\
\hline 8 & 10 & 176.76 & -53.981 & 65.602 & 275.32 \\
\hline 9 & 20 & 205.64 & -57.132 & 95.058 & 278.12 \\
\hline 10 & 30 & 227.72 & -66.613 & 125.31 & 275.80 \\
\hline 11 & 40 & 250.62 & -76.349 & 137.64 & 277.57 \\
\hline 12 & 50 & 278.18 & -81.710 & 140.65 & 277.32 \\
\hline 13 & 60 & 300.42 & -88.700 & 152.24 & 276.56 \\
\hline
\end{tabular}




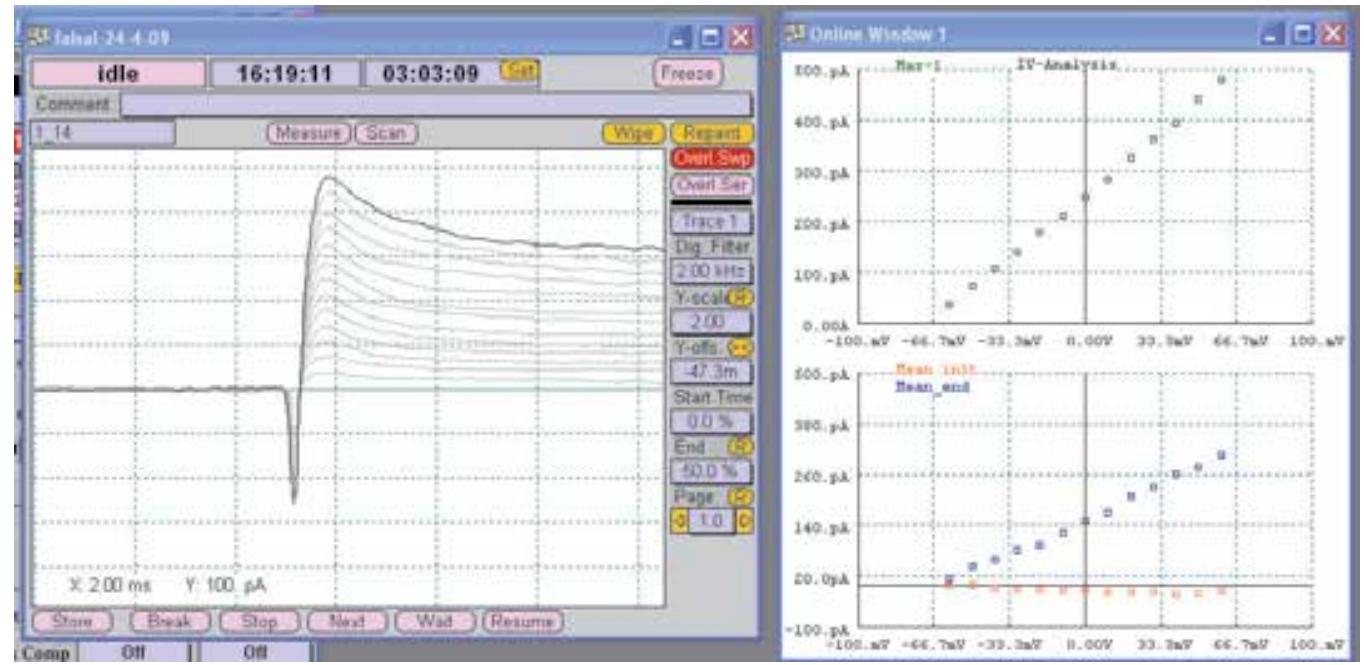

\begin{tabular}{|c|c|c|c|c|c|}
\hline$\#$ & $\begin{array}{c}\text { Holding Potential } \\
(\mathrm{mV})\end{array}$ & $\begin{array}{c}\text { peak (o) } \\
\mathrm{I}(\mathrm{pA})\end{array}$ & $\begin{array}{c}\text { Inward } \\
\mathrm{I}(\mathrm{pA})\end{array}$ & $\begin{array}{c}\text { Persist (o) } \\
\mathrm{I}(\mathrm{pA})\end{array}$ & $\begin{array}{c}\text { Time to peak } \\
\text { (i) sec. }\end{array}$ \\
\hline 1 & -60 & 37.013 & -1.3052 & 15.280 & 500.00 \\
\hline 2 & -50 & 72.857 & -1.6571 & 41.372 & 500.00 \\
\hline 3 & -40 & 107.55 & -7.0724 & 59.973 & 500.00 \\
\hline 4 & -30 & 140.62 & -11.647 & 80.917 & 500.00 \\
\hline 5 & -20 & 179.49 & -9.6198 & 93.364 & 500.00 \\
\hline 6 & -10 & 211.62 & -14.327 & 122.26 & 500.00 \\
\hline 7 & 0 & 249.49 & -12.186 & 149.24 & 500.00 \\
\hline 8 & 10 & 282.44 & -15.985 & 170.72 & 500.00 \\
\hline 9 & 20 & 324.09 & -17.815 & 207.99 & 500.00 \\
\hline 10 & 30 & 363.06 & -16.406 & 230.13 & 500.00 \\
\hline 11 & 40 & 394.45 & -23.742 & 261.85 & 500.00 \\
\hline 12 & 50 & 442.30 & -20.464 & 277.07 & 500.00 \\
\hline 13 & 60 & 478.56 & -13.076 & 306.75 & 500.00 \\
\hline
\end{tabular}

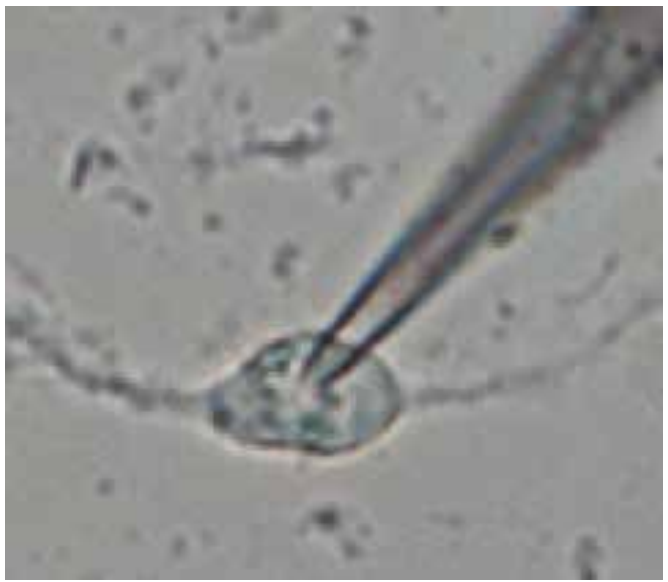




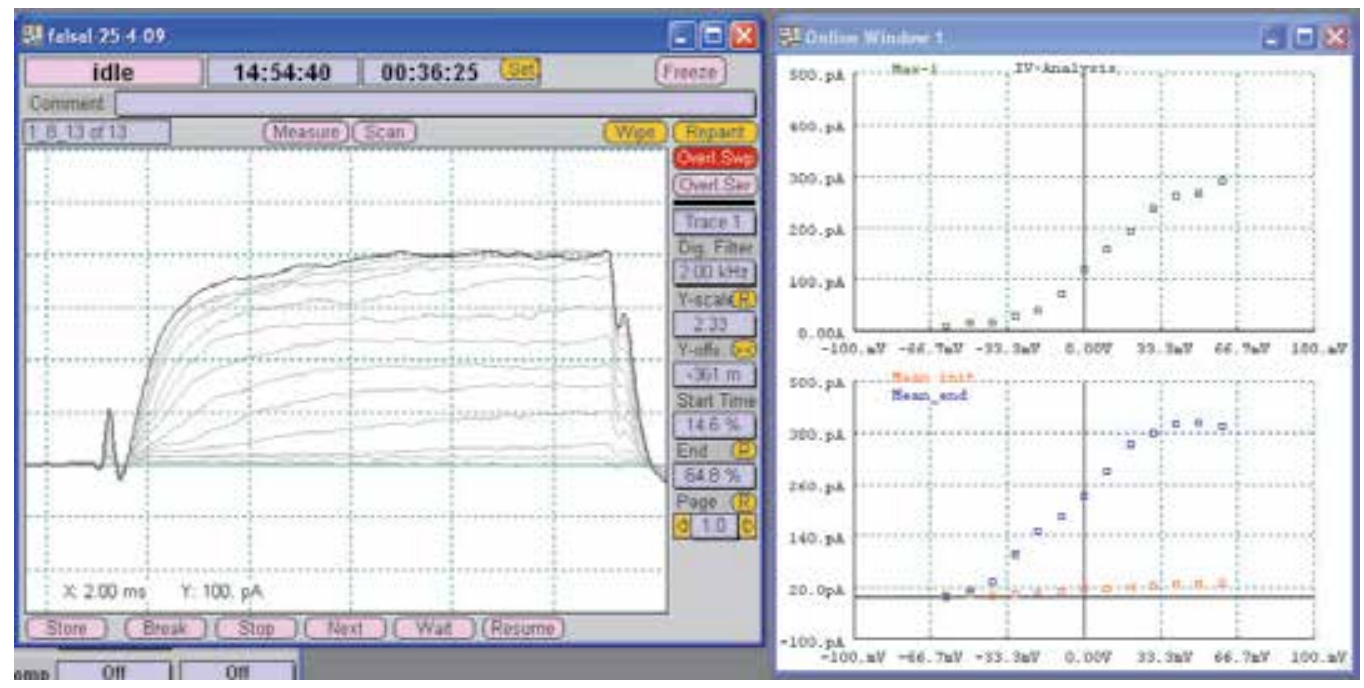

\begin{tabular}{|c|c|c|c|c|c|}
\hline$\#$ & $\begin{array}{c}\text { Holding Potential } \\
(\mathrm{mV})\end{array}$ & $\begin{array}{c}\text { peak (o) } \\
\mathrm{I}(\mathrm{pA})\end{array}$ & $\begin{array}{c}\text { inward } \\
\mathrm{I}(\mathrm{pA})\end{array}$ & $\begin{array}{c}\text { Persist (o) } \\
\mathrm{I}(\mathrm{pA})\end{array}$ & $\begin{array}{c}\text { Time to peak } \\
\text { (i) sec. }\end{array}$ \\
\hline 1 & -60 & 7.5033 & 1.7111 & -84.261 & 249.24 \\
\hline 2 & -50 & 15.568 & 5.0703 & 13.233 & 258.98 \\
\hline 3 & -40 & 16.076 & 1.9596 & 34.109 & 253.96 \\
\hline 4 & -30 & 27.778 & 5.7529 & 96.613 & 252.26 \\
\hline 5 & -20 & 39.568 & 9.0225 & 149.87 & 253.74 \\
\hline 6 & -10 & 71.728 & 9.6337 & 184.22 & 250.65 \\
\hline 7 & 0 & 119.43 & 16.273 & 231.95 & 249.77 \\
\hline 8 & 10 & 158.85 & 18.046 & 290.64 & 248.82 \\
\hline 9 & 20 & 193.04 & 21.480 & 353.00 & 248.69 \\
\hline 10 & 30 & 239.25 & 25.096 & 379.17 & 252.02 \\
\hline 11 & 40 & 261.33 & 27.338 & 403.03 & 251.91 \\
\hline 12 & 50 & 267.82 & 26.792 & 404.47 & 252.70 \\
\hline 13 & 60 & 290.25 & 31.005 & 395.27 & 249.12 \\
\hline
\end{tabular}

Fig. 2. In the above table and figures bipolar cells were patch clamped and currents were measured in response to the voltage pulses. Despite of the fact that both cells used were similar and pulse protocol applied was exactly the same but the responses were quite different for both transient and sustained currents, indicating the existence of heterogeneity of the current population in each cell. This again warns us for the presence of significant differences in the same type of cells experiencing the similar electrical stimulus.

Basically these diagrams and tables are given for those who are interested in practicing for making current voltage (I/V) curves for the understanding of the phenomenon of normal impulse as well as the mechanism of epileptic train.

We hope by now its clear that sodium and potassium are the major cations involved in the process of action potential and a high frequency series of action potentials are knows as epileptic episodes. Both $\mathrm{Na}$ and $\mathrm{K}$ gated currents go through the process of activation and inactivation. These currents when recorded before and after the application of SRF gives two 
very different kinetic profiles and this information will be helpful in detecting the cell membrane status its protein channel and their integirity. To analyze the voltage dependence of channel activation, the conductance $(\mathrm{G})$ was calculated by using the equation:

$$
\mathrm{G}=\mathrm{I} /(\mathrm{Vm}-\mathrm{Vrev})
$$

Where I is the peak current, $\mathrm{Vm}$ is the test pulse voltage and Vrev is the reversal potential of under study ion. Conductance was plotted against Vm and fit to a Boltzmann distribution equation:

$$
\mathrm{G}=\mathrm{Gmax} /\left(1+\exp \left(\mathrm{V}_{1 / 2^{-}} \mathrm{Vm}\right) / \mathrm{k}\right)
$$

Where Gmax is the maximum conductance, $\mathrm{V}_{1 / 2}$ is the potential at which activation is half maximal, and $\mathrm{k}$ is the slope of the curve.

For the estimation of channel inactivation, the inactivation parameters were fitted to Boltzmann distribution equation:

$$
\mathrm{I} / \operatorname{Imax}=1 /\left(1+\exp \left(\mathrm{V}_{1 / 2}-\text { Vpre }\right) / \mathrm{k}\right)
$$

Where Imax is the maximum current after the most hyperpolarized prepulse, the Vpre is the prepulse potential, $\mathrm{V}_{1 / 2}$ is the potential at which inactivation is half-maximal.

\subsection{Statistics}

Significance of differences were tested by Student's $t$-test with $P<0.05$ considered to be significant.

\section{Results}

Dentate gyrus granule cells of hippocampus were plated at a concentration of $1 \times 10^{5}$ cells $/ \mathrm{cm}^{2}$ per plate (containing three $22 \mathrm{~mm}$ round cover slips). These cells were identified mostly to be granule cells ( ${ }^{*} \mathrm{Fig} .3 \mathrm{~B}$ right panel). Only shiny healthy intact cells were used for patch clamp experiments. All granule cells of dentate gyrus have similar passive membrane properties (Table. 1) and are of very homogenized morphological properties.

( ${ }^{*}$ Though Fig 3 was suppose to be Fig 1 of the result section)

\begin{tabular}{|l|c|c|}
\hline & Pre SRF & Post SRF \\
\hline Input Resistance $(\mathrm{M} \Omega)$ & $263.1 \pm 25.2$ & $192.6 \pm 30.6$ \\
\hline Resting Membrane Potential, RMP $(\mathrm{mV})$ & $-63 \pm 5.3$ & $-62 \pm 4.7$ \\
\hline Threshold for action potential $(\mathrm{mV})$ & $-49 \pm 2.6$ & $-42 \pm 6.6$ \\
\hline Spike Amplitude $(\mathrm{mV})$ & $90 \pm 10.4$ & $72 \pm 12.2$ \\
\hline No of Spikes in the burst & $15 \pm 2$ & $11 \pm 3$ \\
\hline After hyperpolarization, Amplitude $(\mathrm{mV})$ & $1.1 \pm 0.2$ & $1.8 \pm 0.3$ \\
\hline
\end{tabular}

Table 1. Membrane properties of granule cells of mammalian dentate gyrus 
After patch clamping the cells for the measurment of their passive membrane properties and their morphological features. We have decided to use them for our in vitro model of epilepsy. For that purpose we have used an electrical tool called Sustained repetitive firing (SRF), a protocol of depolarizing pulses of $0.5 \mathrm{nA}$ amplitude with $500 \mathrm{~ms}$ duration and of 0.3 $\mathrm{Hz}$ frequency as to mimic epileptic burst in the cultured granule cells. We have used HEKA amplifier pulse protocol program to generate SRF and introduced it into the clamped cell through the recording electrode. In response to SRF pulse, patched cell exhibit regular and continues series of action potentials mimicking an epileptic episode as been observed earlier (DeLorenzo RJ et al, 2000). The duration and frequency of SRF pulses were remains constant for all experiments. Those cells which were patched in whole cell configuration (with out any SRF) were considerd as "control cells". The patch clamped cultured neuronal cells were also tested to confirm that the SRF induced hyperexcitability effect is activity and not time dependent. For testing this phenomenon, the cell was patch clamped in whole cell configuration without any stimulus for a duration of approximately eight seconds (equivqlent to the time of three depolarized pulses) then the SRF pulses were injected (Fig. 3).

A

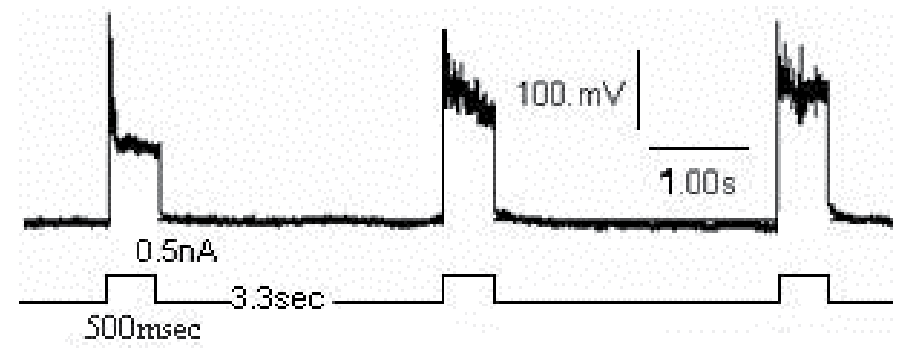

B
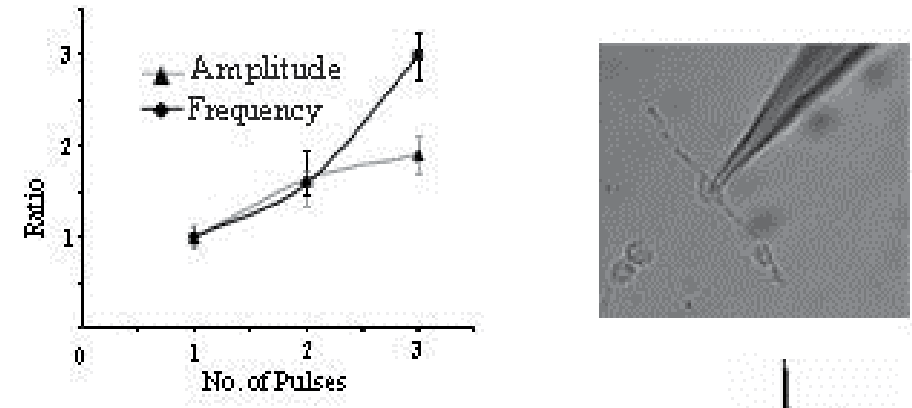

$\mathrm{C}$

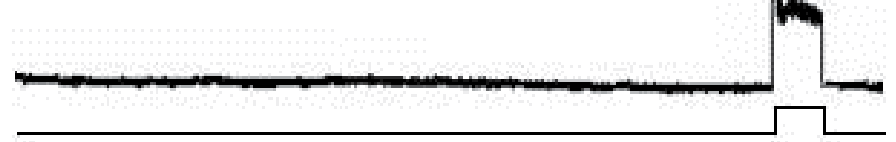

Fig. 3. Effect of SRF on frequency and amplitudes of action potential in granule cells Spike amplitude and firing frequency of action potentials were increased as a result of SRF depolarizing pulses of $0.3 \mathrm{~Hz}$. A: In response to sustained repetitive pulses of $0.5 \mathrm{nA}$, a train of action potential was observed in a patch clamped granule cell in the current clamp mode of whole cell configuration. B: exhibits the relationship between the number of pulses with spike amplitude and frequency, inset showing a patch clamped granule cell. C: demonstrates the voltage response of SRF after a delay of $8 \mathrm{sec}$ confirming that the increase in amplitude and frequency of action potential is activity not time dependent. 
After calculating the activation and inactivation kinetics by using the formulas mentioned earlier, we have found that the value of half maximum activation $\mathrm{V}_{1 / 2}$ for $\mathrm{Na}+$ was $-25.8 \pm 3.2$ $\mathrm{mV}$ before and increased to- $29 \pm 4.1 \mathrm{mV}$ after SRF. Whereas for Ksus (sustained K currents) values were $-30 \pm 4.3 \mathrm{mV}$ before and was changed to $-33 \pm 5.1 \mathrm{mV}$ after SRF (Fig. $4 \mathrm{~A}$ ) but transient potassium activation $V_{1 / 2}=-30.4 \pm 2.8 \mathrm{mV}$ significantly get changed to $-41 \pm 5.2 \mathrm{mV}$ after SRF. The transient potassium current $\left(\mathrm{K}_{\mathrm{t}}\right)$ was not decreased in the presence of $10 \mathrm{mM}$ tetraethylammonium (TEA) in the extra cellular solution but $4 \mathrm{AP}$ in the bath reduces this current up to $75 \%$.

\section{Voltage dependent of sodium current before and after SRF}

\section{A: Activation}

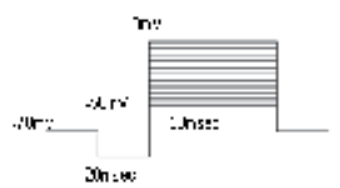

B: Inactiration

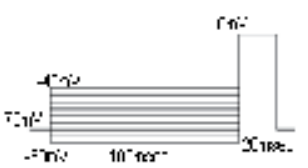

C: window current

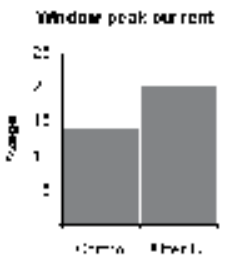

Hh:lun: Sitt
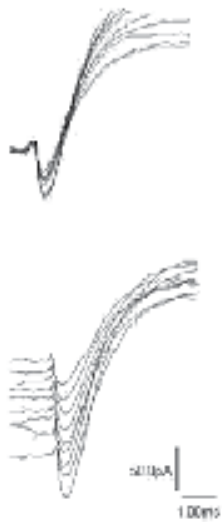

A.thy S.:P
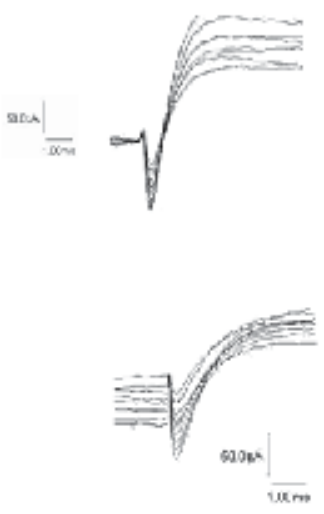

Voltag 8 dep endance of sodum Gument betore and atter Skrt

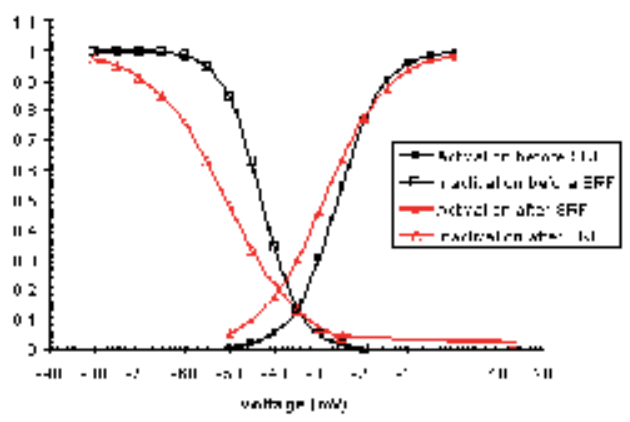

Fig. 4. A: Left hand panel exhibits activation pulse protocol (from $-50 \mathrm{mv}$ to $0 \mathrm{mV}$ ) and right hand graph shows Na+ activation current response before and after SRF B: Steady state inactivation pulse protocol on the left before and after SRF current recordings on the right. C: Left panel shows voltage dependency of $\mathrm{Na}+$ activation and inactivation currents. Right panel shows histogram of changes in window currents before and after SRF, drawn from the activation-inactivation curves. Peak window current was observed at $-35 \mathrm{mV}$ which was shifted to $-39 \mathrm{mV}$ after SRF. $\mathrm{V}_{1 / 2}$ for activation before SRF was calculated to $-25.8 \mathrm{mV}$ and for inactivation $-42.7 \mathrm{mV}$ which were also shifted to $-29 \mathrm{mV}$ and $-50.6 \mathrm{mV}$ respectively after SRF. 
Similarly $\mathrm{V}_{1 / 2} \mathrm{mV}$ values of $\mathrm{Na}+$ inactivation $=-42.7 \pm 6.6 ; \mathrm{Kt}=-68.5 \pm 5.6$; and $\mathrm{Ksus}=-58 \pm 5.6$ are markedly shifted towards - ve potential, $\mathrm{Na}+=-50.6 \pm 7.4 ; \mathrm{Kt}=-75.6 \pm 8.2 ; \mathrm{Ksus}=-63 \pm 9.4$. These changes in voltage dependency of activation and inactivation currents results in an increase in sodium and decrease in potassium window currents. (Table 2)

\begin{tabular}{|c|c|c|c|c|c|c|c|}
\hline & \multicolumn{2}{|c|}{ Activation } & \multicolumn{2}{|c|}{ Inactivation } & \multirow[b]{2}{*}{$\begin{array}{l}\text { Window } \\
\text { Peak-Volt }\end{array}$} & \multirow[b]{2}{*}{$\begin{array}{l}\text { Window } \\
\text { range }\end{array}$} & \multirow[b]{2}{*}{$\begin{array}{c}\text { Window } \\
\text { Peak }\end{array}$} \\
\hline & $\mathrm{V}_{1 / 2 \text { (act) }}$ & $\mathbf{K}_{\text {(activation) }}$ & $\mathrm{V}_{1 / 2 \text { (inactivation) }}$ & $\begin{array}{c}\mathbf{K}_{\text {(inactiva- }} \\
\text { tion) }\end{array}$ & & & \\
\hline & $(m V)$ & & $(m V)$ & & $(m V)$ & $(m V)$ & $\%$ \\
\hline \multicolumn{8}{|c|}{ Sodium Current } \\
\hline 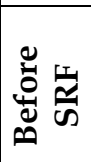 & $-25.8 \pm 3.2(12)$ & $5 \pm 0.5$ & $-42.7 \pm 6.6(8)$ & $4.2 \pm 1.1$ & -35 & -45 to -25 & 14 \\
\hline 起峦 & $-29 \pm 4.1(11)$ & $7.2 \pm 0.6$ & $-50.6 \pm 7.4(6)$ & $8.3 \pm 0.9$ & -39 & -54 to -20 & 20 \\
\hline \multicolumn{8}{|c|}{ Potassium Transient } \\
\hline 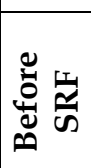 & $-30.4 \pm 2.8(10)$ & $6.6 \pm 0.8$ & $-68.5 \pm 5.6(9)$ & $7 \pm 1.2$ & -51 & -60 to -40 & 10 \\
\hline 离紊 & $-41 \pm 5.2(10)$ & $5 \pm 1$ & $-75.6 \pm 8.2(9)$ & $8 \pm 1.3$ & -55 & -60 to -45 & 8 \\
\hline \multicolumn{8}{|c|}{ Potassium Sustained } \\
\hline 怤步 & $-30 \pm 4.3(10)$ & $8.9 \pm 1.1$ & $-58 \pm 5.6(9)$ & $11 \pm 1.5$ & -35 & -60 to -10 & 20 \\
\hline 这紊 & $-33 \pm 5.1(9)$ & $8 \pm 0.6$ & $-63 \pm 9.4(8)$ & $9.2 \pm 1.3$ & -39 & -60 to -20 & 15 \\
\hline
\end{tabular}

Table 2. Voltage dependence of activation and inactivation before and after SRF in mammalian granule cells. 
$\mathrm{V}_{1 / 2 \text { (act): }}$ is the value of the voltage at which membrane reaches $50 \%$ of the maximum activation state

$\mathrm{V}_{1 / 2}$ (inact): Is the value of the voltage at which membrane reaches $50 \%$ of the maximum inactivation state

$\mathrm{K}(\mathrm{act})$ : slope of the activation curve

$\mathrm{K}$ (inact): slope of the inactivation curve

Window peak volt: Voltage at which window current reaches its peak.

SRF: Sustained Repetitive Firing

The number in the parenthesis indicate the number of experiments

$\mathrm{P}<0.01$ indicate significant differences compared with control group

These cells show non adapting firing frequency in response to superathreshold $(0.5 \mathrm{nA})$ current pulses (Fig.5A).

\section{Voltage dependent of potassium current before and after SRF}

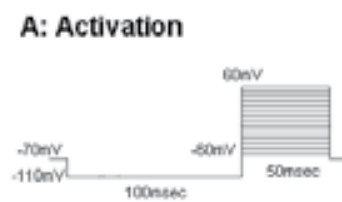

B: Inactivation

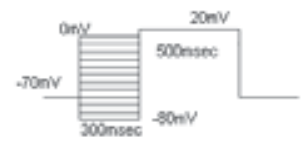

C: window current

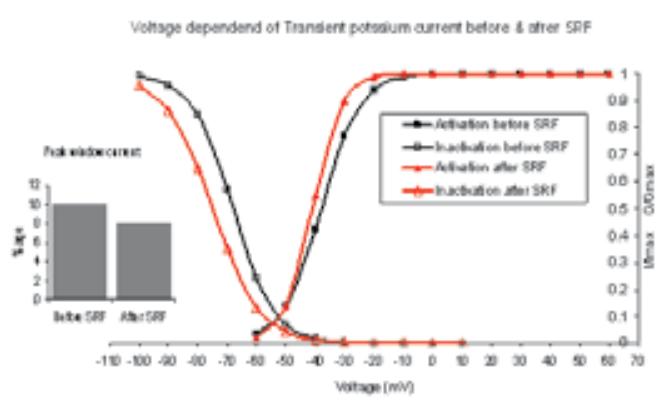

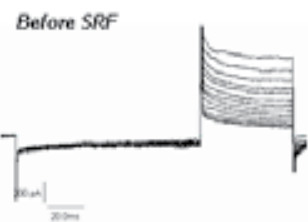

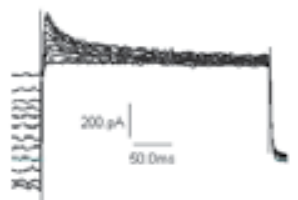

Aher SRF
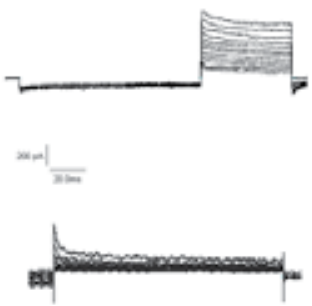

Fig. 5. This figure exhibits both sustained and transient potassium currents A: Activation pulse protocol is on the left, control current recording in the middle and after SRF in the right panel B: Exhibits (from left to right) inactivation pulse, control and after SRF current responses, for potassium channels. C: Voltage dependence of activation and inactivation of transient and sustained potassium currents. Histogram (in the inset) shows percentage of window currents before and after SRF. Amplitude of window current for transient potassium was at $-51 \mathrm{mV}$ which was shifted to $-55 \mathrm{mV}$ and sustained peak window current was shifted from $-35 \mathrm{mV}$ to $-39 \mathrm{mV}$ after SRF indicating voltage dependency of both type of potassium currents. 
For measuring the activity dependent increase in amplitude and firing frequency, we applied three consecutive current pulses at $0.3 \mathrm{~Hz}$ (inter pulse duration of $3.3 \mathrm{sec}$ ). We observed that the amplitude and frequency of spikes in the next pulse are greater then previous one (Fig. 5C). Amplitude and frequency increased with the ratio of 1.0:1.6:1.9 and 1.0:1.6:3.0 for three pulses respectively. Ratio of amplitude and frequency at the second pulse (after $4 \mathrm{sec}$ ) is almost equal but during third pulse (after $8 \mathrm{sec}$ ), the ratio for frequency after SRF get increase significantly (Fig. 5B). Amplification of amplitude and number of spikes were not found to be present when only one pulse was given for the same duration of the three pulses (approximately $8 \mathrm{sec}$ ) indicating that these changes in spikes were not time but activities dependent (Fig. 5D).

\section{Discussion}

The present study investigated the electrophysiological profile of granule cells and role of voltage gated $\mathrm{Na}+$ and $\mathrm{K}+$ channels in response to sustained repetitive firing. Amplitude and firing frequency of action potential for cultured granule cells were increased with increasing number of SRF pulses. We observed the voltage dependency of activation and inactivation of both $\mathrm{Na}+$ and $\mathrm{K}+$ currents after repetitive firing indicating both ionic currents have some underlying role in the modulation of action potential train in resposne to SRF. We have observed that both $\mathrm{Na}^{+}$and $\mathrm{K}^{+}$inactivation traces become less leaky after the exposure of SRF. We also observed that $\mathrm{Na}^{+}$channel inactivation and $\mathrm{K}^{+}$current activation has been shifted to more negative values indicting the reduce excitabilities in these cells after sustanined repetitive firing. Similar observation was also been made by (Christina C et al 2009 and Stegan M et al 2009) and ascribed that granule cells become leaky with Decreasing severity of temporal lobe epilepsy. Thus similar to our conclusion both authors suggested that seizures triggers expression of different neuroprotective reactions, including upregulation of $\mathrm{K}^{+}$channel density.

Literature also indicated that the levels of $\mathrm{Ca}^{+2}$ may gradually get increased intracellularly in response to SRF. Increased intracellular $\mathrm{Ca}^{+2}$ levels then may triggers the calcium gated potassium channels inducing after-hyperpolarization (AHP) in normal frequency adapting cells. Because of the low density of calcium gated potassium channels in granule cells of dentate gyrus, less adaptation of firing frequency may observed in some cases. Decrease levels of extracellular calcium due to repetitive firing results in sodium persistent current $\left(\mathrm{I}_{\mathrm{NaP}}\right)$ (Hailing $\mathrm{S}$ et al 2001). $\mathrm{I}_{\mathrm{NaP}}$ is capable of amplifying a neuron's response to synaptic input and enhancing its repetitive firing capability (Carl E S 2007). These granule cells are thought to play an important role in electrical signaling, in neuronal synchronization, and rapid information transmission (Adam $\mathrm{K}$ et al 2002) and their number found to be increased in the epileptic hippocampus. Similarly, increase intracellular calcium levels results into many changes in the cell such as increased cytosolic cAMP, which inturn significantly increases persistent sodium currents (Bibiane $\mathrm{F}$ et al, 1997) as well as inducing prolonged neuronal plasticity (Nikitin ES et al 2006). Literature indicated that sodium inactivation gates are involved in the repetitive firing response and the impairment of the inactivation results in an increase in whole-cell persistent current (Kristopher M.K. et al, 2006). PKA phosphorylate the inactivation gates of sodium channels which in turn prolong their activation state and enhances repetitive firing. $\beta 1$ subunit of the $\mathrm{Na}+$ channels seems to be involved in the modification of gating properties (Earl PD et al 1994). Similarly $\beta 4$ subunit of potassium channel observed to be participating in reducing dentate gyrus excitability and 
resultant protection against temporal lobe seizures (Robert $B$ et al 2005). When $\beta 4$ subunits of potassium channel get phosphorylated they alter the channel conductance and decrease the efflux of potassium and hence resultant increased positivity inside the cell. Our findings of shifting of window currents of granule cells to hyperpolarized voltages may mimic in vivo condition during epileptic seizures. Our results also reveals a complex dynamics behind repetitive spike discharge and suggests that a persistent $\mathrm{Na}^{+}$current plays an important role in action potential initiation and in the regulation of dentate gyrus granule cells transmission. The resting leak conductance was found to be doubled in epileptic granule cells and roughly $70-80 \%$ of this difference was sensitive to $\mathrm{K}+$ replacement. SRF exposed "epileptic granule cells" had strongly enlarged inwardly rectifying currents with a low micro molar $\mathrm{Ba}^{2+}$ sensitivity. Further investigations needed to be done in this regard. Beside SRF in conjunction with cultured granule cells, we have other tools in hand as to explore underlying mechanism of epilepsy and its seizure type. Our initial experiments on platelets from epileptic patients suggested that peripheral benzodiazepine receptors of platelets could be a novel and effective therapeutic target.

\section{Acknowledgement}

The authors' great fully acknowledge the kind support of Higher Education Commission Pakistan as a part of HEJ strengthening grant Phase II.

\section{References}

Adam K. , W. Xiao-Jing, L. John, Bursting neurons signal input slope, The Journal of Neuroscience 22 (2002) 9053-9062.

Anthony A.G., S.B. Benjamin, The control of firing pattern in nigral dopamine neurons:burst firing, The Journal of Neuroscience 4 (1984) 2877-2890.

Avoli, M., Rogawski, M. A. \& Avanzini, G. Generalized epileptic disorders: an update. Epilepsia 42, 445-457 (2001).

Berkovic, S. F. in Epilepsy: a Comprehensive Textbook (eds Engel, J. Jr \& Pedley, T. A.) 217-224 (Lippincott-Raven,Philadelphia, 1998)

Bibiane F., C. Li-Qiong, S. Wolfgang, G.K. Roland, Modulation of the human cardiac sodium channel ac-subunit by cAMP-dependent protein kinase and the responsible sequence domain, Journal of Physiology 498 (1997) 309-318.

Carl E.S., Persistent sodium current and Its role in epilepsy, Current review in basic science 7 (2007) 15-22.

Charles E. Begley, Gus A. Baker, Ettore Beghi, James Butler, Daniel Chisholm, John T. Langfitt, Pierre Levy, Christopher Pachlatko, Samuel Wiebe and Karen Lee Donaldson (2007) Epilepsia Volume 48, Issue 5, pages 990-1001.

Chuan-ju L, D.D. Sulayman, R. Muthukrishnan, R.C. Theodore, G.W. Stephen, Modulation of the cardiac sodium channel Nav1.5 by fibroblast growth factor homologous factor 1B, The Journal of Biological Chemistry 278 (2003) 1029-1036.

Crawford, I.L. C.J. D, Localization and Release of Glutamic Acid in Relation to the Hippocampal Mossy Fibre Pathway, Nature (1973) 442-443.

David M.D., T.A. Dennis, O.M. Maxine, V.L. Darrell, Interneurons of the Dentate-Hilus Border of the Rat Dentate Gyrus: Morphological and Electrophysiological Heterogeneity, The Journal of Neuroscience 17 (1997) 3990-4005. 
De Lorenzo RJ, Sombati S, Coulter DA Effects of topiramate on sustained repetitive firing and spontaneous recurrent seizure discharges in cultured hippocampal neurons Epilepsia. 2000;41 Suppl 1:S40-4

Dietrich D, H. Clusmann, T. Kral, C. Steinhauser, I. Blumcke, U. Heinemann, J. Schramm, Two electrophysiologically distinct types of granule cells in epileptic human hippocampus, Neuroscience 90 (1999) 1197-1206.

Earl, P.D. I.L. L., C.W. A, G.A. L, The adult rat brain $\beta 1$ subunit modifies activation and inactivation gating of multiple sodium channel a subunits, The Journal of Biological Chemistry 269 (1994) 17649-17655.

Fatima Shad Kaneez and Sheikh Arshad Saeed (2007) Levels of serotonin and its metabolites in cultured neurons and platelets and their role in platelet aggregation. Experimental Brain Research Volume 183, Number 3 411-416.

Fatima Shad Kaneez Sheikh Arshad Saeed (2009) Investigating GABA and its function in platelets as compared to neurons. Platelets, 20, Issue 5328 - 333

Fatima Shad, Kaneez. (2006), Effect of D-serine on the serotonin receptors of human platelets.' Experimental Brain Research 173 (2) 353-356

Fatima SK, B. P.H, Morphological and electrical characteristics of postnatal hippocampal neurons in culture: the presence of bicuculline- and strychnine-sensitive IPSPs, Tissue \& Cell 30 (1998) 236-250.

Fatima-Shad, K. \& Barry, P. H. (1995) Heterogeneous current responses to GABA and glycine are present in post-natal cultured hippocampal neurons. Brain Res., 704 (2), 246-255.

Fatima-Shad, K. \& Barry, P. H.(1992) A patch-clamp study of GABA- and strychninesensitive glycine-activated currents in post-natal tissue-cultured hippocampal neurons. Proc. R. Soc. Lond. B., 250, 99-105.

Frank A.W., B.R. Mark, B. Maria, C. Jie, L. Huai-Ping, M. Grace, H.W. Joseph, M.I. Karen, S.W. Brian, T.S. James, R.J. Kenneth, Modulation of A-type potassium channels by a family of calcium sensors, Nature 403 (2000) 553-556

Hailing S., A. Gil, D.K. Eilon, Y. Yoel, Extracellular Calcium Modulates Persistent Sodium Current-Dependent Burst-Firing in Hippocampal Pyramidal Neurons, The Journal of Neuroscience 21 (2001) 4173-4182.

Jian M, Z. Shou-Yuan, K.M. Todd, H.E. Robert, D.A. Sam, Cannabinoid receptors differentially modulate potassium A and D currents in hippocampal neurons in culture, The Journal of Pharmacology and Experimental Therapeutics 291 (1999)

Joshua B.C., N.G. Lionel, M.A. David, Ionic mechanisms underlying repetitive high frequency burst firing in supragranular cortical neurons, The Journal of Neuroscience, July 20 (2000) 4829-4843.

Knopfel T., B.H. Gahwiler, Activity-induced elevations of intracellular calcium concentration in pyramidal and nonpyramidal cells of the CA3 region of rat hippocampal slice cultures, Journal of Neurophysiology 68 (1992) 961-963.

Kocsis, J.D. and Mattson, R.H., 1996. , GABA levels in the brain: a target for new antiepileptic drugs. Neuroscientist 2, pp. 326-334

Kristopher M.K., N.M. Sunita, L.G.J. Alfred, Impaired inactivation gate stabilization predicts increased persistent current for an epilepsy-associated SCN1A mutation, The Journal of Neuroscience 26 (2006) 10958 -10966.

LTan M., H.P. Theeuwes, L. Feenstra, J.G.G. Borst, Membrane Properties and Firing 
Mark B, F. Debra, H.C. Todd, L.B. Irwin, Modulation of the Kv1.3 potassium channel by receptor tyrosine kinases, J. Gen. Physiol. 110 (1997).

Methvin Isaac (2005) Serotonergic 5-HT2C Receptors as a Potential Therapeutic Target for the Design Antiepileptic Drugs. Current Topics in Medicinal Chemistry 2005, 5, 59-67

Michael Stegen, zChristina C. Young, Carola A. Haas, Josef Zentner, and Jakob Wolfart (2009) Increased leak conductance in dentate gyrus granule cells of temporal lobe epilepsy patients with Ammon's horn sclerosis. Epilepsia, 50(4):646-653.

Nikitin E.S., T. Kiss, K. Staras, M. O'Shea, P.R. Benjamin, G. Kemenes, Persistent Sodium Current Is a Target for cAMP-Induced Neuronal Plasticity in a State-Setting Modulatory Interneuron, J Neurophysiol 95 (2006) 453-463.

Patterns of Inferior Colliculus Neurons: An In Vivo Patch-Clamp Study in Rodents, J Neurophysiol 98 (2007) 443-453.

Peter H., E. Daniel, B. Angela, M. Michele, A.A. Giorgio, B.J. David, Distinct classes of pyramidal cells exhibit mutually exclusive firing patterns in hippocampal area CA3b, Hippocampus 18 (2008) 411-424.

Podlogar M., D. Dirk, Firing pattern of rat hippocampal neurons: A perforated patch clamp study, Brain research 1085 (2006) 95-101.

Robert B., C. Qing, V. Alex, T. Glenn, N. Jeffrey, A. Richard, BK channel $\beta 4$ subunit reduces dentate gyrus excitability and protects against temporal lobe seizures, Nature Neuroscience 8 (2005) 1752-1759.

Smith M., J. Perrier, Intrinsic properties shape the firing pattern of ventral horn interneurons from the spinal cord of the adult turtle, J Neurophysiol 96 (2006) 2670-2677.

Steinlein, O. K. \& Noebels, J. L. Ion channels and epilepsy in man and mouse. Curr. Opin. Genet. Dev. 10, 286-291 (2000).

Young CC, Stegen M, Bernard R, Müller M, Bischofberger J, Veh RW, Haas CA, Wolfart J (2009) Up regulation of inward rectifier K+ (Kir2) channDeLorenzo RJ, Sombati S, Coulter DA (2000els in dentate gyrus granule cells in temporal lobe epilepsy J Physiol.;587(Pt 17):4213-33.

Yunru L., A.G. Monica, J.B. David, Role of Persistent Sodium and Calcium Currents in Motoneuron Firing and Spasticity in Chronic Spinal Rats, J Neurophysiol 91 (2004) 767-783.

Zaid Afawi, Arvid Suls, Dana Ekstein, Sara Kivity, Miriam Y Neufeld, Karen Oliver, Peter De Jonghe, Amos D Korczyn, Samuel F. Berkovic Mild adolescent/adult onset epilepsy and paroxysmal exercise-induced dyskinesia due to GLUT1 deficiency Epilepsia Volume 51, Issue 12, pages 2466-2469, December 2010

Zhi-Jiang H., S. Xue-Jun, Differing alterations of sodium currents in small dorsal root ganglion neurons after ganglion compression and peripheral nerve injury, Molecular Pain 4 (2008). 


\title{
Epilepsy: Selenium and Aging
}

\author{
Caroline Rocourt, Ying Yu and Wen-Hsing Cheng \\ Department of Nutrition and Food Science, University of Maryland, College Park
}

USA

\section{Introduction}

Epilepsy affects nearly 50 million people worldwide and is a disease that involves spontaneous frequent seizures caused by electrical disturbances in the brain (Bergen 1998; Meinardi, Scott et al. 2001). Epilepsy is a syndrome, or a collection of symptoms, in which people are predisposed to seizures (Fisher, van Emde Boas et al. 2005; Gomez-Alonso and Giraldez 2007; Jaseja 2009). Epilepsy diagnosis usually occurs after permanent injury to the brain or from inborn errors that cause brain tissue to become abnormally excited (Fisher, van Emde Boas et al. 2005). Health statistics show that children less than 2 and adults older than 65 are the most likely populations to develop epilepsy and that seizure events increase the risk of mortality in the individual (Feng 1978; Tsai 2005; Fountain, Van Ness et al. 2011). It is thought that nearly $4 \%$ of the US population will have seizure activity in their lifetimes, with many having seizures and never being aware they occurred.

In some types of epilepsy, the cause of the seizures cannot be clearly defined and does not present with other neurological disorders (Lerman, Sagie et al. 2011). Certain types of epilepsy have a genetic component (Poduri and Lowenstein 2011; Sisodiya and Mefford 2011; Sozmen, Baybas et al. 2011; Wilke, Worrell et al. 2011), but the most common diagnoses of epilepsy occur after neurological damage such as a stroke, transient ischemic attacks, a diagnosis of dementia, traumatic brain injury, brain abscesses, meningitis, encephalitis, neurosyphilis, brain tumors, hematomas, abnormal blood vessels, AIDS, congenital brain defects, metabolic diseases such as phenylketonuria, and liver or kidney failure.

Epileptic seizures range in severity from staring spells to violent convulsions and loss of consciousness (Haut, Lipton et al. 2005; Maillard, Vignal et al. 2009). Given the wide variety of factors that can precipitate epilepsy, it stands to reason that type of seizure is dependent on the location and type of the brain injury. Upon physical examination, many people suffering from epilepsy appear healthy (Maganti, Gerber et al. 2008). Most epileptics will present with clinical abnormal electrical activity as measured by an electroencephalograph (Urbach 2005). Epilepsy is further classified as idiopathic (presumed genetic basis), symptomatic (due to a structural abnormality) or cryptogenic (cause is undetermined) (Kwan and Brodie 2000; Berg and Kelly 2006; Farrell, Wirrell et al. 2006).

Unfortunately, after some epileptic events, there is a risk of neuron destruction. This is especially concerning due to the limited capacity of the brain to regenerate neurons and oftentimes, the damage is permanent. Following a seizure, epileptic's brains often form excitotoxic lesions which destroy neurons by initiating apoptosis. The current treatment for epilepsy is limited to controlling the seizure activity. Anticonvulsants are oral medications used to prevent seizures in patients with epilepsy. The specific drug prescribed to patients 
varies based on their past seizure history and any underlying conditions that may have contributed to the epileptic diagnosis. (Prunetti and Perucca 2011; Steinert, Baier et al. 2011; Strzelczyk, Cenusa et al. 2011; Verrotti, Loiacono et al. 2011; Yasuda, Sugiura et al. 2011). In patients with epilepsy resulting from chronic infections, the epileptic symptoms will subside after the course of drug treatment. Also, epilepsy occurring after tumor development or following injury to blood vessels, such as a hematoma, can be successfully managed in some cases after the underlying cause of injury is treated. Nevertheless, it is difficult to treat these types of brain injuries; so many patients use proper medication to prevent the seizures (Sander 1993).

The cases of epilepsy that do not respond to medication (Go and Snead 2008; Berg 2009; Nakken and Tauboll 2009), are often candidates for invasive and risky procedures such as brain surgery. Surgery can remove the damaged cells of the brain which cause the abnormal electrical signals. Other surgical procedures such as implantation of a device, similar to a heart pacemaker, stimulate the vagal nerve and help reduce the frequency of seizures (Landre 2004). Unfortunately, there are also types of epilepsy that cannot be managed well with medication or any other available therapies and the general term for this type of epilepsy is called refractory or intractable epilepsy (Sander 1993; Kwan and Brodie 2000; Lerman, Sagie et al. 2011). Growing lines of evidence have indicated a role of inflammation in causing and exacerbating intractable epilepsy (Vezzani, French et al. 2011). Patients with intractable epilepsy often go on specialized diets which are thought to help reduce the severity and number of seizures. Popular diet choices for epileptic patients are diets low in carbohydrates, such as the ketogenic diet (Kossoff and McGrogan 2005), which is very low in carbohydrates and very high in fat but still calorie balanced for the individual. For undetermined reasons, when the body of an epileptic burns fat for fuel, rather than glucose, it can help control and prevent seizures (Baranano and Hartman 2008; Choragiewicz, Zarnowska et al. 2010; Westmark, Westmark et al. 2010). While the ketogenic diet is effective, long term health implications prevent patients from being on the diet for periods longer than two years (de Kinderen, Lambrechts et al. 2011). Dietary antioxidants are known to protect against seizures possibly by increasing the free radical scavenging capabilities of key antioxidant enzymes in the brain (Garjani, Fathiazad et al. 2009; Mehla, Reeta et al. 2010; Militao, Ferreira et al. 2010; Pages, Maurois et al. 2010; Sharma, Nehru et al. 2010; Tome Ada, Ferreira et al. 2010). Using a nutrigenomics approach to identify compounds with a beneficial effect (Suzuki 2011), researchers can identify genes associated with epilepsy susceptibility and then test whether particular dietary components, in this case selenium, can be used to improve the incidence, frequency, and severity of epilepsy and the resulting complications.

\section{Selenium and selenoproteins}

Selenium is a non-metal trace element discovered in 1817 as a by-product of sulfuric acid production (Brown and Arthur 2001). Selenium exists in nature in organic (such as selenomethionine and selenocysteine) or inorganic (such as selenate and selenite) forms (Puzanowska-Tarasiewicz, Kuzmicka et al. 2009).

Selenium can be incorporated non-specifically into methionone-rich proteins, such as those in the Brazil nut and sunflower seeds (Kortt, Caldwell et al. 1991). The biological functions of selenium are mainly mediated by selenoproteins, which contain selenocysteine (Allmang, Wurth et al. 2009; Lu and Holmgren 2009). Selenocysteine (Sec) has its own tRNA and its own 
codon, UGA (de Jesus, Hoffmann et al. 2006). Sec is inserted into mRNA in response to UGA codons in the genome of all selenoproteins. Selenoprotein mRNA is characterized by selenocysteine insertion sequence element (SECIS) (Howard, Moyle et al. 2007; Mix, Lobanov et al. 2007), and a RNA-binding protein complex (Bock, Forchhammer et al. 1991; SmallHoward and Berry 2005; Allmang and Krol 2006; Squires and Berry 2008). Some seleniumcontaining proteins such as the liver protein $56 \mathrm{~K}$ and the liver fatty acid binding protein (Behne, Weiss-Nowak et al. 1994) can sequester Se, but they do not contain selenocysteine.

Selenium was only recognized as a toxicant (Belogorsky and Slaughter 1949; Fels and Cheldelin 1949; Klug, Harshfield et al. 1952; Mc and Portman 1952; Fabre and Truhaut 1956) and its biological role was unknown until 1952 when its essential role was discovered in microorganisms. It is a trace mineral that is essential for humans (Papp, Lu et al. 2007), which was first recognized when when dietary supplementation of selenium prevented liver necrosis in rats efficiently (Mertz and Schwarz 1958; Schwarz, Stesney et al. 1959; Schwarz, Porter et al. 1972). Following these studies, it was found that selenium was essential for glutathione peroxidase (GPX) activity (Flohe, Gunzler et al. 1973; Rotruck, Pope et al. 1973). Sec, now identified as the $21^{\text {st }}$ amino acid was indentified in eubacteria and archaebacteria model systems (Ambrogelly, Palioura et al. 2007; Xu, Carlson et al. 2007). Stadtman and colleagues found that selenium was incorporated into proteins in the form of selenocysteine through labeled Clostridium sticklandii with ${ }^{75}$ Se metabolically (Cone, Del Rio et al. 1976; Axley and Stadtman 1989; Lee, Worland et al. 1989; Leinfelder, Stadtman et al. 1989; Stadtman, Davis et al. 1989). UGA, the stop codon, was discovered for selenocysteine incorporation in murine and GPX1 gene was cloned at the same time (Chambers, Frampton et al. 1986).

Selenium deficiency (Kakturskii, Strochkova et al. 1990), or Keshan disease, is responsible for the cardiomyopathy in children living in parts of China where the soil lacks selenium (Guanqing 1979; Yang and Xia 1995; Yang, Chen et al. 2007). Recently, the gene of Secisbp2 protein, which is related with synthesis of selenoproteins (Copeland and Driscoll 1999; Copeland, Fletcher et al. 2000; Low, Grundner-Culemann et al. 2000; Tujebajeva, Copeland et al. 2000; Berry, Tujebajeva et al. 2001; Copeland and Driscoll 2001; Copeland, Stepanik et al. 2001; Fletcher, Copeland et al. 2001; Copeland and Driscoll 2002; Lescure, Allmang et al. 2002; Lescure, Fagegaltier et al. 2002), has been studied as a possible target for hereditary diseases, an example of which includes human thyroid hormone metabolism disorder (Dumitrescu, Liao et al. 2005). Selenium is also known as an important chemoprevention agent (El-Sayed, Aboul-Fadl et al. 2006; Micke, Schomburg et al. 2009) by inducing apoptosis in cancer cells (Jackson and Combs 2008) and senescence in the early stage of tumorigenesis (Wu, Kang et al. 2010) in a dose-dependent manner.

More than 50 years ago, the ROS (reactive oxygen species) theory of aging was proposed. However, there is still there is a lack of irrefutable evidence in support or opposition of this theory. ROS can be induced by exogenous sources, such as UV or ionizing irradiation (Finkel 2000), which are by-products of mitochondrial respiration and widespread in vivo as the form of superoxides, hydroxyl free radicals, and hydrogen peroxides (Harman, 1956). As previous studies have shown, GPX1 contributes much of the $\mathrm{H}_{2} \mathrm{O}_{2}$-eliminating activity of selenium (Cheng, Ho et al. 1997; Cheng, Ho et al. 1997; Lei, Cheng et al. 2007). Therefore, it is logical to address the ROS theory of aging through investigation of selenium and selenoproteins (Hawkes and Alkan 2010). Some studies have implied that the accumulation of lipid oxidation in mitochondria has the potential to suppress the increased activity of GPX when comparing aged and juvenile rats (Nohl, Hegner et al. 1979). However, there is 
no conclusive evidence to identify the biological function of GPX1 in lifespan extension in mouse models (Ladiges, Van Remmen et al. 2009). Interestingly, GPX4-/- mice are embryonic lethal while GPX4+/- mice displays lifespan extension (Ran, Liang et al. 2007).

Most of the selenoproteins that have been identified are enzymes with selenium in their active sites. Through scanning the whole human genome to find SECIS, a total of 25 selenoproteins were discovered (Kryukov, Castellano et al. 2003), which contain five glutathione peroxidases (GPX1-4, GPX6), three thioredoxin reductases (TR1-3) (Hondal and Ruggles 2010), three iodothyronine deiodinases (DI-III), the 15-kDa selenoprotein (Sep 15), selenophosphate synthetase-2 (SPS2), and selenoprotein H, I, K, M, N, O, P, R, S, T, U, V. All selenoproteins only have one selenocysteine residue except for selenoprotein $\mathrm{P}$ which contains 10 residues (Burk and Hill 1999). The domains containing selenocysteine residues in selenoprotein are highly related with their enzyme activities (Hill, Zhou et al. 2007). It was noted that cysteine replacing the selenocysteine residue in selenoproteins lead to reduction of catalytic activity (Hazebrouck, Camoin et al. 2000; Lee, Bar-Noy et al. 2000; Korotkov, Novoselov et al. 2002; Kryukov and Gladyshev 2002).

Notably, more than one-third of selenoproteins play a critical role in combating oxidative stress. The GPX family uses glutathione as a reducing equivalent to eliminate hydrogen peroxide, organic hydroperoxides and phospholipid hydroperoxides (Bosch-Morell, Flohe et al. 1999; Flohe, Hecht et al. 1999). The redox status of thioredoxin is under the control of the thioredoxin reductase (TR) family (Bjornstedt, Hamberg et al. 1995; Holmgren and Bjornstedt 1995). Sel P is thought to be not only a selenium carrier, which distributes in body fluids, but capable of eliminating phospholipid hydroperoxides (Saito, Hayashi et al. 1999; Takebe, Yarimizu et al. 2002). It is important to elucidate the mechanism by which selenoperoxidases eliminate ROS in the brain and to identify additional selenoperoxidases and their functions during aging and neuron degeneration.

\section{Selenium and selenoproteins in epilepsy}

The brain expresses most selenoproteins and is at the apex of selenium retention in the body (Nakayama, Hill et al. 2007). It is proposed that selenium may be necessary for prevention of epilepsy or other degenerative neurological disorders; however, there is no consensus in the field whether selenium plays a direct role or not. Selenium has a role in protection the neurons from excitotoxic insults, such as the continuous stimulation of a nerve cell by glutamate or another neurotransmitter, and thus can decrease the insult burden on the neurons (Savaskan, Brauer et al. 2003).

Although no severe neurological phenotypes have been associated with a selenium deficient diet, this may be due to the brain's preferential sequestration of body selenium. Mice on a selenium deficient diet are, however, more susceptible to neuropathological changes (Hill, Zhou et al. 2004). It is not well known how selenium status in the blood and the brain are correlated (Chen and Berry 2003), but it is known that selenium supplementation can prevent dopamine loss and degeneration of neurons in the substantia nigra, and reduce lipid peroxidation (Aldeeb, Almoutaery et al. 1995; Imam, Newport et al. 1999; Zafar, Siddiqui et al. 2003). Selenium was shown to protect the neurons through selenoproteins (Savaskan, Brauer et al. 2002; Lamarche, Signorini-Allibe et al. 2004; Reeves, Bellinger et al. 2010; Wang, Geng et al. 2010) and other studies that combine selenium treatment with anticonvulsant medication showed a synergistic protective effect against induced seizures (Kutluhan, Naziroglu et al. 2009). Also, in cases of selenium deficiency, neurons are exposed 
to increased glutamate-induced excitotoxicity because selenium has an inhibitory effect on the glutamate induced NF- $\mathrm{KB}$ and AP-1 activation (Savaskan, Brauer et al. 2002; Santamaria, Vazquez-Roman et al. 2005).

There are a total of 24 selenoproteins in mice, all of which are expressed in the brain, particularly in neurons of the olfactory bulb, hippocampus, cerebral cortex and cerebellar cortex (Zhang, Zhou et al. 2008). In these tissues Gpx4, Sel K, Sel M, Sel W, and Sel 15 mRNAs were expressed the most, but in the choroid plexus over half of the identified selenoproteins were expressed. Gpx4, Sel P, and Sel W genes were highly expressed in over $90 \%$ of the brain, similar to findings from previous studies that found Sel P and Sel W were highly expressed in the rodent brain (Dreher, Schmutzler et al. 1997; Steinert, Bachner et al. 1998; Sun, Butler et al. 2001; Hoffmann, Hoge et al. 2007; Nakayama, Hill et al. 2007; Renko, Hofmann et al. 2009).

There are some regions of the rodent brain that express significantly lower levels of selenoproteins, which implies that some structures of the brain are less dependent on selenium availability. Selenoprotein genes are expressed the lowest in the oculomotor nucleus, Edinger-Westphal nucleus, nucleus Raphé pontis, anteroventral periventricular nucleus and dorsal premammillary nucleus; however, these brain regions do express the same selenoproteins that are found to be highly expressed in other regions of the brain, but at a significantly lower level, such as Sel P, and Sel W and Gpx 4 (Zhang, Zhou et al. 2008). In the white matter of the brain, or corpus callosum, selenoprotein expression is considerably less than in other regions of the brain. The corpus collosum consists of mainly myelinated axons and is responsible for carrying the nerve impulses to different regions of gray matter. Generation of ROS and the resulting oxidative stress are known to be both the cause and consequence of many neurodegenerative conditions (Sander 1993). Post mortem autopsies of people with the neurodegenerative disorders Parkinson's disease, Alzheimer's disease, and amyotrophic lateral sclerosis all show that there are increased ROS in the regions of the brains that were affected by the neurodegenerative disorder. The brain has a reduced capacity for regeneration and a high metabolic rate which predisposes it to oxidative damage. The repair mechanisms that the cells have employed to counteract oxidative damage are primarily antioxidant enzymes such as glutathione peroxidases and thioredoxin reductases which are dependent on selenium for their function. Accumulating lines of evidence suggest that selenium-dependent antioxidant enzymes and selenoproteins are integral to epilepsy and have a role in the progression of the disease. It is promising to use selenium as a line of treatment against degenerative free radical diseases such as epilepsy.

\subsection{The Se-dependent glutathione peroxidase}

The function of the GPX family is to remove hydrogen and other peroxides from the brain and other tissues by coupling its reduction to water and alcohols using glutathione as a reducing equivalent. Glutathione is found in the mitochondria and cytoplasm of brain tissue and has been found to be released into the extracellular space by astrocytes. Interestingly, the first report of selenium status affecting any neurological condition was in infants with intractable epilepsy (Weber, Maertens et al. 1991). Infant brains are particularly susceptible to oxidative damage, which can contribute to abnormal brain electrical signaling, because of the high concentration of unsaturated fatty acids in the infant brain needed to synthesize cholesterol and fatty acids. These nutrients are necessary for the billions of developing nerve connections. 
The seizure symptoms of these infants can be reversed with selenium supplementation (Weber, Maertens et al. 1991; Ramaekers, Calomme et al. 1994). Children suffering from intractable epilepsy are deficient in glutathione peroxidase activity. From the four children studied, two had normal blood selenium and high concentrations of GPX in their plasma, but low enzymatic activity. The other children had low intracellular selenium levels and low GPX expression and activity. Selenium supplementation and discontinuation of their anticonvulsant medication decreased seizure activity. The second study found that oral selenium supplements in children $(3-5 \mu \mathrm{g} / \mathrm{kg}$ body weight) decreased frequency of seizures, improved electroencephalograph recordings, and restored liver function in the infants after two weeks of treatment (Ramaekers, Calomme et al. 1994). It was thought that supplementation of selenium restored the function of selenium-dependent GPX1 and GPX4 activity. From these results, selenium status is an important triggering factor for the onset of intractable seizures and that selenium-conferred antioxidant protection in the brain can help prevent neuronal damage following frequent seizures.

It has been suggested that treatment with anticonvulsants, such as valproic acid, which induces oxidative stress, depletes total body selenium and decreases GPX activity (Naziroglu 2009). However, others believe that selenium supplementation in congruence with antiepileptic drugs increase the risk of systemic toxicity. The conflicting selenium levels are likely associated with the pharmacology of the particular drug, duration of treatment, age of subject, validity of plasma selenium estimating brain selenium concentrations, and the underlying cause of the patient's epilepsy. It has been found that epileptic patients, without medication, have lower blood and tissue levels of selenium in addition to lower levels of selenoproteins and lower levels of selenium-dependent redox enzymes. In particular, intractable epileptics have been shown to have significantly lower mean serum selenium levels compared to a control group (Ashrafi, Shabanian et al. 2007). Animal models have shown that selenium can prevent the development of iron-induced epileptic symptoms due to selenium's ability to combat peroxidative injury (Rubin and Willmore 1980; Willmore and Rubin 1981).

\subsection{Thioredoxin reductases}

Thioredoxin reductases (TR) use NADPH for reduction of thioredoxin in various cellular redox pathways (Tamura and Stadtman 2002). TR-3 is expressed in very low levels in the mouse brain (Zhang, Zhou et al. 2008). Although TR-1 is well characterized and expressed in high levels in other organs, it is not highly expressed in the brain. Since TR is important for intracellular redox regulation and antioxidant defense (Schweizer, Brauer et al. 2004), decreased TR expression and activities could lead to enhanced cell loss, thus increasing the risk for epilepsy and other neurodegenerative conditions.

\subsection{Selenoprotein $\mathbf{P}$}

Selenoprotein P (Sel P) is unique because it contains 10-17 selenocysteine residues, unlike other selenoproteins which contain only one. Sel P is produced mostly in the liver, but all tissues make and secrete Sel P into the plasma (Burk and Hill 2005; Hoffmann, Hoge et al. 2007). In rodents, Sel P is a predominant form of selenium in the plasma. Sel P is responsible for selenium transportation throughout the body, particularly to the brain where selenium is needed for incorporation into other selenoproteins which confer antioxidant benefits (Hill, Zhou et al. 2007). Sel P knockout mice develop seizures and movement disorders when raised on selenium restricted diets. 
During seizure activity in epileptics, the neurons in the affected areas of the brain often have redox shifts due to the large influx of calcium through voltage gated channels (Stefani, Spadoni et al. 1997). If cellular redox equilibrium is not quickly restored following seizures, it is probable that the neurons will die and that region of the brain will be unable to function properly (Wirth, Conrad et al. 2010). It is thought that selenium, probably through its function in the GPX or TR families, can help prevent neuronal cell death and neutralize ROS which is generated as a consequence of high intracellular calcium (Xiong, Markesbery et al. 2007). Typically the brain can handle redox shifts and occasional electrolyte imbalances; however, during a seizure these activities happen at a rate and frequency that the cells cannot handle (Howse and Duffy 1975). The neurons eventually will die, and cause an increase in potentially harmful cellular byproducts (Barinaga 1998).

\subsection{Selenoprotein $\mathrm{W}$}

Selenoprotein W (Sel W) has a redox motif and binds glutathione (Beilstein, Vendeland et al. 1996; Whanger 2009), similar to the well characterized GPX family. The ' $W$ ' designation is because of the white muscle disease seen in grazing livestock in areas where the soil is depleted of selenium (Vendeland, Beilstein et al. 1993). Sel W is highly expressed in the four basic brain regions in rodents; the hippocampus, olfactory area, cerebellar cortex, and isocortex (Zhang, Zhou et al. 2008).

The expression of Sel W is induced in the neurons of mesial temporal lobe epilepsy patients more than tenfold and is associated with BCL2 expression, which suggests that the Sel W induction is a defensive response to oxidative stress (Yuzbasioglu, Karatas et al. 2009). Furthermore, the expression of Sel W in cortex, cerebellum, and thalamus remains constant under Se deficiency (Sun et al., 2001b). Thus, Sel W expression is maintained in certain regions of the brain under selenium deficiency, suggesting a critical role of Sel $W$ in the protection against seizure activities that is associated with increased oxidative stress.

\subsection{Selenoprotein $\mathbf{H}$}

Selenoprotein H (Sel H) is a nucleolar DNA-binding protein (Novoselov, Kryukov et al. 2007) and may function as a transcription factor (Panee, Stoytcheva et al. 2007). It has been found that Sel H mRNA is highly expressed in the hippocampus during development but level falls quickly after birth and is undetectable in the adult brain (Zhang, Zhou et al. 2008); however, in another study the Sel H gene was found to be expressed in the adult mouse brain by using the RT-PCR method (Hoffmann, Hoge et al. 2007).

\subsection{Selenoprotein M}

Selenoprotein $\mathrm{M}(\mathrm{Sel} \mathrm{M})$ is a small endoplasmic reticulum protein with unknown function, which is highly expressed in cornu ammonis granule cells of dentate gyrus (Zhang, Zhou et al. 2008). Cornu ammonis is also known as Ammon's horn and is part of the interlocking gyri which makes up the hippocampus. Interestingly, the most common form of neuropathological damage in temporal lobe epilepsy patients is sclerosis (hardening of tissue) of the cornu ammonis, also known as Ammon's horn sclerosis, which is characterized by neuronal cell loss in the hippocampus region of the brain. Common clinical markers of the disease include granule cell dispersion, reactive gliosis, and the segmental loss of pyramidal neurons. Unfortunately, $65 \%$ of people with temporal lobe epilepsy suffer from this disease (Blumcke, Thom et al. 2002; de Lanerolle and Lee 2005). Many of the seizures 
due to temporal lobe epilepsy are very poorly controlled with anticonvulsant drugs. The role of Sel M is not fully understood and it is unclear whether Ammon's horn sclerosis is the cause of seizures of epileptics, or rather damage from the ongoing seizure activity.

\section{Selenium and neurological disorders}

Most inherited or congenital forms of epilepsy develop and occur before the age of 2. Older adults who have developed epilepsy have done so mostly because of brain injuries (hematomas and other bleeding vessels) or after a stroke. Generally, the most common cause of epilepsy in the elderly is attributed to cerebrovascular diseases or prescription drug reactions. Older populations are more at risk for drugs causing epileptiform activities because of their increased sensitivity to all drugs due to decreased drug metabolism.

Also other diseases in the elderly, particularly those that cause impaired language function or overall neurological deterioration, have been associated with increased seizure risks. Other factors such as metabolic problems, uraemia, thyroid problems, low blood sugar, electrolyte imbalances and liver damage can all contribute to seizures in the older population. In addition to the metabolic risk factors, epilepsy risk can also increase in patients with neurological disorders such as Alzheimer's (Noebels 2011), down syndrome, and dementia (Ito, Echizenya et al. 2009; Palop and Mucke 2009; Rao, Dove et al. 2009; Scarmeas, Honig et al. 2009; Larner 2010; Westmark, Westmark et al. 2010).

There is a link between neurological disorders and aging (Zhang, Rocourt et al. 2010). Since the body preferentially retains selenium in the brain even during periods of selenium deficiency, which further implies that selenium plays an important role in the brain (Behne, Hilmert et al. 1988; Chen and Berry 2003). Neurological disorders such as Alzheimer's disease, Parkinson's disease, and those disease states resulting from damage caused by environmental toxins, ischemic insults, drug abuse, and brain tumors are exacerbated by ROS (Chen and Berry 2003).

The selenium protection against neurological disorders is primarily conferred through antioxidative selenoproteins (Zhang, Rocourt et al. 2010). Therefore, selenoproteins play an important role in regulating redox reactions in the body, and particularly the brain. For example, the brains in mouse models of Alzheimer's disease show reduced levels of Sel M, which could mean that this selenoprotein plays a protective role in the development of Alzheimer's disease (Hwang, Cho et al. 2005).

Sel $\mathrm{P}$ knockout mice have movement disorders and seizures when given selenium-restricted diets from their birth (Hill, Zhou et al. 2003; Schomburg, Schweizer et al. 2003; Kutluhan, Naziroglu et al. 2009). Rats on selenium deficient diets have an increased risk of seizures and neuronal loss, further providing evidence that selenium can help prevent seizures. Because epilepsy is characterized by neuronal loss resulting from pro-apoptotic factors in association with oxidative stress (Savaskan, Brauer et al. 2003), selenium might help alleviate the underlying causes of brain injury that precipitate seizures.

\section{Nutrigenomics perspectives}

There is much evidence that speculates on the genetic aspect of epilepsy (Rees 2010; Sisodiya and Mefford 2011). Nonetheless, since epilepsy is a syndrome and not a disease, the cause of seizures in epileptics varies widely. Furthermore, even if epilepsy could be divided into groups based on the respective, underlying cause, it would still be difficult to determine the 
genetic influence because of changes across clinical subgroups and amongst families. However, some types of epilepsy have been shown to have strong genetic components (Greenberg and Subaran 2011): 1) partial epilepsy has been linked to chromosome $10 \mathrm{q} ; 2$ ) benign familial neonatal convulsions have been mapped to chromosome $20 \mathrm{q}$ and another locus found on $8 \mathrm{q}$; 3) a progressive form of myoclonus epilepsy has been localized to chromosome $21 \mathrm{q}$; 4) juvenile myoclonic epilepsy has been associated with regions of chromosome 6. Interestingly, the types of epilepsy syndromes that have genetic linkage evidence are a very small proportion of all epilepsy cases and no genetic basis has been found for most types of epilepsy.

Nutrigenomics is the study of how nutrition impacts the variation of gene expression across individuals. Seeing as how genetically diverse individuals are, it would stand to reason that individualized diets based on a person's genetic background can contribute to better overall health. Nutrigenomic approaches will become increasingly necessary in treating chronic diseases, including epilepsy. In the future it is expected that we will uncover genetic evidence that explains why certain people have different responses to similar diet, and how such variations help alleviate neuronal disorders.

\section{References}

Aldeeb, S., K. Almoutaery, et al. (1995). "Neuroprotective Effect of Selenium on Iminodipropionitrile-Induced Toxicity." Journal of Psychiatry \& Neuroscience 20(3): 189-192.

Allmang, C. and A. Krol (2006). "Selenoprotein synthesis: UGA does not end the story." Biochimie 88(11): 1561-71.

Allmang, C., L. Wurth, et al. (2009). "The selenium to selenoprotein pathway in eukaryotes: more molecular partners than anticipated." Biochim Biophys Acta 1790(11): 141523.

Ambrogelly, A., S. Palioura, et al. (2007). "Natural expansion of the genetic code." Nat Chem Biol 3(1): 29-35.

Ashrafi, M. R., R. Shabanian, et al. (2007). "Selenium and intractable epilepsy: Is there any correlation?" Pediatric Neurology 36(1): 25-29.

Axley, M. J. and T. C. Stadtman (1989). "Selenium metabolism and selenium-dependent enzymes in microorganisms." Annu Rev Nutr 9: 127-37.

Baranano, K. W. and A. L. Hartman (2008). "The ketogenic diet: uses in epilepsy and other neurologic illnesses." Curr Treat Options Neurol 10(6): 410-9.

Barinaga, M. (1998). "Stroke-damaged neurons may commit cellular suicide." Science 281(5381): 1302-3.

Behne, D., H. Hilmert, et al. (1988). "Evidence for specific selenium target tissues and new biologically important selenoproteins." Biochim Biophys Acta 966(1): 12-21.

Behne, D., C. Weiss-Nowak, et al. (1994). "Application of nuclear analytical methods in the investigation and identification of new selenoproteins." Biol Trace Elem Res 43-45: 287-97.

Beilstein, M. A., S. C. Vendeland, et al. (1996). "Selenoprotein W of rat muscle binds glutathione and an unknown small molecular weight moiety." J Inorg Biochem 61(2): 117-24.

Belogorsky, J. B. and D. Slaughter (1949). "Administration of BAL in selenium poisoning." Proc Soc Exp Biol Med 72(1): 196-8. 
Berg, A. T. (2009). "Identification of pharmacoresistant epilepsy." Neurol Clin 27(4): 1003-13.

Berg, A. T. and M. M. Kelly (2006). "Defining intractability: comparisons among published definitions." Epilepsia 47(2): 431-6.

Bergen, D. C. (1998). "Preventable neurological diseases worldwide." Neuroepidemiology 17(2): 67-73.

Berry, M. J., R. M. Tujebajeva, et al. (2001). "Selenocysteine incorporation directed from the 3'UTR: characterization of eukaryotic EFsec and mechanistic implications." Biofactors 14(1-4): 17-24.

Bjornstedt, M., M. Hamberg, et al. (1995). "Human thioredoxin reductase directly reduces lipid hydroperoxides by NADPH and selenocystine strongly stimulates the reaction via catalytically generated selenols." Journal of Biological Chemistry 270(20): 11761-4.

Blumcke, I., M. Thom, et al. (2002). "Ammon's horn sclerosis: a maldevelopmental disorder associated with temporal lobe epilepsy." Brain Pathol 12(2): 199-211.

Bock, A., K. Forchhammer, et al. (1991). "Selenocysteine: the 21st amino acid." Mol Microbiol 5(3): 515-20.

Bosch-Morell, F., L. Flohe, et al. (1999). "4-Hydroxynonenal inhibits glutathione peroxidase: protection by glutathione." Free Radic Biol Med 26(11-12): 1383-7.

Brown, K. M. and J. R. Arthur (2001). "Selenium, selenoproteins and human health: a review." Public Health Nutr 4(2B): 593-9.

Burk, R. F. and K. E. Hill (1999). "Orphan selenoproteins." Bioessays 21(3): 231-7.

Burk, R. F. and K. E. Hill (2005). "Selenoprotein P: an extracellular protein with unique physical characteristics and a role in selenium homeostasis." Annu Rev Nutr 25: 215-35.

Chambers, I., J. Frampton, et al. (1986). "The structure of the mouse glutathione peroxidase gene: the selenocysteine in the active site is encoded by the 'termination' codon, TGA." EMBO J 5(6): 1221-7.

Chen, J. and M. J. Berry (2003). "Selenium and selenoproteins in the brain and brain diseases." Journal of Neurochemistry 86(1): 1-12.

Cheng, W. H., Y. S. Ho, et al. (1997). "Overexpression of cellular glutathione peroxidase does not affect expression of plasma glutathione peroxidase or phospholipid hydroperoxide glutathione peroxidase in mice offered diets adequate or deficient in selenium." Journal of Nutrition 127(5): 675-80.

Cheng, W. H., Y. S. Ho, et al. (1997). "Cellular glutathione peroxidase knockout mice express normal levels of selenium-dependent plasma and phospholipid hydroperoxide glutathione peroxidases in various tissues." Journal of Nutrition 127(8): 1445-50.

Choragiewicz, T., I. Zarnowska, et al. (2010). "[Anticonvulsant and neuroprotective effects of the ketogenic diet]." Przegl Lek 67(3): 205-12.

Cone, J. E., R. M. Del Rio, et al. (1976). "Chemical characterization of the selenoprotein component of clostridial glycine reductase: identification of selenocysteine as the organoselenium moiety." Proc Natl Acad Sci U S A 73(8): 2659-63.

Copeland, P. R. and D. M. Driscoll (1999). "Purification, redox sensitivity, and RNA binding properties of SECIS-binding protein 2, a protein involved in selenoprotein biosynthesis." Journal of Biological Chemistry 274(36): 25447-54.

Copeland, P. R. and D. M. Driscoll (2001). "RNA binding proteins and selenocysteine." Biofactors 14(1-4): 11-6. 
Copeland, P. R. and D. M. Driscoll (2002). "Purification and analysis of selenocysteine insertion sequence-binding protein 2." Methods Enzymol 347: 40-9.

Copeland, P. R., J. E. Fletcher, et al. (2000). "A novel RNA binding protein, SBP2, is required for the translation of mammalian selenoprotein mRNAs." EMBO J 19(2): 306-14.

Copeland, P. R., V. A. Stepanik, et al. (2001). "Insight into mammalian selenocysteine insertion: domain structure and ribosome binding properties of Sec insertion sequence binding protein 2." Mol Cell Biol 21(5): 1491-8.

de Jesus, L. A., P. R. Hoffmann, et al. (2006). "Nuclear assembly of UGA decoding complexes on selenoprotein mRNAs: a mechanism for eluding nonsense-mediated decay?" Mol Cell Biol 26(5): 1795-805.

de Kinderen, R. J., D. A. Lambrechts, et al. (2011). "Research into the (Cost-) effectiveness of the ketogenic diet among children and adolescents with intractable epilepsy: design of a randomized controlled trial." BMC Neurol 11(1): 10.

de Lanerolle, N. C. and T. S. Lee (2005). "New facets of the neuropathology and molecular profile of human temporal lobe epilepsy." Epilepsy Behav 7(2): 190-203.

Dreher, I., C. Schmutzler, et al. (1997). "Expression of selenoproteins in various rat and human tissues and cell lines." Journal of Trace Elements in Medicine and Biology 11(2): 83-91.

Dumitrescu, A. M., X. H. Liao, et al. (2005). "Mutations in SECISBP2 result in abnormal thyroid hormone metabolism." Nat Genet 37(11): 1247-52.

El-Sayed, W. M., T. Aboul-Fadl, et al. (2006). "Effect of selenium-containing compounds on hepatic chemoprotective enzymes in mice." Toxicology 220(2-3): 179-88.

Fabre, R. and R. Truhaut (1956). "Toxicological study of selenium." Rev Pathol Gen Physiol Clin 56(675): 323-39.

Farrell, K., E. Wirrell, et al. (2006). "The definition and prediction of intractable epilepsy in children." Adv Neurol 97: 435-42.

Fels, I. G. and V. H. Cheldelin (1949). "Selenate inhibition studies; the role of sulfate in selenate toxicity in yeast." Arch Biochem 22(3): 402-5.

Feng, Y. K. (1978). "Some statistics on 2,810 cases of epilepsy." Chin Med J (Engl) 4(6): 449-56.

Finkel, T. (2000). "Redox-dependent signal transduction." FEBS Lett 476(1-2): 52-4.

Fisher, R. S., W. van Emde Boas, et al. (2005). "Epileptic seizures and epilepsy: definitions proposed by the International League Against Epilepsy (ILAE) and the International Bureau for Epilepsy (IBE)." Epilepsia 46(4): 470-2.

Fletcher, J. E., P. R. Copeland, et al. (2001). "The selenocysteine incorporation machinery: interactions between the SECIS RNA and the SECIS-binding protein SBP2." RNA 7(10): 1442-53.

Flohe, L., W. A. Gunzler, et al. (1973). "Glutathione peroxidase: a selenoenzyme." FEBS Lett 32(1): 132-4.

Flohe, L., H. J. Hecht, et al. (1999). "Glutathione and trypanothione in parasitic hydroperoxide metabolism." Free Radic Biol Med 27(9-10): 966-84.

Fountain, N. B., P. C. Van Ness, et al. (2011). "Quality improvement in neurology: AAN epilepsy quality measures: Report of the Quality Measurement and Reporting Subcommittee of the American Academy of Neurology." Neurology 76(1): 94-9.

Garjani, A., F. Fathiazad, et al. (2009). "The effect of total extract of Securigera securidaca L. seeds on serum lipid profiles, antioxidant status, and vascular function in hypercholesterolemic rats." J Ethnopharmacol 126(3): 525-32. 
Go, C. and O. C. Snead, 3rd (2008). "Pharmacologically intractable epilepsy in children: diagnosis and preoperative evaluation." Neurosurg Focus 25(3): E2.

Gomez-Alonso, J. and B. G. Giraldez (2007). "Epilepsy: a new definition for an old disease." Rev Neurol 45(2): 126-7.

Greenberg, D. A. and R. Subaran (2011). "Blinders, phenotype, and fashionable genetic analysis: a critical examination of the current state of epilepsy genetic studies." Epilepsia 52(1): 1-9.

Guanqing, H. (1979). "On the etiology of Keshan disease: two hypotheses." Chin Med J (Engl) 92(6): 416-22.

Haut, S. R., R. B. Lipton, et al. (2005). "Identifying seizure clusters in patients with epilepsy." Neurology 65(8): 1313-5.

Hawkes, W. C. and Z. Alkan (2010). "Regulation of redox signaling by selenoproteins." Biol Trace Elem Res 134(3): 235-51.

Hazebrouck, S., L. Camoin, et al. (2000). "Substituting selenocysteine for catalytic cysteine 41 enhances enzymatic activity of plant phospholipid hydroperoxide glutathione peroxidase expressed in Escherichia coli." Journal of Biological Chemistry 275(37): 28715-21.

Hill, K. E., J. Zhou, et al. (2007). "The selenium-rich C-terminal domain of mouse selenoprotein $\mathrm{P}$ is necessary for the supply of selenium to brain and testis but not for the maintenance of whole body selenium." Journal of Biological Chemistry 282(15): 10972-80.

Hill, K. E., J. Zhou, et al. (2003). "Deletion of selenoprotein P alters distribution of selenium in the mouse." Journal of Biological Chemistry 278(16): 13640-6.

Hill, K. E., J. Zhou, et al. (2004). "Neurological dysfunction occurs in mice with targeted deletion of the selenoprotein P gene." Journal of Nutrition 134(1): 157-61.

Hoffmann, P. R., S. C. Hoge, et al. (2007). "The selenoproteome exhibits widely varying, tissue-specific dependence on selenoprotein P for selenium supply." Nucleic Acids Research 35(12): 3963-73.

Hoffmann, P. R., S. C. Hoge, et al. (2007). "The selenoproteome exhibits widely varying, tissue-specific dependence on selenoprotein P for selenium supply." Nucleic Acids Research 35(12): 3963-3973.

Holmgren, A. and M. Bjornstedt (1995). "Thioredoxin and thioredoxin reductase." Methods Enzymol 252: 199-208.

Hondal, R. J. and E. L. Ruggles (2011). "Differing views of the role of selenium in thioredoxin reductase." Amino Acids 41(1):73-89.

Howard, M. T., M. W. Moyle, et al. (2007). "A recoding element that stimulates decoding of UGA codons by Sec tRNA[Ser]Sec." RNA 13(6): 912-20.

Howse, D. C. and T. E. Duffy (1975). "Control of the redox state of the pyridine nucleotides in the rat cerebral cortex. Effect of electroshock-induced seizures." J Neurochem 24(5): 935-40.

Hwang, D. Y., J. S. Cho, et al. (2005). "Differentially expressed genes in transgenic mice carrying human mutant presenilin-2 (N141I): correlation of selenoprotein M with Alzheimer's disease." Neurochem Res 30(8): 1009-19.

Imam, S. Z., G. D. Newport, et al. (1999). "Selenium, an antioxidant, protects against methamphetamine-induced dopaminergic neurotoxicity." Brain Research 818(2): 575-578. 
Ito, M., N. Echizenya, et al. (2009). "A case series of epilepsy-derived memory impairment resembling Alzheimer disease." Alzheimer Dis Assoc Disord 23(4): 406-9.

Jackson, M. I. and G. F. Combs, Jr. (2008). "Selenium and anticarcinogenesis: underlying mechanisms." Curr Opin Clin Nutr Metab Care 11(6): 718-26.

Jaseja, H. (2009). "Definition of epilepsy: significance of its revision on clinical neurophysiological basis to improve prognosis and quality of life of patients with epilepsy." Med Hypotheses 72(6): 756-7.

Kakturskii, L. V., L. S. Strochkova, et al. (1990). "Hyposelenoses." Arkh Patol 52(12): 3-8.

Klug, H. L., R. D. Harshfield, et al. (1952). "Methionine and selenium toxicity." Journal of Nutrition 48(4): 409-20.

Korotkov, K. V., S. V. Novoselov, et al. (2002). "Mammalian selenoprotein in which selenocysteine (Sec) incorporation is supported by a new form of Sec insertion sequence element." Mol Cell Biol 22(5): 1402-11.

Kortt, A. A., J. B. Caldwell, et al. (1991). "Amino acid and cDNA sequences of a methioninerich $2 S$ protein from sunflower seed (Helianthus annuus L.)." Eur J Biochem 195(2): 329-34.

Kossoff, E. H. and J. R. McGrogan (2005). "Worldwide use of the ketogenic diet." Epilepsia 46(2): 280-9.

Kryukov, G. V., S. Castellano, et al. (2003). "Characterization of mammalian selenoproteomes." Science 300(5624): 1439-43.

Kryukov, G. V. and V. N. Gladyshev (2002). "Mammalian selenoprotein gene signature: identification and functional analysis of selenoprotein genes using bioinformatics methods." Methods Enzymol 347: 84-100.

Kutluhan, S., M. Naziroglu, et al. (2009). "Effects of selenium and topiramate on lipid peroxidation and antioxidant vitamin levels in blood of pentylentetrazol-induced epileptic rats." Biol Trace Elem Res 129(1-3): 181-9.

Kwan, P. and M. J. Brodie (2000). "Early identification of refractory epilepsy." New England Journal of Medicine 342(5): 314-319.

Ladiges, W., H. Van Remmen, et al. (2009). "Lifespan extension in genetically modified mice." Aging Cell 8(4): 346-52.

Lamarche, F., N. Signorini-Allibe, et al. (2004). "Influence of vitamin E, sodium selenite, and astrocyte-conditioned medium on neuronal survival after chronic exposure to ethanol." Alcohol 33(2): 127-38.

Landre, E. (2004). "[Vagus nerve stimulation and refractory partial epilepsies]." Rev Neurol (Paris) 160 Spec No 1: 5S280-7.

Larner, A. J. (2010). "Epileptic seizures in AD patients." Neuromolecular Med 12(1): 71-7.

Lee, B. J., P. J. Worland, et al. (1989). "Identification of a selenocysteyl-tRNA(Ser) in mammalian cells that recognizes the nonsense codon, UGA." Journal of Biological Chemistry 264(17): 9724-7.

Lee, S. R., S. Bar-Noy, et al. (2000). "Mammalian thioredoxin reductase: oxidation of the Cterminal cysteine/selenocysteine active site forms a thioselenide, and replacement of selenium with sulfur markedly reduces catalytic activity." Proc Natl Acad Sci U S A 97(6): 2521-6.

Lei, X. G., W. H. Cheng, et al. (2007). "Metabolic regulation and function of glutathione peroxidase-1." Annu Rev Nutr 27: 41-61. 
Leinfelder, W., T. C. Stadtman, et al. (1989). "Occurrence in vivo of selenocysteyltRNA(SERUCA) in Escherichia coli. Effect of sel mutations." Journal of Biological Chemistry 264(17): 9720-3.

Lerman, P., S. Sagie, et al. (2011). "Why can seizures remain intractable? Clinical vignettes from the life experience of a pediatric epileptologist." J Child Neurol 26(1): 121-5.

Lescure, A., C. Allmang, et al. (2002). "cDNA cloning, expression pattern and RNA binding analysis of human selenocysteine insertion sequence (SECIS) binding protein 2." Gene 291(1-2): 279-85.

Lescure, A., D. Fagegaltier, et al. (2002). "Protein factors mediating selenoprotein synthesis." Curr Protein Pept Sci 3(1): 143-51.

Low, S. C., E. Grundner-Culemann, et al. (2000). "SECIS-SBP2 interactions dictate selenocysteine incorporation efficiency and selenoprotein hierarchy." EMBO J 19(24): 6882-90.

Lu, J. and A. Holmgren (2009). "Selenoproteins." Journal of Biological Chemistry 284(2): 7237.

Maganti, R., P. Gerber, et al. (2008). "Nonconvulsive status epilepticus." Epilepsy Behav 12(4): 572-86.

Maillard, L., J. P. Vignal, et al. (2009). "Risk of epilepsy after a first epileptic seizure in adults: Can we predict the future?." Rev Neurol (Paris) 165(10): 782-8.

Mc, C. K. and O. W. Portman (1952). "Toxicity of dimethyl selenide in the rat and mouse." Proc Soc Exp Biol Med 79(2): 230-1.

Mehla, J., K. H. Reeta, et al. (2010). "Protective effect of curcumin against seizures and cognitive impairment in a pentylenetetrazole-kindled epileptic rat model." Life Sci 87(19-22): 596-603.

Meinardi, H., R. A. Scott, et al. (2001). "The treatment gap in epilepsy: the current situation and ways forward." Epilepsia 42(1): 136-49.

Mertz, W. and K. Schwarz (1958). "Reversal of respiratory decline in necrotic liver degeneration by intraportal antioxidants." Proc Soc Exp Biol Med 98(4): 808-12.

Micke, O., L. Schomburg, et al. (2009). "Selenium in oncology: from chemistry to clinics." Molecules 14(10): 3975-88.

Militao, G. C., P. M. Ferreira, et al. (2010). "Effects of lipoic acid on oxidative stress in rat striatum after pilocarpine-induced seizures." Neurochem Int 56(1): 16-20.

Mix, H., A. V. Lobanov, et al. (2007). "SECIS elements in the coding regions of selenoprotein transcripts are functional in higher eukaryotes." Nucleic Acids Research 35(2): 41423.

Nakayama, A., K. E. Hill, et al. (2007). "All regions of mouse brain are dependent on selenoprotein P for maintenance of selenium." Journal of Nutrition 137(3): 690-693.

Nakken, K. O. and E. Tauboll (2009). "Drug-resistant epilepsy." Tidsskr Nor Laegeforen 129(19): 1986-9.

Naziroglu, M. (2009). "Role of Selenium on Calcium Signaling and Oxidative Stress-induced Molecular Pathways in Epilepsy." Neurochem Res 34(12):2181-91.

Noebels, J. (2011). "A perfect storm: Converging paths of epilepsy and Alzheimer's dementia intersect in the hippocampal formation." Epilepsia 52 Suppl (1): 39-46.

Nohl, H., D. Hegner, et al. (1979). "Responses of mitochondrial superoxide dismutase, catalase and glutathione peroxidase activities to aging." Mech Ageing Dev 11(3): $145-51$. 
Novoselov, S. V., G. V. Kryukov, et al. (2007). "Selenoprotein H is a nucleolar thioredoxinlike protein with a unique expression pattern." Journal of Biological Chemistry 282(16): 11960-8.

Pages, N., P. Maurois, et al. (2010). "Activities of alpha-asarone in various animal seizure models and in biochemical assays might be essentially accounted for by antioxidant properties." Neurosci Res 68(4): 337-44.

Palop, J. J. and L. Mucke (2009). "Epilepsy and cognitive impairments in Alzheimer disease." Arch Neurol 66(4): 435-40.

Panee, J., Z. R. Stoytcheva, et al. (2007). "Selenoprotein H is redox-sensing high mobility group family DNA-binding protein that up-regulates genes involved in glutathione synthesis and phase II detoxification." Journal of Biological Chemistry 282(33): 23759-23765.

Papp, L. V., J. Lu, et al. (2007). "From selenium to selenoproteins: synthesis, identity, and their role in human health." Antioxid Redox Signal 9(7): 775-806.

Poduri, A. and D. Lowenstein (2011). "Epilepsy genetics-past, present, and future." Curr Opin Genet Dev 21(3):325-32.

Prunetti, P. and E. Perucca (2011). "New and forthcoming anti-epileptic drugs." Curr Opin Neurol 24(2): 159-64.

Puzanowska-Tarasiewicz, H., L. Kuzmicka, et al. (2009). "Biological function of some elements and their compounds. II. Selenium, selenate, selenium organic compounds." Pol Merkur Lekarski 27(159): 249-52.

Ramaekers, V. T., N. Calomme, et al. (1994). "Selenium Deficiency Triggering Intractable Seizures." Neuropediatrics 25(4): 217-223.

Ran, Q., H. Liang, et al. (2007). "Reduction in glutathione peroxidase 4 increases life span through increased sensitivity to apoptosis." J Gerontol A Biol Sci Med Sci 62(9): 93242.

Rao, S. C., G. Dove, et al. (2009). "Recurrent seizures in patients with dementia: frequency, seizure types, and treatment outcome." Epilepsy Behav 14(1): 118-20.

Rees, M. I. (2010). "The genetics of epilepsy--the past, the present and future." Seizure 19(10): 680-3.

Reeves, M. A., F. P. Bellinger, et al. (2010). "The neuroprotective functions of selenoprotein $\mathrm{M}$ and its role in cytosolic calcium regulation." Antioxid Redox Signal 12(7): 809-18.

Renko, K., P. J. Hofmann, et al. (2009). "Down-regulation of the hepatic selenoprotein biosynthesis machinery impairs selenium metabolism during the acute phase response in mice." Faseb Journal 23(6): 1758-1765.

Rotruck, J. T., A. L. Pope, et al. (1973). "Selenium: biochemical role as a component of glutathione peroxidase." Science 179(73): 588-90.

Rubin, J. J. and L. J. Willmore (1980). "Prevention of Iron-Induced Epileptiform Discharges in Rats by Treatment with Anti-Peroxidants." Experimental Neurology 67(3): 472-480.

Saito, Y., T. Hayashi, et al. (1999). "Selenoprotein P in human plasma as an extracellular phospholipid hydroperoxide glutathione peroxidase. Isolation and enzymatic characterization of human selenoprotein p." Journal of Biological Chemistry 274(5): 2866-71.

Sander, J. W. A. S. (1993). "Some Aspects of Prognosis in the Epilepsies - a Review." Epilepsia 34(6): 1007-1016. 
Santamaria, A., B. Vazquez-Roman, et al. (2005). "Selenium reduces the proapoptotic signaling associated to NF-kappaB pathway and stimulates glutathione peroxidase activity during excitotoxic damage produced by quinolinate in rat corpus striatum." Synapse 58(4): 258-66.

Savaskan, N. E., A. U. Brauer, et al. (2003). "Selenium deficiency increases susceptibility to glutamate-induced excitotoxicity." Faseb Journal 17(1): 112-4.

Scarmeas, N., L. S. Honig, et al. (2009). "Seizures in Alzheimer disease: who, when, and how common?" Arch Neurol 66(8): 992-7.

Schomburg, L., U. Schweizer, et al. (2003). "Gene disruption discloses role of selenoprotein P in selenium delivery to target tissues." Biochem J 370(Pt 2): 397-402.

Schwarz, K., L. A. Porter, et al. (1972). "Some regularities in the structure-function relationship of organoselenium compounds effective against dietary liver necrosis." Ann N Y Acad Sci 19(2): 200-14.

Schwarz, K., J. A. Stesney, et al. (1959). "Relation between selenium traces in L-cystine and protection against dietary liver necrosis." Metabolism 8(1): 88-90.

Schweizer, U., A. U. Brauer, et al. (2004). "Selenium and brain function: a poorly recognized liaison." Brain Res Brain Res Rev 45(3): 164-78.

Sharma, V., B. Nehru, et al. (2010). "Antioxidant potential of curcumin against oxidative insult induced by pentylenetetrazol in epileptic rats." Methods Find Exp Clin Pharmacol 32(4): 227-32.

Sisodiya, S. M. and H. C. Mefford (2011). "Genetic contribution to common epilepsies." Curr Opin Neurol 24(2): 140-5.

Small-Howard, A. L. and M. J. Berry (2005). "Unique features of selenocysteine incorporation function within the context of general eukaryotic translational processes." Biochem Soc Trans 33(Pt 6): 1493-7.

Sozmen, V., S. Baybas, et al. (2011). "Frequency of epilepsies in family members of patients with different epileptic syndromes." Eur Neurol 65(1): 4-9.

Squires, J. E. and M. J. Berry (2008). "Eukaryotic selenoprotein synthesis: mechanistic insight incorporating new factors and new functions for old factors." IUBMB Life 60(4): 232-5.

Stadtman, T. C., J. N. Davis, et al. (1989). "Biochemical and genetic analysis of Salmonella typhimurium and Escherichia coli mutants defective in specific incorporation of selenium into formate dehydrogenase and tRNAs." Biofactors 2(1): 35-44.

Stefani, A., F. Spadoni, et al. (1997). "Voltage-activated calcium channels: targets of antiepileptic drug therapy?" Epilepsia 38(9): 959-65.

Steinert, P., D. Bachner, et al. (1998). "Analysis of the mouse selenoprotein P gene." Biological Chemistry 379(6): 683-691.

Steinert, T., H. Baier, et al. (2011). "Epileptic Seizures During Treatment with Antidepressants and Neuroleptics." Fortschr Neurol Psychiatr 79(3): 138-143.

Strzelczyk, A., M. Cenusa, et al. (2011). "Management and long-term outcome in patients presenting with ictal asystole or bradycardia." Epilepsia 52(6):1160-7.

Sun, Y., J. A. Butler, et al. (2001). "Glutathione peroxidase activity and selenoprotein W levels in different brain regions of selenium-depleted rats." Journal of Nutritional Biochemistry 12(2): 88-94.

Suzuki, Y. (2011). "Molecular basis of neurogenetic diseases." Brain Dev. doi:10.1016/ j.physletb.2003.10.071 
Takebe, G., J. Yarimizu, et al. (2002). "A comparative study on the hydroperoxide and thiol specificity of the glutathione peroxidase family and selenoprotein P." Journal of Biological Chemistry 277(43): 41254-8.

Tamura, T. and T. C. Stadtman (2002). "Mammalian thioredoxin reductases." Methods Enzymol 347: 297-306.

Tome Ada, R., P. M. Ferreira, et al. (2010). "Inhibitory action of antioxidants (ascorbic acid or alpha-tocopherol) on seizures and brain damage induced by pilocarpine in rats." Arq Neuropsiquiatr 68(3): 355-61.

Tsai, J. J. (2005). "Mortality of epilepsy from national vital statistics and University epilepsy clinic in Taiwan." Epilepsia 46 Suppl 1(1): 8-10.

Tujebajeva, R. M., P. R. Copeland, et al. (2000). "Decoding apparatus for eukaryotic selenocysteine insertion." EMBO Rep 1(2): 158-63.

Urbach, H. (2005). "Imaging of the epilepsies." Eur Radiol 15(3): 494-500.

Vendeland, S. C., M. A. Beilstein, et al. (1993). "Purification and properties of selenoprotein W from rat muscle." Journal of Biological Chemistry 268(23): 17103-7.

Verrotti, A., G. Loiacono, et al. (2011). "Pharmacotherapy for children and adolescents with epilepsy." Expert Opin Pharmacother 12(2): 175-94.

Vezzani, A., J. French, et al. (2011). "The role of inflammation in epilepsy." Nat Rev Neurol $7(1): 31-40$.

Wang, G. S., D. Q. Geng, et al. (2010). "Protective effect of $\mathrm{Na}_{2} \mathrm{SeO}_{3}$ against cerebral ischemia-reperfusion injury to the hippocampal neurons in rats." Nan Fang Yi Ke Da Xue Xue Bao 30(10): 2336-9.

Weber, G. F., P. Maertens, et al. (1991). "Glutathione peroxidase deficiency and childhood seizures." Lancet 337(8755): 1443-4.

Westmark, C. J., P. R. Westmark, et al. (2010). "Alzheimer's disease and Down syndrome rodent models exhibit audiogenic seizures." J Alzheimers Dis 20(4): 1009-13.

Whanger, P. D. (2009). "Selenoprotein expression and function-selenoprotein W." Biochim Biophys Acta 1790(11): 1448-52.

Wilke, C., G. Worrell, et al. (2011). "Graph analysis of epileptogenic networks in human partial epilepsy." Epilepsia 52(1): 84-93.

Willmore, L. J. and J. J. Rubin (1981). "Antiperoxidant pretreatment and iron-induced epileptiform discharges in the rat: EEG and histopathologic studies." Neurology 31(1): 63-9.

Wirth, E. K., M. Conrad, et al. (2010). "Neuronal selenoprotein expression is required for interneuron development and prevents seizures and neurodegeneration." FASEB J 24(3): 844-52.

Wu, M., M. M. Kang, et al. (2010). "Selenium compounds activate early barriers of tumorigenesis." Journal of Biological Chemistry 285(16): 12055-62.

Xiong, S., W. R. Markesbery, et al. (2007). "Seleno-L-methionine protects against betaamyloid and iron/hydrogen peroxide-mediated neuron death." Antioxid Redox Signal 9(4): 457-67.

Xu, X. M., B. A. Carlson, et al. (2007). "New developments in selenium biochemistry: selenocysteine biosynthesis in eukaryotes and archaea." Biol Trace Elem Res 119(3): $234-41$. 
Yang, G. Q. and Y. M. Xia (1995). "Studies on human dietary requirements and safe range of dietary intakes of selenium in China and their application in the prevention of related endemic diseases." Biomed Environ Sci 8(3): 187-201.

Yang, X. E., W. R. Chen, et al. (2007). "Improving human micronutrient nutrition through biofortification in the soil-plant system: China as a case study." Environ Geochem Health 29(5): 413-28.

Yasuda, S., H. Sugiura, et al. (2011). "p38 Map Kinase Inhibitors as Potential Therapeutic Drugs for Neural Diseases." Cent Nerv Syst Agents Med Chem 11(1): 45-59.

Yuzbasioglu, A., H. Karatas, et al. (2009). "Changes in the expression of selenoproteins in mesial temporal lobe epilepsy patients." Cell Mol Neurobiol 29(8): 1223-31.

Zafar, K. S., A. Siddiqui, et al. (2003). "Dose-dependent protective effect of selenium in rat model of Parkinson's disease: neurobehavioral and neurochemical evidences." Journal of Neurochemistry 84(3): 438-446.

Zhang, S., C. Rocourt, et al. (2010). "Selenoproteins and the aging brain." Mech Ageing Dev 131(4): 253-60.

Zhang, Y., Y. Zhou, et al. (2008). "Comparative analysis of selenocysteine machinery and selenoproteome gene expression in mouse brain identifies neurons as key functional sites of selenium in mammals." Journal of Biological Chemistry 283(4): 2427-2438. 


\section{Part 2}

Molecular Genetics of Epilepsy 



\title{
The Molecular Genetics of the Benign Epilepsies of Infancy
}

\author{
Sarah E. Heron ${ }^{1}$ and John C. Mulley ${ }^{2,3}$ \\ ${ }^{1}$ University of South Australia \\ 2SA Pathology at Women's and Children's Hospital, \\ ${ }^{3}$ University of Adelaide \\ Australia
}

\section{Introduction}

Three benign epilepsy syndromes with autosomal dominant inheritance are recognised in infancy. These are Benign Familial Neonatal Epilepsy (BFNE), Benign Familial NeonatalInfantile Epilepsy (BFNIE) and Benign Familial Infantile Epilepsy (BFIE). These disorders were previously known as Benign Familial Neonatal Seizures, Benign Familial NeonatalInfantile Seizures and Benign Familial Infantile Seizures, respectively (Berg et al., 2010). They were recently renamed by the International League Against Epilepsy Classification Committee. The three disorders differ in their average ages of onset but age of onset distributions overlap between the syndromes. Ages of onset vary within syndromes and within families affected with any of the syndromes.

BFNE patients have lateralised motor seizures with a mean age of onset of around 3 days of age and a mean age of seizure offset of 3 months of age. Subsequent neurological development usually proceeds normally. However, about $15 \%$ of patients have later seizures or epilepsy and occasional cases deteriorate into severe epileptic encephalopathies with intellectual disability (Dedek et al., 2003; Borgatti et al., 2004; Steinlein et al., 2007). The benign form of the disorder was originally described in an Austrian family by Rett and Teubel (1964). Since then numerous BFNE families have been described, many with potassium channel subunit mutations, to provide a well established range of presentations.

BFIE was originally described by Watanabe and colleagues in 1987, and is sometimes referred to as Watanabe syndrome. Watanabe observed that a subgroup of infants with generalised motor seizures had subsequent normal development and became seizure-free by 3 years of age. Similar families were subsequently described by Vigevano and colleagues (1992). Onset of seizures in BFIE occurs between 4 and 6 months of age with seizure offset generally occurring by 12 months of age. Seizures are generally not seen in later life. However, some cases develop paroxysmal dyskinesia or paroxysmal kinesigenic choreoathetosis in adolescence. This is known as infantile convulsions and choreoathetosis (ICCA) syndrome.

BFNIE patients have similar seizures to those seen in BFNE and BFIE, with the average age of onset intermediate between the other two. Although BFNIE differs from these disorders in the average age of seizure onset, the age of onset distribution which can range within a single family from 3 days up to 13 months of age (Herlenius et al., 2007), significantly 
overlaps with BFNE and BFIE. Seizure onset in BFNIE most commonly occurs at between 2 and 4 months of age. Clinically the condition was first described by Kaplan and Lacey (1983) and molecularly delineated two decades later (Heron et al., 2002).

The aim of this chapter is to describe the current state of knowledge of the molecular genetics underlying these disorders and the significance that this has for patient care.

\section{The molecular genetics of benign familial neonatal epilepsy}

Benign familial neonatal epilepsy is most commonly caused by mutations in two related voltage-gated potassium channel subunit genes, KCNQ2 and KCNQ3. Together, these form the pore of the neuronal M-channel, which plays an important role in neuronal inhibition by acting as a "brake" on repetitive action potential discharges (Delmas \& Brown, 2005). Mutations in KCNQ2 and KCNQ3 were originally described in 1998 (Biervert et al., 1998; Charlier et al., 1998; Singh et al., 1998). There are now more than 80 known mutations in KCNQ2; but only four described for KCNQ3. There may be other, rare, families with mutations in other genes.

\subsection{BFNE caused by mutations in the potassium channel subunit KCNQ2}

KCNQ2 (OMIM 602235) encodes one of the pore-forming subunits of the M-channel. The Mchannel is a heteromultimer consisting of two KCNQ2 and two KCNQ3 proteins, as well as accessory subunits. The gene is located close to the q-arm telomere of chromosome 20, at 20q13.3. It consists of 17 exons embedded within $87 \mathrm{~kb}$ of genomic DNA. The gene codes for an 872 amino acid protein consisting of three domains: a short N-terminal domain; a transmembrane domain; and a large C-terminal domain, which contains regions that interact with other channel subunits. The gene has five splice variants, which produce proteins that differ in the C-terminal domains. The transmembrane domain consists of six transmembrane segments (S1-S6) and the pore-loop between segments S5 and S6, which forms the lining of the ion pore. The $\mathrm{S} 4$ domain is the voltage sensor and contains a number of positively charged arginine or lysine residues, which are required for normal channel function. The S4 segments move within the membrane in response to a change in voltage, opening the channel.

BFNE was originally mapped to the KCNQ2 locus in 1989 (Leppert et al.) by linkage analysis of a large, four-generation pedigree. Further families were reported that confirmed the chromosome 20q localisation (Ryan et al., 1991; Malafosse et al., 1992). KCNQ2 was identified as the BFNE gene at this locus when a submicroscopic deletion containing KCNQ2 was detected in a single BFNE family. Screening of additional BFNE families revealed missense, frameshift and splice-site mutations in KCNQ2 (Singh et al., 1998). A frameshift mutation was simultaneously identified in KCNQ2 when it was screened as a candidate gene in another family mapped to the 20q locus (Biervert et al., 1998).

In total, 85 mutations in $K C N Q 2$ have been described in the scientific literature (Heron et al., 2007; Soldovieri et al., 2007; Wuttke et al., 2007; Yalçin et al., 2007; Neubauer et al. 2008; Sadewa et al., 2008; Goldberg-Stern et al., 2009; Ishii et al., 2009; Kurahashi et al. 2009; Lee et al., 2009; Miceli et al., 2009; Volkers et al. 2009; Yum et al., 2010). These include missense, nonsense, splice site and frameshift mutations as well as deletions or duplications affecting multiple exons. The latter type of mutation accounts for over $10 \%$ of the published $K C N Q 2$ mutations and for around $20 \%$ of the KCNQ2 mutations in one BFNE cohort 
comprehensively analysed for all classes of mutation (Heron et al., 2007). Several deletions have been described which also affect the genes surrounding KCNQ2 (Kurahashi et al., 2009). Patients carrying these deletions do not exhibit different phenotypes to BFNE patients with KCNQ2 missense mutations, indicating that the loss of one copy of these contiguous genes is no more pathogenic than the loss of one copy of KCNQ2 alone. The deletions and duplications affecting KCNQ2 are shown in Figure 1.

Approximately one-third of the mutations in $K C N Q 2$ are missense mutations. Unlike the truncating mutations, which are spread throughout the gene, most of the missense changes occur in regions of functional importance. A cluster of missense mutations is seen in the transmembrane domain, with mutations particularly frequent in the S4 segment and the pore-loop. Functional studies of some of these mutations showed that they reduce channel activity and thus reduce neuronal inhibition (Soldovieri et al., 2007). Clusters of missense mutations are also seen in two regions of the C-terminal domain involved in binding to calmodulin. This interaction is known to be required for optimal channel function and mutations affecting it can disrupt the interaction and thus the normal function of the Mchannel (Wen and Levitan, 2002; Yus-Najera et al., 2002; Richards et al., 2004).

The published mutations in KCNQ2 are summarised in Table 1. More than half of the mutations in KCNQ2 are either truncating mutations or large deletions or duplications that disrupt the structure of the gene. Both of these types of mutation extinguish protein expression from the mutated allele. Haploinsufficiency is the most significant mutational mechanism affecting KCNQ2 that manifests as BFNE.

\begin{tabular}{|ll|}
\hline Mutation Type & Number Reported \\
\hline Start Codon & 2 \\
\hline Missense & 28 \\
\hline Nonsense & 9 \\
\hline Frameshift & 21 \\
In-frame deletion & 3 \\
\hline Splice site & 11 \\
\hline Large deletion or duplication & 10 \\
\hline Other & 1 \\
Total & $\mathbf{8 5}$ \\
\hline
\end{tabular}

Table 1. Summary of mutations reported in KCNQ2

\subsection{BFNE caused by mutations in the potassium channel subunit KCNQ3}

KCNQ3 (OMIM 602232) encodes the second pore-forming subunit of the M-channel. The $K C N Q 3$ gene is located on the long arm of chromosome 8, at 8q24, and consists of 15 exons spread across $360 \mathrm{~kb}$ of genomic DNA. The 849 amino acid KCNQ3 protein has a structure very similar to that of the KCNQ2 protein described above. The two proteins have highly homologous sequences in the transmembrane domains, but the sequences of the $\mathrm{N}$ - and Cterminal domains differ markedly (GenBank accession numbers NP_742105 and NP_004510).

Following the linkage of BFNE to the KCNQ2 locus at 20q13.3, families were described that did not link to the region, indicating the presence of a second locus for the disorder. Some of these families were found to have evidence of linkage to a locus on chromosome 8q (Lewis et al., 1993; Steinlein et al., 1995) where the KCNQ3 gene, with similarities to the KCNQ2 
gene, was known to map. A mutation in $K C N Q 3$ was described at the same time as the first mutations in KCNQ2 (Charlier et al., 1998).

Only four mutations in KCNQ3 associated with BFNE have been reported in the literature. All of these affect amino acid residues located in or near the pore-loop (Charlier et al., 1998; Hirose et al., 2000; Singh et al., 2003; Li et al., 2008a). These mutations have not been functionally investigated, so their precise effect on channel function is unknown. The transient occurrence of seizures in BFNE may be due to changes in the expression of KCNQ2 and KCNQ3 that occur during development (Weber et al., 2006; Geiger et al., 2006; Kanaumi et al., 2008).

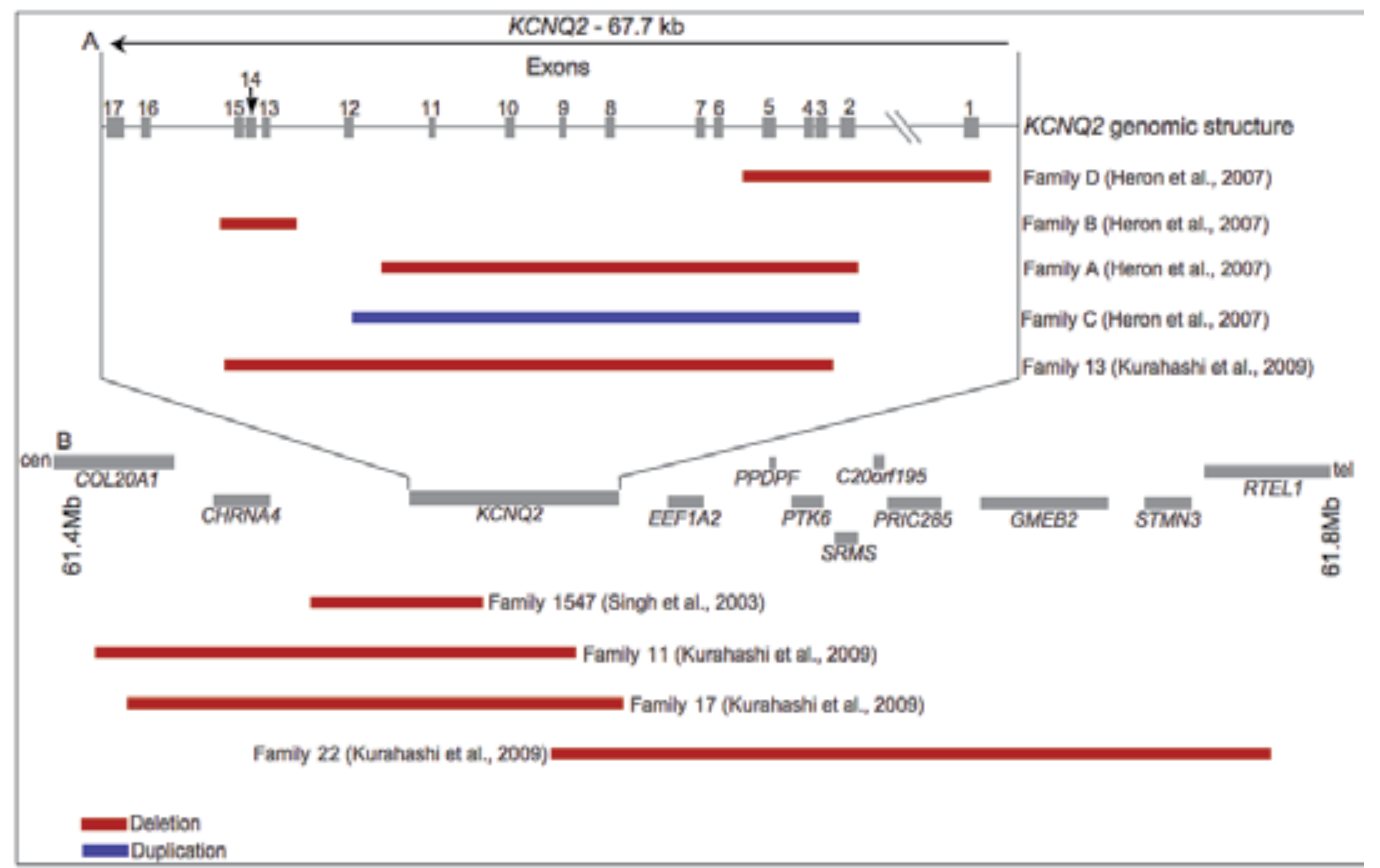

Fig. 1. Nine microchromosomal rearrangements described in KCNQ2. A: Intragenic deletions (red) and duplication (blue). B: Deletions extending beyond KCNQ2. A tenth deletion has been reported (Steinlein et al., 2007) but its breakpoints were not described.

\subsection{BFNE with other causes}

Occasional cases of BFNE have been reported with causes other than mutations in KCNQ2 or KCNQ3. A small family with a pericentromeric inversion on chromosome 5 was reported in 2002 by Concolino and colleagues. The breakpoints for this inversion were mapped to 5 p15.1 and 5q11.2. This inversion is presumed to disrupt a gene at one of these breakpoints, but this gene has not been identified. However, it is possible that further BFNE families have missense or other mutations affecting this gene on chromosome 5 . It is also possible that the inversion disrupts a regulatory sequence for a gene located at some distance from the breakpoint.

Mutations have also been reported that cause BFNE with co-morbidities (Dedek et al., 2001; Dedek et al., 2003; Borgatti et al. 2004; Schmitt et al., 2005; Stenlein et al., 2007). Recently a 
family with familial neonatal seizures and intellectual disability caused by a microduplication at chromosome 2q24.3 has been reported (Heron et al., 2010). The four affected members of this family all had neonatal seizures and some degree of intellectual disability ranging from borderline intelligence to an IQ score less than 40. A second patient with a similar microduplication has been described with seizures starting at 2-3 weeks of age and developmental delay (Raymond et al., 2011). This suggests that microduplications at chromosome $2 \mathrm{q} 24.3$, which contains a cluster of five voltage-gated sodium channel genes, could be a recurrent cause of neonatal seizures accompanied by intellectual disability. Such mutations can be easily detected and characterised using comparative genome hybridisation (CGH), therefore patients with neonatal seizures and intellectual disability should be tested for microduplications using this method. The pathogenic mechanism of these microduplications is hypothesised to be an increase in the expression of the duplicated sodium channel genes, in contrast to the haploinsufficiency cause by truncating or deletion mutations or the changes in function caused by missense mutations in KCNQ2. The two duplications described so far have slightly different breakpoints (Raymond et al., 2011), but are probably both the result of non-homologous recombination between the five sodium channel genes in the region.

These reports suggest that not all cases of BFNE are caused by KCNQ2 and KCNQ3 mutations. However, these exceptions are not common as mutations in these two genes account for around $90 \%$ of cases of BFNE.

\section{The molecular genetics of benign familial neonatal-infantile epilepsy}

Patients with benign familial neonatal-infantile epilepsy (BFNIE) have similar seizures to those seen in BFNE and BFIE. BFNIE differs from these disorders in the age of onset, which can range within a single family from 3 days up to 13 months of age (Herlenius et al., 2007), although seizure onset in BFNIE most commonly occurs at between 2 and 4 months. BFNIE was first described as a distinct clinical entity by Kaplan and Lacey in 1983. They described an American family with 12 affected individuals who had seizures with onset ranging from 3 days to $3 \frac{1}{2}$ months. All these individuals had subsequent normal development. Another family was subsequently described with seizure onset also occurring between 3 days and $3^{1 / 2}$ months of age (Lewis et al., 1996). Linkage to KCNQ2 and KCNQ3 was excluded for this family, indicating the existence of an additional locus for seizures occurring in early infancy.

\subsection{Identification of mutations in the sodium channel subunit gene SCN2A}

SCN2A (OMIM 182390) is located at chromosome 2q24.3 and contains 26 exons spread across $150 \mathrm{~kb}$ of genomic DNA. The gene codes for the voltage-gated sodium channel alpha subunit $\mathrm{Na}_{\mathrm{v}} 1.2$, a large protein of 2005 amino acids. Voltage-gated sodium channels consist of the large alpha subunit and two small accessory beta subunits. The alpha subunits consist of a small N-terminal domain, four transmembrane domains (DI-DIV) linked by intracellular loops, and a large C-terminal domain. The transmembrane domains all comprise six transmembrane segments (S1-S6) with a pore-loop between segments S5 and S6. The S4 domains act as voltage sensors. Overall, the structure of one of these transmembrane domains is very similar to the structure of the transmembrane domains of the voltage-gated potassium channels associated with BFNE described above. Indeed, it is thought that the voltage-gated sodium channels evolved from the assembly of four voltagegated potassium channels (Marban et al., 1998). 
SCN2A was originally suggested as a candidate gene for BFIE by Malacarne and colleagues (2001) when they mapped four BFIE families to the chromosome 2q24.3 locus. Mutations in SCN2A were then described in two families: the family described by Lewis and colleagues (1996) and a newly described family (Heron et al., 2002). Mutations have been subsequently described in another ten families and one sporadic case diagnosed clinically with neonatal seizures, who had a de novo mutation (Berkovic et al., 2004; Striano et al., 2006a; Herlenius et al., 2007; Liao et al., 2010). These families included the one originally described by Kaplan and Lacey (1983) and one of the families mapped to the SCN2A region by Malacarne and colleagues (2001). Interestingly, two of these families were originally described by clinical criteria as having BFIE (Malacarne et al., 2001; Striano et al., 2006a), highlighting the clinical overlap between BFNIE and BFIE.

The mutations in SCN2A associated with BFNIE are distributed throughout the SCN2A protein. Most of them occur either in the transmembrane segments or in intracellular loops, as shown in Figure 2. The mutations were originally predicted to increase sodium current, as increased sodium current leads to hyperexcitability (Heron et al., 2002). Functional studies done for six of the mutations supported this prediction (Scalmani et al., 2006; Xu et al., 2007; Liao et al., 2010). There have been two possible mechanisms suggested as the basis for the transient occurrence of the seizures in BFNIE. The first of these is age-dependent alternative splicing of $S C N 2 A$. The second is developmental changes in the expression of the gene. Studies showing a decrease in the expression of the SCN2A gene during development and a change in the intracellular distribution of the $\mathrm{Na}_{\mathrm{v}} 1.2$ protein suggest that the latter mechanism is the more plausible of the two (Liao et al., 2010).

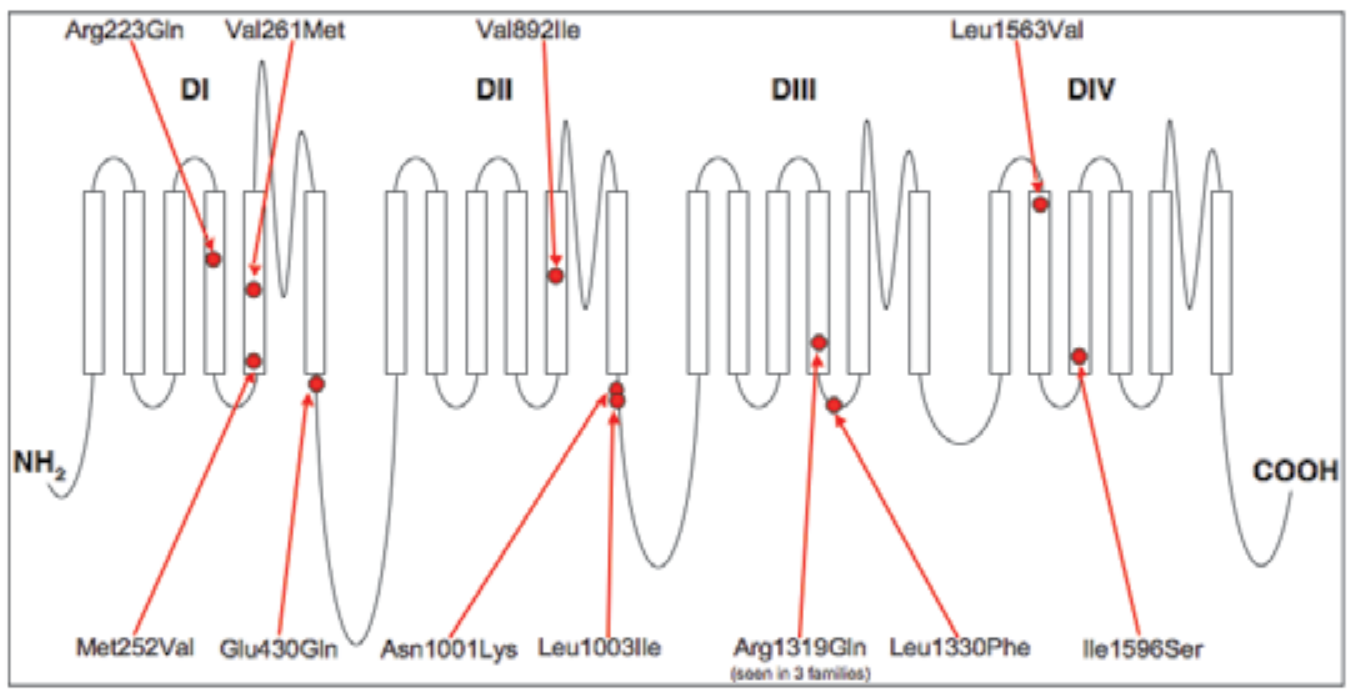

Fig. 2. Diagram showing the structure of the SCN2A protein and the locations of published mutations associated with BFNIE (red circles). The four transmembrane domains are indicated in bold.

\subsection{Clinical overlap between BFNE and BFNIE}

There is considerable similarity in the phenotypes of BFNE caused by potassium channel mutations and BFNIE caused by SCN2A mutations. The epileptic seizures in BFNE and 
BFNIE are similar except for a difference in average age of onset and the two disorders cannot always be distinguished on the basis of age of onset (Mulley et al., 2011). As discussed above, the age of onset in BFNIE ranges from a few days up to one year of age. While most BFNE patients have onset in the neonatal period, occasional patients are reported with later ages of onset up to around 4 months of age, which is the typical age of onset for BFNIE (Singh et al., 2003; de Haan et al., 2006; Heron et al., 2007). A Chinese family with a KCNQ2 mutation has been reported in which the entire family had seizure onset at between 2 and 4 months of age (Zhou et al., 2006). This variation in the age of seizure onset means that, especially in small families or those with limited clinical information, it is difficult to distinguish between BFNE and BFNIE in both familial and sporadic cases.

This clinical overlap means that determining the genetic defect is the only certain method to distinguish between BFNE and BFNIE. Making the distinction accurately is of clinical significance as $15 \%$ of patients with KCNQ2 mutations have seizures in later life, while this is rare in patients with $S C N 2 A$ mutations. Seizures in later life have also not been reported in patients with $K C N Q 3$ mutations, although the total number of patients described with these mutations is small (Hirose et al., 2000; Singh et al., 2003; Li et al., 2008a). Genetic diagnosis therefore has prognostic value in these disorders in the context of predicting the likelihood of later seizures. Severe epileptic encephalopathies have also been reported in families with KCNQ2 mutations and this is another consideration when counselling families with KCNQ2 mutations as opposed to those with SCN2A or KCNQ3 mutations (Dedek et al., 2003; Borgatti et al., 2004; Schmitt et al., 2005; Stenlein et al., 2007). Extension of genetic diagnosis to BFIE awaits the discovery of the BFIE genes.

\section{The molecular genetics of benign familial infantile epilepsy}

Benign familial infantile epilepsy (BFIE) has a later average age of onset than BFNE and BFNIE (Berkovic et al., 2004). The disorder was originally described by Watanabe and colleagues (1987), who observed that a subgroup of infants with generalised motor seizures had subsequent normal development and later became seizure-free. Additional families were described by Vigevano and colleagues (1992) several years later. The disorder was demonstrated to be genetically distinct from BFNE in 1994 when Malafosse and colleagues excluded BFIE families from linkage to the BFNE region containing KCNQ2.

A subset of BFIE patients also have paroxysmal movement disorders with onset in adolescence: either paroxysmal kinesogenic choreoathetosis or paroxysmal dyskinesia. The co-occurrence of these disorders is referred to as infantile convulsions and choreoathetosis (ICCA) syndrome. None of the genes associated with BFIE have been identified. The disorder has been mapped to loci on chromosomes 1, 16 and 19 (Guipponi et al., 1997; Szepetowski et al., 1997; Li et al., 2008b). A single family has been described with partial cosegregation of BFIE and familial hemiplegic migraine (FHM) and a mutation in ATP1A2 (Vanmolkot et al., 2003).

\subsection{Rare BFIE loci}

The first locus for BFIE was described in 1997. Five Italian families were mapped to a locus on chromosome 19q. The five families shared partial marker haplotypes for the region, possibly suggesting a founder effect - that is, that the five families were descended from a common ancestor and had the same mutation. No further families have been described which map to this locus and the mutation in the region has not been identified. Study of a further seven 
Italian BFIE families showed that they were not linked to the locus (Gennaro et al., 1999). This suggests that the putative mutation at the chromosome 19 locus is a rare cause of BFIE restricted to a localised geographic region. Indeed, given the possible founder effect in the five families originally linked to the locus, it may harbour a single BFIE founder mutation.

Another single BFIE family has been mapped to a locus on chromosome 1 . This was a large Chinese family containing eight affected individuals. The family was mapped to a 12.4 centiMorgan region on chromosome 1p36.12-p35.1 ( $\mathrm{Li}$ et al., 2008b) with a maximum LOD score of 3.14. Analysis of 45 candidate genes selected from the 315 genes in the region on the basis of function or neuronal expression did not identify any causative mutations in the family (Li et al., 2010).

\subsection{BFIE associated with ATP1A2}

A single family has been described in which BFIE partially co-segregates with familial hemiplegic migraine (FHM) (Vanmolkot et al., 2003). FHM has been associated with several genes, including ATP1A2, the gene mutated in the family with BFIE. No further families have been described with BFIE and mutations in ATP1A2. However, several families have been described with mutations in the gene and other epileptic phenotypes (Haan et al., 2007). It appears that mutations in ATP1A2 can cause a predisposition to seizures, without being associated with any particular seizure type. Given that there was only partial co-segregation of BFIE and the ATP1A2 mutation in the family described by Vanmolkot and colleagues (2003), it is possible that additional mechanisms are contributing to the seizures in the family.

\subsection{The chromosome 16p11.2-q12.1 BFIE locus}

By far the most common locus for BFIE is located in the pericentromeric region of chromosome 16, between 16p11.2 and 16q12.1. This locus was originally described by Szepetowski and colleagues (1997), who mapped four French families with co-occurrence of BFIE and paroxysmal kinesigenic choreoathetosis (PKC) to the region. Numerous other families have subsequently been mapped to the region for similar conditions. Numerous additional smaller families are consistent with mapping to the region, showing no recombination, although they are of insufficient size to demonstrate linkage. Families mapping to the region include those with BFIE or PKC and PKD alone as well as those with both disorders (ICCA). The intervals for ICCA, BFIE and PKC/PKD from 13 published studies are shown in Figure 3. Two of these localisations (Valente et al., 2000; Callenbach et al., 2005) are small and do not overlap, suggesting that there may be more than one causative gene in the region. However, most of the localisations are large and span the centromere, a region of greatly reduced recombination on chromosome 16, especially in female meioses.

Despite the large number of families that have been mapped to the locus (approximately 50 have been described in the literature), no causative mutation has been identified at the chromosome 16 BFIE locus. A recent analysis of copy number variants in the region did not reveal any pathogenic copy number changes in the region, although low copy numbers of a particular variant were observed with increased frequency in a small cohort of BFIE patients (Roll et al., 2010). This suggests that the BFIE mutation on chromosome 16 has an unusual mechanism or is in an unannotated gene. Such unusual mutational mechanisms include: mutations in regulatory regions; balanced inversions; small copy number changes, which would not be detected by standard CGH arrays; and repeat expansions or contractions as in unstable triplet repeats. 


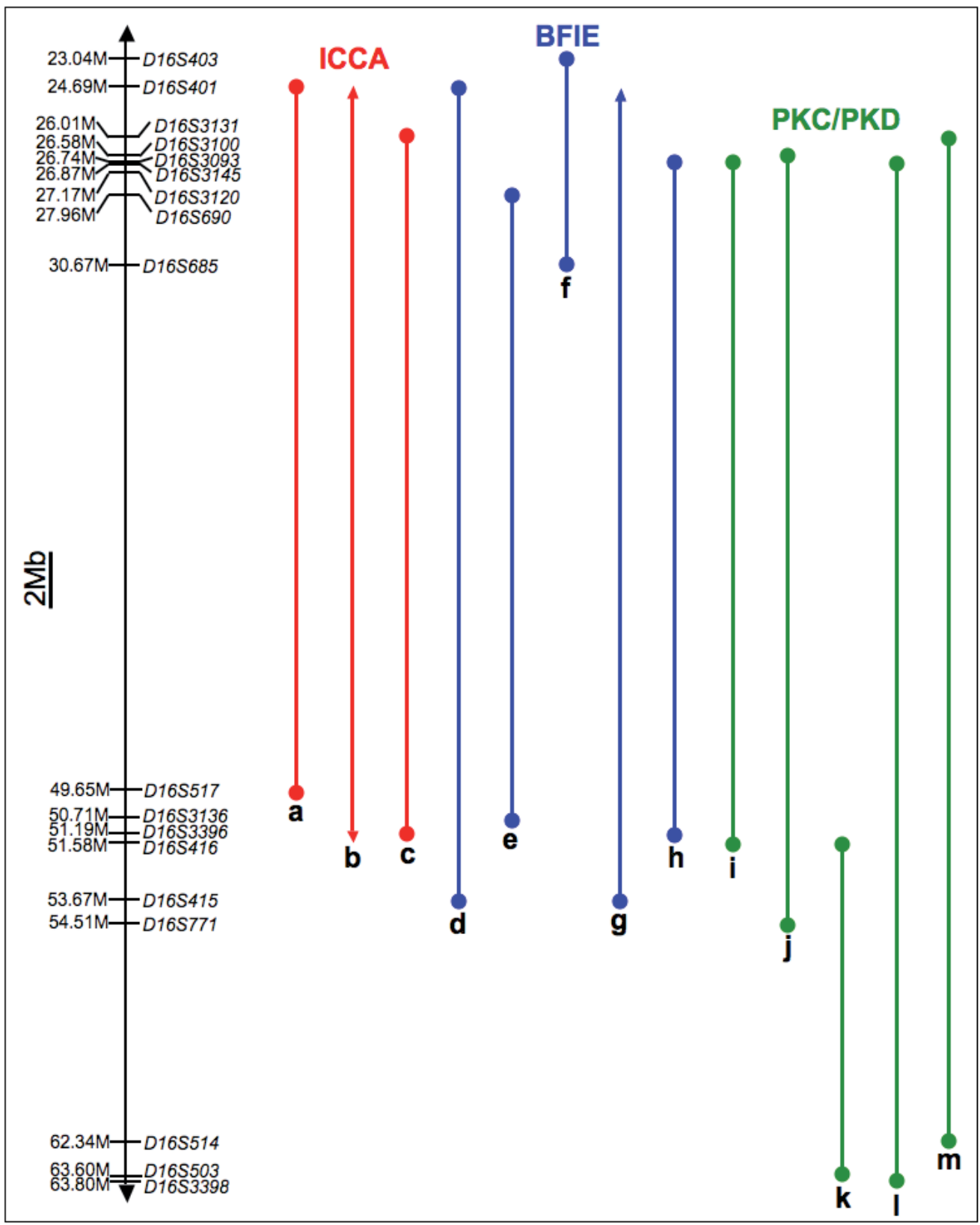

Fig. 3. Physical map showing the positions of microsatellite markers in the pericentromeric region of chromosome 16 and the linkage intervals for ICCA (red), BFIE (blue) and PKC/PKD (green) determined by analysis using the markers. These intervals are derived from the following 13 publications: aSzepetowski et al., 1997; bLee et al., 1998; cSwoboda et al., 2000; dCaraballo et al., 2001; eWeber et al., 2004; fCallenbach et al., 2005; gStriano et al., 2006b; hWeber et al., 2008; iTomita et al., 1999; jBennet et al., 2000; kValente et al., 2000;

lCuenca-Leon et al., 2002; mKikuchi et al., 2007. 
The identification of the mutation in the chromosome 16 BFIE locus will potentially require the application of techniques for the detection of alterations in gene expression, analysis of the non-coding sequence or the identification of small copy number changes. These techniques have not traditionally been applied for mutation detection. However, they may be required for the identification of the mutation or mutations causing BFIE linked to the pericentromeric region of chromosome 16.

\section{Conclusions}

The molecular genetic basis of the benign epilepsies of infancy has not been fully determined. The molecular basis for BFIE remains elusive. However, striking progress has been made in determining the mutations underlying BFNE, of which around $90 \%$ are in the potassium channel subunit gene KCNQ2. A significant proportion of these are exonic deletions or duplications, so in addition to DNA sequencing comprehensive screening for mutations in this gene requires the application of a technique such as multiplex ligation dependent probe amplification (MLPA) for detecting copy number mutations. A small number of BFNE mutations have also been found in the KCNQ3 subunit, which appears to be far less mutable than $K C N Q 2$. It is apparent from the report of a BFNE family with a pericentromeric inversion of chromosome 5 that other, rare, BFNE loci exist.

Some cases of BFNE associated with developmental delay or intellectual disability are caused by duplications of chromosome $2 \mathrm{q} 24.3$. This region contains a cluster of voltage-gated sodium channels and the overexpression of these and perhaps other contiguous genes within the microduplication is hypothesised to cause the seizures and other phenotypes associated with the duplications. Only two cases with these duplications have been described so far, but it is likely that more will emerge with the increased routine application of array comparative genome hybridisation in cases that do not fit within conventional syndrome parameters.

Mutations in the voltage-gated sodium channel SCN2A have been identified as a cause of BFNIE. There is considerable phenotypic overlap between BFNE and BFNIE, which in some cases can only be resolved by identifying a genetic defect in either SCN2A or $K C N Q 2 / K C N Q 3$. This is of clinical significance as patients with neonatal or infantile seizures caused by KCNQ2 mutations have a significantly higher risk of later seizures than patients with SCN2A or KCNQ3 mutations, based on current observations of families that have been studied in detail.

Several loci have been mapped for BFIE, but no genes have been identified at any of these loci. The most important locus located in the pericentromeric region of chromosome 16 has been the focus of considerable research, but no causative gene has been identified as yet. The identification of the BFIE gene on chromosome 16 remains is a major challenge for completing the molecular picture for the benign epilepsies of infancy.

\section{Acknowledgements}

This work was supported by the National Health and Medical Research Council of Australia and SA Pathology.

\section{References}

Bennett, L.B.; Roach, E.S. \& Bowcock, A.M. (2000) A locus for paroxysmal kinesigenic dyskinesia maps to human chromosome 16. Neurology, Vol.54, No.1 (11 th January 2000), pp. 125-130. 
Berg, A.T.; Berkovic, S.F.; Brodie, M.J.; Buchalter, J.; Cross, J.H.; van Emde Boas, W.; Engel, J.; French, J.; Glauser, T.A.; Mathern, W.; Moshé, S.L.; Nordli, D.; Plouin, P. \& Scheffer, I.E. (2010) Revised terminology and concepts for organization of seizures and epilepsies: Report of the ILAE Commission on Classification and Terminology, 2005-2009. Epilepsia, Vol.51, No.4, (April 2010), pp. 676-685.

Berkovic, S.F.; Heron, S.E.; Giordano, L.; Marini, C.; Guerrini, R.; Kaplan, R.E.; Gambardella, A.; Steinlein, O.K.; Grinton, B.E.; Dean, J.T.; Bordo, L.; Hodgson, B.L.; Yamamoto, T.; Mulley, J.C.; Zara, F. \& Scheffer, I.E. (2004) Benign familial neonatal-infantile seizures: characterization of a new sodium channelopathy. Annals of Neurology, Vol.55, No.4, (April 2004), pp. 550-557.

Biervert, C.; Schroeder, B.C.; Kubisch, C.; Berkovic, S.F.; Propping, P.; Jentsch, T.J. \& Steinlein, O.K. (1998) A potassium channel mutation in neonatal human epilepsy. Science, Vol.279, No.5349, (16 ${ }^{\text {th }}$ January 1998), pp. 403-406.

Borgatti, R.; Zucca, C.; Cavallini, A.; Ferrario, M.; Panzeri, C.; Castaldo, P.; Soldovieri, M.V.; Baschirotto, C.; Bresolin, N.; Dalla Bernardina, B.; Taglialatela, M. \& Bassi, M.T. (2004) A novel mutation in KCNQ2 associated with BFNC, drug resistant epilepsy, and mental retardation. Neurology, Vol.63, No.1, (13 th July 2004), pp. 5765.

Caraballo, R.; Pavek, S.; Lemainque, A.; Gastaldi, M.; Echenne, B.; Motte, J.; Genton, P.; Cersósimo, R.; Humbertclaude, V.; Fejerman, N.; Monaco, A.P.; Lathrop, M.G.; Rochette, J. \& Szepetowski, P. (2001) Linkage of benign familial infantile convulsions to chromosome 16p12-q12 suggests allelism to the infantile convulsions and choreoathetosis syndrome. American Journal of Human Genetics, Vol.68, No.3, (March 2001), pp. 788-794.

Callenbach, P.M.; van den Boogerd, E.H.; de Coo, R.F.; ten Houten, R.; Oosterwijk, J.C.; Hageman, G.; Frants ,R.R.; Brouwer, O.F. \& van den Maagdenberg, A.M.J.M. (2005) Refinement of the chromosome 16 locus for benign familial infantile convulsions. Clinical Genetics, Vol.67, No.6, (June 2005), pp. 517-525.

Concolino, D.; Iembo, M.A.; Rossi, E.; Giglio, S.; Coppola, G.; Miraglia Del Giudice, E. \& Strisciuglio, P. (2002) Familial pericentric inversion of chromosome 5 in a family with benign neonatal convulsions. Journal of Medical Genetics, Vol.39, No.3, (March 2002), pp. 214-216.

Charlier, C.; Singh, N.A.; Ryan, S.G.; Lewis, T.B.; Reus, B.E.; Leach, R.J. \& Leppert, M. (1998) A pore mutation in a novel KQT-like potassium channel gene in an idiopathic epilepsy family. Nature Genetics, Vol.18, No.1, (January 1998), pp. 53-55.

Cuenca-Leon, E.; Cormand, B.; Thomson, T. \& Macaya, A. (2002) Paroxysmal kinesigenic dyskinesia and generalized seizures: clinical and genetic analysis in a Spanish pedigree. Neuropediatrics, Vol.33, No.6 (December 2002), pp.288-293.

Dedek, K.; Kunath, B.; Kananura, C.; Reuner, U.; Jentsch, T.J. \& Steinlein, O.K. (2001) Myokymia and neonatal epilepsy caused by a mutation in the voltage sensor of the KCNQ2 K $+\square$ channel. Proceedings of the National Academy Science USA, Vol.98, No.21, (9th October 2001), pp.12272-12277.

Dedek, K.; Fusco, L.; Teloy, N. \& Steinlein, O.K. (2003) Neonatal convulsions and epileptic encephalopathy in an Italian family with a missense mutation in the fifth 
transmembrane region of KCNQ2. Epilepsy Research, Vol.54, No.1, (April 2003), pp. 21-27.

de Haan, G.J.; Pinto, D.; Carton, D.; Bader, A.; Witte, J.; Peters, E.; van Erp, G.; Vandereyken, W.; Boezeman, E.; Wapenaar, M.C.; Boon, P.; Halley, D.; Koeleman, B.P. \& Lindhout, D. (2006) A novel splicing mutation in KCNQ2 in a multigenerational family with BFNC followed for 25 years. Epilepsia, Vol.47, No.5, (May 2006), pp. 851-859.

Delmas, P. \& Brown, D.A. (2005) Pathways modulating neural KCNQ/M (Kv7) potassium channels. Nature Reviews: Neuroscience, Vol.6, No.11, (November 2005), pp. 850-862.

Geiger, J.; Weber, Y.G.; Landwehrmeyer, B.; Sommer, C \& Lerche, H. (2006) Immunohistochemical analysis of KCNQ3 potassium channels in mouse brain. Neuroscience Letters, Vol.400, No.1-2, (29th May 2006), pp. 101-104.

Gennaro, E.; Malacarne, M.; Carbone, I.; Riggio, M.C.; Bianchi, A.; Bonanni, P.; Boniver, C.; Dalla Bernardina, B.; De Marco, P.; Giordano, L.; Guerrini, R.; Santorum, E.; Sebastianelli, R.; Vecchi, M.; Veggiotti, P.; Vigevano, F.; Bricarelli, F.D. \& Zara, F. (1999) No evidence of a major locus for benign familial infantile convulsions on chromosome 19q12-q13.1. Epilepsia, Vol.40, No.12, (December 1999), pp. 17991803.

Goldberg-Stern, H.; Kaufmann, R.; Kivity, S.; Afawi, Z.; \& Heron, S.E. (2009) Novel Mutation in KCNQ2 Causing Benign Familial Neonatal Seizures. Pediatric Neurology, Vol.41, No.5, (November 2009), pp.367-370.

Guipponi, M.; Rivier, F.; Vigevano, F.; Beck, C.; Crespel, A.; Echenne, B.; Lucchini, P.; Sebastianelli, R.; Baldy-Moulinier, M. \& Malafosse A. (1997) Linkage mapping of benign familial infantile convulsions (BFIC) to chromosome 19q. Human Molecular Genetics, Vol.6, No.3, (March 1997), pp. 473-477.

Haan, J.; Terwindt, G.M.; van den Maagdenberg, A.M.J.M.; Stam, A.H. \& Ferrari, M.D. (2008) A review of the genetic relation between migraine and epilepsy. Cephalalgia, Vol.28, No.2, (February 2008), pp. 105-113.

Herlenius, E.; Heron, S.E.; Grinton, B.E.; Keay, D.; Scheffer, I.E.; Mulley, J.C. \& Berkovic, S.F. (2007) SCN2A mutations and benign familial neonatal-infantile seizures: The phenotypic spectrum. Epilepsia, Vol.48, No.6, (June 2007), pp. 1138-1142.

Heron, S.E.; Crossland, K.M.; Andermann, E.; Phillips, H.A.; Hall, A.J.; Bleasel, A.; Shevell, M.; Mercho, S.; Seni, M-H.; Guiot, M.C.; Mulley, J.C.; Berkovic, S.F. \& Scheffer, I.E. (2002) Sodium-channel defects in benign familial neonatal-infantile seizures. Lancet, Vol.360, No.9336, (14th September 2002), pp. 851-852.

Heron, S.E.; Cox, K.; Grinton, B.E.; Zuberi, S.M.; Kivity, S.; Afawi, Z.; Straussberg, R.; Berkovic, S.F.; Scheffer, I.E. \& Mulley, J.C. (2007) Deletions and duplications in KCNQ2 can cause benign familial neonatal seizures. Journal of Medical Genetics, Vol.44, No.12, (December 2007), pp. 791-796.

Heron, S.E.; Scheffer, I.E.; Grinton, B.E.; Eyre, H.; Oliver, K.L.; Bain, S.; Berkovic, S.F. \& Mulley, J.C. (2010) Familial neonatal seizures with intellectual disability caused by a microduplication of chromosome 2q24.3. Epilepsia, Vol.51, No.9, (September 2010), pp. 1865-1869. 
Hirose, S.; Zenri, F.; Akiyoshi, H.; Fukuma, G.; Iwata, H.; Inoue, T.; Yonetani, M.; Tsutsumi, M.; Muranaka, H.; Kurokawa, T.; Hanai, T.; Wada, K.; Kaneko, S. \& Mitsudome, A. (2000) A novel mutation of KCNQ3 (c.925T $\rightarrow$ C) in a Japanese family with benign familial neonatal convulsions. Annals of Neurology, Vol.47, No.6, (June 2000), pp. 822-826.

Ishii, A.; Fukuma, G.; Uehara, A.; Miyajima, T.; Makita, Y.; Hamachi A.; Yasukochi, M.; Inoue, T.; Yasumoto, S.; Okada, M.; Kaneko, S.; Mitsudome, A.; \& Hirose, S. (2009) A de novo KCNQ2 mutation detected in non-familial benign neonatal convulsions. Brain E Development, Vol.31, No.1, (January 2009), pp.27-33.

Kanaumi, T.; Takashima, S.; Iwasaki, H.; Itoh, M.; Mitsudome, A \& Hirose, S. (2008) Developmental changes in KCNQ2 and KCNQ3 expression in human brain: Possible contribution to the age-dependent etiology of benign familial neonatal convulsions. Brain \& Development, Vol.30, No.5, (May 2008), pp. 362-369.

Kaplan, R.E. \& Lacey, D.J. (1983) Benign familial neonatal-infantile seizures. American Journal of Medical Genetics, Vol.16, No.4, (December 1983), pp. 595-599.

Kikuchi, T.; Nomura, M.; Tomita, H.; Harada, N.; Kanai, K.; Konishi, T.; Yasuda, A.; Matsuura, M.; Kato, N.; Yoshiura, K. \& Niikawa, N. (2007) Paroxysmal kinesigenic choreoathetosis (PKC): confirmation of linkage to 16p11-q21, but unsuccessful detection of mutations among 157 genes at the PKC-critical region in seven PKC families. Journal of Human Genetics, Vol.52, No.4, pp. 334-341.

Kurahashi, H.; Wang, J.W.; Ishii, A.; Kojima, T.; Wakai, S.; Kizawa, T.; Fujimoto, Y.; Kikkawa, K.; Yoshimura, K.; Inoue, T.; Yasumoto, S.; Ogawa, A.; Kaneko, S. \& Hirose, S. (2009) Deletions involving both KCNQ2 and CHRNA4 present with benign familial neonatal seizures. Neurology. Vol.73, No.15, (13 th Oct 2009), pp. 1214-1217.

Lee, W.L.; Tay, A.; Ong, H.T.; Goh, L.M.; Monaco, A.P. \& Szepetowski, P. (1998) Association of infantile convulsions with paroxysmal dyskinesias (ICCA syndrome): confirmation of linkage to human chromosome 16p12-q12 in a Chinese family. Human Genetics, Vol.103, No.5, (November 1998), pp. 608-612.

Lee, I.C.; Chen, J.Y.; Chen, Y.J.; Yu, J.S. \& Su P.H. (2009) Benign familial neonatal convulsions: Novel mutation in a newborn. Pediatric Neurology, Vol.40, No.5, (May 2009), pp.387-391.

Leppert, M.; Anderson, V.E.; Quattlebaum, T.; Stauffer, D.; O'Connell, P.; Nakamura, Y.; Lalouel, J. M. \& White, R. (1989) Benign familial neonatal convulsions linked to genetic markers on chromosome 20. Nature, Vol. 337, (16 th $^{\text {February } 1989), ~ p p . ~ 647-~}$ 648.

Lewis, T.B.; Leach, R.J.; Ward, K.; O'Connell, P. \& Ryan, S.G. (1993) Genetic heterogeneity in benign familial neonatal convulsions: identification of a new locus on chromosome 8q. American Journal of Human Genetics, Vol.53, No.3 (September 1993), pp. 670-5.

Lewis, T.B.; Shevell, M.I.; Andermann, E.; Ryan, S.G. \& Leach, R.J. (1996) Evidence of a third locus for benign familial convulsions. Journal of Child Neurology, Vol.11, No.3, (May 1996), pp. 211-214. 
Li, H.; Li, N.; Shen, L.; Jiang, H.; Yang, Q.; Song, Y.; Guo, J.; Xia, K.; Pan, Q. \& Tang, B. (2008a) A novel mutation of KCNQ3 gene in a Chinese family with benign familial neonatal convulsions. Epilepsy Research, Vol.79, No.1 (March 2008), pp. 1-5.

Li, H.Y.; Li, N.; Jiang, H.; Shen, L.; Guo, J.F.; Zhang, R.X.; Xia, K.; Pan, Q.; Zi, X.H. \& Tang, B.S. (2008b) A novel genetic locus for benign familial infantile seizures maps to chromosome 1p36.12-p35.1. Clinical Genetics, Vol.74, No.5, (November 2008), pp. 490-492.

Li, N.; Li, H.; Jiang, H.; Shen, L.; Yan, X.; Guo, J.; Song, Y.; Yang, Q.; Wang, Y.; Li, X.; Xiang, R.; Zi, X.; Long, X.; Hu, Z.; Pan, Q.; Xia, K. \& Tang, B. (2010) Mutation detection in candidate genes for benign familial infantile seizures on a novel locus. International Journal of Neuroscience, Vol.120, No.3, (March 2010), pp. 217-221.

Liao, Y.; Deprez, L.; Maljevic, S.; Pitsch, J.; Claes, L.; Hristova, D.; Jordanova, A.; Ala-Mello, S.; Bellan-Koch, A.; Blazevic, D.; Schubert, S.; Thomas, E.A.; Petrou, S.; Becker, A.J.; De Jonghe, P. \& Lerche, H. (2010) Molecular correlates of age-dependent seizures in an inherited neonatal-infantile epilepsy. Brain, Vol.133, No.5, (May 2010), pp. 14031414.

Malacarne, M.; Gennaro, E.; Madia, F.; Pozzi, S.; Vacca, D.; Barone, B.; dalla Bernardina, B.; Bianchi, A.; Bonanni, P.; De Marco, P.; Gambardella, A.; Giordano, L.; Lispi, M.L.; Romeo, A.; Santorum, E.; Vanadia, F.; Vecchi, M.; Veggiotti, P.; Vigevano, F.; Viri, F.; Bricarelli, F.D. \& Zara, F. (2001) Benign familial infantile convulsions: mapping of a novel locus on chromosome $2 q 24$ and evidence for genetic heterogeneity. American Journal of Human Genetic, Vol.68, No.6, (June 2001), pp.1521-1526.

Malafosse, A.; Leboyer, M.; Dulac, O.; Navalet, Y.; Plouin, P.; Beck, C.; Laklou, H.; Mouchnino, G.; Grandscene, P.; Vallee, L.; Guilloud-Bataille, M.; Samolyk, D.; Baldy-Moulinier, M.; Feingold, J. \& Mallet, J. Confirmation of linkage of benign familial neonatal convulsions to D20S19 and D20S20. Human Genetics, Vol.89, No.1, (April 1992), pp. 59-63.

Malafosse, A.; Beck, C.; Bellet, H.; Di Capua, M.; Dulac, O.; Echenne, B.; Fusco, L.; Lucchini, P.; Ricci, S.; Sebastianelli, R.; Feingold, J.; Baldy-Moulinier, M. \& Vigevano F. (1994) Benign infantile familial convulsions are not an allelic form of the benign familial neonatal convulsions gene. Annals of Neurology, Vol.35, No.4, (April 1994), pp. 479482.

Marban, E.; Yamagishi, T. \& Tomaselli, G.F. (1998) Structure and function of voltage-gated sodium channels. Journal of Physiology, Vol.508, Pt.3, (1st May 1998), pp.647-657.

Miceli, F.; Soldovieri, M.V; Lugli, L.; Bellini, G.; Ambrosino, P.; Migliore, M.; Miraglia del Giudice, E.; Ferrari, F.; Pascotto, A. \& Taglialatela, M. (2009) Neurobiology of Disease, Vol.34, No.3, (June 2009), pp.501-510.

Mulley, J.C; Heron, S.E. \& Dibbens, L.M. Proposed genetic classification for the "benign" familial neonatal and infantile epilepsies. Epilepsia, Vol.52, No. 3, (March 2011), pp.649-650.

Neubauer, B.A.; Waldegger, S.; Heinzinger, J.; Hahn, A.; Kurlemann, G.; Fiedler, B.; Eberhard, F.; Muhle, H.; Stephani, U.; Garkisch, S.; Eeg-Olofsson, O.; Müller, U. \& Sander, T. (2008) KCNQ2 and KCNQ3 mutations contribute to different 
idiopathic epilepsy syndromes. Neurology, Vol.71, No.3, (15 th July 2008), pp.177183.

Raymond, G.; Wohler, E.; Dinsmore, C.; Cox, J.; Johnston, M.; Batista, D. \& Wang, T. (2011) An interstitial duplication at 2q24.3 involving the SCN1A, SCN2A, SCN3A genes associated with infantile epilepsy. American Journal of Medical Genetics Part A, epub ahead of print 17th March 2011, doi: 10.1002/ajmg.a.33929.

Rett, A \& Teubel, R. (1964) Neugeborenenkrämpfe im Rahman einer epileptisch belasten Familie. Wein Klinisch Wochenschr, Vol.76, (1964), pp. 609-613.

Richards, M.C.; Heron, S.E.; Spendlove, H.E.; Scheffer, I.E.; Grinton, B.; Berkovic, S.F.; Mulley, J.C. Davy, A. (2004) Novel mutations in the KCNQ2 gene link epilepsy to a dysfunction of the KCNQ2-calmodulin interaction. Journal of Medical Genetics, Vol.41, No.3, (March 2004), p. e35.

Roll, P.; Sanlaville, D.; Cillario, J.; Labalme, A.; Bruneau, N.; Massacrier, A.; Délepine, M.; Dessen, P.; Lazar, V.; Robaglia-Schlupp, A.; Lesca, G.; Jouve, E.; Rudolf, G.; Rochette, J.; Lathrop, G.M. \& Szepetowski, P. (2010) Infantile convulsions with paroxysmal dyskinesia (ICCA syndrome) and copy number variation at human chromosome 16p11. PLoS One, Vol.5, No.10 (29th October 2010), p. e13750.

Ryan, S.G.; Wiznitzer, M.; Hollman, C.; Torres, M.C.; Szekeresova, M. \& Schneider, S. (1991) Benign familial neonatal convulsions: evidence for clinical and genetic heterogeneity. Annals of Neurology, Vol.29, No.5, (May 1991), pp. 469-473.

Sadewa, A.H.; Sasongko, T.H; Gunadi; Lee, M.J.; Daikoku, K.; Yamamoto, A.; Yamasaki, T.; Tanaka, S.; Matsuo, M. \& Nishio, H. (2008) Germ-line mutation of KCNQ2, p.R213W, in a Japanese family with benign familial neonatal convulsion. Pediatrics International, Vol.50, No.2, (April 2008) pp.167-171.

Scalmani, P.; Rusconi, R.; Armatura, E.; Zara, F.; Avanzini, G.; Franceschetti, S. \& Mantegazza, M. (2006) Effects in neocortical neurons of mutations of the $\mathrm{Na}_{\mathrm{v}} 1.2$ $\mathrm{Na}^{+}$channel causing benign familial neonatal-infantile seizures. Journal of Neuroscience, Vol.26, No.40, (4 ${ }^{\text {th }}$ October 2006), pp. 10100-10109.

Schmitt, B.; Wohlrab, G.; Sander, T.; Steinlein, O.K. \& Hajnal, B.L. (2008) Neonatal seizures with tonic clonic sequences and poor developmental outcome. Epilepsy Research, Vol.65, No.3, (July 2005), pp.161-168.

Singh, N.A.; Charlier, C.; Stauffer, D.; DuPont, B.R.; Leach, R.J.; Melis, R.; Ronen, G.M.; Bjerre, I.; Quattlebaum, T.; Murphy, J.V.; McHarg, M.L.; Gagnon, D.; Rosales, T.O.; Peiffer, A.; Anderson, V.E. \& Leppert, M. (1998) A novel potassium channel gene, KCNQ2, is mutated in an inherited epilepsy of newborns. Nature Genetics, Vol.18, No.1, (January 1998), pp. 25-29.

Singh, N.A.; Westenskow, P.; Charlier, C.; Pappas, C.; Leslie, J.; Dillon, J.; Anderson, V.E.; Sanguinetti, M.C.; Leppert, M.F \& BFNC Physician Consortium. KCNQ2 and KCNQ3 potassium channel genes in benign familial neonatal convulsions: expansion of the functional and mutation spectrum. Brain, Vol.126, No.12, (December 2003), pp. 2726-2737.

Soldovieri, M.V.; Miceli, F.; Bellini, G.; Coppola, G.; Pascotto, A. \& Taglialatela, M. (2007) Correlating the clinical and genetic features of benign familial neonatal seizures 
(BFNS) with the functional consequences of underlying mutations. Channels (Austin), Vol.1, No.4 (July-August 2007), pp. 228-233.

Steinlein, O.; Schuster, V.; Fischer, C. \& Häussler, M. (1995) Benign familial neonatal convulsions: confirmation of genetic heterogeneity and further evidence for a second locus on chromosome 8q. Human Genetics, Vol.95, No.4, (April 1995), pp. 411-415.

Steinlein, O.K.; Conrad, C. \& Weidner, B. (2007) Benign familial neonatal convulsions: always benign? Epilepsy Research, Vol.73, No.3, (March 2007), pp. 245-249.

Striano, P.; Bordo, L.; Lispi, M.L.; Specchio, N.; Minetti, C.; Vigevano, F. \& Zara, F. (2006a) A novel SCN2A mutation in family with benign familial infantile seizures. Epilepsia, Vol.47, No.1, (January 2006), pp. 218-220.

Striano, P.; Lispi, M.L.; Gennaro, E.; Madia, F.; Traverso, M.; Bordo, L.; Aridon, P.; Boneschi, F.M.; Barone, B.; dalla Bernardina, B.; Bianchi, A.; Capovilla, G.; De Marco, P.; Dulac, O.; Gaggero, R.; Gambardella, A.; Nabbout, R.; Prud'homme, J.F.; Day, R.; Vanadia, F.; Vecchi, M.; Veggiotti, P.; Vigevano, F.; Viri, M.; Minetti, C. \& Zara, F. (2006b) Linkage analysis and disease models in benign familial infantile seizures: a study of 16 families. Epilepsia, Vol.47, No.6, (June 2006), pp. 1029-1034.

Swoboda, K.J.; Soong, B.; McKenna, C.; Brunt, E.R.; Litt, M.; Bale, J.F, Jr.; Ashizawa, T.; Bennett, L.B.; Bowcock, A.M.; Roach, E.S.; Gerson, D.; Matsuura, T.; Heydemann, P.T.; Nespeca, M.P.; Jankovic, J.; Leppert, M. \& Ptácek, L.J. (2000) Paroxysmal kinesigenic dyskinesia and infantile convulsions: clinical and linkage studies. Neurology, Vol.55, No.20, (25th July 2000), pp. 224-230.

Szepetowski, P.; Rochette, J.; Berquin, P.; Piussan, C.; Lathrop, GM. \& Monaco, A.P. (1997) Familial infantile convulsions and paroxysmal choreoathetosis: a new neurological syndrome linked to the pericentromeric region of human chromosome 16. American Journal of Human Genetics, Vol.61, No.4 (October 1997), pp. 889-898.

Tomita, H.; Nagamitsu, S.; Wakui, K.; Fukushima, Y.; Yamada, K.; Sadamatsu, M.; Masui, A.; Konishi, T.; Matsuishi, T.; Aihara, M.; Shimizu, K.; Hashimoto, K.; Mineta, M.; Matsushima, M.; Tsujita, T.; Saito, M.; Tanaka, H.; Tsuji, S.; Takagi, T.; Nakamura, Y.; Nanko, S.; Kato, N.; Nakane, Y. \& Niikawa, N. (1999) Paroxysmal kinesigenic choreoathetosis locus maps to chromosome 16p11.2-q12.1. American Journal of Human Genetics, Vol.65, No.6, (December 1999), pp. 1688-1697.

Valente, E.M.; Spacey, S.D.; Wali, G.M.; Bhatia, K.P.; Dixon, P.H.; Wood, N,W. \& Davis, M.B. (2000) A second paroxysmal kinesigenic choreoathetosis locus (EKD2) mapping on 16q13-q22.1 indicates a family of genes which give rise to paroxysmal disorders on human chromosome 16. Brain, Vol.123, No.10, (October 2000), PP. 2040-2045.

Vanmolkot, K.R.J.; Kors, E.E.; Hottenga, J.J.; Terwindt, G.M.; Haan, J.; Hoefnagels, W.A.J.; Black, D.F.; Sandkuijl, L.A.; Frants, R.R.; Ferrari, M,D. \& van den Maagdenberg, A.M.J.M. (2003) Novel mutations in the $\mathrm{Na}^{+}, \mathrm{K}^{+}$-ATPase pump gene ATP1A2 associated with familial hemiplegic migraine and benign familial infantile convulsions. Annals of Neurology, Vol.54, No.3, (September 2003), pp. 360-366. 
Vigevano, F.; Fusco, L.; Di Capua, M.; Ricci, S.; Sebastianelli, R. \& Lucchini, P. (1992) Benign infantile familial convulsions. European Journal of Pediatrics, Vol.151, No.8, (August 1992), pp. 608-612.

Volkers, L.; Rook, M.B.; Dasb, J.H.G.; Verbeek, N.E.; W. Groenewegen, W.A.; van Kempen, M.J.A.; Lindhout, D. \& Koeleman, B.P.C. (2009) Functional analysis of novel KCNQ2 mutations found in patients with Benign Familial Neonatal Convulsions. Neuroscience Letters, Vol.462, No.1, (2nd October 2009), pp.24-29.

Watanabe, K.; Yamamoto, N.; Negoro, T.; Takaesu, E.; Aso, K.; Furune, S. \& Takahashi, I. (1987) Benign complex partial epilepsies in infancy. Pediatric Neurology, Vol.3, No.4, (July-August 1987), pp. 208-211.

Weber, Y.G.; Berger, A.; Bebek, N.; Maier, S.; Karafyllakes, S.; Meyer, N.; Fukuyama, Y.; Halbach, A.; Hikel, C.; Kurlemann, G.; Neubauer, B.; Osawa, M.; Püst, B.; Rating, D.; Saito, K.; Stephani, U.; Tauer, U.; Lehmann-Horn, F.; Jurkat-Rott, K. \& Lerche, H. (2004) Benign familial infantile convulsions: linkage to chromosome 16p12-q12 in 14 families. Epilepsia, Vol.45, No.6, (June 2004), pp. 601-609.

Weber, Y.G.; Geiger, J.; Kämpchen, K.; Landwehrmeyer, B.; Sommer, C. \& Lerche, H. (2006) Immunohistochemical analysis of KCNQ2 potasium channels in adult and developing mouse brain. Brain Research, Vol.1077, No.1, (10th Mar 2006), pp.1-6.

Weber, Y.G.; Jacob, M.; Weber, G. \& Lerche, H. (2008) A BFIS-like syndrome with late onset and febrile seizures: suggestive linkage to chromosome 16p11.2-16q12.1. Epilepsia, Vol.49, No.11, (November 2008), pp. 1959-1964.

Wen, H. \& Levitan, I.B. (2002) Calmodulin is an auxiliary subunit of KCNQ2/3 potassium channels. Journal of Neuroscience, Vol.22, No.18 (22nd September 2002), pp. 79918001.

Wuttke, T.V.; Jurkat-Rott, K.; Paulus, W.; Garncarek, M.; Lehmann-Horn, F.; \& Lerche, H. (2007) Peripheral nerve hyperexcitability due to dominant-negative KCNQ2 mutations. Neurology, Vol.69, No.22, (27th November 2007), pp.2045-2053.

Xu, R.; Thomas, E.A.; Jenkins, M.; Gazina, E.V.; Chiu, C.; Heron, S.E.; Mulley, J.C.; Scheffer, I.E.; Berkovic, S.F. \& Petrou, S. (2007) A childhood epilepsy mutation reveals a role for developmentally regulated splicing of a sodium channel. Molecular and Cellular Neuroscience, Vol. 35, No.2, (June 2007), pp. 292-301.

Yalçin, O.; Cağlayan, S.H.; Saltik, S.; Cokar, O.; Ağan, K.; Dervent, A.; \& Steinlein, O.K. (2007) A novel missense mutation (N258S) in the KCNQ2 gene in a Turkish family afflicted with benign familial neonatal convulsions (BFNC). Turkish Journal of Pediatrics, Vol.49, No.4, (October-December 2007), pp.385-389.

Yum, M.S.; Ko, T.S. \& Yoo, H.W. (2010) The first Korean case of KCNQ2 mutation in a family with Benign Familial Neonatal Convulsions. Journal of Korean Medical Science, Vol.25, No.2, (February 2010), pp.324-326.

Yus-Najera, E.; Santana-Castro, I. \& Villaroel, A. (2002) The identification and characterization of a noncontinuous calmodulin-binding site in noninactivating voltage-dependent KCNQ potassium channels. Journal of Biological Chemistry, Vol.277, No.32 (9th August 2002), pp. 28545-28553. 
Zhou, X.; Ma, A.; Liu, X.; Huang, C.; Zhang, Y.; Shi, R.; Mao, S.; Geng, T. \& Li, S. (2006) Infantile seizures and other epileptic phenotypes in a Chinese family with a missense mutation of KCNQ2. European Journal of Pediatrics, Vol.165, No.10, (October 2006), pp. 691-695. 


\section{Part 3}

Animal Models 



\title{
Audiogenic Seizures - Biological Phenomenon and Experimental Model of Human Epilepsies
}

\author{
Inga I. Poletaeva, Irina B. Fedotova, \\ Natalia M. Sourina and Zoya A. Kostina \\ Moscow State University \\ Russia
}

\section{Introduction}

Animal models that recapitulate human epilepsies with more or less details are believed to be of importance for clinics - for studies of anticonvulsants (Reigel et al., 1986, Dailey et al., 1996, Fedotova et al., 1996, Kosacheva et al., 1998, Ross, Coleman, 2000 et al.), for dissection of molecular and biochemical pathogenesis of epilepsy, and for the search of epilepsy susceptibility genes. The use of audiogenic epilepsy in rodents as the model for anticovulsant activity of different drugs is popular, the PubMed search for the key words combination "anticonvulsant and audiogenic and model" resulted in about 100 citations. Thus the detailed investigation of this type of seizures has great value for iur knowledge concerning the genesis of seizure (and epileptogenesis) in general and their genetic basis in particular.

Apart of its practical importance, the rodent audiogenic epilepsy is the enigmatic biological and genetic phenomenon. Actually the biological significance of high sound sensitivity for rodent survival was never questioned but at the same time its connection with audiogenic epilepsy (AE) was not analyzed from this point of view either. This chapter aims first to introduce several data items concerning investigations of Russian audiogenic prone rat strain (Krushinsky-Molodkina, KM) as they were not fully represented in English literature and second - to discuss the general biological mechanisms of audiogenic seizure. It is well known that the good theory is the best friend of practice. There is some hope that elucidating the origin of animal audiogenic epilepsies will bring us closer to still unsolved problems of seizure states in general. The methods which were used in studies of audiogenic epilepsies marched along with neurobiology methods starting from the middle of XX century. The same is now - new technologies are going to be applied and will be mobilized in future as these valuable models (seizure states developing in response to loud sound) are of great use for medical practice and clinic in particular.

\section{General features of audiogenic epilepsy in KM rat strain and rodents of other genotypes}

The data on increased sound sensitivity in audiogenic rats and mice are known for rather long time (Ross, Coleman, 2000, Semiokhina et al., 2006). Although the anomalies in "sensory part" of acoustic impulses in the brain pathway, promoting the acoustic seizure fit is not the single prerequisite for $\mathrm{AE}$ development. The abnormal biochemical and 
physiological status of central auditory (and other) structures are also the important issues (Garcia-Cairasco, 2002). As several audiogenic rat strains exist, the phenomenon rat AE had been explored in different laboratories.

The audiogenic epileptic fits in rats were noted in Wistar Institute at the beginning of rat breeding program (Ross, Coleman, 2000), while the first observation of mouse audiogenic feature was made by N. Studentsov in I.P. Pavlov laboratory. The instrumental conditioning in mice using loud sound as CS unexpectedly resulted in seizure reaction of animals. In mid-1940-s the AE was noted in DBA/2J strain (Hall, 1947) and was also described in different laboratory mouse strains both inbred (Fuller, Smith, 1953) and outbred. Frings and Frings (1953) selected the albino mouse strain (Frings strains) which further was used later to identify the mass-1 gene (Skradski et al., 2001). The selection experiment was also performed in mice later (Chen, Fuller, 1976) in order to investigate the relationship between "inborn" AE fits and fits induced by priming. In 1948 L. Krushinsky, L. Molodkina and D. Fless from Moscow State University started the selection of rats for high susceptibility to"sound seizures". The data, describing the seizure pattern in rats, selected for audiogenic epilepsy, were first published in Russia in 1949 in the "Advances of Contemporary Biology" (v 28, p. 108-133). In English they became available in 1962, as the translation of L. Krushinsky monograph had been issued (Krushinsky, 1964), and in Krushinsky et al. (1970) paper. Starting from the mid-50-s this new KM (for Krushinsky-Molodkina) strain had been extensively used as epilepsy model and model of catatonic state in pharmacological studies (Semiokhina et al., 2006). At that time authors avoided to describe the genetic aspect of the problem because scientific genetic investigation had been formally suppressed in Russia up to mid 60-s. Later the genetic study of AE inheritance had been performed (Romanova et al., 1993). In 1986-87 the inbreeding of KM strain was initiated, and up to the present the KM rat strain is maintained as inbred (more than 45 generations of inbreeding).

The marked phenotypic similarity is characteristic for audiogenic seizure fit and concomitant phenomena in rat strains selected in Russia, USA, France and Brazil. This includes the specific pattern of seizure stages, audiogenic kindling and postictal catalepsy, brain metabolite levels and their changes after the seizure fit. The phenomenology of audiogenic fit as well as numerous data on so called "priming" procedures in large number of mouse and rat genotypes were extensively presented in the review of Ross and Coleman (2000, see also below).

The brief description of rat audiogenic seizure fit will follow. It should be noted that the audiogenic seizures in mice have in general the pattern similar to that of rats with relatively larger proportion of animal deaths as the result of breath arrest (Tupal, Faingold, 2006). Mice of DBA/2J strain is most well known as demonstrating the AE phenotype with the peak intensity in the third decade of the first postnatal month and the decline of the trait expressivity at the age 40-45 days. Frings mice which were selected at the early 1950-s demonstrate the adult age AE (Klein et al., 2004). Later the audiogenic seizure proneness was discovered in mice of 101/HY strain carrying the mutant locus mut-1 which increase their chromosomes sensitivity to the chemical mutagens (Poletaeva et al., 1996).

\subsection{The audiogenic epilepsy phenotype}

The seizure fit develops according to standard pattern in almost $100 \%$ of KM rats with wild run onset in 2-3 s and the clonic-tonic seizures developing in 7-9 s after the sound onset. Audiogenic seizure starts as the wild run stage, consisting of intense running mixed with high jumps of an animal. Wild run stage could stop and the normal waking state of an 
animal gradually restores. If an animal display the single wild run bout and stopped, thus displaying the low intensity fit score (KM arbitrary unit "1"). Sound-evoked wild running induced the pattern of c-Fos similar to that of full AS in naive rats, this fact confirming the epileptic nature of this early audiogenic fit component (Simler et al., 1994). The "epileptic" nature of this stage was accepted as the fact by Fehr et al. (2004), who named it "clonic run". At the same time two earlier studies demonstrated that the wild-run stage is of "mixed" nature, which include the intense movements and jumps characteristic for avoidance reaction (fleeing from the fearful stimulus) and the involuntary seizure-like movements. The "avoidance" component in wild run stage was demonstrated when rats were provided with the capacity to escape the box in which the sound was presented, although such escape occurred only in the part of animals (Plotnikov, 1963, Fless, Salimov, 1974). The recent data confirm the relationship between these two behavior manifestations. N. Garcia-Cairasco (2002) made the detailed analysis of this problem basing on chemical stimulation data (injections of drugs into the restricted brain regions and analyzing the seizure pattern induced). He claims, in particular, that audiogenic-like seizures induced by chemical manipulations of specific subnuclei of inferior colliculi suggest overlapping of convulsive and aversive responses. In the review of Garcia-Cairasco the comparison was presented of audiogenic seizure pattern between the animals of selected strain WAR and resistant rats with audiogenic fit induced by chemical injections into inferior colliculli and other midbrain structures. This comparison demonstrated both common fit features and the peculiarities inherent for rats of selected strain (Garcia-Cairasco, 2002). As this author notes: "Obvious discrepancies could be derived from the use of genetic strains versus normal animals with acquired audiogenic responses" (Garcia-Cairasco, 2002).

At the same time all AE susceptible rodent strains demonstrated the phenomenon of the two phases of wild run (or "two waves of motor excitation" in Krushinsky terms). In such cases the wildly running rat could stop, rat remained quiet for several seconds and then the second run phase would resume. Although in many cases the AE fit proceeds and wild run is followed first by clonic and then by clonic-tonic and tonic "full" seizure with extension of extremities. After convulsions developed the normal state gradually returns. The postictal state of an animal could be represented by either the cataleptic state development (see below) or by the prolonged motor excitation, which is locomotion bout, different from wild run and which at least in KM strain was poorly analyzed. This type of motor "afterexcitation" could be regarded as the phenotypical equivalent of prolonged abnormally high motor excitability.

\subsection{Audiogenic epilepsy prone strains}

The marked phenotypic similarity is characteristic for audiogenic seizure fit and concomitant phenomena in rat strains selected in Russia, USA, France and Brazil. This includes the specific pattern of seizure stages, audiogenic kindling and postictal catalepsy, brain metabolite levels and their changes after the seizure fit. The phenomenology of audiogenic fit as well as numerous data on so called "priming" procedures in large number of mouse and rat genotypes were extensively presented in the review of Ross and Coleman (2000). The brief historical notes concerning other AE susceptible rat strains will follow. At late 1940-s-begin of 1950s several papers were published on audiogenic rats sensitivity presumably using Wistar rats. Recent evaluations show that about 20-25\% of Wistar rat population develop the audiogenic fit of low intensity (demonstrating mainly wild run stage). The strains GEPR-3 and GEPR-9 (Genetic audiogenic prone rats) were created by 
Albert Picchioni and Lincoln Chin in University of Arizona, USA at late 50-s (see Consroe et al., 1979, Reigel et al., 1988, Jobe et al., 1995). There are also WAS-GAERS rats (Wistar audiogenic epilepsy susceptible rats), selected in Strasbourg (Depaulis et al., 1990) and WAR (Wistar aufiogenic rats), which were bred in San-Paolo, Brazil (Doretto et al., 1996, GarciaCairasco 2002 et al.).

The genetic variability in AE propensity was the basis for the successful selection to the high $\mathrm{AE}$ seizure intensity and made it possible to create the respective AE susceptible rat strains. These strains were founded using at least three laboratory rat populations (outbred strains). In Wistar rats the successful selection for audogenic epilepsy was performed at least three times. These strains were: KM strain (already mentioned above), Wistar audiogenic sensitive rats (the detailed history not described, Simler et al., 1994) and the WAR strain which was extensively investigated by N. Garcia-Cairasco and his colleagues (Doretto et al., 1994, 2003, 2009, Garsia-Cairasco, 2002). Long-Evans rats served as the basic population for the strain of AE prone rats with about $100 \%$ penetrance and high expressivity. This strain had been bred by T. Kalinina and A. Volkova from Moscow Institute of Pharmacology (Russian Academy of Medical Sciences). Unfortunately the strain was lost due to breeding problems and only one paper which describes the experimental data using this strain is available (Surina et al., 2011).. The possibility to breed AE prone rats from Long-Evans population was very informative as this strain previously was reported as being very audiogenic-seizure resistant. The sound-induced seizures in these rats were possible to induce only by means of priming procedure (Ross, Colemann, 1999). The GEPR strains were selected using SpragueDawley population (Jobe et al., 1973, Consroe et al., 1979). Rather rapid selection response for this trait was characteristic for each of these experiments. In each case it resulted in creating the viable and long-lasting laboratory population of AE-susceptible rats with high penetrance and expressivity of this trait. In general, it means that the alleles of loci involved in audiogenic seizure propensity are not deleterious to the general neurological and/or somatic state of the animals. Although the oldest strain - KM (which has now more than 45 inbreeding generations and about 150 generations of selection breeding from 1947) demonstrates rather low fertility at present with the delayed start of reproductive age (3.5-4 months).

Audiogenic seizures were also described in the WAG/Rij rat strain which was initially studied as the laboratory model for the "absence" epilepsy (Kuznetzova et al., 1996). Midzyanovskaya et al. (2004) noted that about one third of WAG/Rij rats develop audiogenic seizure fit in response to loud sound stimulation, although these seizures were of low intensity -the tonic seizure stage was demonstrated rarely. GAERS rats also represent the model for "absence" epilepsy although the degree of relatedness between this strain and audiogenic prone strain of Wistar origin in Strasbourg laboratory (Simler et al., 1994) had not been clarified.

\section{Brain structures involved in the development of audiogenic seizures}

Audiogenic seizure fit initiates as the excitation arrives into the brain stem structures, namely in inferior colliculli. This fact has been extensively demonstrated in GEPR strains (Millan et al., 1988, N'Gouemo \& Faingold, 1998, 1999, Faingold, 1999), as well as in WAR and WAS strains and mice (Willott \& Lu, 1980, Garcia-Cairasco, 2002, Garcia-Cairasco \& Sabbatini, 1991, Simler et al., 1994). Bilateral lesions of inferior collicculi, lateral lemniscus and the connections between these structures blocked audiogenic seizures expression in rats and mice (Garcia-Cairasco, 2002, Browning et al., 1999 Garcia-Cairasco, Sabbatini, 1991, Kesner, 1966, cited by Garcia-Cairasco, 2002). 
The epileptic EEG activity concomitant with AE fit was demonstrated in medulla (Krushinsky et al., 1963, Krushinsky et al., 1970), midbrain, namely PAG and substantia nigra (N'Gouemo \& Faingold, 1998, 1999, Doretto et al., 1994), lateral geniculate bodies (Krushinsky, 1963, Ribak et al., 1994), but not in hippocampus or neocortex (Merill et al., 2005, Moraes et al., 2005, Semiokhina et al., 2006). The latter fact was demonstrated both by lack of EEG epileptic pattern during clonic and tonic seizures and by audiogenic fit being unaffected after cortex ablation and during neocortex inactivation in the course of cortical spreading depression (Semiokhina, 1969). Detailed pattern of AE fit development was described in details for GEPR-3 and GEPR-9 by data on EEG and c-fos expression as well by data on brainstem sections and structure ablations (Ribak et al., 1994, Doretto et al., 1994, 2009). The main structures involved in audiogenic fit development are shown on the scheme (Fig.1).

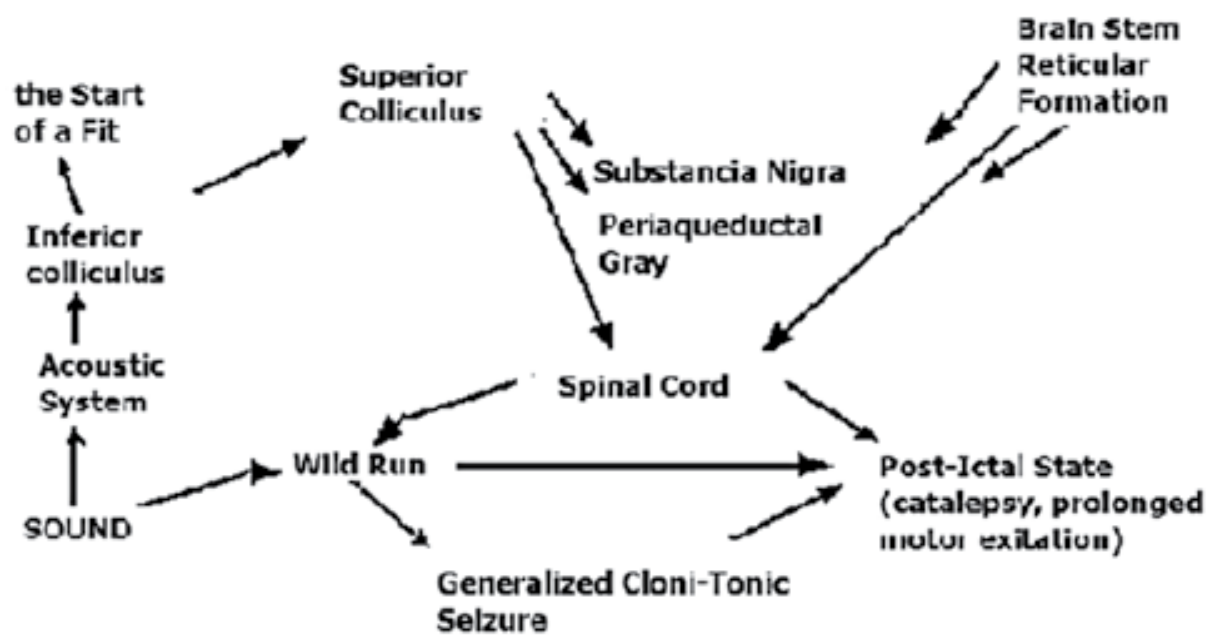

Fig. 1. The schematic representation of audiogenic seizure discharge development involving brain stem structures (see the text)

In several studies of AE, performed on DBA/2J model, the cortical EEG demonstrated the epileptic activity focus (Takao et al., 2006). Although it could be possible that high voltage epileptic potentials could result from electrotonic spread of potentials in the volume conductor, although the active participation of cortical neurons in AE fit development was demonstrated in mice using markers of glia cells activation (Guillaume et al., 1991), demonstrating the cortical catecholamine system activation and suggesting the protective role of neocortex in this process.

Another example of deviations from the commonly accepted view about the lack of EEG activity in the cortex during tonic AE fit was obtained by Zivanovic et al. (1997). The audiogenic seizures induced in non-epileptic Wistar rats by means of metaphit injections (competitive antagonist of NMDA-receptor) manifested the typical epileptic pattern in cortical EEG recordings. Thus the chemically induced AE fits are different by the pattern of pathological discharge propagation from that of "naturally" occurring AE fit in the genetically susceptible rats.

The participation and the key role of inferior colliculi in initiation and development of wild run and motor seizures had been investigated by all techniques available - EEG, activity of 
single neurons (Faingold, 1999), 2-deoxiglucose uptake (Clemmesen et al., 1988) and the investigation the c-Fos expression pattern both in mice (Klein et al., 2004) and rat (Eells et al., 2004). The arrival of excitation to the superior colliculus is the first step in the seizure generalization (Ribak et al., 1997). Ribak \& Morin (1995) showed (using immunocytochemistry and in situ hybridization) the significant increase in GABA level and the larger number of GABAergic neurons in the central nucleus of the inferior colliculus in GEPR-9 strain in comparison to Sprague-Dawley non-epileptic rats. The number of small cells with diameters less than 15 microns showed the greatest increase. The causal connection of this anatomical trait with $\mathrm{AE}$ was proved by the fact that in KM rats (the independent selection experiment from different initial population) the similar increase was recently shown in the number of neurons expressing proteins co-localized with GAD in rat brain of 2, 4 and 6 weeks of age (unpublished data). Ribak \& Morin (1995) using the knife cuts through the midbrain indicated that in sound-induced seizure propagation the important stage was the activation of the inferior colliculus external nucleus via projections from the central nucleus.

It was known for rather long time that audiogenic-like seizures could be evoked by microinjections of bicuculline (GABA-A receptors antagonist) into the inferior colliculi, namely into the central nucleus of this structure (Bagri et al., 1989, Terra \& Garcia-Cairasco, 1992). The more detailed analysis of morphological substrate of wild run stage in rat by means of stimulation as well as by self-stimulation of collicular structures gave similar results. The "wild run" seizures which follow biccuculine microinjections into inferior colliculus and resemble AE fit were never followed by clonic-tonic stage. The infusion of NMDA in this structure had the similar results. Animals displayed more severe spontaneous audiogenic-like seizures after NMDA injection into central ventral or cortical dorsal inferior colliculus nuclei. which were potentiated by the acoustic stimulus. In general these responses resembled the flight reaction. This reaction could be interrupted by an animal only in cases when electrodes were placed into ventral part of central nucleus (of the inferior colliculus), but not in the cortical region of this structure (Garcia-Cairasco, 2002). Most part of these seizure responses developed prior to sound stimulation and they never ended in tonic-clonic seizures. These audiogenic seizures were blocked by microinjections of 2-amino-7-phosphono-heptanoate (AP7) applied just before NMDA microinjections into central or cortical nuclei (Terra \& Garcia-Cairasco, 1994).

The activation of connections between interior and superior colliculli are obligatory for the "sensory-motor transduction in the midbrain", which means that this connection wais activated prior to audiogenic seizure appearance (Garcia-Cairasco, 2002). Thus, the integrity of connections between inferior and superior colliculi projections looks to be the crucial, or "key" point not only for adaptive responses to loud sound (startle, flight) but also for audiogenic seizures (Dutra Moraes et al., 2000). The non-acoustic structures periaqueductal grey, PAG, and substantia nigra, pars lateralis are also critically involved in the network, activated during audiogenic seizure development. The evoked audiogenic-like seizures (by bicuculline application) could not be modified by lesions in the PAG, which suggest that this structure is not necessary for the senso-motor processing of these seizures (Bagri et al., 1991). In contrast, the ventrolateral portion of this structure is involved in the modulation of audiogenic seizures in GEPRs via a complex mechanism mediated by NMDA, GABA and opiates (N'Gouemo \& Faingold, 1998, 1999, Faingold, 1999). In WAR the bilateral microinjections of clobazam into substantia nigra pars reticulata completely blocked mentioned above audiogenic like seizures evoked by unilateral microinjection of 
bicuculline into inferior colliculus (Terra, Garcia-Cairasco, 1992). It made possible to suggest that WARs might be genetically deficient in GABA release or GABA receptors number and/or specificity. In another AE model the block of audiogenic seizures in similar situation was not possible, and the non-involvement of nigro-tectal connections in AE had been suggested (Depaulis et al., 1990).

The study of EEG peculiarities (wavelet analysis) in the striatum - substantia nigra pars reticulate - superior colliculus in WAR strain demonstrated the active participation of this system in audiogenic fit development. It was suggested that this circuitry could probably participate in active seizure fit termination as well as the participation of cerebellum in such process was not investigated in this model. Although the authors claim that methodology employed cannot answer the question - whether the increase of EEG frequency oscillations means the "desinhibition" of a nucleus, or the decrease of EEG frequency means an "inhibition". These data are of rather big importance as the active nature of seizure cessation is still poorly understood (Doretto et al., 1994, Kryzhanovsky, 2002).

The fact that brain stem nuclei are involved in the audiogenic seizure propagation does not necessarily mean that the wild run and tonic convulsions are mediated by the identical neuronal circuitries. For instance two different kinds of glutamate receptors (NMDA and AMPA) were involved in these two stages of the AE fit (Yasuda et al., 1998). The pattern of EEG and init activity in GEPRs permitted to suggest the way by which the excitation spreads in the brain during seizure fit (Dailey et al., 1996, Deransart et al., 2001). Muscimol intranigral inactivating injections were capable to suppress clonic and absence-like states in audiogenic seizure prone Wistars but were not capable to stop the tonic seizure. The inferior collululi seem to be the critical structure as the increase of neuronal firing there precedes the start of the fit. The excitation spread to the deep structures of the superior colluculus plays the similar role for wild run stage as neuronal firing increase also precedes the start of audiogenic fit. When the excitation reaches the pontine reticular nucleus and PAG the clonic-tonic stage of the fit starts (Molnar et al., 2000). After the end of the seizure fit the electric activity in these structures is depressed (dorsal cortex of inferior colliculus being the exception). The existing evidence in GEPR-9 claims that abnormal epileptic activity is determined by the increase of glutamatergic and decrease of GABAergic brain stem systems (Chakravarty \& Faingold, 1999).

\subsection{Priming procedure, hearing defects and audiogenic seizure fit}

Priming procedures, as well as several other treatments (e.g. methaphit injections, Stanojlović et al., 2000) could induce AE in non-susceptible rats and mice, this phenomenon in mice being first analyzed by K. Henry (1967).

Innate susceptibility to audiogenic seizures appears and declines with age at varying rates, depending upon genotype and environmental conditions as well (Henry, 1985). Auditory dysfunctions could be experimentally produced which induce susceptibility in otherwise non-susceptible mice or rats. In order to study the correlation between the cochlear functions and audiogenic seizures in genetically susceptible mice, both measures were obtained from LP/J mice, at ages ranging from 8 to 120 days (Henry, 1985). Susceptibility to sound-produced convulsions was first noted at the age of 12 days, was maximal in the period from 18 to 32 days, and declined rapidly by 40 days, disappearing totally by 120 days of age. Cochlear nerve-evoked potential thresholds were very high at 12 days, were lowest between 18 and 32 days and increased thereafter. The correlation between susceptibility and cochlear thresholds was greatest for high frequencies $(r=-.93)$, intermediate for 
midfrequency $(\mathrm{r}=-.77)$, and poorest for low frequencies $(\mathrm{r}=-.56)$. It was concluded that either genetic or environmental factors which produce an intermediate level of cochlear damage (for high frequency perception) in the young mouse will produce susceptibility to audiogenic seizures (Henry, 1985).

The detailed analysis of "acoustic" behavior and cochlear functions in albino Frings mice, namely in inbred descendants of them was also performed (Henry, Buzzone, 1986). The cochlear action potential thresholds of the susceptible RB/1bg mice were abnormally high, while the resistant inbred RB/3bg mice had normal audiograms of evoked potentials. The F1 hybrid showed heterosis for cochlear function. This RB/1bg line showed little age-related cochlear loss, which probably accounts for its robust sensitivity to audiogenic seizures over most of its lifespan. Earlier studies had demonstrated that the susceptible RB line had a robust evoked potentials, but little or no cochlear microphonic. The susceptible RB/1bg mice had well-defined potentials and cochlear microphonic (Henry, Buzzone, 1986).

GEPRs cochleas hair cell and electrocochleographic alterations, particularly, were investigated (Faingold et al., 1990) being in accordance with mouse data. Furthermore, several neonatal manipulations such as acoustic trauma (Pierson \& Snyder-Keller, 1994) or kanamycin (Pierson \& Swann, 1991), perinatal antithyroid treatment (Middlesworth \& Norris, 1980) induced audiogenic seizure susceptibility in normal rats.

The detailed reviews of $\mathrm{AE}$ after the priming procedure were presented by Ross \& Coleman (2000) and Garcia-Cairasco, (2002). In general, priming is thought to be a disruption in the normal development of activity dependent auditory pathways.

When topography of pure-tone responses in Wistar rat inferior colliculus was mapped (using Fos expression), it was demonstrated, that auditory deprivation, starting at the age of 14 days, as well as neonatal exposure to potentially deafening noise (which resulted in hearing losses) change drastically the topographic frequency representation in inferior colliculus (Pierson \& Snyder-Keller, 1994). As these treatments are known to result in inducing the audiogenic seizure susceptibility, it was believed that this susceptibility might depend on derangements of hearing due to neonatal auditory deprivation. Pierson \& Snyder-Kelly (1994) suggested that the deteriorations in tonotopic organization of inferior colliculi in these cases could be the probable basis of AE. This idea was supported by the fact that the ontogenetic differentiation processes in cochlea and inferior colliculi developed in the similar order - from low to high frequencies. This tonotopic organization was the result of activity-dependent process, and thus the different "priming" treatments which interfere into integrity of normal functional development of inferior colliculus and hearing were effective in AE production.

The hypersensitivity of the inferior colliculus neurons to a high-intensity auditory stimulation and hyperexcitability of these neurons in a stimulation experiment have been reported after priming procedures (Urban \& Willott, 1979; Willott \& Lu, 1980, Sakamoto \& Niki, 2001, cited by Ross, Colemann, 2000). Thus it was more or less established that in majority of cases the AE phenotype could be revealed when inner ear severe damage of different nature took place. Black Swiss mice were audiogenic seizure prone with typical audiogenic fit which peaked in 21 days old animals and declined further (monogenic autocomal-recessive, chromosome 10). The hereditary hearing thresholds increase was found in these mice which were fully developed to the age of 4-5 month. It is interesting to note that this hearing defect was shown not to co-segregate with AE proneness, these two traits being thus independent (Misawa et al., 2002). 
The functional and morphological integrity of the peripheral acoustic organ seems to be very important for development of $\mathrm{AE}$ fits. The structure of mammal cochlea provides the high auditory sensitivity in the broad frequency range (Dallos, 1992).

The mammalian cochlea contains two types of hair cells - inner hair cells and outer hair cells, embedded in a sensory epithelium, which is organ of Corti. Detailed morphology and function of mammalian cochlea was presented in the instructive paper of Dallos (1992). The organ of Corti is placed on the basilar membrane, which is situated along the whole cochlea length being different in firmness from its base to the apex. Basilar membrane moves as the sound vibrations enter the inner ear. Sound signals in vivo are thought to be enhanced by active mechanisms in outer hair cells - they amplify the sound-induced displacements of the basilar membrane (Dallos, 1992). Thus outer hair cells of the mammalian cochlea besides serving as sensory receptors also generate force to enhance auditory sensitivity and frequency selectivity (Mahendrasingam et al., 2010). Inner hair cells function is to relay auditory signals to acoustic nerve endings (which then travel to the brain). Reciprocally, efferent axonal fibers from the medial olivococholear system innervate sensory cells (Ryan et al., 1990). The function of outer hair cells is based on the voltage-dependent contractility of the outer hair cells, which, in turn, depends on pristine, motile protein specified not long ago (Frolenkov, 2006).Prestin is located in the basolateral wall of outer hair cells, and is thought to alter its conformation in response to changes in membrane potential (Mahendrasingam et al., 2010). Prestin is a member of a gene family, solute carrier (SLC) family 26, which encodes anion transporters and related proteins. In humans three genes of this family (SLC26A2, SLC26A3 and SLC26A4) are associated with different human hereditary diseases (Liu et al., 2003). The details of signal transduction and role of the feedback mechanism in the sensitivity of the peripheral acoustic organ could be very important being the part of presumably pathological mechanism of the AE. The medial olivocochlear efferent system is an important component of an active mechanical outer hair cells system in mammals. It seems that no data on this system functional and/or morphological characteristic in audiogenic prone rats or mice exist at the beginning of 2011, although the protective role of medial olive to cochlea in mammalian ear was well described (Christopher \& Smith, 2003).

Morphological and functional changes in auditory path periphery are related to AE in rodents, although this relationship is not simple (Ross \& Coleman, 2000). It could be mentioned, for instance, that igf- 1 (insulin-like growth factor- 1 or somatomedin C) plays the important role in cohlea intrauterine and early post-natal development, although there were no indications that the defects of cochlea development in igf- 1 knockouts are accompanied by audiogenic seizures (Camarero et al., 2001). In mouse mutants - Bronx waltzer (bv/bv) the $75 \%$ loss of inner hair cells cause only moderate elevation of hearing threshold (Schrott et al., 1989). These facts could signify that specific gene-knockout procedure acts selectively inside cochlear cell sparing the neural elements and thus the knockout consequences does not interfere the outer hair cells feedback connections from olive nucleus. At the same time, priming procedures which induced unspecific cochlear damages presumably involving the innervation impairments too, were accompanied by AE. The AE development after priming procedures (early exposure to the loud sound, deafening or toxic kanamicin injections in early postnatal age) does not induce the cochlear damage. So for priming procedure it is necessary and sufficient to change activity in the acoustic neuronal network (e.g. Pierson \& $\mathrm{Li}, 1996)$. 
It should also be mentioned that mapping of fos-like immunoreactive neurons in brain nuclei demonstrated the unexpected decrease of fos expression in the cochlear nuclei and the central nucleus of the inferior colliculi in animals having AE seizures in comparison to controls (Clough et al., 1997)

One of the conclusions which could be drawn on the basis of our present knowledge is that AE develops when acoustic sensitization takes place. Such sensitization could be the result of hereditary inner ear structural (or functional) anomalies of innervation pattern in combination with brain anomalies.

One more source of variability in the function of audiogenic seizure substrate is the level of thyroid hormones, which was reported to influence the AE proneness, although the information was partly controversial. In general the early postnatal onset of hypothyroidism produces audiogenic seizure susceptibility in rodents (Yasuda et al., 1998). These data were supported by developing the audiogenic seizure phenotype in mouse $\mathrm{KO}$ mice lacking specifically TR beta (Thrb (tm1/tm1)) (Ng et al., 2001).

In early 1950s Krushinsky and his colleagues found that the artificial increase of thyroxin levels in KM rats increased the audiogenic fit intensity (Semiokhina et al., 2006). The authors suggested that the elevation of thyroid hormone level increased the general level of CNS excitability thus inducing the fit severity.

The opposite data were reported by Mills \& Savage (1988), who demonstrated, that GEPRs-9 was hypothyroid from the second week of life up to at least 1 year of age.

Using the battery of seven C57 X DBA (BXD) recombinant inbred mouse strains and the D2.B6-Iasb congenic strain, Seyfried et al. (1984) demonstrated in details that there was no genetic correlation of audiogenic seizure susceptibility and the serum thyroxin levels, although in earlier papers the existence of this relationship was more or less obvious as more simple genetic approach was used (Henry et al., 1981).

\subsection{Genetics}

Genetic researches of audiogenic seizures in mice and rats have the separate histories. The identification of Asp loci in DBA/2J mouse strain had been possible on the basis of AE susceptibility scores in F2 hybrids of C57BL.6J (resistant) and DBA/2J susceptible strains (Neumann \& Collins, 1991). The chromosomal location of three Asp (audiogenic seizure proneness) loci was determined by means of classical genetics approach (Fuller et al., 1950). Later J.Fuller performed the new selection experiment creating 4 strains contrasting by initial AE susceptibility and audiogenic seizures induced by priming. The results of new four lines AE testing permitted to conclude that both traits have the independent genetic determination (Fuller, 1975, Chen \& Fuller, 1976).

T. Seyfreid at al. (1980) investigated AE in mice using two parental strains C57BL and DBA mice, their F1 hybrids, backcrosses to both parental strains as well as the battery of 21 recombinant inbred strains. The authors' conclusion was that this physiological trait possessed the hereditary pattern which was characteristic for so called threshold traits. This means that genetic and environmental factors influencing trait in question influence its expressivity but the trait appears as the definite phenotype if the summarized influence of factors exceeds certain "threshold" (Seyfried et al., 1980).

Genetic researches of audiogenic seizures historically bases mainly on data for mouse AE. Three genes were identified on the basis of DBAxC57BL crosses. The first was asp-1 (a major gene, chromosome 12, between Ah and D12 Nyul), the second - asp-2 (with smaller effect, chromosome 4), and the third one - asp-3, which is located in the proximal region of 
chromosome 7 (Neumann \& Seyfried, 1990., Neumann \& Collins, 1992). The authors ascribe the significant role in trait determination to the genomic imprinting. The model, representing these processes, is presented in which the maternal Asp3 allele is repressed, providing an influence largely from the paternal allele (Banko et al., 1997).

The investigations showed the genetic associations of the asp-1 locus with the brain stem Ca 2(+)-ATPase activity level (Neumann \& Seyfried, 1991).

The general seizure susceptibility (maximal electroshock seizure threshold) genes were also investigated by QTL technology using C57BL/6 (B6, seizure resistant) and DBA/2 (D2, seizure susceptible) mice. The data obtained demonstrated a significant effect originating from middle region of chromosome 5. Reciprocal congenic strains between B6 and D2 mice were created by a DNA marker-assisted backcross breeding strategy. Comparison of seizure thresholds between congenic strain and that one which contain the parental genetic background genes indicated that mice from strains having chromosome 5 alleles from D2 and B6 genetic background exhibited significantly lower thresholds, than control littermates, and v.v. - congenic mice harboring B6 chromosome 5 alleles on a D2 genetic background exhibit significantly higher thresholds (Ferraro et al., 2007). Thus that was another chromosome locus which could be also responsible for AE proneness differences between DBA/2J and C57BL/6J mice. Similar data were got using chemically induced seizures, demonstrating the plausible connection between audiogenic seizures and seizures induced by the benzodiazepine receptor inverse agonist methyl-beta-carboline-3-carboxylate (betaCCM). As the injections of this drug induce seizures and the susceptibility for it had the genetic component, both traits were analyzed using recombinant congenic strains of mice bred from B10.D2 and DBA/2J. Although both types of seizures have similar behavioral patterns and might involve GABAergic mechanisms, no correlation was observed between the occurrences of the two types of seizures across the strains, suggesting that these two types of seizures depend on different genetic mechanisms (Martin et al., 1992). These data could be in contrast to the generally increased seizure susceptibility in audiogenic rats (see below).

Another gene had been identified in Black Swiss mice (Misawa et al., 2002), which develop the audiogenic seizure with the pick intensity at 21 postnatal day. Genetic mapping and linkage analysis of hybrid mice localized the gene, jams1 (juvenile audiogenic monogenic seizures), to the region of chromosome 10, delimited by the gene basigin (Bsg) and marker D10Mit140. It is worth to note, that the majority of this critical region is syntenic to a human chromosome 19p13.3 region, which is implicated in a familial form of juvenile febrile convulsions (Klein et al., 2005).

In Frings mice the single gene responsible for $\mathrm{AE}$ was mapped on mouse chromosome 13 (Skradski et al., 2001). This locus was named monogenic audiogenic seizure-susceptible (mass1). The protein coded by mass1 (now referred to as Mgr1) is unique in that it is one of only two identified seizure loci that are not associated with an ion channel mutation. This gene codes for very large G-protein-coupled receptor-1 (vlgr1) which contains about 6300 amino acids, and which is the largest known cell surface protein. It is expressed at high levels within the embryonic nervous system, especially in the ventricular zone. A naturally occurring nonsense mutation in this gene - V2250X, was shown to be linked to susceptibility to audiogenic seizures in mice (McMillan \& White, 2004). Vlgr1d and Vlgr1e - alternativelyspliced variants of Vlgr1b/MGR1 - were shown to be transcripts from a locus mass1. Experiments performed suggested that Vlgr1d and Vlgr1e are secretory molecules, while Vlgr1b is a receptor. Knockout mice lacking exons 2-4 of Vlgr1 were susceptible to 
audiogenic seizures without priming with any apparent histological abnormalities in their brains (Yagi et al., 2005). Mice of other genotypes possessing the Frings Mgr1 allele exhibited a mild to moderate level of hearing impairment which was already present during the days following hearing onset in ontogeny (Klein et al., 2005).

The heritability of audiogenic seizure in rats was no less simple issue. In KM rats the diallelic cross showed that AE heritability is polygenic with additive effects, and alleles determining the resistance to sound induced seizures were dominant (Romanova et al., 1993).

The data on the genetic experiments in other AE rat strains are the following. Audiogenic seizure predisposition in GEPRs was inherited as dominant polygenic autosomal trait (Kurtz et al., 2001). The penetrance and expressivity of the trait was evaluated in 20,373 animals of GEPR strains. The GEPR-3s and GEPR-9s animals both showed incomplete penetrance and variable expressivity of the underlying genetic predisposition to $\mathrm{AE}$. The GEPR-9 strain had more animals with the variable levels of seizure predisposition (as measured by a scoring system that denotes the severity of generalized tonic/clonic seizures) and a greater percentage of animals that exhibited no susceptibility to such seizures induced by sound. Both strains had a number of animals that were not susceptible to sound-induced seizures and that exhibit some variability in seizure severity. The GEPR-9 males show greater differences in expressivity and penetrance compared to GEPR-9 females. The GEPR3 animals also show sex-associated variable penetrance and expressivity of the epileptic phenotype, although the differences are much smaller. It should be noted that in contrast to GEPRs the trait penetrance and expressivity in rats of KM strain were very high - reaching the level of about 99\% (Semiokhina et al.., 2006), probably the different genetic background (Wistar versus Sprague-Dowley) could be responsible. It should be remembered that the KM strain is inbred and display breeding difficulties as well.

Practically no published data could be found concerning the inbreeding used in GEPR strains, while in WAR strain the inbreeding started in parallel with the selection for high AE fit intensity (Garcia-Cairasco, 2002). The selection for high AE predisposition in WAR strain was rather quick (from 3 to $17^{\text {th }}$ generations) the intensity of the fit increasing twice in parallel with fit latencies significant reduction (Doretto et al., 2003). This indicates the additive type of gene action and relative low effect of non-allelic interactions, which resemble the type of $\mathrm{AE}$ inheritance in $\mathrm{KM}$ strain.

The new experiment is now in progress in which the rats were selected for the lack of audiogenic seizures and the basic population was the $\mathrm{F} 2$ and backcross hybrids $\mathrm{KM} x$ Wistar. The " 0 " sensitivity rats which are under selection in this strain share the portion of KM genotype (which is presumably different from the Wistars after more than 100 generations of separate breeding).

The selection success in first ten selection generations of this selection experiment is demonstrated in fig 2. The percentage of rats, demonstrating " 0 " reaction (which was verified by sound exposure for 2-3 times with no less than one week intervals) was never very high, being about $40 \%$ in average. The more thorough analysis performed at the initial stages of selection demonstrated that the distribution of audiogenic sensitivity in F2 and backcrosses corresponds significantly to the two-gene-incomplete penetrance model (Fedotova et al., 2005).

The spontaneous mutation in the rat Wwox gene (Ide/Ide rats) induced dwarfism, postnatal lethality, male hypogonadism, as well as high incidence of epilepsy, typical audiogenic seizures as well, and many structural cell anomalies in the hippocampus and amygdale (Suzuki et al., 2009). 


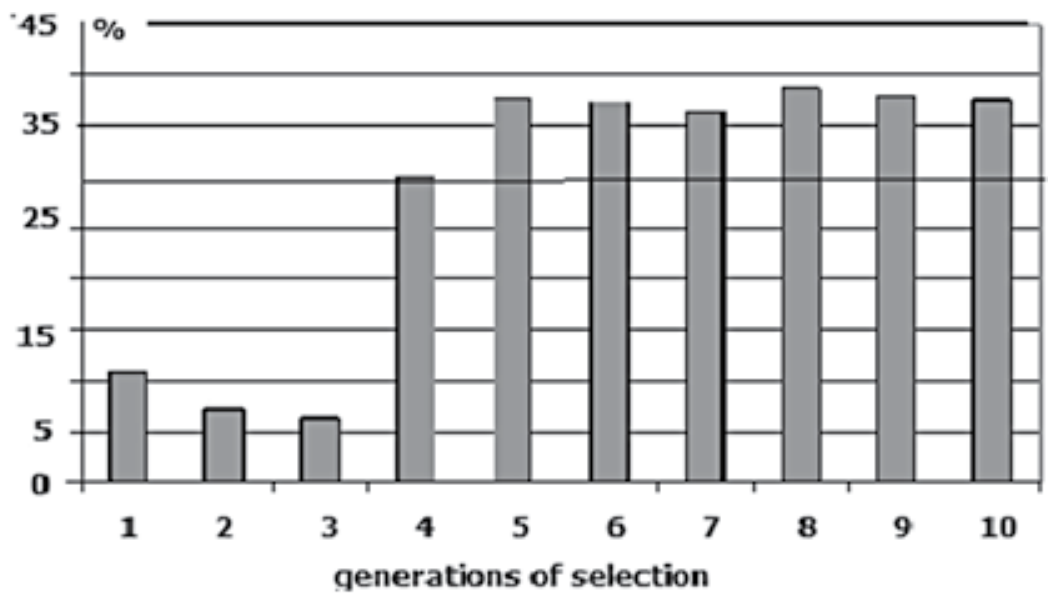

Fig. 2. The percentage of rats, selected for " 0 " audiogenic seizure susceptibility, starting from hydrids between Wistar rats with " 0 " susceptibility and KM rats with maximal seizure intensity. Ordinate - the proportion of " 0 " rats in respectiove generations.

The new data in the field had been brought by new technologies used. $\mathrm{KO}$ of several mouse genes resulted in the audiogenic seizure phenotype - fmr-1, interleikin-6, 5-HT2c receptor (Chen, Toth, 2001, Pacey et al., 2009, Brennan et al., 1997, De Luca et al., 2004). Probably these data are only the start point of new discoveries in this area using gene engineering techniques.

\subsection{Channelopathies}

Seizure states including epilepsy are determined by brain cells pathologies which induce functional changes in neurons. It was demonstrated that in the majority of cases both epileptic neuronal discharges and motor seizures are determined by the deteriorations in ion channels structure and/or function, which were named channelopathies (Mulley et al., 2003, Heron et al., 2007). The changes in the expression of the gene coding for one of the subunits of calcium channel Cav3.2 (as the response to pilocarpin) provoke the anomalous increase of neuronal discharges (Becker et al., 2008). It was demonstrated that in GERPs the dysfunction of voltage-gated $\mathrm{Ca}^{2+}$ channels are of primary importance in the development of audiogenic seizures (N'Gouemo \& Morad, 2003, N'Gouemo et al., 2009, 2010). In the GEPR strains $\mathrm{Ca}^{2+}$ channel blockers (L-type) suppressed the audiogenic fits. The authors demonstrated this dysfunction in neurons of inferior colliculi in GEPRs, while it was unclear whether this "channelopathy" is also characteristic for other brain regions involved in AE. It stayed still unclear whether these $\mathrm{Ca}^{2+}$ current properties are inherent to other $\mathrm{AE}$ genotypes, although the general similarities between different $\mathrm{AE}$ models suggests that this could be the case. $\mathrm{Ca}^{2+}$ currents are also known to activate $\mathrm{K}^{+}$current that initiate the repolarization of action potential and generate after-hyperpolarization potentials. Such $\mathrm{Ca}^{2+}$ activated $\mathrm{K}^{+}$channel may represent an intrinsic inhibitory mechanism that would restore the resting state and maintain normal physiological excitability. The anomalies in this "membrane" trait could be the cause of increased AE susceptibility in rodents. It was demonstrated, that the molecular basis for the enhanced current density of L- and R-type of HVA $\mathrm{Ca}^{2+}$ channels in the GEPR inferior colliculli neurons was the upregulation in the expression of $\mathrm{Ca}^{2+}$ channel a1D and a1E subunits, and it could contribute to the genetic basis of GEPR enhanced seizure susceptibility. The up-regulation of $\mathrm{Ca}^{2+}$ channel a1A subunits 
induced by seizures which was demonstrated, could contribute to the increased neuronal excitability in inferior collicullus. This trait developed after repetitive seizures in the GEPR (N'Gouemo, Morad , 2003, N'Guemo et al., 2010).

\subsection{Myoclonic seizures induced by repetitive audiogenic seizures}

The general notion of brain "kindling" describes the gradual increase of electric responses in limbic system (e.g. hippocampus or amygdala) to the initially subthreshold electrical (or chemical) stimuli (see Galvis-Alonso et al., 2004). Audiogenic kindling (myoclonic seizures, which develop after numerous daily sound exposures) is the result of the spread of seizure discharge to forebrain structures (hippocampus, amygdale, neocortex) after such repetitive stimulation and induces seizure fits of another type. The phenomenology of audiogenic kindling in KM strain was first described by A. Semiokhina in Russian paper (1958, cited by Krushinski, 1963). These seizures are represented by the rhythmic convulsive jerks which involve facial muscles, neck muscles and, as their highest intensity, the musculature of the whole body. In KM rats these seizures appear in response to sound exposure after 10-15 daily sound exposures. These type of seizure (audiogenic kindling) was described and studied extensively in GEPR, WAR and WAS rats (Doretto et al., 2009, Garcia-Cairasco, 2002, N'Gouemo \& Faingold, 1996, Dutra Moraes et al., 2000, Ross \& Coleman, 2000, Simler et al., 1994). Myoclonic seizure fits severity increases in both GEPR substrains and resulted in prominent cortical epileptiform EEG activity (Feng et al., 2001). The same feature was described in KM rats (Semiokhina et al., 2006).

The audiogenic kindling phenotype in GEPRs seems to be different from that observed in KM strain (N'Gouemo \& Faingold, 1996), although War and KM kindling patterns seem to resemble one another (Dutra Moraes et al., 2000). These facts could be ascribed to genetic background similarity/dissimilarity in KMs and Wars from one side and GEPRS - from another.

It appears evident thus, that two factors - the audiogenic seizure proneness and the audiogenic fit "experience" after repetitive sound exposures-form the basis for audiogenic kindling development. The involvement of forebrain structures in the kindling development was demonstrated by EEG techniques as well as by means c-fos expression mapping (Doretto et al., 2009, Garcia-Cairasco, 2002, Eells et al., 2004, Ross \& Coleman, 2000, Semiokhina et al., 2006,). The comparison of brain c-fos expression patterns which was characteristic for audiogenic seizure fit and for audiogenic kindling (mioclonic seizures) demonstrated that during kindling procedure the new process takes place - the "transformation" of midbrain seizure fit into forebrain seizure state. The latter involve the structures which initially were not connected directly with overt motor function (Eells et al., 2004). This process was determined by changes in the neuronal excitability in the genetically abnormal brain of AE susceptible rats.

The special interest was paid to the amygdala participation in audiogenic kindling phenomenon. Bilateral microinjection of a GABA(A) agonist, muscimol into amygdale $(0.3$ nmol/side) significantly reduced the duration of kindling clonic seizures, and they were suppressed by histamine injections. At the same time wild running and tonic components of $\mathrm{AE}$ fit were never affected by microinjection of these agents into the amygdala. Thus amygdala becomes critical for seizure development after the expansion of the seizure network (audiogenic kindling), and this type of seizures were negatively modulated by increased GABA function in this structure (Feng et al., 2001). It was also noted that severe brainstem seizures prevent the behavioral expression of forebrain (kindled) seizures in 
GEPR-9s, although the EEG spike-wave discharges in the forebrain were present. The authors suggested that it was the high intensity of brainstem seizures which did not allow the forebrain seizure to be manifested (Merrill et al., 2005).

In an effort to identify genes involved in molecular mechanisms underlying acute and kindled audiogenic seizures, the suppression-subtractive hybridization was used in order to construct normalized cDNA library enriched for transcripts expressed in the hippocampus of WARs (Gitai et al., 2010). The most represented gene among the 133 clones sequenced was the ionotropic glutamate receptor subunit II (GluR2), a member of the alpha-amino-3hydroxy-5-methyl-4-isoxazoleopropionic acid (AMPA) receptor. The hippocampal levels of the GluR2 subunits do not differ between naïve WARs and their Wistar controls, while the transcript encoding the splice-variant GluR2-flip expression was increased in the hippocampus (namely in CA1 region) of WARs submitted to both acute and kindled audiogenic seizures (Gitai et al., 2010).

Audiogenic kindling is the phenomenon inherent to rats with elevated AE predisposition, and for those which are predisposed genetically. Although the special analysis of the genetic basis of audiogenic kindling in KM rats was performed using the genetic selection. L. Romanova selected KM rats for quick and slow audiogenic kindling development. This selection was successful and the maximum selection effect in both new substrains was achieved in 4-5 generations. This made it possible to suggest that the predisposition for audiogenic kindling development is the oligogenic trait (Romanova et al., 1993).

In mouse no data exist about the possibility to induce the audiogenic kindling. Although in $\mathrm{C} 3 \mathrm{H}$ male mouse brain, which was kindled by electric stimulation of hippocampus differentially expressed genes were screened in various time intervals after kindling. 50 from about 30,000 bands obtained were displayed differentially. Differential expression of genes identified was demonstrated in hippocampus and forebrain, but not in brainstem or cerebellum (Liang \& Seyfried, 2001).

\subsection{Audiogenic postictal catalepsy}

In KM rats as well as in WARs (Garcia-Vairasco, personal communication) the audiogenic seizure fit is followed by the cataleptic state (Semioknina et al., 2006), which was named audiogenic postictal catalepsy. Catalepsy is characterized by the areflexia and drastic changes in the pattern of muscle tone - the so-called waxy flexibility. The duration of postictal audiogenic catalepsy correlated positively with seizure fit intensity (Fedotova et al., 2008). The pharmacological study as well as comparison of catalepsy intensity in rats of different genotypes permitted to conclude that the dopaminergic system was the key structure for this anomaly while glytamate- and GABA-ergic systems participate in its genesis as well playing the roles of modulators. It was possible to "disentangle" audiogenic fit and catalepsy correlation when glutamatergic agents (D-serine and MK-801) were injected (unpublished data). As catatonic syndrome in humans share several features with rat catalepsy we suggest that postictal audiogenic catalepsy could serve as valid laboratory model of this pathology, which would permit to make the preclinical anti-cataleptic drug testing (Fedotova et al., 2008).

\subsection{Brain vascular anomalies}

Early experiments in L.V. Krushinsky laboratory demonstrated that in cases when the rat was exposed to the sound stimulation for the longer period (e.g. ten minutes or more with special pattern of sound "offs" and "ons") the brain vascular disturbances could be noted in 
many cases sometimes with lethal outcome (Krushinsky, 1962, 1963, Krushinsky et al., 1970). The causes of these deaths were the acute brain hemorrhages both epidural and ventricular, which were provoked by drastic changes in the permeability of brain capillary and erythrocyte diapedesis.

In rats which survived the prolonged sound exposure the less serious brain hemorrhages develop as well, which were verified morphologically. These hemorrhages manifested in vivo as hind limbs paresis after sound exposure which restored in several minutes.

The increase in arterial blood pressure was described as the initial phase of audiogenic fit which was followed by its' decrease (up to 87-90 mm Hg) (Krushinsky, 1963). These AP changes are in parallel with capillary permeability anomalies mentioned above. Changes in systemic and regional hemodynamic during sound-induced convulsions were measured in KM rats with microsphere technique. Blood pressure increased from 103 till $178 \mathrm{~mm} \mathrm{Hg}$ and cardiac index rose from 27.3 to $49.3 \mathrm{ml} / \mathrm{min} / 100 \mathrm{~g} \mathrm{~b}$. w. during convulsion stage. Blood flow was increased in the brain and in the heart by $140-700 \%$, whereas in most of internal organs it was decreased by $40-94 \%$ (Ivashev et al., 1991)...

The venous tone was estimated by means of circulatory filling pressure during a short arrest of circulation by inflating a balloon in the right atrium. It was confirmed that, during audiogenic seizure, AP raised from 104 to $156 \mathrm{~mm} \mathrm{Hg}$ on the average, while mean circulatory filling pressure decreased from 8.9 to $7.4 \mathrm{~mm} \mathrm{Hg}$. The intensity of subdural and subarachnoidal hemorrhages correlated with the raise of AP during seizure. The hemorrhagic area spread over $75 \mathrm{~mm}^{2}$ when the AP elevated above the $200 \mathrm{~mm} \mathrm{Hg}$ level, while in lower increment it was only $2.56 \mathrm{~mm}^{2}$ (Vlasov et al., 1991).

The rates of local cerebral blood flow during audiogenic seizures in Wistar audogenic seizure rats belonging to a genetic strain selected for $\mathrm{AE}$ at the Centre de Neurochimie (Strasbourg, France) were analyzed. During single audiogenic seizures, rates of local cerebral blood flow increased over control values in almost all brain areas, the highest increases being in brain stem (inferior and superior colliculus, reticular formation, and many thalamic and hypothalamic regions). The lowest increases were in forebrain limbic regions and cortical areas. In kindled rats the increase of cerebral blood flow was of lower amplitude, than in controls. Thus the good correlation between the involvement of structures in the AE progress and the increase of blood flow in them was discovered. The circulatory changes in kindled rats consisted in the decrease of blood flow, this fact reflecting the differences in respective mechanisms of brain stem $\mathrm{AE}$ seizures and audiogenic kindling (Nehlig et al., 1995).

In KM rats the inhibitor of NO-synthase (L-NNA) increased the severity of seizures, mortality rate and intracranial hemorrhages. In contrast, L-arginine (which is the substrate for NO synthesis) elevated the resistance of animals to acoustic stress. Preliminary exposure of rats to hypoxic conditions (1 hour in hypobaric chamber, simulated altitude of $5000 \mathrm{~m}$ above the sea level) decreased the development of dysfunctions induced by acoustic stress. At the same time the protective effect of hypoxic "training" disappeared after the administration of NO-synthase inhibitor (L-NNA). The study demonstrated that nitric oxide (NO) participates in adaptive reactions of cerebral hemodynamics linked with the significant increase of cerebral blood flow (Fadiukova et al., 2005).

\subsection{Neurochemical correlates of audiogenic seizure susceptibility and fit development}

Brainstem and forebrain neurochemical systems in GERPs and KM rats (to lesser extent) have been characterized. In brief, audiogenic seizure proneness in rats and mice is 
accompanied by the changes in the background neurotransmitters levels, including both the background level and seizure aftereffects. The neurotransmitter systems involved in the generation of AE fits include noradrenergic, dopaminergic, serotoninergic systems, as well as glutamatergic and GABA-ergic neurons and neuronal networks (Laird et al., 1984, Jobe et al., 1986, Ribak et al., 1988, Lasley, 1991, Fedotova et al., 1996, Kosacheva et al., 1998). Fig 3 demonstrates the neurotransmitter aminoacid levels in brain regions of KM strain.

Jobe et al. (1986) found the "reciprocal relationship" between both noradrenergic and serotonergic transmissions and the severity of audiogenic seizures, thus indicating the deficit of brain monoamines as one of the concomitant neurochemical features of AE. The serotoninergic brain system participates in $\mathrm{AE}$ of DBA/2J mice. Interesting enough was the fact that the number of 5 -HT2 binding sites was $20 \%$ higher in the cerebral cortex of DBA/2J in comparison to C57 BL/6 mice at the age of "audiogenic" susceptibility of DBA/2J mice but did not differ at other ages. There were no differences in these parameters between the two strains in forebrain, mid-brain, hippocampus and pons-medulla (Jazrawi et al., 1989).

The failure to replicate previously reported significant differences between the susceptible (DBA/2J) and nonsusceptible (C57BL/6J) mice in the brain monoamine levels at the age of peak seizure susceptibility (in DBAs) demonstrated the difficulties of the whole issue, which was probably due to different techniques used (Lints et al., 1980). The cordotomy and brain monoamine levels approach proved, as authors claimed (Willott et al., 1979), that NE and 5HT are not responsible for attenuation of audiogenic seizures. These data demonstrate that inter species differences (mouse genotypes compared) could be responsible for differences in neurochemical changes during AE fit.

Compared to controls, GEPR-3s and GEPR-9s had a modest and larger increase respectively in Bmax for both high and low affinity GABA sites, with no change in $\mathrm{Kd}$. Chloridedependent, barbiturate-enhanced GABA binding (increased Bmax) was observed for all conditions and groups. Likewise benzodiazepine binding (Bmax) increased slightly in GEPR-9 animals. There were no observed changes in binding sites for a survey of biogenic amines. Seizure-prone animals appeared to have compensatory denervation-like supersensitivity for their most prominent inhibitory receptor, which may or may not be linked to the seizure event (Booker et al., 1986). This fact was also demonstrated in the direct experiments by chronic depletion of brain 5-HT by i.c.v. administration of 5,7dihydroxytryptamine. This procedure induced the significant increase in seizure severity, which could be noted in 2, 3 and 4 weeks after drug injections (as compared to vehicleinjected controls) (Statnick et al., 1996).

The thorough comparison of serotoninergic system was performed by Bakhit et al. (1982). Tryptophan hydroxylase activity (the rate limiting enzyme of serotonin synthesis) was significantly lower in brains of Frings mice compared to CF1 control mice probably due to altered kinetic characteristics of this enzyme. However, brain levels of serotonin were similar in both strains, as well as the uptake of tryptophan and accumulation of 5-HT (following MAO-inhibitor pargyline infusion). These data represent the information suggesting that shifts in brain monoamine levels are not the direct cause of AE seizures (Bakhit et al., 1982).

In $\mathrm{KM}$ rats the diazepam binding in synaptic membrane of different brain regions was significantly lower than in control non-epileptic Wistars (with highest differences being in cerebellum (Joulin \& Pleskacheva, 1991). GABA binding in the cerebellum (but not in the brain stem) was also lower in KM. Presumably the receptor numbers differences but not receptor affinity were the cause of data obtained. Low scores of cerebellum synaptic 
membranes binding should be analyzed in the framework of brain anticonvulsive system concept (Kryzhanovskil., 2002), as this structure is suggested to be central in the process of seizure suppression.

Data of the opposite "sign" were obtained in mice by Robertson (1980). The specific binding of 3H-flunitrazepam was higher in the seizure-susceptible DBA/2J strain (at about 22 days of age) when compared to age-matched, seizure-resistant C57BL/6J mice. The DBA/2J strain had higher benzodiazepine binding both in normal state and in seizures. This higher binding in DBA/2J was due to a higher benzodiazepine receptor density (Bmax) in this strain.

The neurochemical studies demonstrated that brain monoamine deficits could be detected in the projection areas of both NA-containing neuronal groups - locus ceruleus and the lateral tegmental area. These deficits existed in GEPRs without seizure experience and were more pronounced in the GEPR-9s as compared to GEPR-3s. GABA and taurine levels were also abnormal (Laird et al., 1984). It was proposed that the NA and 5-HT deficits were the causes of AE proneness, while GABA-taurine anomalies were "the inadequate attempts of the central nervous system to compensate for the seizure-prone state". This statement was proved not to be the case later (see Lasley, 1991).

GABA concentrations were lower in GEPRs compared to non-epileptic controls in several brain regions. Aspartate and taurine content was also elevated although not in all brain areas investigated (Lasley, 1991). Seizure experience induces the changes in this pattern the increases in aspartate, glutamate and glycine levels took place (as compared to seizurenaive rats) in inferior colliculus and in sensomotor and frontal cortices. Author suggested that the high levels of taurine in GEPR-3s and the elevated content of aspartate in GEPR-9s play certain roles as determinants of seizure severity, as well as low concentrations of GABA did. The seizure-induced changes in brain aspartate and glutamate supported the concept that these excitatory amino acids mediate changes in seizure predisposition (Lasley, 1991, Molnar et al., 2000). In estimating the deviations of brain neurotransmitter levels the main attention was paid to their regional concentrations, inferior colliculi, PAG and substantia nigra being of prime interest. The incomplete seizure suppressant effect of naloxone was moderate, while blockade of NMDA receptors by AP7 or activation of GABA (A) receptors in the PAG really suppressed AE susceptibility. Thus, PAG was viewed as the important link in the chain of structures, necessary for AE fits with GABA (A), opioid peptide and NMDA receptors participating in fit severity regulation (N'Gouemo \& Faingold, 1999). The 1,9-fold increase of brain glutamate, 2,3-fold increase in GABA, 2,4-fold increase of taurine in comparison to non-epileptic rats was found in inferior colliculi by Ribak et al. (1988). In the GEPRs bilateral microinjections of NMDA receptor antagonists in substantia nigra blocked or reduced the seizure severity. The technique of brain micro dialysis permitted to measure the reactivity of respective neurotransmitter systems in GEPRs and non-epileptic rats. It was demonstrated that the increases (relative to basal levels) for non-epileptic controls were $35 \%, 74 \%, 68 \%, 847 \%$ and $283 \%$ for aspartate, glutamate, glycin, taurine and GABA respectively. Corresponding increases for GEPR-9s were less intense - 14\%, 10\%, $41 \%, 505 \%$ and $123 \%$ (Doretto et al., 1994).

Akbar et al. (1998) demonstrated differences in the expression levels of mRNA for three amino acid transporters - glial and neuronal glutamate transporters (GLT-1 and EAAC-1), and the neuronal GABA transporter (GAT-1). Reductions in GAT-1 mRNA were found in genetically epileptic-prone rats in all brain regions examined. Similar reductions in GLT-1 mRNA expression levels were seen in cortex, striatum, and hippocampal CA1 of genetically 
epileptic-prone rats; the largest reduction being in the inferior colliculus. Differences in messenger RNA levels for GLT-1 and EAAC-1 were not reflected or were reflected only partially in the expression of the corresponding proteins (Akbar et al., 1998).

The data obtained for KM strain were more or less similar (Raevski et al., 1995, Kosacheva et al., 1998). The baseline content of serotonin and its metabolite (5-oxyindoleacetic acid) in the temporal cortex, hippocampus and medulla as well as the striatal content of dopamine and 3, 4-dihydroxyphenilacetic acid were higher in KMs than in non-epileptic Wistars. The noradrenalin level in striatum was reduced. Audiogenic seizure fit development in KM rats induced the decrease of GABA level in: striatum, hippocampus and temporal cortex, as well as the reduction of striatal and hippocampal glutamate levels (fig.3). In KM rats the histamine levels in the striatum, hippocampus, amygdala, midbrain, thalamus and hypothalamus were significantly lower than in the epilepsy-resistant Wistar rats (Onodera et al., 1992). At the early stage of KM rats selection the caffeine infusions were the experimental technique to increase the audiogenic fit severity (Krushinsky, 1964). Thus the purinergic system could be considered as being involved into generation of the AE fit.

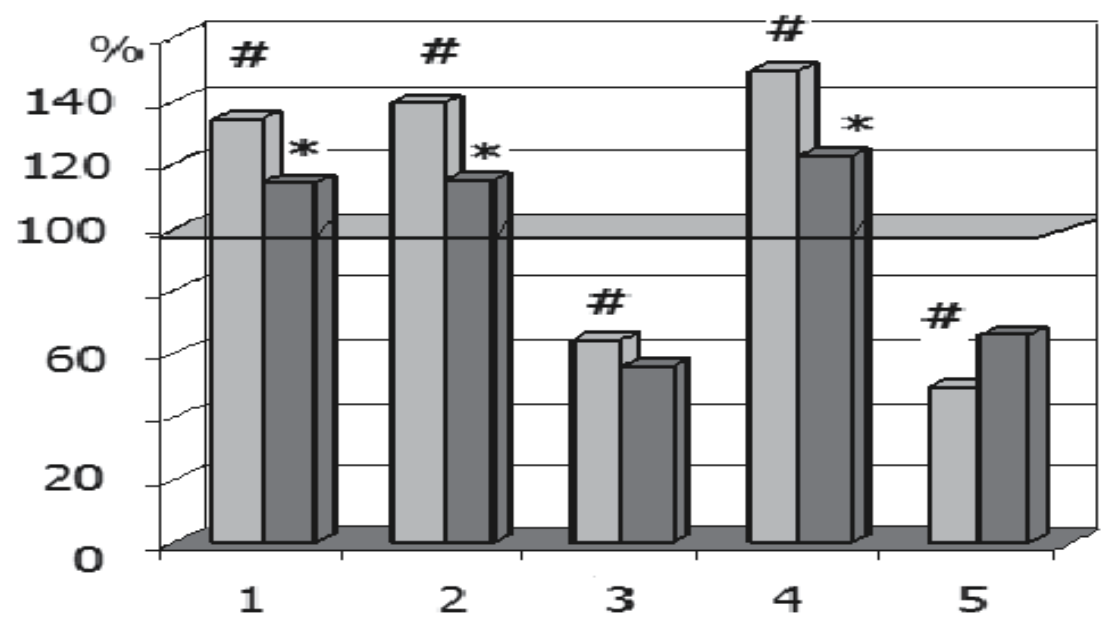

Fig. 3. Medulla levels of excitatory aminoacids (KM rat strain) without seizures (light gray columns) and at the maximum of tonic convulsions in response to sound (dark grey columns) (Raevski et al., 1995). The data presented as the percentage from intact Wistar rats respective level (100\%). 1- aspartate, 2-glutamate, 3-GABA, 4-glycine, 5 - taurine. \# significantly different from Wistar rats, $\mathrm{p}<0.05,{ }^{*}$ - significantly different from the preseizure level, $\mathrm{p}<0.05$

Brain dopamine system also participates in the complicated pattern of monoamine "audiogenic" pattern (Yu et al., 2000, Sorokin et al., 2004). The combined action of dopaminergic and serotonergic agonists suppressed audiogenic seizures, with local increase in dopamine and reduction in serotonin in the striatum (in rats not selected for AE). The audiogenic seizures which were provoked in rats by the withdrawal from chronic ethanol consumption were associated with increase in striatal dopamine and a reduction in striatal serotonin (Yu et al., 2000). In KM rats and the basal striatal dopamine level measured by in vivo microdialysis was $25 \%$ higher that in non-epileptic Wistars. A single amphetamine injection (1 mg/kg body weight, intraperitoneously) caused a significant increase in the 
dopamine basal level up to $250-260 \%$ in animals of both genotypes. However, in Wistar rats, the dopamine level reached maximum as soon as 20 min after drug infusion, whereas in KM rats, this happened after $120 \mathrm{~min}$. The increase in the dopamine level after a single injection of raclopride (antagonist of D2 and D3 receptors) was also similar in amplitude in rats of both genotypes (up to about 210\%); however, this occurred 20-30 and $100 \mathrm{~min}$ after raclopride administration in Wistar and KM rats, respectively. This evidence confirmed that the genetic "defect" in audiogenic seizure prone KM rats is connected with brain monoamines anomalies. At the same time the peculiar scheduling of changes in dopamine increase could be connected with regulatory gene(s) dysfunction (Sorokin et al., 2004).

It is now recognized that neuronal hyperexcitability and excessive production of free radicals have been implicated in the pathogenesis of epilepsy. The high rate of oxidative metabolism, coupled with the low antioxidant defenses and the richness in polyunsaturated fatty acids, makes the brain highly vulnerable to free radical damage (Devi et al., 2008). Prolonged seizures may result in the mitochondrial dysfunction and increased production of reactive oxygen species and nitric oxide, which precede neuronal cell death and cause subsequent epileptogenesis. Emerging evidences also showed that intrinsic mitochondrial apoptotic pathway may contribute to the neuropathology of human epilepsy (Chuang, 2010).

In $\mathrm{KM}$ rats the background levels of the some membrane lipid fractions (containing endogenous antioxidants) were higher in comparison to non-epileptic Wistars. The development of audiogenic seizure fit was accompanied by the significant increase in free radicals levels and lipid peroxides while the lipid antioxidative activity decreased. These changes were found in medulla, thalamus and hippocampus but not in the neocortex (Fedotova et al., 1988). The decrease of brain antioxidant levels developed in parallel with the audiogenic fit stages. Thus it was concluded that these oxidative stress mechanism which participated in these processes also play the important role in the brain reaction to sound induced seizures.

The in vitro studies (incubation of brain synaptosomes and mitochondria of KM rats with lipid peroxidation inducers) demonstrated the decrease of deamination of serotonin (which is the substrate of MAO-A) in mitochondria, but not in synaptosomes with simultaneous stimulation of GABA deamination process. The latter was apparently due to modification of catalytic properties of brain membrane-bound MAO (Medvedev et al., 1992). The similar parameters of KM brain taken at the height of AE seizures demonstrated the stimulation in brain synaptosomes and mitochondria of lipid peroxidation. This was accompanied by a marked decrease in serotonin deamination, with a simultaneous increase in GABA deamination in both fractions. The data obtained suggested that appearance of GABAdeaminating activity might be the important component in the development of epileptic seizures and it was shown that this deamination was determined by the modification of catalytic properties of MAO (Medvedev et al., 1992).

In DBA/2J mice the combined inhibition of serotonin uptake and oxidative deamination attenuated audiogenic seizures. The administration of MAO-A inhibitor (clorgyline) suppressed these AE seizures and the inhibition of seizures by tryptophan was potentiated by combination with either of the mixed MAO inhibitors (Sparks, Buckholtz, 1985).

Brain cytokine system (interleukins $-1,-2,-6$ ) also participate the seizure development and demonstrate differences in mice of different genotypes. In Frings and DBA/2J mice the expression of interleukine-1-alpha transcripts in different brain regions after the AE seizures was elevated in hypothalamus but not in hippocampus presumably by dexamethasonesensitive pathway (Gahring et al., 1997). Intracerebroventricular administration of 
interleukin-2 (both recombinant human and mouse varieties) in DBA/2 mice, increased the incidence of seizures. Since interleukin-2 proconvulsant properties were antagonized by specific monoclonal antibodies, it was suggested that some epileptic phenomena could be linked to stimulation of IL-2 receptors (De Saro et al., 1994). Deficiency in interleukin-6 (IL-6 -/- KO) induce numerous changes in brain metabolism, namely in glutamate, aspartate, GABA, glycine and taurine in different brain structures and these mice developed the audiogenic seizure fits, although no differences between IL-6 WT and KO mice in the susceptibility to maximal electroshock were noted (De Luca et al., 2004).

As mentioned above, brain monoamines levels and metabolism were consistently reported to be different from non-epileptic animals in cases of rodent audiogenic seizures. The biological degradation of monoamines is catalyzed by monoamine oxidase. Tribulin is a fraction of endogenous MAO inhibitors which are detected in human and animal tissues and biological fluids. An investigation of the biological properties of tribulin revealed its heterogeneity and some chemical components were identified. The development of several brain pathologies (epilepsy among them) are accompanied by qualitative changes in catalytic ability of the membrane-bound MAO-A and MAO-B (Medvedev, 1999). Brain tribulin activity in $\mathrm{KM}$ rats was studied after audiogenic seizures of different intensity. Moderate and especially maximal audiogenic seizures were accompanied by the increase of both MAO A inhibitory activity (up to 2.5-fold in case of tonic seizures), this increase in sound exposed nonepileptic Wistars was significantly less intense (Medvedev et al., 1991).

Investigation of phosphorylation in homogenates of neocortex and hippocampus, aiming to discover $\mathrm{AE}$ proneness influence on $\mathrm{Ca}(2+)$ /calmodulin- and $\mathrm{CAMP}$-dependent systems was performed. Non-epilectic Wistar rats, not-pure bred KM audiogenic seizure prone rats and the same rats after being exposed to audiogenic kindling were the subjects of this study. The significant differences in phosphorylation of 270,58, 54 and $42 \mathrm{kDa}$ proteins in neocortex and hippocampus were found. The activity of PKA was higher both in neocortex and hippocampus in audiogenic sensitive rats. Daily repeated audiogenic seizures induced the decrease of $\mathrm{Ca}(2+)$-independent CAMKII activity in hippocampus and the increase of PKA activity in non-epileptic rat neocortex in comparison to audiogenic rats (Yechikhov et al., 2001). Thus the neurochemical studies demonstrated both the similarity and differences in the shifts of main neurotransmission systems in rats and mice, susceptible to AE. The current problem seems to be to detect which brain chemistry and/or gene expression peculiarities are the primary ones (more close to initial genetic defect) and which - develop as the consequences of primary defects. The high complexity of neuronal signaling pathways permit such suggestion, and could tune researchers to the pessimistic mode. Although the detailed knowledge of gene expression during brain development which is extensively investigated at present could bring the quick and may be unexpected data in this respect.

\subsection{Krushinsky's views on AE phenotype: Pure historical interest or the insight with present scientific significance}

At the start of audiogenic epilepsy studies (at the beginning of 1950s) L.V. Krushinsky created the hypothesis that this pathology could be explained by the misbalance between brain excitation and inhibition processes. We should remember that Moruzzi and Magoun first published their data on reticular activation system only in 1949, and there were no ways to discuss in the USSR the possible impact of genotype in AE development, although KM data were obtained via the genetic selection. According to L. Krushinsky's hypothesis the following sequence of events took place in AE rat CNS after the sound onset. Strong 
sound induces the excitation which "irradiates" along the brain stem structures and wild run stage starts. In response to this start the inhibitory process initiates which could be regarded as the reaction against anomalous excitation. As the result the wild run stage could stop. Then there could be two options of events development: i) the fit stops and no further abnormal reactions take place (that will be the audiogenic fit of low intensity, Krushinsky's arbitrary unit "1"), or ii) there occurs only the "inhibitory pause" in the wild run behavior lasting several seconds. As this pause ends the wild run renews and in majority of cases the motor seizure occurs (arbitrary units 2-4). According to Krushinsky's view the prolongation of sound action during the inhibitory pause leads to the "exhaustion" of inhibition as it is too strong. He hypothesized that the inhibitory pause which inserts into the wild run stage (creating "two waves" of wild locomotion) is the "active" inhibitory process. The finish of motor seizures after the "second wave" of the wild run and clonic-tonic convulsions is the result of "over-limit" inhibition (that is of inhibition which develops as the response to very strong stimulation). Bromide infusions accentuated the "inhibitory pause" in the audiogenic fit sequence, and induce such pause in rats which previously showed the "single wave" pattern of wild run. Caffeine's effect was the reverse - the "inhibitory pause" of wild run dissappeared and the fit intensity increased.

At present some arguments in the favor of that old theoretical consideration could be found, that is arguments for benefit of the postulated brain macro-processes and their interactions. One of them is the well known fact concerning the lack of epileptic EEG discharges in the neocortex and hippocampus during the $\mathrm{AE}$ fit. It could be partly ascribed to the "inhibited state" of these structures. Now it was demonstrated that audiogenic fit development is accompanied by the neocortical spreading depression (Vinogradova et al., 2005), which also inhibit both EEG and single unit activity in this structure. The anomalies in the balance of excitatory and inhibitory aminoacid transmitters which are inherent to AE and which are accentuated during and after the $\mathrm{AE}$ fit also could be the manifestation of these two processes interactions. The data also exist which concern the induction of c-Fos expression in neocortex as the result of audiogenic seizure fit, which means that the excitation of GABA-ergic interneurons could explain this finding (e.g. Batuev et al., 1997).

\section{Conclusion}

Many data obtained evidence that the AE susceptibility is associated with the decreased thresholds for evoking seizures of other types (e.g. Scarlatelli-Lima et al., 2003). In WARs the general seizure susceptibility was elevated in comparison to non-epileptic Wistar rats in experiments using other pro-convulsive stimuli (apart from loud sound) - transauricular electroshock, pentylenetetrazole and pilocarpine).

The similar patterns of relationships were found in mouse studies. Audiogenic seizures in mice selectively bred for susceptibility and resistance to ethanol withdrawal (induction of seizures during handling) were investigated. Resistant mice exhibited no AE response at any age, whereas "seizure withdrawal prone" mice were sensitive on several age (days 17, 22, and 28). These data suggested that susceptibility to $\mathrm{AE}$ and handling-induced convulsions during $\mathrm{EtOH}$ withdrawal may share some common genetic determinants and presumably some common neurochemical systems. This phenomenon could be regarded as the AE susceptibility induced by changes in brain neurochemistry induced by ethanol consumption. Authors suggest that these two types of seizures may share some common genetic determinants and presumably some common neurochemical systems (Feller et al., 1994). 
The AE fit pattern in specialized genetic strains of rats and mice is more or less similar and this fact gives the possibility to suggest the existence of the specific "audiogenic epilepsy endophenotype". Gould, Gottesman (2006) introduced the endophenotype notion and described the endophenotypes as "quantifiable components in the genes-to-behaviors pathways". The endophenotype concept has emerged as a strategic tool in neuropsychiatric research, but it has more general applicability. Endophenotypes, as Gould \& Gottesman (2006) stated, can be neurophysiological, biochemical, endocrine, neuroanatomical, cognitive or neuropsychological. Such pattern of specific features could be regarded as complex but rather specific trait of given phenomenon. It is evident that the complex relationships between genes and behavior could not be exactly reproduced in different experiments with different objects (namely in different AE models). Although the analysis of whole plethora of AE data obtained from this point of view - that there is hypothetical "common pathway" by which this pathology could be realized in the brain - could help in understanding of this phenomenon. The endophenotype for audiogenic epilepsy could be the specific seizureprovoking constellation of genetic and neurochemical events, which act both at the level of peripheral hearing organ and at the level of brain structures. The development of regional specific channellopathies and/or of the misbalance in GABA and glutamate systems (both central and cochlear) could be the possible links which help in the search of the endogphenotype of interest. The key components of any useful endophenotype are heritability and stability (state independence). Endophenotype approach is useful as "it reduces the complexity of symptoms and multifaceted behaviors" (Gould \& Gottesman, 2006) which result in identifying units of analysis that could be explored in animal models.

The data reviewed above demonstrated that apart from phenotypical similarity of $\mathrm{AE}$ seizures there are not many traits common for all models explored - these are the disturbances of hearing system and the misbalance of brain glutamate and GABA-ergic system. The malfunction of other traits - brain monoamine, purinergic, histaminergic, cytokine systems dysfunction were also described, although the pattern of changes in cases of $\mathrm{AE}$ were not always consistent in different models and vary with different techniques used, animal models and species chosen (rat-mouse).

It looks more or less plausible that the disturbances in gene expression patterns rather early in development could underlie the $\mathrm{AE}$, although these disturbances (mutations) should be still compatible with the fetus survival and future animal viability (although probably reduced). Such mutations are still to be identified, although the examples of genetic elements which could belong to such category are now available.

The simultaneous genetic inactivation of three transcriptional factors connected with circadian rhythmicity (albumin D-site-binding protein proline, hepatic leukemia factor and thyrotrophic embryonic factor) induce the audiogenic epilepsy in young mice (Gachon et al., 2004). The target gene of this transcription factors family is the pyridoxal kinase $(P d x k)$, which catalyses the conversion of vitamin B12 derivatives into pyridoxal-phosphate. The latter is the coenzyme of many enzymes participating in the neurotransmitter metabolism. It was demonstrated that in the triple mouse KOs the levels of brain pyridoxal-phosphate, serotonin and dopamine were decreased (Gachon et al., 2004).

Mutations of other genes which are identified as being involved in the early stages of CNS development could also be the possible candidates for causes of AE development. One example of such mutation could be the disheveled genes. The proteins coded by this gene family are the important signaling components of beta-catenin/Wnt pathway, which controls cell proliferation and patterning, and the planar cell polarity pathway. Dvl3 (-/-) 
mice died perinatally with several developmental defects, the misorientated stereocilia in the organ of Corti being among them (Etheridge et al., 2008).

The specific pattern of the hypothesized abnormal "developmental" genes expression could be the cause of the CNS disturbances and of the AE in particular. These anomalies presumably could induce the region specific channelopathies in the structures involved in sound-seizure production. As different channellopathies (not yet well understood) are the prominent trait of many human types of epilepsy, the study of genetic/physiological basis of their regional specificity (in AE models) could be of certain clinical value. Although another hypothesis could be tested as well, that such region-specific channelopaties arise in rats and mice as the result of the abnormal pattern of CNS development per se, the rodent brain being especially vulnerable for loud sound as these animals rely on sound sensitivity in avoiding danger in natural habitats.

\section{Acknowledgement}

Partly supported by RFBF (grants NN 04-04-48445, 07-04-00287, N 09-04-0048)

\section{References}

Akbar M.T., Rattray M., Williams R.J., Chong N.W., Meldrum B.S. (1998). Reduction of GABA and glutamate transporter messenger RNAs in the severe-seizure genetically epilepsy-prone rat. Neurosci., Vol. 85, No.4, pp. 1235-1251.

Bagri A., Tomaz C., Brandao M.L., Carrive P. (1989). Increased susceptibility of detelencephalated rats to audiogenic seizures induced by microinjection of bicuculline into the inferior colliculus. Braz. J. Med. Biol. Res., Vol.22, pp. 1361-1370

Bagri A., Sandner G., Di Scala G. (1991). Wild running and switchoff behavior elicited by electrical stimulation of the inferior colliculus: effect of anticonvulsant drugs. Pharmacol. Biochem. Behav., Vol.39, pp. 683-688

Bakhit C., Shenoy A.K., Swinyard E.A., Gibb J.W. (1982). Altered neurochemical parameters in the brains of mice (Frings) susceptible to audiogenic seizures. Brain Res., Vol.244, No.1, pp. $45-52$

Banko M.L., Allen K.M., Dolina S., Neumann P.E., Seyfried T.N. (1997). Genomic imprinting and audiogenic seizures in mice. Behav. Genet., Vol.27, No.5, pp. 465-475

Batuev A.S., Bragina T.A., Aleksandrov A.S., Riabinskaia E.A. (1997) Audiogenic epilepsy: a morphofunctional analysis. Zh. Vyssh. Nerv. Deiat.,Vol.47, No.2, pp. 431-438 (rus)

Becker AJ, Pitsch J, Sochivko D, Opitz T, Staniek M, Chen CC, Campbell KP, Schoch S, Yaari Y, Beck H. (2008). Transcriptional upregulation of Cav3.2 mediates epileptogenesis in the pilocarpine model of epilepsy. J. Neurosc., Vol.28, No.49, pp. 13341-13353

Brennan T.J., Seeley W.W., Kilgard M., Schreiner C.E., Tecott L.H. (1997). Sound-induced seizures in serotonin 5-HT2c receptor mutant mice. Nat Genet.,Vol.16, No.4, pp.387390

Booker J.G., Dailey J.W., Jobe P.C., Lane J.D. (1986). Cerebral cortical GABA and benzodiazepine binding sites in genetically seizure prone rats. Life Sci., Vol.39, No.9, (Sep 1), pp. 799-806

Browning R.A., Wang C., Nelson D.K., Jobe P.C. (1999). Effect of precollicular transection on audiogenic seizures in genetically epilepsy-prone rats. Exp Neurol., Vol.155, No.2, pp. 295-301 
Camarero G., Avendan C., Fernandez-Moreno C., VillarA., Contreras J., lora de PabloF,. Pichel J.G, Varela-Nieto I. (2001). Delayed inner ear maturation and neuronal loss in postnatal Igf-1-deficient mice. J. Neurosc., Vol.21, No.19, pp. 7630-7641

Chakravarty D.N. \& Faingold C.L. (1999). Differential roles in the neuronal network for audiogenic seizures are observed among the inferior colliculus subnuclei and the amygdala. Exp Neurol., Vol.157, No.1, (May) pp. 135-141

Chen C. S. \& Fuller J.L. (1976). Selection for spontaneous or priming-induced audiogenic seizure susceptibility in mice. J. Comp. Physiol. Psychol., Vol.90, No.8, pp. 765-772

Chen L. \& Toth M. (2001). Fragile X mice develop sensory hyperreactivity to auditory stimuli. Neurosci., 2001, v.103, No. 4, pp. 1043-1050

Christopher K. E. \& Smith D.W. (2003). Protection from acoustic trauma is not a primary function of the medial olivocochlear efferent system. J. Assoc. Res. Otolaryngol., Vol.4, No.4, (Dec), pp. 445-465

Chuang Y.C. (2010). Mitochondrial dysfunction and oxidative stress in seizure-induced neuronal cell death. Acta Neurol Taiwan, Vol.19, No.1, pp. 3-15

Clemmesen L., Ingvar M., Hemmingsen R., Bolwig T.G. (1988). Local cerebral glucose consumption during ethanol withdrawal in the rat: effects of single and multiple episodes and previous convulsive seizures. Brain Res., Vol.453, No.1-2, (Jun 21), 204-214

Clough R.W., Eells J.B., Browning R.A., Jobe P.C. (1997). Seizures and proto-oncogene expression of fos in the brain of adult genetically epilepsy-prone rats. Exp Neurol., Vol.146, No.2, (Aug), pp. 341-353

Consroe P., Picchioni A., Chin L. (1979). Audiogenic seizure susceptible rats. Fed Proc., Vol.38, No10, pp. 2411-2416.

Dailey J.W., Yan Q.S., Adams-Curtis L.E., Ryu J.R., Ko K.H., Mishra P.K., Jobe P.C. (1996). Neurochemical correlates of antiepileptic drugs in the genetically epilepsy-prone rat (GEPR). Life Sci., Vol.58, No.4, pp. 259-266

Dallos P. (1992). The active cochlea. J. Neurosci., Vol.2, No.12, pp.4575-4585

De Luca G., Di Giorgio R.M., Macaione S., Calpona P.R., Costantino S., Di Paola E.D., De Sarro A., Ciliberto G., De Sarro G. (2004). Susceptibility to audiogenic seizure and neurotransmitter amino acid levels in different brain areas of IL-6-deficient mice. Pharmac., Biochem. Behav., Vol. 78, No.1-2, (May), pp. 75-81

Depaulis A., Marescaux C., Liu Z., Vergnes M. (1990). The GABAergic nigro-collicular pathway is not involved in the inhibitory control of audiogenic seizures in the rat. Neurosci Lett.,Vol.111, No.3, (Apr 6), pp. 269-274

Deransart C., Le-Pham B.T., Hirsch E., Marescaux C., Depaulis A. (2001). Inhibition of the substantia nigra suppresses absences and clonic seizures in audiogenic rats, but not tonic seizures: evidence for seizure specificity of the nigral control. Neurosci., 36, Vol.105, No.1, pp.203-211

Devi P.U., Manocha A., Vohora D. (2008). Seizures, antiepileptics, antioxidants and oxidative stress: an insight for researchers. Expert Opin. Pharmacother., Vol.9, No.18, (Dec), pp. 3169-3177

De Sarro G., Rotiroti D., Audino M.G., Gratteri S., Nisticó G. (1994). Effects of interleukin-2 on various models of experimental epilepsy in DBA/2 mice. Neuroimmunomod., Vol.1, No.6, (Nov-Dec), pp. 361-369 
Doretto M.C., Burger R.L., Mishra P.K., Garcia-Cairasco N., Dailey J.W., Jobe P.C. (1994). A microdialysis study of amino acid concentrations in the extracellular fluid of the substantia nigra of freely behaving GEPR-9s: relationship to seizure predisposition. Epilepsy Res., Vol.17, No.2, (Feb), pp. 157-165

Doretto M.C., Fonseca C.G., Lobo R.B., Terra V.C., Oliveira J.A., Garcia-Cairasco N. (2003). Quantitative study of the response to genetic selection of the Wistar audiogenic rat strain (WAR). Behav Genet., Vol.33, No.1, (Jan), pp. 33-42.

Doretto M.C., Cortes-de-Oliveira J.A., Rossetti F., Garcia-Cairasco N. (2009). Role of the superior colliculus in the expression of acute and kindled audiogenic seizures in Wistar audiogenic rats. Epilepsia, Vol.50, No.12, (Dec), pp. 2563-2574

Dutra Moraes M.F.D., Galvis-Alonso O.Y., Garcia-Cairasco N. (2000). Audiogenic kindling in the Wistar rat: a potential model for recruitment of limbic structures. Epilepsy Res., Vol.39, No.3, (May), 251-259

Eells J.B., Clough R.W., Browning R.A., Jobe P.C. (2004). Comparative fos immunoreactivity in the brain after forebrain, brainstem, or combined seizures induced by electroshock, pentylenetetrazol, focally induced and audiogenic seizures in rats. Neurosc., Vol.123, No.1, pp. 279-292

Etheridge S.L., Ray S., Li S., Hamblet N.S., Lijam N., Tsang M., Greer J., Kardos N., Wang J., Sussman D.J., Chen P., Wynshaw-Boris A. (2008). Murine dishevelled 3 functions in redundant pathways with dishevelled 1 and 2 in normal cardiac outflow tract, cochlea, and neural tube development. PLoS Genet., Vol.4, No.11, e1000259

Fadiukova O.E., Kuzenkov V.S., Reutov V.P., Krushinskiǔ A.L., Buravkov S.V., Koshelev V.B. (2005). Antistress and angioprotective influence of nitric oxide in epilepsyprone rats of Krushinskiǔ-Molodkina strain. Ross. Fiziol. Zh. Im I.M. Sechenova, Vol.91, No.1, pp.89-96 (rus).

Faingold C.L., Walsh E.J., Maxwell J.K., Randall M.E. (1990). Audiogenic seizure severity and hearing deficits in the genetically epilepsy-prone rat. Exp Neurol., Vol.108, No.1, pp.55-60

Faingold C.L. (1999). Neuronal networks in the genetically epilepsy-prone rat. Adv Neurol.; Vol.79, pp.311-321

Fedotova I.B., Surina (Sourina) N.M., Malikova L.A., Raevskiŭ K.S., Poletaeva I.I. (2008). The investigation of cataleptic muscle tonus changes in rats after audiogenic seizures. Zh. Vyssh. Nerv. Deiat.. Vol.58, No.5, pp.620-627 (rus)

Fedotova I.B., Semiokhina A.F., Arkhipova G.V., Nikashin A.V., Burlakova E.B. (1988). Differences in the lipid composition of the brain of rats of the Krushinski1Molodkina strain during an audiogenic seizure attack and in myoclonus. $\mathrm{Zh}$. Vyssh. Nerv. Deiat., Vol.38, No.2, pp.374-377 (rus).

Fedotova I.B., Semiokhina A.F., Kosacheva E.S., Bashkatova V.G., Raevskiŭ K.S. (1996). The effect of lamotrigine and carbamazepine on the development of the audiogenic seizure reaction in rats of the Krushinskiur-Molodkina strain. Eksp Klin Farmakol., Vol.59, No.6, pp.6-9 (rus)

Fedotova I.B., Kostyna Z.A., Poletaeva I.I., Kolpakov V.G., Barykina N.1 N., Aksenovich T.I. (2005). Genetic analysis of the predisposition to audiogenic seizure fits in Krushinsky-Molodkina rat strain. Russ. J. Genetics, Vol.41, No.11, pp.1487-1494 
Fehr C., Shirley R.L., Metten P., Kosobud A.E., Belknap J.K., Crabbe J.C., Buck K.J. (2004). Potential pleiotropic effects of Mpdz on vulnerability to seizures. Genes Brain Behav., Vol.3, No.1, pp.8-19

Feller D.J., Bassir J.M., Crabbe J.C., Le Fevre C.A. (1994). Audiogenic seizure susceptibility in WSP and WSR mice. Epilepsia, Vol.35, No.4, pp.861-867

Feng H.J., Naritoku D.K., Randall M.E., Faingold C.L. (2001). Modulation of audiogenically kindled seizures by gamma-aminobutyric acid-related mechanisms in the amygdala. Exp. Neurol., Vol.172, No.2,pp. 477-481

Ferraro T.N., Smith G.G., Schwebel C.L., Lohoff F.W., Furlong P., Berrettini W.H., Buono R.J. (2007). Quantitative trait locus for seizure susceptibility on mouse chromosome 5 confirmed with reciprocal congenic strains. Physiol. Genomics., Vol.31, No.3,pp. 458462.

Fless D.A., Salimov R.M. (1974). An analysis of the phase of prespasmodic motor excitation in rats with audiogenic seizures. Biull. Eksp. Biol. Med., Vol.78, No.9, p.31-33 (rus)

Frings H., Frings M. (1951). Otitis media and audiogenic seizures in mice. Science, Vol.113, No.2946, pp.689-690

Frolenkov G.I. (2006) Regulation of electromotility in the cochlear outer hair cell. J. Physiol., Vol.576, Pt 1,pp. 43-48.

Fuller J.L., Easler C., Smith M.E. (1950). Inheritance of audiogenic seizure susceptibility in the mouse. Genetics, Vol.35, No 6, pp.622-632

Fuller J.L., Smith M.E. (1953). Kinetics of sound induced convulsions in some inbred mouse strains. Am. J. Physiol., Vol.172, No.3, pp.661-670

Fuller J.L. (1975) Independence of inherited susceptibility to spontaneous and printed audiogenic seizures in mice. Behav Genet., Vol.5, No 1, pp.1-8

Gachon F., Fonjallaz P., Damiola F., Gos P., Kodama T., Zakany J., Duboule D., Petit B., Tafti M., Schibler U. (2004). The loss of circadian PAR bZip transcription factors results in epilepsy. Genes Dev., Vol.18, No.12. pp.1397-1412

Gahring L.C., White H.S., Skradski S.L., Carlson N.G., Rogers S.W. (1997). Interleukin1alpha in the brain is induced by audiogenic seizure. Neurobiol. Dis., Vol.3, No.4, pp.263-269

Galvis-Alonso O.Y., Cortes De Oliveira J.A., Garcia-Cairasco N. (2004) Limbic epileptogenicity, cell loss and axonal reorganization induced by audiogenic and amygdala kindling in Wistar audiogenic rats (WAR strain). Neurosc., Vol.125, No.3, pp. 787-802

Garcia-Cairasco N. (2002). A critical review on the participation of inferior colliculus in acoustic-motor and acoustic-limbic networks involved in the expression of acute and kindled audiogenic seizures. Hear. Res., Vol.168, pp. 208-222

Garcia-Cairasco N., Sabbatini R.M.E. (1991). Possible interaction between the inferior colliculus and the substantia nigra in audiogenic seizures in Wistar rats. Physiol. Behav., Vol.50, pp. 421-427

Gitai D.L., Martinelli H.N., Valente V., Pereira M.G., Oliveira J.A., Elias C.F., Bittencourt J.C., Leite J.P., Costa-Neto C.M., Garcia-Cairasco N., Paco-Larson M.L. (2010). Increased expression of GluR2-flip in the hippocampus of the Wistar audiogenic rat strain after acute and kindled seizures. Hippocampus, Vol.20, No.1, pp.125-133

Gould T. D. \& Gottesman I. I. (2006). Psychiatric endophenotypes and the development of valid animal models. Genes, Brain and Behavior, Vol.5, pp. 113-119 
Guillaume D., Grisar T., Vergniolle-Burette M. (1991). Glial contribution to seizure: carbonic anhydrase activity in epileptic mammalian brain. Epilepsia, Vol.32, No.1, (Jan-Feb), pp. 10-15

Hall C.S. (1947). Genetic differences in fatal audiogenic seizures between two inbred strains of house mice. J. Hered., Vol. 38, No.1, pp. 2-6

Henry K.R. (1967). Audiogenic seizure susceptibility induced in C57BL-6J mice by prior auditory exposure. Science, Vol.158, No.803, pp.938-940

Henry K.R. (1985). Cochlear function and audiogenic seizures: developmental covariance in the LP/J mouse. Dev Psychobiol., Vol.18, No.6, (Nov), pp. 461-466

Henry K.R. \& Buzzone R. (1986). Auditory physiology and behavior in RB/1bg, RB/3bg, and their F1 hybrid mice (Mus musculus): influence of genetics, age, and acoustic variables on audiogenic seizure thresholds and cochlear functions. J. Comp. Psychol. Vol.100, No.1, (Mar), pp. 46-51

Henry K.R., McGinn M.D., Berard D.R., Chole R.A. (1981). Effects of neonatal thyroxine, genotype, and noise on the ear and audiogenic seizures. J. Comp. Physiol. Psychol., Vol.95, No.3,pp. 418-424

Heron S.E., Scheffer I.E., Berkovic S.F. Dibbens L.M., Mulley J.C. (2007). Channelopathies in Idiopathic Epilepsy. J. Amer. Soc. Exp. NeuroTher.. Vol.4. pp. 295-304

Ivashev M.N., Medvedev O.S., Koshelev V.B., Riasina T.V. (1991). Systemic and regional hemodynamics during audiogenic convulsions in rats genetically epilepsy-prone. Biull Eksp Biol Med. Vol.112, No.12, (Dec), pp. 604-605 (rus)

Jazrawi S.P. \& Horton R.W. (1989). 5-HT2 receptor binding and 5-HT uptake in mouse brain: developmental changes and the relationship to audiogenic seizure susceptibility in DBA/2J mice. Brain Res Dev Brain Res., Vol.45, No.2, (Feb 1), pp. 257-263

Jobe P.C. Relationship of Brain Metabolism to audiogenic seizure in the rat. (1970). Doct. Diss. Tucson, Univ. of Arizona, (cited by Consroe et al., 1979)

Jobe P.C., Picchioni A.L., Chin L. (1973). Role of brain norepinephrine in audiogenic seizure in the rat. J. Pharmacol. Exp. Ther., Vol.184, No.1, (Jan), 1 -10

Jobe P.C., Dailey J.W., Reigel C.E. (1986). Noradrenergic and serotonergic determinants of seizure susceptibility and severity in genetically epilepsy-prone rats. Life Sci., Vol.39, No.9, (Sep 1), pp. 775-782

Jobe P.C., Mishra P.K., Adams-Curtis L.E., Deoskar V.U., Ko K.H., Browning R.A., Dailey J.W. (1995). The genetically epilepsy-prone rat (GEPR). Ital. J. Neurol. Sci., Vol.16, No.1-2, pp.91-99.

Jobe P.C., Mishra P.K., Browning R.A., Wang C., Adams-Curtis L.E., Ko K.H., Dailey J.W. (1994). Noradrenergic abnormalities in the genetically epilepsy-prone rat. Brain Res Bull., Vol.35, No.5-6, pp.493-504

Joulin V.V. \& Pleskacheva M.G. (1991). GABA and diazepam binding inrats of KrushinskyMolodkina strain. Neurochimia, Vol.10, No.1-2, pp. 10-17( rus)

Klein B.D., Fu Y.H., Ptacek L.J., White H.S. (2005). Auditory deficits associated with the frings mgr1 (mass1) mutation in mice. Dev. Neurosci., Vol. 27, No.5, pp.321-332

Kosacheva E.S., Kudrin V.S., Fedotova I.B., Semiokhina A.F., Raevskir K.S. The effect of carbamazepine on the content of monoamines and their metabolites in the brain structures of rats with audiogenic epilepsy. Eksp Klin Farmakol. 1998, v 61, n 3, p. 25-27 (rus). 
Krushinsky L.V. (1964). The development of animal behavior. Normal and abnormal aspects. Consultants Bureau, N.-Y

Krushinsky L.V. (1962). Study of pathophysiological mechanisms of cerebral haemorrages provoked by reflex epileptic seizures in rats. Epilepsia, No.3, pp. 363-380

Krushinski, L.V. (1963). Etude physiologique des differents types de crises convulsives de l'epilepsie audiogene $\mathrm{du}$ rat. Colloques Internationaux $d u$ CNRS, no. 112, Psychophysiologie, Neuropharmacologie et Biochimie de la Crise Audiogene. Editions du CNRS, Paris, pp. 71-92

Krushinsky L.V., Molodkina L.N., Fless D.A., Dobrokhotova L.P., Steshenko A.P., Semiokhina A.F., Zorina Z.A., Romanova L.G. (1970). The functional state of the brain during sonic stimulation. Physiological effects of noise. Welch B.L., Welch A.S., eds., New York, Plenum, pp. 159-183

Kryzhanovskiǔ G.N. (2002). The general theory of pathophysiological mechanisms of neurological and psychopathological syndromes. Zh. Nevrol. Psikhiatr. S S Korsakova, Vol.102, No.11, pp. 4-13 (rus)

Kurtz B.S., Lehman J., Garlick P., Amberg J., Mishra P.K., Dailey J.W., Weber R., Jobe P.C. (2001). Penetrance and expressivity of genes involved in the development of epilepsy in the genetically epilepsy-prone rat (GEPR). J. Neurogenet., Vol.15, No.3-4, pp. 233-244

Kuzntezova G.D., Petrova E.V., Coenen A.M., Van Luijtelaar E.L. (1996). Generalized absence epilepsy and catalepsy in rats. Physiol Behav., Vol.60, No.4, (Oct), pp. 11651169

Laird H.E. 2nd, Dailey J.W., Jobe P.C. (1984). Neurotransmitter abnormalities in genetically epileptic rodents. Fed Proc. Vol.43, No.10, (Jul), pp. 2505-2509

Lasley S.M. (1991) Roles of neurotransmitter amino acids in seizure severity and experience in the genetically epilepsy-prone rat. Brain Res., v. 560, n 1-2, pp.63-70

Liang D., Seyfried T.N. (2001). Genes differentially expressed in the kindled mouse brain. Brain Res. Mol Brain Res., Vol.96, No.1-2, pp. 94-102.

Lints C.E., Willott J.F., Sze P.Y., Nenja L.H. (1980). Inverse relationship between whole brain monoamine levels and audiogenic seizure susceptibility in mice: failure to replicate. Pharmacol Biochem Behav. Vol.12, No.3, (Mar), pp. 385-358

Liu X. Z., Ouyang X.M., Xia X.J., Zheng J., Pandya A., Li F., Li L.D., Katherine O., Welch K.O., Petit C., Richard J.H., Smith R.J.H., Bradley T., Webb B.T., Yan D., Arnos K. S., Corey D., Dallos P., Nance W. E., Chen Z.Y. (2003). Prestin, a cochlear motor protein, is defective in non-syndromic hearing loss. Hum. Mol. Genet., Vol.12, No.10, pp. 1155-1162

Mahendrasingam S., Beurg M, Fettiplace R., Hackney C.M. (2010). The ultrastructural distribution of prestin in outer hair cells: a post-embedding immunogold investigation of lowfrequency and high-frequency regions of the rat cochlea. Eur. J. Neurosci., Vol.31, pp. 1595-1605

McMillan D.R., White P. (2004). Loss of the transmembrane and cytoplasmic domains of the very large G-protein-coupled receptor-1 (VLGR1 or Mass1) causes audiogenic seizures in mice. Mol. Cell. Neurosci., Vol.26, No.2, (Jun), pp. 322-329

Martin B., Chapouthier G., Motta R. (1992). Analysis of B10.D2 recombinant congenic mouse strains shows that audiogenic and beta-CCM-induced seizures depend on different genetic mechanisms. Epilepsia, Vol.33, No.1, pp. 11-13 
Medvedev A.E. (1999). Monoamine oxidase, tribulin, isatin: basic and applied medical aspects. Vestn. Ross. Akad. Med. Nauk, v.10, pp.45-48 (rus)

Medvedev A.E., Rajgorodskaya D.I., Gorkin V.Z., Fedotova I.B., Semiokhina A.F. (1992). The role of lipid peroxidation in the possible involvement of membrane-bound monoamine oxidases in gamma-aminobutyric acid and glucosamine deamination in rat brain. Focus on chemical pathogenesis of experimental audiogenic epilepsy. Mol. Chem. Neuropathol., Vol.16, No.1-2, (Feb-Apr), pp. 187-201

Medvedev A.E., Gorkin V.Z., Fedotova I.B., Semiokhina A.F., Glover V., Sandler M. (1992). Increase of brain endogenous monoamine oxidase inhibitory activity (tribulin) in experimental audiogenic seizures in rats: evidence for a monoamine oxidase. A inhibiting component of tribulin. Biochem. Pharmacol. Vol.44, No.6, (Sep 25), pp. 1209-1210

Merrill M.A., Clough R.W., Jobe P.C., Browning R.A. (2005). Brainstem seizure severity regulates forebrain seizure expression in the audiogenic kindling model. Epilepsia, Vol.46, No.9, (Sep), pp. 1380-1388

Middlesworth, L.V., Norris, C.H. (1980). Audiogenic seizures and cochlear damage in rats after perinatal antithyroid treatment. Endocrinology, Vol.106, pp. 1686-1690

Midzyanovskaya I.S., Kuznetsova G.D., Vinogradova L.V., Shatskova A.B., Coenen A.M., van Luijtelaar G. (2004). Mixed forms of epilepsy in a subpopulation of WAG/Rij rats. Epilepsy Behav.Vol.5, No5, (Oct), pp. 655-661

Millan M.H., Meldrum B.S., Boersma C.A., Faingold C.L. (1988). Excitant amino acids and audiogenic seizures in the genetically epilepsy prone rat. II. Efferent seizure propagating pathway. Exp. Neurol., Vol.99, pp.687-698

Mills S.A., Savage D.D. (1988) Evidence of hypothyroidism in the genetically epilepsy-prone rat. Epilepsy Res., Vol.2, No.2, pp.102-110

Misawa H., Sherr E.H., Lee D.J., Chetkovich D.M., Tan A., Schreiner C.E., Bredt D.S. (2002). Identification of a Monogenic Locus (jams1) Causing Juvenile Audiogenic Seizures in Mice. J. Neurosci., Vol.22, No.23, p.10088-10093

Molnar L.R., Fleming W.W., Taylor D.A. (2000). Alterations in neuronal gamma aminobutyric acid-a receptor responsiveness in genetic models of seizure susceptibility with different expression patterns. J. Pharm. Experim. Therap., Vol.295, No.3, pp. 1258-1266

Moraes, M.F.D., Del Vecchio, F., Terra, V.C., Garcia-Cairasco, N. (2000). Time evolution of acoustic 'information' processing in the mesencephalon of Wistar rats. Neurosci. Lett., Vol.284, pp. 13-16

Moraes M.F.D., Chavalia M., Mishra P.K., Jobe P.C., Garcia-Cairasco N. (2005). A comprehensive electrographic and behavioral analysis of generalized tonic-clonic seizures of GEPR-9s. Brain Res., Vol.1033, p. 1- 12.

Mulley J.C., Scheffer I.E., Petrou S., Berkovic S.F. (2003). Channelopathies as a genetic cause of epilepsy. Curr Opin Neurol. Vol.16, No2., (Apr), pp. 171-176

Naritoku D.K., Mecozzi L.B., Aiello M.T., Faingold C.L. (1992). Repetition of audiogenic seizures in genetically epilepsy-prone rats induces cortical epileptiform activity and additional seizure behaviors. Exp Neurol., Vol.115, No3., (Mar), 317-324

Nehlig A., Vergnes M., Hirsch E., Boyet S., Koziel V., Marescaux C. (1995). Mapping of cerebral blood flow changes during audiogenic seizures in Wistar rats: effect of kindling. J. Cereb. Blood Flow Metab., Vol.15, No.2, pp.259-269 
Neumann P.E.\& Collins R.L. (1991). Genetic Dissection of Susceptibility to Audiogenic Seizures in Inbred Mice. Proc. Natl. Acad. Sci., Vol.88, pp. 5408-5412

Neumann P.E. \& Collins R.L. (1992). Confirmation of the influence of a chromosome 7 locus on susceptibility to audiogenic seizures. Mamm Genome, Vol.3, No.5, pp. 250-253

Neumann P.E. \& Seyfried T.N. (1990). Mapping of two genes that influence susceptibility to audiogenic seizures in crosses of C57BL/6J and DBA/2J mice. Behav Genet. Vol.20, No.2, (Mar), pp. 307-323

Ng L., Pedraza P.E., Faris J.S., Vennström B., Curran T., Morreale de Escobar G., Forrest D. (2001). Audiogenic seizure susceptibility in thyroid hormone receptor betadeficient mice. Neuroreport, Vol.12, No.11, (Aug 8), pp. 2359-2362

N'Gouemo P. \& Faingold C.L. (1996). Repetitive audiogenic seizures cause an increased acoustic response in inferior colliculus neurons and additional convulsive behaviors in the genetically-epilepsy prone rat. Brain Res. , Vol.710, No.1-2, (Feb 26), pp. $92-96$

N'Gouemo P. \& Faingold, C. L. (1998). Periaqueductal gray neurons exhibit increased responsiveness associated with audiogenic seizures in the genetically epilepsyprone rat. Neuroscience, , v 84,N 2,P 619-625

N'Gouemo P. \& Faingold C.L. (1999). The periaqueductal grey is a critical site in the neuronal network for audiogenic seizures: modulation by GABA(A), NMDA and opioid receptors. Epilepsy Res., Vol.35, No.1, (May), pp. 39-46.

N'Gouemo P. \& Faingold C.L. (1999). The periaqueductal grey is a critical site in the neuronal network for audiogenic seizures: modulation by GABA(A), NMDA and opioid receptors. Epilepsy Res, Vol.35, pp. 39-46

N'Gouemo P. \& Morad M. (2003). Voltage-gated calcium channels in adult rat inferior colliculus neurons. Neurosci., Vol.120, No 3, pp. 815-826

N'Gouemo P., Faingold C.L., Morad M. (2009). Calcium channel dysfunction in inferior colliculus neurons of the genetically epilepsy-prone rat. Neuropharm., Vol.56, No.3, pp. 65-75

N'Gouemo P, Yasuda R., Faingold C.L. (2010). Seizure susceptibility is associated with altered protein expression of voltage-gated calcium channel subunits in inferior colliculus neurons of the genetically epilepsy-prone rat. Brain Res. Vol.1308, (Jan 13), pp. 153-157

Onodera K., Tuomisto L., Tacke U., Airaksinen M. (1992). Strain differences in regional brain histamine levels between genetically epilepsy-prone and resistant rats. Methods Find Exp Clin Pharmacol., Vol.14, No.1, pp.13-16.

Pacey L. K., Heximer S.P., Hampson D.R. (2009). Increased GABAB Receptor-Mediated Signaling Reduces the Susceptibility of Fragile X Knockout Mice to Audiogenic Seizures. Mol. Pharmacol., Vol.76, pp. 18-24

Penny J.E., Brown R.D., Hodges K.B., Kupetz S.A., Glenn D.W., Jobe P.C. (1983). Cochlear morphology of the audiogenic-seizure susceptible (AGS) or genetically epilepsy prone rat (GEPR). Acta Otolaryngol.. Vol.95. No.1-2, pp. 1-12

Pierson M. \& Li D. (1996). Cochlear integrity in rats with experimentally induced audiogenic seizure susceptibility. Hear. Res., Vol.101, No.1-2, (Nov 1), pp. 7-13

Pierson M. \& Snyder-Keller A. (1994). Development of frequency-selective domains in inferior colliculus of normal and neonatally noise-exposed rats. Brain Res., Vol.636, No.1, (Feb 4), pp. 55-67 
Pierson M.G.\& Swann J. (1991). Ontogenetic features of audiogenic seizure susceptibility induced in immature rats by noise. Epilepsia, Vol.32, pp. 1-9

Plotnikof N.A. A neuropharmacological study of escape from audiogenic seizures. In: Psychopharmacologie, neuropharmacology et biochimie de la crise audiogene. 1963, CNRS, Paris, VIII

Poletaeva I.I., Lil'p I.G., Bizikoeva F.Z., Ivanov V.I. (1996). Audiogenic epilepsy in 101/HY mice at different periods of postnatal development. Ontogenez; Vol.27, No.3, (MayJun), pp. 222-231

Raevsky K.S., Bashkatova V.G., Koudrine V.S., Malikova L.A., Vitzkova G.Yu., Semiokhina F.F., Fedotova I.B. (1995). The content of neuromediator aminoacids and the products of lipid peroxidation in the brain of rats predisposed to audiogenic seizures. Neurochimia, Vol.12, No4, pp. 47-55.

Reigel C.E., Jobe P.C., Dailey J.W., Stewart J.J. Responsiveness of genetically epilepsy-prone rats to intracerebroventricular morphine-induced convulsions. Life Sci. 1988, v. 42. n 18, p. 1743-1749.

Ribak C.E., Byun M.Y., Ruiz G.T., Reiffenstein R.J. (1988). Increased levels of amino acid neurotransmitters in the inferior colliculus of the genetically epilepsy-prone rat. Epilepsy Res., Vol.2, No.1, (Jan-Feb), pp. 9-13

Ribak C.E., Khurana V., Lien N.T. (1994). The effect of midbrain collicular knife cuts on audiogenic seizure severity in the genetically epilepsy-prone rat. J. Brain Res., Vol.35, pp. 303-311

Ribak C.E. \& Morin C.L. (1995). The role of the inferior colliculus in a genetic model of audiogenic seizures. Anat. Embryol. (Berl)., Vol.191, No.4, (Apr), pp. 279-295

Ribak C.E., Manio A.L., Navetta M.S., Gall C.M. (1997). In situ hybridization for c-fos mRNA reveals the involvement of the superior colliculus in the propagation of seizure activity in genetically epilepsy-prone rats. Epilepsy Res., Vol.26, No.3, pp.397-406

Robertson H.A. (1980). Audiogenic seizures: increased benzodiazepin receptor binding in a susceptible strain of mice. Eur. J. Pharmacol., Vol.66, No.2-3, pp.249-252

Romanova L.G., Zorina Z.A., Korochkin L.I. (1993). A genetic, physiological, and biochemical investigation of audiogenic seizures in rats. Behav Genet., Vol.23, No.5, (Sep), pp. 483-489

Rossetti F., Rodrigues M., Cortes de Oliveira J.A., Garcia-Cairasco N. (2006). EEG wavelet analyses of the striatum-substantia nigra pars reticulata-superior colliculus circuitry: Audiogenic seizures and anticonvulsant drug administration in Wistar audiogenic rats (War strain). Epilepsy Research, Vol.72, pp. 192-208

Ross K.C. \& Coleman J.R. (1999). Audiogenic seizures in the developmentally primed LongEvans rat. Dev. Psychobiol., v. 34, N 4, p. 303-313

Ross K.S.\& Coleman J.R. (2000). Developmental and genetic audiogenic seizure models: behavior and biological substrates. Neurosci. Biobehav. Rev., Vol.24, No.6, pp. 639653

Ryan A.F., Keithley E.M., Wang Z.X., Schwartz I.R. (1990). Collaterals from lateral and medial olivocochlear efferent neurons innervate different regions of the cochlear nucleus and adjacent brainstem. J Comp Neurol., Vol.300, No.4, (Oct 22), pp. 572-582

Scarlatelli-Lima A.V., Magalhaes L.H., Doretto M.C., Moraes M.F. (2003). Assessment of the seizure susceptibility of Wistar Audiogenic rat to electroshock, pentyleneterazole and pilocarpine. Brain Res., Vol.960, No.1-2, pp.184-189 
Schrott A., Stephan K., Spoendlin H. (1989). Hearing with selective inner hair cell loss. Hear Res. Vol.40, No.3, (Jul), pp. 213-219

Semiokhina A.F. (1969). Cortical-subcortical relationshio during neocortex spreading depression. ZH. Vyssh. Nerv. Deuat., Vol.19, No1, pp. 143-149

Semiokhina A.F., Fedotova I.B., Poletaeva I.I. (2006). Rats of Krushinsky-Molodkina strain: studies of audiogenic epilepsy, vascular pathology, and behavior. Zh Vyssh Nerv Deyat., Vol.56, No3, (May-Jun), pp. 298-316 (rus)

Seyfried T.N., Yu R.K., Glaser G.H. (1980). Genetic analysis of audiogenic seizure susceptibility in C57BL/6J X DBA/2J recombinant inbred strains of mice. Genetics. Vol.94, No.3, (Mar), pp. 701-718

Seyfried T.N., Glaser G.H., Yu R.K. (1984). Genetic analysis of serum thyroxine content and audiogenic seizures in recombinant inbred and congenic strains of mice. Exp. Neurol., Vol.83, No.2, pp 423-428

Simler S., Hirsch E., Danober L., Motte J., Vergnes M., Marescaux C. (1994). C-fos expression after single and kindled audiogenic seizures in Wistar rats. Neurosci Lett., Vol.175, No.1-2, (Jul), pp. 458-462

Skradski S.L., Clark A.M., Jiang H., White H.S., Fu Y.H., Ptácek L.J. (2001). A novel gene causing a mendelian audiogenic mouse epilepsy. Neuron, Vol.31, No.4, (Aug 30), 537-544

Sorokin A.Ia., Kudrin V.S., Klodt P.M., Tuomisto L., Poletaeva I.I., Raevskiĭ K.S. (2004). The interstrain differences in the effects of D-amphetamine and raclopride on dorsal striatum dopaminergic system in KM and Wistar rats (microdialysis study). Genetika (rus). Vol.40, No.6, (Jun), pp. 846-849

Sparks D.L., Buckholtz N.S. (1985). Combined inhibition of serotonin uptake and oxidative deamination attenuates audiogenic seizures in DBA/2J mice. Pharmacol. Biochem. Behav., Vol.23, No.5, pp.753-757

Stanojlović O., Zivanović D., Susić V. (2000). N-Methyl-D-aspartic acid- and metaphitinduced audiogenic seizures in rat model of seizures. Pharmacol. Res., Vol.42, No.3, (Sep), pp. 247-253

Statnick M.A., Maring-Smith M.L., Clough R.W., Wang C., Dailey J.W., Jobe P.C., Browning R.A. (1996). Effect of 5,7-dihydroxytryptamine on audiogenic seizures in genetically epilepsy-prone rats. Life Sci., Vol.59, No.21, pp. 1763-1767

Surina (Sourina) N.M., Kalinina T.C.,Volkova A.V., Malikova L.A., Poletaeva I.I., Raevski K.S., Fedotova I.B. (2011). Anxiety and the predisposition to catalepsy in rats of different genotypes Bull. Exp. Biol. Med., Vol.151, No1

Suzuki H., Katayama K., Takao T., Murakami H., Fukuda M., Kawaguchi T., Kakita A., Takahashi H., Kudoh M., Tanaka R., Shibuki K. (2006). Transcranial imaging of audiogenic epileptic foci in the cortex of DBA/2J mice. Neuroreport, Vol.17, No3, (Feb 27), pp. 267-271

Takao T., Murakami H., Fukuda M., Kawaguchi T., Kakita A., Takahashi H., Kudoh M., Tanaka R., Shibuki K. Transcranial imaging of audiogenic epileptic foci in the cortex of DBA/2J mice. Neuroreport. 2006, v 17, n 3, p. 267-271.

Takenaka M., Amakasu K., Saito K., Suzuki K.A. (2009). Spontaneous mutation of the Wwox gene and audiogenic seizures in rats with lethal dwarfism and epilepsy. Genes Brain Behav., Vol.8, No7, (Oct), pp. 650-660 
Terra V.C., Garcia-Cairasco, N. (1994). NMDA-dependent audiogenic seizures are differentially regulated by inferior colliculus subnuclei. Behav. Brain Res., Vol.62, pp. 29-39

Terra V.C., Garcia-Cairasco N. (1992). Neuroethological evaluation of audiogenic seizures and audiogenic-like seizures induced by microinjection of bicuculline into the inferior colliculus. II. Effects of nigral clobazam microinjections. Behav Brain Res. Vol. 7, No.1, pp. 19-28.

Tupal S. \& Faingold C.L. (2006). Evidence supporting a role of serotonin in modulation of sudden death induced by seizures in DBA/2 mice. Epilepsia, Vol.47, No1, (Jan), pp. 21-26

Urban G.P., Willott J.F. (1979). Response properties of neurons in inferior colliculi of mice made susceptible to audiogenic seizures by acoustic priming. Exp Neurol., Vol.63, No.2, pp.229-243.

Willott J.F. \& Lu S.M. (1980). Midbrain pathways of audiogenic seizures in DBA/2 mice. Exp Neurol., Vol.70, pp. 288-299

Willott J.F., Lu S.M. (1982). Noise-induced hearing loss can alter neural coding and increase excitability in the central nervous system. Science, Vol.216, No.4552, pp.1331- 1334

Willott J.F., Lints C.E., Nenja L.H. (1979). Changes in brain levels of NE and 5-HT are not responsible for attenuation of audiogenic seizures by spinal cordotomy. Pharmacol. Biochem. Behav., Vol.10, No.6, (Jun).,pp. 957-958

Vlasov S.G., Storozhenko I.V., Khak A.M., Koshelev V.B., Riasina T.V., Medvedev O.S. (1991). The cardiovascular reactions during audiogenic seizures in rats genetically predisposed to epilepsy Fiziol Zh SSSR, Vol.77, No.4, (Apr), pp. 52-58 (rus)

Vinogradova L.V., Kuznetsova G.D., Coenen A.M. (2005). Audiogenic seizures associated with a cortical spreading depression wave suppress spike-wave discharges in rats. Physiol Behav., Vol.86, N4, (Nov 15), pp. 554-558.

Yechikhov S., Morenkov E., Chulanova T., Godukhin O., Shchipakina T. (2001). Involvement of cAMP- and $\mathrm{Ca}^{2+} /$ calmodulin-dependent neuronal protein phosphorylation in mechanisms underlying genetic predisposition to audiogenic seizures in rats. Epilepsy Res., Vol.46, No1, (Jul), pp. 15-25

Yasuda S., Ishida N., Higashiyama A., Kato N. (1998). Running fit and generalized tonicclonic seizure are differently controlled by different subtype receptors in the brainstem. Nihon Shinkei Seishin Yakurigaku Zasshi., Vol.18, No.4, (Aug), pp. 123-128 (jap)

Yagi H., Takamura Y., Yoneda T., Konno D., Akagi Y., Yoshida K., Sato M. (2005). Vlgr1 knockout mice show audiogenic seizure susceptibility. J. Neurochem., Vol.92, No1, (Jan), pp. 191-202

Yu L., Fisher H., Wagner G.C. (2000). Monoaminergic changes associated with audiogenic seizures in ethanol-dependent rats. Prog. Neuropsychopharmacol. Biol. Psych., Vol.24, No.8, pp. 1379-1392.

Zivanovic D., Susic V., Stanojlovic O. (1997). APH, an N-methyl-D-aspartate receptor antagonist, blocks the metaphit-induced audiogenic seizures in rats. J. Physiol Paris., Vol.91, No.6, (Dec), pp. 317-323 


\section{Part 4}

Ion and Channels 



\title{
Ionic Imbalance
}

\author{
John Robert Cressman, Christine Drown \\ and Monica Gertz \\ Krasnow Institute for Advanced Studies, \\ George Mason University \\ United States of America
}

\section{Introduction}

Seizures are accompanied by significant alterations in both intra- and extracellular ion concentrations (Hotson, 1973; Kager et al., 2000). These concentration gradients, in concert with the conductances of a variety of ion channels, determine the resting membrane potential, the degree of membrane excitability, and the strength of excitatory and inhibitory synaptic responses. Changes in these concentration gradients have profound effects on the signaling and excitability of cells and can therefore play an important role in seizure generation, maintenance and cessation (Kager et al., 2000).

In this chapter, we describe the network of pumps, channels, and exchangers that make up the ionic regulatory system (IRS). This system runs in a nearly steady state while the neuron is at rest and responds to increased neuronal signaling by reestablishing normal ion distributions. We give a brief description of the basic elements of the IRS and their role in ionic maintenance. The IRS has the ability to redistribute intra- and extracellular ions rapidly, but this system can be overwhelmed by excessive activity, allowing ion concentration to drift towards equilibrium permitting their effects to dominate neuronal dynamics. (Ullah et al., 2009; Ransom et al., 2004) In addition to excessive neuronal firing, the IRS can be compromised through a broad range of alterations to its constituent mechanisms. We give a broad overview of how malfunctions in critical elements of the IRS may influence seizure onset or duration and identify several motifs that may play a role in a wide range of seizure disorders.

We will present a brief review of the known etiologies for a small number of seizure disorders. We discuss the ways in which idiopathic causes of epilepsy such as channelopathies, as well as symptomatic origins such as structural malformations and scarring, interact with the IRS to modulate or even generate seizure activity. In the second part of this section we outline the role of the IRS in experimental seizure models, both acute and chronic. We conclude this section by evaluating experimental seizure models and mathematical models based on the mechanisms that comprise the IRS.

In the final section we discuss the action of common anti epileptic drugs and note whenever possible their proposed mechanism in seizure prevention or cessation. We discuss direct and indirect interactions of these drugs upon the IRS and evaluate potential consequences of these interactions. 


\section{The ionic regulatory system}

\subsection{Components of the ionic regulatory system}

The neuron at rest is subject to continuous leakage of ions across the plasma membrane. It therefore requires a steady flow of energy to maintain ionic concentrations necessary for normal neuronal firing. Ions leak down their electrochemical gradients via a combination of diffusion through the membrane as well as channels whose conductances are relatively insensitive to voltage but highly selective to ionic species. These leak currents determine the resting membrane potential and serve to reestablish the resting membrane potential after perturbations, wiping out the effects of inputs received by the neuron in the distant past. It may even be said that leak currents allow the neuron to "forget" remote inputs. The conductance through other channels depends upon factors such as transmembrane potential, ionic concentrations, chemical ligands, and more sophisticated cellular processes. These channels are involved in neurotransmitter signaling and action potential generation. The electrical state of the neuron is determined by the flow of ions across the plasma membrane. The passage of ions through these channels is due to a competition between their charge flow down the electrical gradient and chemical flow down their concentration gradient. The potential at which these mechanisms are balanced is described by the logarithm of the ionic concentrations for a given ion outside and inside the cell and is known as the Nernst potential.

$$
V=26.6 \ln \left[C_{o} / C_{i}\right]
$$

The further the actual membrane potential is from this Nernst potential, the greater the electrochemical gradient for that species of ion. The amount of charge that flows down this gradient can be approximated by Ohm's law, where resistance equals one over the membrane conductivity, $\sigma$, for the cell and the voltage is the difference between the membrane potential and the reversal potential for the ion. In reality, the conductivity is dependent on the ionic concentrations and is better modeled using the Goldman-HodgkinKatz equation. The approach we outline is a good approximation to the GHK analysis and is far more transparent.

$$
I_{i}=\sigma_{i}\left(V_{m}-V_{i}\right)
$$

At rest, the electric current produced by the leak of sodium, potassium, and chloride are balanced, and the resting potential, aside from osmotic effects and immobile ions, is determined by a conductance-weighted average of their reversal potentials. The net current through the ion channels is described as:

$$
I=\sigma\left(V_{m}-V_{\text {rev }}\right)=\sum_{i} \sigma_{i}\left(V_{m}-V_{i}\right)
$$

We can therefore express the total reversal as:

$$
V_{\text {rev }}=\frac{\sum_{i} \sigma_{i} V_{m}}{\sum_{i} \sigma_{i}}
$$


Similarly, the reversal potential for channels that are permeable to multiple ions, such as the NMDA, AMPA, and GABA channels, is roughly a conductance-weighted average of the reversal potentials for the permeable ions. In any case the flow of ions through a pore is proportional to the difference between the membrane potential and the reversal potential for the ion. This difference is the electrochemical gradient experienced by these ions.

Individual ions are not regulated independently, but rather the pumps, channels, and cotransporters providing steady state concentration gradients often exchange precise ratios of different ionic species therefore connecting their individual dynamics. Therefore, the IRS forms a driven-coupled-dynamic system that can display complex behaviors when forced from normal steady state conditions (Cressman, 2009). These emergent behaviors may play a role in seizure dynamics.

Ultimately the driving force behind all ionic gradients is a set of ATPases embedded in the cellular membrane that use the energy stored in ATP to move chemical species and charges against their electrochemical gradients. There are numerous electrogenic exchangers in the human brain, but a few of these play a disproportionately large role in establishing ionic gradients. The primary source of electrochemical gradients across the plasma membrane of neurons is the $3 / 2$ sodium potassium ATPase. This pump extrudes three sodium ions into the extracellular space in exchange for the uptake of two potassium ions into the intracellular space, moving both elements up their chemical gradient as well as te charge up the electrical gradient. The rate of exchange increases with increasing intracellular sodium and extracellular potassium concentrations, providing a mechanism that compensates for increased ionic drain produced by electrical signaling (Lennie, 2003; Attwell, 2001). It is also responsive to the membrane potential, magnesium concentration, and ATP availability. Pumping rates and ATP production are limited by glucose, oxygen and mitochondrial function, therefore there are multiple constraints upon the compensatory action of the pump. Gradients are further tuned by the action of electroneutral cotransporters that move ions up their concentration gradient at the expense of a second, electrochemically favorable ionic gradient. For instance, chloride gradients are largely established through the cotransport of potassium and chloride ions through the $\mathrm{KCl}$ cotransporters (Payne, 1997; Stein, 2003). The action of this cotransporter allows potassium to exit the cell down its electrochemical gradient while forcing chloride up its electrochemical gradient. Therefore the steady state chloride gradients are directly influenced by the potassium gradients establishing more linkage between ion species.

The neuronal environment is also highly regulated by the activity of glial cells surrounding the neurons. Glial cells help maintain a normal neuronal environment through a range of actions, including reuptake of extracellular glutamate and buffering of extracellular potassium concentrations. Astrocytes posses a high density of inward rectifying potassium channels (Kir). The phenomenon of rectification is produced by polyamines or magnesium ions that block the channel at positive potentials, preventing potassium from flowing down its electrochemical gradient into the extracellular space. As potassium is dumped into the extracellular space, its reversal potential increases towards zero and becomes greater than that of the glial membrane potential, thus leading to an inward flow of potassium (Butt, 2006). Once siphoned into the glial cell, potassium diffuses through the network of glial cells via gap junctions that allow networks of glia to form extensive functional groups. These glial networks can move potassium from areas of high to low extracellular potassium concentration. They may also serve to move ions into the vasculature by interacting with 
specialized endothelial cells of the blood brain barrier (BBB). This buffering may mitigate the local excitatory effects of increased extracellular potassium, but it may also facilitate the spread of potassium over relatively large spatial networks (Somjen, 2004).

In addition to glial buffering, simple ionic diffusion and osmosis will blur ionic gradients in the extracellular space. On the scales of the extracellular space the diffusion constant for water can be assumed constant, however the effective diffusion is limited by the path the ions can take. This effect, or tortuosity, can be greatly influenced by the geometry of the extracellular space. Increased extracellular potassium concentrations leads to potassium uptake by glial cells and the subsequent absorption of water through osmotic action. This influx of water leads to cell swelling that in turn can cause the extracellular space to become more confined resulting in an increased tortuosity (Somjen, 2004; Sykova, 2005).

Finally, the brain needs a continual supply of energy, oxygen, and other building blocks as well as the removal of carbon dioxide and other waste. As in the rest of the body this role is provided by the vasculature, however endothelial cells of the central nervous system and gonads are far less permeable than the in the rest of the body. This increased regulation is provided by the BBB and the cerebral spinal fluid barrier (CSFB). Endothelial cells with tight junctions line the capillaries in grey matter to make the BBB, and similar cells line the blood vessels of the choroids plexus and pial and arachnoid spaces as well as the outer layer of cells of the arachnoid (Somjen, 2004; Zheng et al. 2005). These cells actively move ions, amino acids, glucose and more in and out of the vasculature and allow the passive diffusion of oxygen and carbon dioxide. The endothelial cells of the BBB and their tight junctions are influenced by the endfeet processes of the glial syncytium, interactions that are believed to play an important role in the glial buffering of potassium, as discussed above.

One should not think of the IRS as exerting strict control over ionic composition; rather this set of mechanisms supports certain fixed states towards which the system will evolve. However this system can potentially support multiple fixed points, and limit cycles as well as a range of long lived transient states. This lack of strict control could be viewed as a shortcoming of neuronal systems, but in reality it affords them a great degree of dynamics that can be used for changing neuronal function. For instance, calcium entering the buton after the arrival of an action potential is not immediately expelled, and this makes subsequent action potentials more effective at producing vesicle release (Eccles, 1941, Feng, 1941). This common mechanism of synaptic potentiation is utilized by some cells and has been proposed as the basis for information encoding. Other transient neuronal states, such as cortical up-states, may also make use of transient deviations from ionic balance(Lennie, 2003; Haider, 2006; Ullah et al., 2009).

\subsection{Breakdowns of the IRS}

Seizure disorders may be caused by a number of distinct etiologies. Causes are generally classified either as idiopathic, that is, of unknown but likely genetic origin, or symptomatic, that is, due to a lesion or structural malformation (Shimizu-Okabe et al., 2007). A number of genetic abnormalities have been identified as causing idiopathic epilepsy disorders, and for many of these abnormal protein structure and function have been identified. The genetic culprits tend to encode ion channels and receptors for neurotransmitters (Lu \& Wang, 2009). For epilepsy due to structural changes or defects, the cause of the seizure is due to malformations or scarring that cause far less subtle changes in neuronal function. We will proceed by revisiting the main components of the IRS, discuss which components would 
make the system vulnerable, and present examples of epilepsies that are influenced by these breakdowns. We will finish this section by discussing experimental and computational models of epileptic seizures and discuss how these are predicated on disruptions to the IRS. We will begin by describing some general effects of disturbances to the ionic environment on seizure generation and propagation. We will refer back to these motifs in each subsequent section. They are segregated into discussions of resting membrane potential, excitability and refractory periods, edema, acidosis, and synaptic release and response.

\subsubsection{Resting potential}

The magnitude of the resting membrane potential is approximated as the weighted average of the reversal potentials for the ions conducted at rest as described in the previous section. Reversal potentials decrease in magnitude with decreasing concentration gradients, and so decreases in potassium and/or chloride gradients will result in an upward shift of the resting membrane potential and can lead to increased excitability and spontaneous spike generation. On the other hand, decreases in sodium gradients have the reverse effect, making the cell less excitable. The membrane potential is more sensitive to absolute changes in potassium concentrations than absolute sodium concentrations, since potassium is more conductive than sodium. Therefore potassium is the most important ion for establishing the resting membrane potential (Kager et al., 2000).

The potassium reversal potential is also more sensitive to changes in potassium concentrations than are reversal potentials for sodium and chloride. Since reversal potentials depend on the ratio of the extra- and intracellular concentrations an absolute change to the smaller of the two concentration has a greater effect on the reversal potential than would the same change in the larger concentration. Ionic flow across the membrane also produces a larger concentration change in the exterior than in the substantially larger interior space. Since potassium concentrations are lowest in the extracellular space and sodium and chloride concentrations are lowest in the interior, the external potassium concentration is most easily altered. The physiological concentration of potassium $(4 \mathrm{mM})$ is low as compared to sodium $(18 \mathrm{mM})$ and chloride $(6 \mathrm{mM})$, making it more susceptible to large percent changes in concentration and therefore large changes in the its reversal potential.

\subsubsection{Excitability and refractory periods}

Neuronal excitability is a function of both the resting membrane potential as well as the threshold for firing. This threshold is determined by the voltage gated response of sodium channels as well as the electrochemical gradient for sodium. If sodium gradients are diminished they will cause the threshold to rise to higher voltages making the cell less excitable, providing some amount of negative feedback on continual firing. This mechanism is widely accepted as the means by which some bursting neurons intermittently cease their activity (Catterall, 2000; Hodgkin \& Huxley, 1952).

After spike initiation, sodium influx, and depolarization, voltage gaited potassium channels open and release potassium from the cell, thus repolarizing the membrane. The rate of this repolarization is determined by the electrochemical potassium gradients and potassium conductance. If either of these mechanisms are impaired the duration of the depolarization is lengthened, resulting in increasing transmitter release. The magnitude of the hyperpolarization can also become diminished and cause a reduced or eliminated relative 
refractory period. On the other hand, in neurons with inactivating sodium channels, the loss of hyperpolarzation may lead to a prolonged absolute refractory period and therefore reduced potential for fast action potential generation (Catterall, 2000).

\subsubsection{Synaptic release and response}

Synaptic release is initiated when an action potential enters a buton and depolarizes its membrane. This depolarization activates voltage-gated calcium channels and allows calcium to enter the buton, producing a fusion of vesicles with the plasma membrane and the release of transmitter into the synaptic cleft. The amount of calcium entering the buton depends on the duration of depolarization by the action potential as well as the electrochemical drive forcing calcium into the cell. Therefore low extracellular levels of calcium work to inhibit synaptic release.

As stated in the previous section the reversal potentials for all mixed ion channels are approximated by the weighted average of the ions they conduct. This treatment can be applied to any ion channel. Here we will discuss the effects on the AMPA and GABAa receptors. The AMPA channel is in general excitatory, and it conducts calcium, potassium, and sodium. Decreased potassium gradients will make these channels more excitatory while decreased calcium and sodium gradients will lead to decreased excitation (Kager et al., 2000).

The GABAa receptor is permeable to both chloride and bicarbonate. The reversal is generally very low due to the chloride reversal potential. Maintenance of the chloride gradient is essential to produce strong inhibitory responses. If the breakdown in this gradient is significant, chloride can flow out of the cell upon receptor activation leading to excitation. As mentioned above the chloride gradient is intimately linked to the potassium gradients. Therefore, losses in potassium gradients are once more implicated in making neural networks more excitable.

\subsubsection{Edema}

Ionic imbalances can lead to transient osmotic gradients between neurons and glia and the extracellular space. These gradients are diminished by the flow of water towards regions of high concentration. When concentrations are higher in cells, as is the case during metabolic malfunction, or when glia absorb extracellular potassium, water movement into the tissue results in edema. This swelling can have detrimental effects on further potassium clearance through diffusion (Somjen 2004; Ragaišis, 2002; Park et al., 2008).

\subsubsection{Acidosis and alkalosis}

Acidosis (a lower than normal $\mathrm{pH}$ ) and alkalosis (a greater than normal $\mathrm{pH}$ ) can affect neuronal excitability. In general, acidosis lowers excitability, and alkalosis increases excitability. This is because $\mathrm{H}^{+}$ions directly modulate ion channels, both voltage gated and ligand gated.

$\mathrm{H}^{+}$ions inhibit all voltage gated ion channels, but not equally. If all channels were equally inhibited, there would be no impact on excitability, because inward currents would still balance outward currents. However, calcium channels (the most inhibited) and sodium channels are inhibited much more strongly than potassium channels. In fact, in $\mathrm{pH}$ ranges that are consistent with life, potassium is relatively unaffected by $\mathrm{H}^{+}$ions. This means that 
an increased concentration of $\mathrm{H}^{+}$ions (acidosis) inhibits inward currents (such as $\mathrm{Na}^{+}$and $\mathrm{Ca}^{2+}$ ) while leaving outward currents unaffected. The overall net result is more outward current relative to inward current, and a hyperpolarized neuron. This is consistent with lower excitability. On the other hand, an absence of $\mathrm{H}^{+}$ions (alkalosis) would boost inward currents, and somewhat depolarize the cell, leading to excitability. In addition, at the synapse, an increased flow of inward $\mathrm{Ca}^{2+}$ results in an increased release of neurotransmitters, which also helps explain why alkalosis causes excitability(Somjen, 2004). $\mathrm{H}^{+}$ions also impact ligand-gated ion channels. Glutamate modulated ion channels are depressed by $\mathrm{H}^{+}$, resulting in inhibition. In contrast, GABA modulated ion channels are enhanced by $\mathrm{H}^{+}$, also resulting in inhibition (Somjen, 2004).

\section{Epilepsies}

\subsection{Idiopathic}

Out of the vast array of proteins expressed in the brain, only a small number are responsible for epilepsies. The complexity of the brain and its behavior may lead one to expect a greater variety of disorders. However, insensitivity to most parameters, redundancy, and the fact that phenotypes can only survive as long as their seizures remain transient, all limit the range of genetic variation that can lead to seizure disorders.

Familial hemiplegic migraines are associated with mutations in the genes encoding sodium/potassium ATPase and in rare cases patients exhibit epileptic seizures (Ashmore et al., 2009; Gallanti et al, 2008). With decreased pumping, normal neuronal activity results in the general loss of all ionic gradients. Complete loss of gradients will however more likely lead to spreading depression and perhaps migraine rather than seizures. In order to produce sustained seizure activity the pumps must remain at least partially functional.

In a similar fashion, without glucose, oxygen, and an intact metabolic system to produce ATP, sodium/potassium pumps are incapable of maintaining the resting potential and cells quickly depolarize once they drift above threshold and begin firing action potentials. If ATP is not produced in a relatively short time, loss of mitochondrial gradients as well as elevated calcium concentrations will initiate apoptotic pathways. There is then the potential for the creation of lesions whose spatial extent depends on the scale, duration, and severity of the metabolic deprivation.

Even though there are a large number of ion channels, only a small number have been found to be directly responsible for epileptic syndromes. Generalized epilepsy with febrile seizures plus (GEFS+) encompasses a number of disorders including severe myoclonic epilepsy of infancy (SMEI), border-line SMEI (SMEB), and intractable epilepsy of childhood (IEC) (Kapur 2002; Lossin et al. 2002). In total, there are four types of GEFS+, each defined by its causative mutation (Lossin et al., 2002). Three types of GEFS+ include mutations to either the alpha or beta subunit of the NAv1.1 or Nav 2.1 sodium channels (FujiwaraTsukamoto et al., 2004; Rosenberg et al., 1997). There are a number of different direct effects on sodium channel conductance, including changes in inactivation and frequencydependent rundown. The loss of inactivation removes a strong governing effect on neuronal firing, increasing afferent signaling as well as ionic accumulation around the effective cells. The former obviously leads to greater excitation, but would also lead to greater inhibitory output. Ionic accumulation not only increases due to the increased activity, but also because the loss of inactivation allows higher sodium currents to keep the cell from repolarizing. This windowing effect will be discussed in greater detail below. 
Epilepsies can arise from disfunction to the inhibitory mechanisms. Mutations to $\mathrm{GABA}_{\mathrm{A}}$ receptors are implicated in GFES+ type 3. In neuronal networks with these mutations, inhibitory currents are one-tenth of their normal strength. The direct loss of inhibition should be weakly compensated for by the reduction of potassium flowing out of the $\mathrm{KCl}$ cotransporters, as decreased potassium flow should reduce the potassium accumulation outlined above. This effect is most likely insignificant in comparison to the loss of inhibition and potential for uncontrolled excitatory discharge.

As previously mentioned, cotransporters are important for establishing chloride gradients. Unlike sodium, calcium, and potassium, chloride has no other means to produce a gradient than by utilizing cotransporters. There is evidence that reexpression of juvenile sodiumpotassium-chloride cotransporters occurs after trauma (Shimizu-Okabe et al., 2007; Payne, 1997), and upregulation of the gene for the sodium-potassium-chloride cotransporter has been demonstrated in epileptic tissue (Shimizu-Okabe et al., 2007). In the adult form these cotransporters moves both potassium and chloride out of the cell functionally increasing the strength of post synaptic inhibitory currents. The juvenile form moves potassium sodium, and chloride into the cell. This juvenile form diminishes the strength of GABA and its reversal potential can even lie above the resting potential resulting in GABAergic excitation. On the other hand decreasing the potassium and sodium reversal potentials causes a relative inhibitory effect on the neurons.

It is interesting to note that there is no significant evidence that mutations to potassium channels leads to seizures disorders. It is easy to imagine that any source of significant change in potassium conductances would have a severe effect on both the resting potential, excitability, and synaptic strength. Perhaps it is because of this sensitivity that phenotypes with potassium channel dysfunction are not viable.

\subsection{Symptomatic}

Symptomatic epilepsies are caused by lesions, malformations, and tumors. Although these seizures are due to identifiable structures, the specific cellular alterations and therefore etiologies are quite different. These alterations can include structural and functional changes due to direct mechanical insult or genetic regulation due to more complex processes such as immunological responses.

Brain lesions can either be highly localized as in the case of traumatic injury or ischemia, spread across elements of extensive neuronal circuits like the limbic system, or present diffusely as in a systemic metabolic crisis. The tissue within and surrounding a lesion is different from normal brain. In certain severe lesions complete neuronal death occurs, leaving microglia and astrocytes to occupy the interior of the lesion. In other cases a reduced number of neurons remain within the lesion. In these cases, the behavior and morphology of these neurons can change. In the next section we will discuss experimental models aimed at investigate these post trauma alterations. They suggest that seizures may more easily arise and persist due to reduced potassium buffering by glial cells affected by the trauma, perforation of the BBB, and subsequent immune response.

Tumors, malignant and benign, can induce epileptic seizures. In many cases, these seizures appear to originate from the borders of the tumor (de Groot et al., 2010) and may not even present until the tumor is removed. In other cases the cells within the tumor are functionally active and may play a role in seizure generation themselves (de Groot et al., 2010). There are numerous types of tumors that can result in epileptogenesis. Some gliomas expand into the 
surrounding parenchyma by releasing large quantities of glutamate. This glutamate excites local neurons, depolarizes them, inducing high levels of activity that eventually leads to neuronal death. As the neurons die off the cancer can expand into the vacated space. Prior to cell death the excessive excitation, in tandem with other modifications to the tissue can lead to seizure generation.

Seizures caused by malformations, such as cortical dysplasia, are associated with the presence of abnormal cells in the region of the malformation. The effects of these morphological and structural abnormalities on seizure generation is not well understood. However as has been discussed above in the context of edema, relatively large cellular volume can lead to reduced potassium buffering by the glial network. Similarly, structural changes associated with other diseases such as Sturge Weber syndrome can cause seizures. In this case vasculature malformations exerts stresses on the local neuronal and glial tissue. As is often the case, it is straightforward to identify qualitative mechanisms whose actions could lead to epileptic behavior. However we are often presented with opposing mechanisms whose combined effects cannot be easily ascertained. As these systems are complex it is often impossible to analytically determine which effects will dominate and further investigation via computational modeling or experiments are necessary.

\section{Models}

As already mentioned, the IRS and neuronal dynamics in general can produce complicated dynamics. Therefore altered function of a specific mechanism cannot always be extrapolated to overall behavior. To this end it is often necessary to perform experiments or computational simulations.

\subsection{Experimental models}

\subsubsection{In vivo}

Most experiments aimed at understanding the electrophysiology of epilepsy are performed in rodent models. Most in vivo models involve producing damaged, or lesioned, neuronal circuits. Whether it be through electrical, chemical, or mechanical insult the resulting brain will show significant and stereotypical damage to susceptible regions and cell types. As in human epilepsy, the development of chronic seizures in these animal models is not immediate. Epileptogensis is due to changes to the neuronal circuits that involve apoptotic and necrotic cell death, astrocyte and microglia migrations, and complex alterations to neuronal and astrocytic function. A complicated set of observable changes is well documented in the literature and we will only discuss a small number of them here.

It has recently been proposed that epileptogenesis occurs at least in part due to a breakdown in the BBB trauma, infection, or ischemia (Friedman et al., 2009). Animal models demonstrate that neuronal tissue undergoes a set of stereotyped alterations in response to albumin entering the extracellular space through the breeched BBB. First, there is a reduction in the expression of Kir4.1 channels in glial endfeet. These channels as discussed above are one of the essential features of glia that allow them to buffer potassium from the extracellular space. Secondly, there is a reduction in the expression of gap junctions in glial cells. These gap junctions link together glial cells forming a syncytium that facilitates potassium buffering as discussed previously. As with a reduction in Kir channels the impaired syncytium limits the ability of the glia to remove potassium from the extracellular 
space. Experiments on genetically modified animals confirm that these effects facilitate the generation of seizures (Binder \& Steinhäuser, 2006; Djukic et al., 2007; Wallraff et al., 2006). Dysfunctional glutamate metabolism in glial cells is also implicated in epileptogenesis following trauma. As discussed above one of the primary functions of glial cells is to recycle glutamate. In addition glial cells can also release glutamate on their own and may play a more central role in seizure generation than previously thought (Tian et al., 2005). Of course tonic, or large releases of glutamate will lead to increased activity and therefore strain the IRS. Finally, transformed astrocytes release more inflammatory mediators than normal glia. The release of inflammatory mediators has been implicated in epiletogenesis (Hinterkeuser et al., 2000).

All in all these modifications to the glial tissue result in a compromised potassium buffering system, with the potential for greater excitation through poorly regulated glutamate trafficking and increased inflammatory interference.

\subsubsection{In vitro}

There is a large array of experimental models for seizures, that are in large part based on what is observed in epileptic seizures. Most experiments are carried out in brain slice preparations, usually dissected from rats or mice. Experimental models are designed to subject the neuronal tissue to conditions with combinations of ionic imbalance, excess excitation, reduced inhibition, or hyperactivity. For brevity, we will only discuss a few of these models and the role ionic imbalance plays in the behaviors seen.

Several seizure models are based on asserting ionic imbalances. Increasing extracellular potassium leads to the effects outlined above and is in almost all ways excitatory (Jensen \& Yaari, 1997). Substantial increases in potassium can directly lead to seizure-like activity, and slightly elevated potassium is often used to promote activity in other models. Seizures can also be induced by reducing the extracellular calcium concentrations(Bikson et al., 2003). Both of these models are expected to exhibit reduced synaptic transmission, suggesting that these seizures are largely mediated by concentration dynamics.

Seizures are generally thought to be at least partially due to an imbalance between excitatory mechanisms and inhibitory mechanisms. A number of models attempt to produce these conditions by pharmacologically reducing inhibition with such agents as bicuculine or picrotoxin, or increasing excitatory mechanisms with agents such as carbachol. Not surprisingly these models produce uncontrolled excitatory output and seizure like events. Though the primary reason for these uncontrolled events is a tilting towards excitation, the dynamics of the seizures are undoubtedly influenced by the ionic imbalances produced by the hyperactivity.

There is some debate as to what degree inhibitory mechanisms are compromised in epileptic tissue. Some experimental models, such as 4-aminopyridine, are based on the assumption that it is not inherent loss of inhibition that leads to seizures but temporary loss due to the interactions between the networks of cells (Ziburkus et al., 2006). There it was demonstrated that prolonged seizures-like events can occur with intact inhibition. Excitatory output did not peak due to lack of activity in the interneuron network, but rather was accompanied by the interneurons being forced into a depolarized state through excess activity. This state, known as depolarization block, sets a threshold on cellular, especially inhibitory, output. Depolarization block, as will be discussed in the next section is influenced by, or even caused by, ionic imbalance. In addition to influencing the membrane potential, ionic imbalance will also shift the reversal potentials for synaptic transmission. Understanding 
these effects may greatly enhance our understanding of the balance between excitation and inhibition during pathological states.

\subsection{Mathematical models}

Computational models are an efficient and relatively transparent method to investigate neuronal dynamics. For the sake of simplicity most computational models of neurons do not incorporate changes to ionic concentrations, even though one could argue that even small changes in the ionic distributions can influence network activity, and may in fact be used by the healthy brain to prime or suppress the responsiveness of the network (Frohlich et al., 2010). Either way there is no question that one must include concentration dynamics in order to understand the dynamics of seizures. This has been done on several different levels of complexity, from modeling individual neurons to networks of neurons (Somjen, 2004; Bazhenov et al., 2004; Cressman, 2009; Barreto \& Cressman, 2011). Models are always approximations to physical systems, therefore results and predictions must be interpreted within the narrow scope of the models focus.

Although it is often possible to ascertain the general mechanisms involved in an epileptic disorder, the non-linearity of these mechanisms as well as coupling between different mechanisms often rules out a simple linear analysis. In these cases it is most often necessary to mathematically formulate these mechanisms and computationally evolve their dynamics in order to determine their effects.

\subsubsection{Single cell models}

Single cell models can either be optimized for the most transparent analysis, or be extremely complex in order to understand the detailed interactions of pumps, channels and transporters. Both of these approaches give their own insights into seizure dynamics.

The most detailed neuronal models include multiple compartments for the soma, dendrite, extracellular space, and glia (Kager et al., 2007). These models include numerous ionic channels, pumps, and cotransporters for potassium, sodium, chloride, and calcium, inserted at different densities for different compartments. This sophisticated model allows a detailed inspection of the mechanisms responsible for sustained after discharges.

One of the main results of (Kager et al., 2007) relates to the inactivation of sodium channels. They showed a strong relationship between seizure susceptibility and the permeability of the persistent sodium current. In their model, prolonged self sustained after discharges are maintained by a nearly balanced outward flow of potassium and inward flow of sodium. If sodium channels inactivate, this balance cannot be maintained and potassium flow will outstrip the sodium, and repolarize the cell. However with persistent sodium currents or incomplete inactivation of transient sodium this near balance can sustain long-term depolarized states. This incomplete inactivation is called windowing, due to the overlap between activation and inactivation curves for the transient sodium channels. Seizures finally subside as pump rates increase and currents decrease as ions approach equilibrium. They also show that the ionic imbalance produced leads to sustained depolarization and if severe enough, depolarization block.

On the other hand extremely simple models of single neurons have been employed to investigate the slow modulating effects of ionic changes with less attention paid to the individual currents responsible for the membrane potential and concentrations (Cressman et 
al., 2009). In this study the authors investigated how seizures can arise from alterations to the IRS. The model was designed to replicate high potassium in vitro experiments described above. As the bath concentration in the model is increased to values used to produce seizures in experiments this model supports stable oscillatory behavior that could be the basis for seizures seen in experimental models. This model has also been utilized to investigate the role of depolarization block in seizure dynamics (Barreto \& Cressman, 2011). Here the model was used to determine the parameters for glial function and potassium diffusion that produces the different patterns of neuronal firing and depolarization block seen in experimental models.

\subsubsection{Network models}

Of course seizures are the manifestation of activity that emerges from networks of interacting cells. There are several mechanisms that couple the activity of cells, namely synaptic transmission and extrasyaptic neurotransmitter release, gap junctions, and ionic flow through diffusion in the extracellular space as well as through the glia syncytium. A number of models incorporate these mechanisms (Frohlich et al., 2010; Park et al., 2008; Ullah et al., 2009).

In all of these models extracellular potassium concentrations play a role in coupling the activity of neighboring cells. This effect is investigated to varying degrees in a number of computational models(Park et al., 2008, Frohlich et al., 2010; Ullah et al., 2009). Durand's work focuses on the effect of extracellular coupling to synchronize bursting activity in networks of synaptically uncoupled neurons. Their main results suggest that larger networks require greater extracellular coupling to synchronize. Their work suggests that small networks with limited synaptic connectivity are more susceptible to synchronization.

The works of Frohlich et al. and Ullah et al. focuses on the effect of potassium on the excitability and stability of activity within neuronal networks. Both of these models investigate one of the most difficult aspects to understanding seizures. Namely, how do networks only occasionally support seizure like states? In Ullah et al., 2009 the authors demonstrate that finite sustained activity, such as that implicated in short term memory (Gutkin et al., 2001), can be stabilized or destabilized by changes in glial function.

Frohlich et al. present a different form of multistability in networks of neurons with ionic and synaptic coupling. They demonstrate that networks can be forced into one of two dynamic states by varying the degree of perturbation. The two stable dynamic states in their model represent normal and pathological function. They demonstrate that the transition between these two states can be tuned through modifications to their ionic regulatory system. Both of these models implicate glial function and ionic balance in general as powerful modulators of network function as well as possible culprits in establishing pathological conditions.

\section{Anti epileptic drugs}

There are over 50 million diagnosed cases of epilepsy throughout the world, and many people are able to live without seizures thanks to the availability of a wide array of antiepileptic drugs. The first rudimentary antiepileptic drug was introduced in the mid1800s, and numerous more have been developed since, each acting via a slightly different 
set of mechanisms that lend them their anti-convulsant capacity. However, despite the advancement in antiepileptic drugs, as many as 30\% of patients suffer from refractory epilepsy, meaning that no combination of drugs render them completely seizure free. This fact, along with an array of serious negative side-effects conferred by some drugs, provides incentive for continued research in AED development.

The basic goal of AEDs has been to dampen excitatory networks (or amplify inhibitory networks) in seizures without impacting day-to-day healthy behaviors. Broadly speaking, there are four major categories of anticonvulsant mechanisms. Most of these mechanisms are firmly rooted in basic ionic processes. Unfortunately for the scientists studying AEDs, in many cases these drugs do not have a single mechanism, but rather rely on multiple mechanisms that could be used to explain the anticonvulsant behavior. The majority of AEDs modulate sodium ion channel activity. Another group of AEDs modulate calcium ion channel activity. Both these mechanisms are effective in part because they limit Sustained High-Frequency Repetitive Firing (SRF). A third group comprises those AEDs that modulate the GABAergic system, particularly through the GABAr receptor. Finally, the fourth category of AEDs limit the effectiveness of the excitatory neurotransmitter glutamate. There are a variety of additional minor mechanisms of AEDs, including modulation of potassium channels, inhibition of carbonic anhydrase, modulation of hyperpolarization-activated cyclic nucleotide-gated $(\mathrm{HCN})$ channels, and even modulation of $\mathrm{Na}+/ \mathrm{K}+$ ATPase pumps.

\subsection{Inward cations}

\subsubsection{Voltage-gated $\mathrm{Na}+$ channels}

Many AEDs selectively decrease current through voltage-gated sodium channels. These AEDs include carbamazepine, lamotrigine, oxcarbazepine, phenytoin, topiramate, valproic acid, and zonisamide, and possibly a new drug, Carisbamate (Liu, 2008). Evidence strongly supports the idea that these AEDs bind to voltage-gated sodium channels when they are in an inactivated state brought on by an action potential (AP). Because these AEDs bind voltage-gated sodium channels only in the inactivated state, they become more bound (and thus more effective) during repetitive APs. This use-dependent effect is critical to these AEDs having increased activity during rapid firing, as in seizure activity, but little or no effect during non-pathologic neuronal firing.

Once bound, these AEDs delay the sodium channel recovery from an inactivated state to a resting state and shift gating to a more negative voltage. For example, a normal voltagegated sodium channel may recover from the inactivated state in an average of $2 \mathrm{~ms}$, but in the presence of phenytoin this recovery make take as long as 90ms (Delorenzo \& Sun, 2002). With the sodium channel in an inactivated state, the neuron becomes refractory to subsequent APs until the AED becomes unbound. In this way, AEDs that modulate sodium channels significantly reduce SRF, which is thought to be a major component of seizure formation and spread. Hyperpolarization of the membrane removes all bound AEDs and allows the sodium channel to fully recover to a resting state. While most drugs that modulate voltage-gated sodium channels utilize this mechanism, there are slight variations from drug to drug. For example, while Topiramate modulates voltage-gated sodium channels in a voltage and use-dependent way, it does not fully block SRF (White, 2002). It is also believed that each drug acts at a different binding site on the sodium channel protein.

These AEDs act in a similar manner as the frequency dependent rundown mechanism compromised in GEFS+ type I. The role of sodium inactivation as a control parameter for 
seizure susceptibility is also highlighted by the models of Somjen et al. There they suggest that the seizure length is very sensitive to the persistent sodium channel conductivity. Their and other models show that it is the persistent or windowed transient sodium channels that enable the self regenerating discharges. Therefore the main action of these drugs is most likely not the direct reduction on excitatory output, rather it is through limiting the self regenerative period for the active neuron itself.

\subsubsection{Ca++ channels}

Many AEDs modulate voltage-gated calcium channels, all having the effect of inhibiting calcium current. However, different AEDs target different calcium channels. For example, lamotrigine inhibits high-voltage gated N/P type calcium channels, which are associated with neurotransmitter release at the synapse. By inhibiting these channels, lamotrigine is probably diminishing neurotransmitter release. Similarly, levitaracetam inhibits N-type $\mathrm{Ca} 2+$ channels. However, by contrast, Succinimides (such as thosuximide), valproic acid, phenytoin, and zonisamide have been shown to inhibit T-type calcium channels, and are widely used for their anti-absence affects. T-type calcium channels are implicated in the generation and spread of bursts, particularly in absence seizures (Kito et al., 1996).

In addition to inhibiting T-type calcium channels, phenytoin is also thought to inhibit calcium uptake into mitochondria, which serve as a buffering system for intracellular free calcium. This inhibition can increase the intrasynaptosomal $\mathrm{Ca}^{2+}$ and cause hyperexcitability through an increase in miniature end-plate potentials. In fact, phenytoin is known to cause hyperexcitability at high concentrations, a reversal from its normally anticonvulsant behavior. This buildup of free calcium in the intrasynaptosomal space may explain this aspect of the drug (Delorenzo \& Sun, 2002).

Studies suggest that topiramate targets L-type high-voltage calcium channels and decreases the peak current through the channel. In addition, it has been suggested that gabapentin inhibits high-voltage calcium channels with a particular specificity to the a2d subunit. This subunit specificity allows gabapentin to modulate some neuronal types more than others (Rogawski, 2002).

\subsection{Synaptic modulation}

\subsubsection{GABAergic inhibition}

As GABA is the primary inhibitory neurotransmitter in the brain, it is a common target for AED drugs. There are two primary ways that AEDs can modulate GABAergic transmission: 1) By modulating either $\mathrm{GABA}_{\mathbf{A}}$ or $\mathrm{GABA}_{\mathbf{B}}$ receptors, or 2) By increasing the amount of GABA in the synapse.

Most drugs that modulate GABA receptors do so via the $\mathrm{GABA}_{\mathrm{A}}$ receptor. Drugs such as benzodiazepines, topiramate, and barbituates such as phenobarbital allosterically enhance the $\mathrm{GABA}_{\mathrm{A}}$ receptor. Since the $\mathrm{GABA}_{\mathrm{A}}$ receptor is linked to chloride ion channels, enhancing these $\mathrm{GABA}_{\mathrm{A}}$ receptors enhances the chloride ion current, hyperpolarizing the neuron. These chloride channels open in bursts that have a short, medium, or long duration (corresponding to $0.5 \mathrm{~ms}, 2.5 \mathrm{~ms}$, and $7.3 \mathrm{~ms}$ ) (MacDonald, 2002). Phenobarbital will increase burst time (favoring longer bursts) without impacting burst frequency (Olsen, 2002). Conversely, benzodiazepines will increase burst frequency without impacting burst time (MacDonald, 2002). Despite subtle differences in mechanism, the end result is the same: an increase in 
chloride ion current. Barbituates are not widely prescribed as anti-convulsants because of sedative side effects. Benzodiazepines are largely free of these side effects, probably because they target a specific sub-type of $\mathrm{GABA}_{\mathbf{A}}$ receptors, limiting their effect throughout the brain. Barbituates are non-selective, and thus widely effective in all areas of the brain. This probably explains the notable sedative side effects compared to benzodiazepines (Olsen, 2002).

Presynaptic $\mathrm{GABA}_{\mathrm{B}}$ receptors inhibit calcium channels (which prevents neurotransmitter release, while postsynaptic $\mathrm{GABA}_{B}$ receptors open potassium channels (which hyperpolarize the neuron). Baclofen is a non-selective $\mathrm{GABA}_{\mathrm{B}}$ agonist, and so increases activity at both presynaptic and postsynaptic receptors. This can result in both anticonvulsant, and pro-convulsant behaviors. The pro-convulsant behaviors are thought to be caused by the presynaptic $\mathrm{GABA}_{\mathrm{B}}$ receptors, which inhibit neurotransmitter release. While baclofen results in a reduction of excitatory neurotransmitter release, it can also reduce GABA release from inhibitory neurons, which play an important role in seizure prevention. There is some evidence that gabapentin modulates postsynaptic $G_{A B A}$ receptors, however this is still debated. By activating only postsynaptic receptors, the pro-convulsant effects of baclofen are eliminated (Meldrum \& Rogawski, 2007).

In addition to modulating GABA receptors, some AEDs also modulate the amount of GABA in the synapse. Tiagabine blocks the uptake of GABA back into the neuron by blocking the GABA transporter, GAT-1 (Giardina, 2002). This has the effect of leaving GABA in the synapse longer, prolonging inhibitory postsynaptic potentials. Valproic acid increases GAD activity, an important synthesizing enzyme for GABA. Increased GABA synthesis results in increased GABA in the brain (Löscher, 2002). Finally, vigabatrin is an irreversible GABA-T inhibitor. GABA-T catalyzes the conversion of GABA to succinic semialdehyde, and so reduces GABA levels in the brain. By inhibiting GABA-T, GABA is increased in the brain (Ben-Menachem, 2002).

\subsubsection{Glutamatergic transmission}

Glutamate is the most abundant excitatory neurotransmitter, and acts by binding to NMDA, AMPA, and kainate receptors at the synapse. Some AEDs target these receptors as a means to limit excitatory transmission. For example, felbamate and valproic acid act as antagonists of the NMDA receptor, thus inhibiting glutamatergic transmission. This is indirectly an ionic effect, because an activated NMDA receptor opens a nonspecific cation pore, which allows $\mathrm{K}^{+}$to flow out of the cell along its gradient, and $\mathrm{Na}^{+}$and $\mathrm{Ca}^{2+}$ to flow in. This has the effect of depolarizing the cell, contributing to excitability. Many traditional AEDs that inhibit glutamatergic transmission have intolerable side effects, and so are not used. However, felbamate is a drug that is well tolerated, despite acting on NMDA receptors. It has been shown that felbamate is a much more successful antagonist of NMDA receptors containing NR2B subunits, as opposed to NR2A. NR2B subunits are mainly found in forebrain areas, while NR2A subunits are found throughout the brain. Felbamate's tolerability may be owed to it is focused antagonism of NMDA in the forebrain areas of the brain, which play an important role in seizure generation, while other areas are left relatively unaffected (Rogawski, 2002). Kainate and AMPA receptors are also targets for AEDs. For example, topiramate is thought to inhibit Kainate receptors.

\section{$5.3 \mathrm{~K}+$ channels}

While voltage-gated sodium and calcium ion channels have long been targets of AEDs, targeting voltage-gated potassium channels is relatively new. A new AED, Ritagabine, has 
been shown to modulate voltage-gated potassium channels. Research also indicates that lamotrigine gets some anti-convulsant properties by modulating potassium channels (Czuczwar et al, 2010).

Ritagabine increases current through the Kv7.2-7.3 subunits of the voltage-gated M-type K+ channel. This is done by lowering the activating potential required to open these ion channels down to levels close to the resting membrane voltage. This is either done by stabilizing the M-type channels in their open state, or destabilizing them in their closed state. Evidence suggests that the binding site is exposed when the channel is in the open state, and thus the channels are stabilized in their open state (Czuczwar et al, 2010; Schener et al., 2005). Binding of Ritagabine probably interferes with the closing of the channel until lower voltages.

Opening these potassium channels allows potassium to flow out of the cell due to its gradient. This has the effect of lowering the membrane potential back towards resting or even hyperpolarizing levels, thus reducing excitability. Ritagabine has been shown to hyperpolarize neurons, which supports this explanation for some of its anti-convulsant properties.

\subsection{Carbonic anhydrase and acidosis}

Research suggests that some AEDs, such as topiramate and zonisamide derive their anticonvulsant properties in part from their ability to modulate the $\mathrm{pH}$ in the brain. This is done by inhibiting Carbonic Anhydrase (CA), the enzyme responsible for interconverting $\mathrm{CO}_{2}$ and $\mathrm{H}_{2} \mathrm{O}$ with protons and bicarbonate. The inhibition of CA results in the buildup of $\mathrm{H}^{+}$ ions from the metabolic process, and mild acidosis results. This is significant because the presence of an excess of $\mathrm{H}^{+}$ions modulates ion channel activity, and causes inhibition, as explained previously (Mizra et al., 2009).

\subsection{HCN channels}

There is recent evidence that gabapentin and lamotrigine increase the inward $\mathrm{I}_{\mathrm{H}}$ current in some neurons. The $\mathrm{I}_{\mathrm{H}}$ current is an important part of the ability of cardiac cells and neurons to control rhythmicity. Hyperpolarization-Activated, Cyclic Nucleotide-Gated $\mathrm{K}^{+}(\mathrm{HCN})$ ion channels are responsible for the $\mathrm{I}_{\mathrm{H}}$ current. As the name would suggest, these ion channels are hyperpolarization activated, and allow $\mathrm{Na}^{+}$and $\mathrm{K}^{+}$to flow into and out of the cell respectively $\left(\mathrm{K}^{+}\right.$is four times more permeant than $\left.\mathrm{Na}^{+}\right)$(Clapham, 1998). While the role of $\mathrm{HCN}$ channels in cardiac tissue is more clearly understood, the distribution and effect on neurons in the brain is still a matter of research and debate. However, it is clear that $\mathrm{I}_{\mathrm{H}}$ plays a role in both pyramidal cells and inhibitory interneurons in the hippocampus, and may help to control the synchronized firing that is important for information processing. Studies show that HCN channels are active at the resting membrane potential for many hippocampal neurons. This net inward flow has the effect of depolarizing the neuron slightly, thus increasing excitability. At first glance, it would seem that $\mathrm{I}_{\mathrm{H}}$-induced excitability of inhibitory interneurons would cause overall inhibition, while $\mathrm{I}_{\mathrm{H}}$-induced excitability in pyramidal cells would cause excitation. However, research shows that the overall effect of increased $\mathrm{I}_{\mathrm{H}}$ currents is inhibition.

The reason for this is more obvious in interneurons. An increase in $\mathrm{I}_{\mathrm{H}}$ currents in inhibitory interneurons has a slight depolarizing effect by about $5-10 \mathrm{mV}$ (George et al., 2009), making it easier for these cells to fire. In fact, research indicates that this is one way gabapentin and 
lamotrigine get their anti-convulsant properties (Peng et al., 2011). Despite having a similar effect, lamotrigine and gabapentin modulate $\mathrm{I}_{\mathrm{H}}$ currents differently. Lamotrigine changes the activation potential of HCN channels (Poolos et al., 2002), while gabapentin increases the conductance while leaving the voltage gating unchanged (Surges et al., 2003).

The impact on pyramidal CA1 cells is perhaps less obvious. Interestingly, increasing $\mathrm{I}_{\mathrm{H}}$ current in CA1 pyramidal cells has a nonlinear effect. Increasing $\mathrm{I}_{\mathrm{H}}$ currents in conjunction with weak, subthreshold Excitatory Post-Synaptic Potentials (EPSPs) results in an overall excitatory effect. The peak voltage achieved by the EPSP is increased by the $\mathrm{I}_{\mathrm{H}}$ currents, though still not enough to cause an action potential. However, strong subthreshold EPSPs have an overall inhibitory effect. The peak voltage achieved by these stronger EPSPs is actually lessened by $\mathrm{I}_{\mathrm{H}}$ currents. If increased $\mathrm{I}_{\mathrm{H}}$ currents have a depolarizing effect on neurons, how does this lead to inhibition? The answer rests with M-type voltage-gated K+ channels. These channels are activated by modest depolarizations of the neuron, and allow $\mathrm{K}+$ to flow out the neuron along its concentration gradient. M-type $\mathrm{K}+$ channels thus have a hyperpolarizing (inhibitory) effect on neurons. Weak EPSPs are not enough to fully activate M-type $\mathrm{K}+$ channels, and excitation results. However, as the EPSPs get stronger, $\mathrm{K}+$ channels are activated, and inhibition results. Thus, the overall impact of gabapentin and lamotrigine is to make it more difficult for EPSPs to reach the action potential threshold, and thus is inhibitory (George et al., 2009).

\section{$5.6 \mathrm{Na}+\mathrm{K}+$ ATPase pumps}

Some research indicates that AEDs, such as phenytoin, can increase the activity of the sodium potassium pump. Increasing the sodium potassium pump draws more potassium into the cell, and forces more sodium out, hyperpolarizing the cell. (Guillaume et al., 1986) There is also some evidence that this effect is dependent on the $\mathrm{K}+/ \mathrm{Na}+$ ratio in the extracellular space. When there is a higher ratio of $\mathrm{K}+/ \mathrm{Na}+$, phenytoin is more effective at increasing $\mathrm{Na}+/ \mathrm{K}+$-ATPase activity. Because extracellular $\mathrm{K}+$ levels can often rise during seizures, this provides a mechanism where phenytoin can modulate seizure behavior without influencing healthy behaviors (Delorenzo \& Sun, 2002). This is one of the few targets which is aimed at interacting with the IRS directly.

\section{Summary}

Although the importance of ion concentrations in seizure dynamics has long been established (Green \& Petsche, 1961), the effects of ionic imbalances are still not well understood. Mounting experimental and computational results are beginning to untangle the role of the IRS in seizure dynamics. These findings suggest that seizure initiation, evolution, and termination are all highly influenced by changes in the ionic balance. The knowledge gleaned from this body of work should be used to better understand current pharmacological agents as well as used to inform new pharmacological targets, and other forms of treatment such as electrical or magnetic stimulation.

We have presented the basic mechanisms responsible for ionic regulation as well as their role in maintaining normal ion concentrations. We also described a number of common interactions between neuronal activity and ionic concentrations that directly influence neuron behavior and signaling. By influencing a wide array of neuronal properties, including, membrane excitability and synaptic potency, ionic imbalance can play a large role in establishing the behavior of networks of neurons. 
In order to further establish the relevance of ionic imbalance in epilepsy we looked at the role of these mechanism in known etiologies. For known idiopathic epilepsies the function of the implicated proteins will often suggest a mechanism for seizure susceptibility. For instance, the loss of sodium channel inactivation may lead to prolonged depolarization and a greater increase in ionic imbalance. Even in diseases where the obvious culprit is inhibitory malfunction the effects of ionic imbalance are still highly relevant to the dynamics of these seizures.

In symptomatic seizures the alterations to neuronal and glial cells in and around lesions lead to a variety of abnormal behavior. Glial cells are highly implicated in many symptomatic seizures. Seizure susceptibility in some of these cases could be due to the diminished ability of glial cells to maintain ionic balance.

We concluded by looking at the major classes of antiepileptic drugs and their effects on the IRS. Though all of these pharmacological agents effect, to some degree, ionic balance, only a few directly act on it.

Experimental and mathematical models strongly suggest that glial malfunction is implicated in seizure dynamics. To this point, there are no drugs targeted at glial function. This could be a promising avenue for new antieleptic agents. Since the IRS composes a coupled system it important to realize that no target is entirely specific. Therefore one cannot expect to solely interact with any one ionic gradient. It is therefore essential to make use of experimental and theoretical models to evaluate the effectiveness of pharmacological manipulations.

\section{Conclusions}

It was our goal to inform the reader of the importance of ionic imbalance in seizures, both to better understand the nature of seizures and their manifestations, as well as to think of better ways to directly interact with the Ionic Regulatory System to maintain normal neuronal states.

\section{References}

Ashmore, L.J., Hrizo, S. L., Paul, S. M., Van Voorhies, W. A., Beitel, G. J., \& Palladino, M. J. (2009). Novel mutations aVecting the $\mathrm{Na}, \mathrm{K}$ ATPase alpha model complex neurological diseases and implicate the sodium pump in increased longevity. Hum Genet 126:431-447. doi: 10.1007/s00439-009-0673-2

Attwell, D., \& Laughlin, S.B. (2001). An energy budget for signaling in the grey matter of the brain. J. Cereb. Blood Flow Metab. 21, 1133-1145.

Barreto, E. and Cressman, J.R.(2011). Ion concentration dynamics as a mechanism for neuronal bursting. Journal of Biological Physics, 37, (3), 361-373, DOI: 10.1007/s10867-010-9212-6

Bazhenov, M., Timofeev, I., Steriade, M., \& Sejnowski, T. J. (2004). Potassium model for slow (2-3 Hz) in vivo neocortical paroxysmal oscillations. Journal of Neurophysiology, 92, 1116-1132. doi:10.1152/jn.00529.2003.

Ben-Menachem, E. (2002). Vigabatrin, In: Antiepileptic Drugs, Levy, R., Mattson, R., Meldrum, B., Perucca, E., p 855. Lippincott Williams \& Wilkins, ISBN 0-7817-23213, Baltimore 
Binder, D.K., \& Steinhäuser, C. (2006). Functional changes in astroglial cells in epilepsy. Glia 54:358 -368.

Butt, A. M., Kalsi, A. (2006). Inwardly rectifying potassium channels (Kir) in central nervous system glia: a special role for Kir4.1 in glial functions. J. Cell. Mol. Med. 10: (1), 3344

Catterall, W.A. (2000). From ionic currents to molecular mechanisms: the structure and function of voltage-gated sodium channels. Neuron, 26:13-25. doi: 10.1016/S08966273(00)81133-2

Clapham, D.E. (1998). Not So Funny Anymore: Pacing Channels Are Cloned. Neuron, 21, 5-7

Cressman, J.R., Ullah, G., Ziburkus, J., Schiff S.J., \& Barreto, E. (2009). The influence of sodium and potassium dynamics on excitability, seizures, and the stability of persistent states: I. Single neuron dynamics. Journal of Computational Neuroscience. doi:10.1007/s10827-008-0132-4.

Czuczwar, P., Wojtak, A., Cioczek-Czuczwar, A., Parada-Turska, J., Maciejewski, R., Czuczwar, S. J. (2010). Retigabine: the newer potential antiepileptic drug. Pharmacological Reports, 62, 211-219. ISSN 1734-1140

de Groot, M., Toering, S.T., Boer, K., Spliet, W.G.M., Heimans, J.J., Aronica, E., \& Reijneveld, J.C. (2010). Expression of synaptic vesicle protein 2A in epilepsy-associated brain tumors and in the peritumoral cortex. Neuro-Oncology 12(3):265-273, 2010. doi:10.1093/neuonc/nop028

Delorenzo, R. \& Sun, D. (2002). Phenytoin and Other Hydratoins; Mechanisms of Action, In: Antiepileptic Drugs, Levy, R., Mattson, R., Meldrum, B., Perucca, E., pp 554-556. Lippincott Williams \& Wilkins, ISBN 0-7817-2321-3, Baltimore

Djukic, B., Casper, K.B., Philpot, B.D., Chin, L.S., \& McCarthy, K.D. (2007). Conditional knock-out of Kir4.1 leads to glial membrane depolarization, inhibition of potassium and glutamate uptake, and enhanced short-term synaptic potentiation. Journal of Neuroscience 27:11354 -11365.

Eccles J.C., Katz B, Kuffler S.W. (1941). Nature of the "endplate potential" in curarized muscle. Journal of Neurophysiology. 4:362-87

Feng, T.P. (1941). The changes in the endplate potential during and after prolonged stimulation. Chinese. Journal of Physiology. 13:79-107

Friedman, A., Kaufer, D., \& Heinemann, U. (2009). Blood-brain barrier breakdown-inducing astrocytic transformation: Novel targets for the prevention of epilepsy. Journal of Epilepsy Research, 85, 2, 142-149, doi: 10.1016/j.eplepsyres.2009.03.005

Fröhlich, F., Sejnowski, T. J., \& Bazhenov, M. (2010). Network bistability mediates spontaneous transitions between normal and pathological brain states. Journal of Neuroscience, 30(32):10734 -10743 doi:10.1523/JNEUROSCI.1239-10.2010

Fujiwara-Tsukamoto, Y., Isomura, Y., Kaneda, K., \& Takada, M. (2004). Synaptic interactions between pyramidal cells and interneuron subtypes during seizure-like activity in the rat hippocampus. Journal of Physiology, 557(3), 961-979.

Gallanti, A., Tonelli, A., Cardin, V., Bussone, G., Bresolin, N., \& Bassi, M. T. (2008). A novel de novo nonsense mutation in ATP1A2 associated with sporadic hemiplegic migraine and epileptic seizures. Journal of Neuroscience 273(1-2):123-6

George, M.S., Abbott, L. F. \& Siegelbaum, S.A. (2009). HCN hyperpolarization-activated cation channels inhibit EPSPs by interactions with M-type K+ channels. Nature Neuroscience 12 (5) 577-584, doi:10.1038/nn.2307 
Giardana, W. (2002). Tigabine; Mechanisms of Action, In: Antiepileptic Drugs, Levy, R., Mattson, R., Meldrum, B., Perucca, E., p 677. Lippincott Williams \& Wilkins, ISBN 0-7817-2321-3, Baltimore

Green, J. D. \& Petsche, H. (1961 a). Hippocampal electrical activity. II. Virtual generators. Electroen. Neurophysiology. 13, 847-853.

Green, J. D. \& Petsche, H. (1961 b). Hippocampal electrical activity. IV. Abnormal electrical activity. Electroen. Neurophysiology. 13, 868-879.

Guillaume, D., Grisar, T., Delgado-Escueta, A.V. (1986) Phenytoin Dephosphorylates the Catalytic Subunit of the $(\mathrm{Na}+, \mathrm{K}+)$-ATPase in $\mathrm{C} 57 / \mathrm{BL}$ Mice. Journal of Neurochemistry, 47, (3), 904-911, doi: 10.1111/j.1471-4159.1986.tb00696.x

Gutkin, B. S., Laing, C. R., Colby, C. L., Chow, C. C., \& Ermentrout, G. B. (2001). Turning on and off with excitation: the role of spiketiming asynchrony and synchrony in sustained neural activity. Journal of Computational Neuroscience, 11(2), 121-134.

Haider, B., Duque, A., Hasenstaub, A.R., \& McCormick, D.A. (2006) Neocortical network activity in vivo is generated through a dynamic balance of excitation and inhibition. The Journal of Neuroscience. 26917):4535-4545.

Hinterkeuser, S., Schröder, W., Hager, G., Seifert, G., Blümcke, I., Elger, C. E., Schramm, J., Steinhäuser, C. (2000). Astrocytes in the hippocampus of patients with temporal lobe epilepsy display changes in potassium conductances. European Journal of Neuroscience 12,(6),2087-2096. doi: 10.1046/j.1460-9568.2000.00104.x

Hodgkin, A.L., Huxley, A.F. (1952). A quantitative description of membrane current and its application to induction and excitation in nerve. Journal of Physiology, 117:500-544.

Hotson, J.R., Sypert, G.W., \& Ward, A.A. (1973). Extracellular Potassium Concentration Changes During Propagated Seizures in Neocortex. Experimental Neurology. 38: 20-26

Jensen, M. S., \& Yaari, Y. (1997). Role of intrinsic burs firing, potassium accumulation, and electrical coupling in the elevated potassium model of hippocampal epilepsy. Journal of Neurophysiology, 77, 1224-1233.

Kager, H., Wadman, J.W., \& Somjen, G.G. (2000). Simulated seizures and spreading depression in a neuron model incorporating interstitial space and ion concentrations. Journal of Neurophysiology,84, 495-512.

Kager, H., Wadman, J.W., \& Somjen, G.G. (2007). Seizure-like afterdischarges simulated in a model neuron. Journal of Computational Neuroscience, 22, 105-128.

Kapur, J. (2002). Sodium channel mutations in GEFS+ produce persistent inward current. Epilepsy Currents, 2 (5), 149-150. doi: 10.1046/j.1535-7597.2002.00055.x

Kito, M., Maeharai, M. \& Watanabe, K. (1996). Mechanisms of T-type calcium channel blockade by zonisamide, Seizure; 5:115-119

Korn, S.J., Giacchino, J.L., Chamberlin, N. L., \& Dingledine, R. (1987). Epileptiform Burst Activity Induced by Potassium in the Hippocampus and its Regulation by GABAMediated Inhibition. Journal of Neurophysiology, 57: 325-340

Lennie, P. (2003). The cost of cortical computation. Current Biology 13, 493-497, doi: 10.1016/S0960-9822(03)00135-0

Liua, Y., Yohrling, G. J., Wang, Y., Hutchinson, T. L., Brenneman, D. E., Flores, C. M., Zhao, B. (2009). Carisbamate, a novel neuromodulator, inhibits voltage-gated sodium channels and action potential firing of rat hippocampal neurons. Epilepsy Research (2009) 83, 66-72. doi:10.1016/j.eplepsyres.2008.09.006 
Lösher, W. (2002). Valporic Acid; Mechanisms of Action, In: Antiepileptic Drugs, Levy, R., Mattson, R., Meldrum, B., Perucca, E., p 772. Lippincott Williams \& Wilkins, ISBN 0-7817-2321-3, Baltimore

Lossin, C., Wang, D.W., Rhodes, T.H., Vanoye,C.G., \& George, A.L. (2002). Molecular basis of an inherited epilepsy. Neuron;34:877-884

Lu, Y., \& Wang, X. (2009). Genes associated with idiopathic epilepsies: a current overview. Neurological Research, 31, 135-143 doi: 10.1179/174313209X393942

Macdonald, R. (2002). Benzodiazepines Mechanisms of Action, In: Antiepileptic Drugs, Levy, R., Mattson, R., Meldrum, B., Perucca, E., p 183. Lippincott Williams \& Wilkins, ISBN 0-7817-2321-3, Baltimore

Meldrum, B. S. \& Rogawski, M.A. (2007). Molecular Targets for Antiepileptic Drug Development. Neurotherapeutics, 4(1): 18-61

Mirza, N., Marson, A.G. \& Pirmohamed, M. (2009). Effect of topiramate on acid-base balance: extent, mechanism and effects. British Journal of Clinical Pharmacology, 68:5, 655-661 doi:10.1111/j.1365-2125.2009.03521.x

Olsen, R. (2002). Phenobarbital and Other Barbituates; Mechanisms of Action, In: Antiepileptic Drugs, Levy, R., Mattson, R., Meldrum, B., Perucca, E., pp 491-492. Lippincott Williams \& Wilkins, ISBN 0-7817-2321-3, Baltimore

Park, E., Feng, Z., \& Durand, D. M., (2008). Diffusive Coupling and Network Periodicity: A Computational Study. Biophysical Journal, 95, 1126-1137. doi: 10.1529/biophysj.108.129239

Payne, J.A. (1997) Functional characterization of the neuronal-specific K1-Cl2 cotransporter: implications for [K1]o regulation. American Journal of Physiology 273:C1516-C1525.

Peng, B., Justice, J.A., Zhang,K., He, X. \& Sanchez, R.M. (2010). Increased Basal Synaptic Inhibition Of Hippocampal Area CA1 Pyramidal Neurons By An Antiepileptic Drug That Enhances IH. Neuropsychopharmacology 35, 464-472, Doi: 0893-133X/10

Poolos, N.P., Migliore, M. \& Johnston, D. (2002). Pharmacological upregulation of hchannels reduces the excitability of pyramidal neuron dendrites, In: Nature Publishing Group [NPG], Available from: http://neurosci nature com . doi: $10.1038 / \mathrm{nn} 891$

Ragaišis V. (2002). Brain contusion: morphology, pathogenesis, and treatment. Medicina 38, (3) http:/ / medicina.kmu.lt 243 /0203/0203-01e.htm., retrieved on 06/05/2011

Ransom, C. B., Ransom, B. R., \& Sotheimer, H. (2000). Activitydependent extracellular K+ accumulation in rat optic nerve: the role of glial and axonal $\mathrm{Na}+$ pumps. The Journal of Physiology, 522, 427-442. doi:10.1111/j.1469-7793.2000.00427.x.

Rogawski, M. (2002). General Principles of Antiepileptic Drug Action, In: Antiepileptic Drugs, Levy, R., Mattson, R., Meldrum, B., Perucca, E., pp 3-18. Lippincott Williams \& Wilkins, ISBN 0-7817-2321-3, Baltimore

Rosenberg, R., Prusiner, S., DiMauro, S., \& Barchi, R. (1997). The Molecular and Genetic Basis of Neurological Disease. City: Butterworth-Heinemann.

Schenzer,A., Friedrich, T., Pusch, M., Saftig, P., Jentsch, T. J., Grötzinger, J. \& Schwake, M. (2005). Molecular Determinants of KCNQ (Kv7) K Channel Sensitivity to the Anticonvulsant Retigabine. The Journal of Neuroscience, 25(20):5051-5060 doi:10.1523/JNEUROSCI.0128-05.2005

Shimizu-Okabe, C., Okabe, A., Kilb, W., Sato, K., Luhmann, \& H.J., Fukuda, A. (2007). Changes in the expression of cation-Cl- cotransporters, NKCC1 and KCC2, during 
cortical malformation induced by neonatal freeze-lesion. Journal of Neuroscience Research 59, 288-295. doi:10.1016/j.neures.2007.07.010

Somjen, G.G. (2004). Ions in the brain: normal function, seizures and stroke. Oxford: Oxford University Press.

Somjen, G.G., Kager, H., \& Wadman, W. J. (2008). Computer simulations of neuron-glia interactions mediated by ion flux. Journal of Computational Neuroscience 25:349-365. doi: 10.1007/s10827-008-0083-9

Somjen, G.G., Kager, H., \& Wadman, W. J. (2009). Calcium sensitive non-selective cation current promotes seizure-like discharges and spreading depression in a model neuron. Journal of Computational Neuroscience 26:139-147. doi: 10.1007/s10827-0080103-9

Stein, V., Hermans-Borgmeyer, I., Jentsch, T. J., Hübner, C. A. (2000). Expression of the $\mathrm{KCl}$ cotransporter KCC2 parallels neuronal maturation and the emergence of low intracellular chloride. Journal of Comparative Neurology, 468,( 1), 57-64. DOI: 10.1002/cne.10983

Surges, R., Frieman, T.M. \& Feuerstein, T.J. (2003). Gabapentin Increases the Hyperpolarization-activated Cation Current $\mathrm{I}_{\mathrm{h}}$ in Rat CA1 Pyramidal Cells. Epilepsia, 44 (2): 150-156

Sykova, E. (2005). Glia and volume transmission during physiological and pathological states. Journal of Neural Transmission 112: 137-147

Tian, G., Azmi, H., Takano, T., Xu, Q., Peng, W., Lin, J., Oberheim, N., Lou, N., Zielke, R., Kang, J., \& Nedergaard, M. (2005). An astrocytic basis of epilepsy. Natural Medicine; 11(9): 973-981. doi: 10.1038/nm1277.

Ullah, G., Cressman, J. R., Barreto, E., \& Schiff, S. J. (2009). The influence of sodium and potassium dynamics on excitability, seizures, and the stability of persistent states: II. Network and glial dynamics. Journal of Computational Neuroscience doi:10.1007/s10827-008-0130-6.

Wallraff, A., Köhling, R., Heinemann, U., Theis, M., Willecke, K., \& Steinhäuser, C. (2006). The impact of astrocytic gap junctional coupling on potassium buffering in the hippocampus. Journal of Neuroscience 26:5438 -5447.

White, H. (2002). Topiramate; Mechanisms of Action, In: Antiepileptic Drugs, Levy, R., Mattson, R., Meldrum, B., Perucca, E., p 721. Lippincott Williams \& Wilkins, ISBN 0-7817-2321-3, Baltimore

Zheng, Wei, Chodobski, \& Adam (2005). The Blood-Cerebrospinal Fluid Barrier. City:CRC Press I Llc.

Ziburkus, J., Cressman, J. R., Barreto, E., \& Schiff, S. J. (2006). Interneuron and pyramidal cell interplay during in vitro seizure-like events. Journal of Neurophysiology, 95, 39483954. 


\section{Part 5}

New Treatments 



\title{
Antiepileptic Medicinal Plants Used in Traditional Medicine to Treat Epilepsy
}

\author{
E. Ngo Bum et al. ${ }^{*}$ \\ ${ }^{1}$ Department of Biological Sciences, Faculty of Science, University of Ngaoundéré, \\ Ngaoundéré, \\ Cameroon
}

\section{Introduction}

Epilepsy is a disease that affects about 40 million people worldwide (Njamshi et al., 2010). In 1968, the prevalence of epilepsy in Africa was about 4.8 to $40 \%$. In 1996, Diop and collaborators reported in Senegal a prevalence of epilepsy of $21 \%$ (Diop et al., 1996). In 2006, Ngoungou and collaborators estimated the prevalence in sub-Saharan Africa to be two or three time highest than the rate in developed world (Ngoungou et al., 2007). In Cameroon, some epidemiological studies on epilepsy have shown that, the prevalence of epilepsy is estimated to vary from 5-136/1000. The highest ones are reported in some villages of the Cameroon Central Province located in the Sanaga and Mbam River Valley (Nchoji Nkwi \& Tioko Ndonko, 1989; Dongmo et al., 2000; Preux et al., 2000; Boussinesq et al., 2002; Kamgno et al., 2003; Dongmo et al., 2004; Prischich et al., 2008). Cameroon is one of the countries most affected by epilepsy in Africa and in the world. Thus, epilepsy is among the major public health problems in Cameroon. In Africa and in Cameroon particularly, phytotherapy in traditional medicine still plays an important role in the management of diseases, mainly among populations with very low income (Geoffrey \& Kirby, 1996). And phytotherapy relies on the use of a wide variety of plant species. Annona muricata Linn (Annonaceae), Annona senegalensis Pers (Annonaceae), Bidens pilosa Linn (Asteraceae), Bryophyllum pinnatum (Lam) Oken (Crassulaceae), Citrus sinenis (Linn) Osbeck (Rutaceae), Clerodendron thomsoniae Balf (Verbenaceae), Daniellia oliveri (Rolfe) Hutch and Dalz (Caesalpiniaceae), Datura stramonium Linn (Solanaceae), Detarium microcarpum Guil et Perr (Caesalpiniaceae), Euphorbia hirta Linn (Euphorbiaceae), Flacourtia indica Willd (Flacourtiaceae), Hymenocardia acida Tul (Hymenocardiaceae), Jatropha gossypiifolia Linn (Euphorbiaceae), Khaya senegalensis A Juss (Desrousseaux) (Meliaceae), Mentha cordifolia Auct (Lamiaceae), Prosopis Africana Guill and Perr (Taub) (Mimosaceae), Ricinus communis Linn (Euphorbiaceae), Securidaca longepedunculata Fres (Polygalaceae), Senna singueana (Delile) Lock 1988 (Caesalpiniaceae), Terminalia glaucescens Planch. ex Benth (Combretaceae), Terminalia mollis Laws (Combretaceae), Tetrapleura tétraptera Taub (Schum Thonn)

\footnotetext{
${ }^{*}$ G.S. Taiwe' ${ }^{2}$, F.C.O. Moto ${ }^{1}$, G.T. Ngoupaye ${ }^{1}$, R.R.N. Vougat ${ }^{1}$, V.D. Sakoue ${ }^{1}$, C. Gwa ${ }^{1}$, E.R. Ayissi², C. Dong'2, A. Rakotonirina ${ }^{2}$ and S.V. Rakotonirina ${ }^{2}$

1 Department of Biological Sciences, Faculty of Science, University of Ngaoundéré, Ngaoundéré, Cameroon

2 Department of Animal Biology and Physiology, Faculty of Sciences, University of Yaoundé 1, Yaoundé,

Cameroon
} 
(Mimosaceae), Trichilia emetica Vahl (Meliaceae) and Vitellaria paradoxa C F Gaertn (Sapotaceae) are plants that are being used empirically in traditional medicine in Cameroon to treat epilepsy and diseases related to the brain like agitations, anxiety, convulsions, dizziness, headaches, insomnia, migraines, pains and schizophrenia according to our traditional Healers and the literature (Abbiw, 1990; Adjanohoun et al., 1984, 1996; Arbonnier, 2000; Berhaut, 1975; Biholong, 1986; Bouquet, 1969; Brenan, 1959; Dalziel, 1937; Hutchinson \& Dalziel, 1958; Iwu, 1993; Joyner, 2004; Malgras, 1992; Mutasa et al., 1990; Nwaiwu \& Akah, 1986; Pousset, 1989; Raponda-Walker \& Silans, 1961; Saulnier, 1998) (Table 1). Though the literature showed a lot of pharmacological studies done

\begin{tabular}{|c|c|c|c|c|c|c|}
\hline $\begin{array}{c}\text { Name of the } \\
\text { plant }\end{array}$ & $\begin{array}{l}\text { Part of the } \\
\text { plant used }\end{array}$ & \begin{tabular}{|c|}
$\begin{array}{c}\text { Form of } \\
\text { the } \\
\text { medicine }\end{array}$ \\
\end{tabular} & Diseases & $\begin{array}{c}\text { Chemical } \\
\text { characterization }\end{array}$ & $\begin{array}{l}\text { Pharmaco- } \\
\text { logical } \\
\text { properties }\end{array}$ & Country \\
\hline \begin{tabular}{|l|} 
Annona \\
muricata
\end{tabular} & Leaves & $\begin{array}{l}\text { Infusion } \\
\text { Decoction }\end{array}$ & $\begin{array}{l}\text { Insomnia, diabetes } \\
\text { Spasms, Fever }\end{array}$ & $\begin{array}{l}\text { Steroid, cardiac } \\
\text { glycosides }\end{array}$ & Antimicrobial & $\begin{array}{l}\text { Cameroon, } \\
\text { Forest areas } \\
\end{array}$ \\
\hline \begin{tabular}{|l|} 
Annona \\
senegalensis
\end{tabular} & $\begin{array}{l}\text { Leaves } \\
\text { Roots }\end{array}$ & Infusion & $\begin{array}{l}\text { Convulsions, Epilepsy } \\
\text { Sterility, diarrhoea, } \\
\text { dysentery }\end{array}$ & & anticonvulsant & $\begin{array}{l}\text { Cameroon, } \\
\text { Central Africa } \\
\text { West Africa, } \\
\text { South Africa }\end{array}$ \\
\hline Bidens pilosa & Leaves & Decoction & $\begin{array}{l}\text { Dizziness, migraines, } \\
\text { headaches, rheumatism }\end{array}$ & & $\begin{array}{l}\text { Anti } \\
\text { hypertensive }\end{array}$ & $\begin{array}{l}\text { Cameroon, } \\
\text { Central } \\
\text { America } \\
\end{array}$ \\
\hline $\begin{array}{l}\text { Bryophyllum } \\
\text { pinnatum }\end{array}$ & Leaves & \begin{tabular}{|l|} 
Applica- \\
tion on \\
head \\
Decoction
\end{tabular} & $\begin{array}{l}\text { Convulsions, rheumatism } \\
\text { Arthritis }\end{array}$ & $\begin{array}{l}\text { Flavonoids, } \\
\text { antraquinones }\end{array}$ & \begin{tabular}{|l|} 
Antinociceptive \\
, anti- \\
inflammatory \\
antidiabetic \\
\end{tabular} & Central Africa \\
\hline Citrus sinenis & $\begin{array}{l}\text { Leaves + } \\
\text { Flowers } \\
\text { Barks } \\
\text { Roots }\end{array}$ & \begin{tabular}{|l|} 
Decoction \\
Infusion
\end{tabular} & $\begin{array}{l}\text { Epilepsy, convulsions, } \\
\text { Insomnia, agitation } \\
\text { Headaches, Malaria fever } \\
\text { Anxiety, schizophrenia }\end{array}$ & & Sedative & $\begin{array}{l}\text { Humid } \\
\text { tropical areas }\end{array}$ \\
\hline $\begin{array}{l}\text { Clerodendron } \\
\text { thomsoniae }\end{array}$ & $\begin{array}{l}\text { Leaves } \\
\text { Roots }\end{array}$ & Decoction & $\begin{array}{l}\text { Convulsions, head aches } \\
\text { Parasitic diseases }\end{array}$ & & \begin{tabular}{|l|} 
effect on \\
purinergic \\
neurotransmissi \\
on
\end{tabular} & $\begin{array}{l}\text { Cameroon, } \\
\text { India }\end{array}$ \\
\hline $\begin{array}{l}\text { Daniellia } \\
\text { oliveri }\end{array}$ & \begin{tabular}{|l} 
Barks \\
Roots
\end{tabular} & & $\begin{array}{l}\text { Epilepsy, Migraine, head } \\
\text { aches } \\
\text { Epilepsy, anxiety, } \\
\text { schizophrenia }\end{array}$ & & & $\begin{array}{l}\text { Angola, } \\
\text { Cameroon } \\
\text { Sudan, West } \\
\text { Africa Central } \\
\text { Africa } \\
\end{array}$ \\
\hline \begin{tabular}{|l|} 
Datura \\
stramonium
\end{tabular} & $\begin{array}{l}\text { Fruits } \\
\text { Leaves }\end{array}$ & & $\begin{array}{l}\text { Epilepsy } \\
\text { Coughs, asthma, pains }\end{array}$ & $\begin{array}{l}\text { Alkaloids, } \\
\text { atropine }\end{array}$ & & $\begin{array}{l}\text { Africa, Asia, } \\
\text { America, } \\
\text { Europa } \\
\end{array}$ \\
\hline \begin{tabular}{|l|} 
Detarium \\
microcarpum
\end{tabular} & $\begin{array}{l}\text { Leaves } \\
\text { Barks } \\
\text { Roots }\end{array}$ & Decoction & $\begin{array}{l}\text { Dizziness, schizophrenia, } \\
\text { paralysis malaria, diarrhoea } \\
\text { Epilepsy, Pains } \\
\text { Paralysis }\end{array}$ & & & $\begin{array}{l}\text { West Africa } \\
\text { Central Africa }\end{array}$ \\
\hline $\begin{array}{l}\text { Euphorbia } \\
\text { hirta }\end{array}$ & Whole plant & Decoction & $\begin{array}{l}\text { Convulsions, Insomnia } \\
\text { Diarrhoea, amoeba, asthma, } \\
\text { coughs, pains }\end{array}$ & $\begin{array}{l}\text { Alkaloids, } \\
\text { tannins }\end{array}$ & Anxiolytic & $\begin{array}{l}\text { Africa } \\
\text { continent }\end{array}$ \\
\hline $\begin{array}{l}\text { Flacourtia } \\
\text { indica }\end{array}$ & $\begin{array}{l}\text { Sterm barks } \\
\text { Fruts } \\
\text { Leaves }\end{array}$ & & $\begin{array}{l}\text { Epilepsy, headache, fever, } \\
\text { stomach-ache, diarrhoea } \\
\text { Sleep disorders }\end{array}$ & $\begin{array}{l}\text { beta-sistosterol } \\
\text { butyrolactone, } \\
\text { steroids, } \\
\text { flacourtine, } \\
\text { flavonoids, } \\
\text { coumarine, } \\
\text { terpenoids, } \\
\text { polyphenols }\end{array}$ & \begin{tabular}{|l|} 
Antiplasmodial \\
Protection \\
against liver \\
toxicity
\end{tabular} & \\
\hline
\end{tabular}




\begin{tabular}{|c|c|c|c|c|c|c|}
\hline $\begin{array}{c}\text { Name of the } \\
\text { plant }\end{array}$ & $\begin{array}{l}\text { Part of the } \\
\text { plant used }\end{array}$ & \begin{tabular}{|c|}
$\begin{array}{c}\text { Form of } \\
\text { the } \\
\text { medicine }\end{array}$ \\
\end{tabular} & Diseases & $\begin{array}{c}\text { Chemical } \\
\text { characterization }\end{array}$ & $\begin{array}{l}\text { Pharmaco- } \\
\text { logical } \\
\text { properties }\end{array}$ & Country \\
\hline $\begin{array}{l}\text { Hymenocardia } \\
\text { acida }\end{array}$ & $\begin{array}{l}\text { Leaves } \\
\text { Barks } \\
\text { Roots }\end{array}$ & $\begin{array}{l}\text { Infusion } \\
\text { Powder }\end{array}$ & $\begin{array}{l}\text { Headaches, fever, } \\
\text { hypotension, diabetes, sickle } \\
\text { cells } \\
\text { Epilepsy, schizophrenia }\end{array}$ & & & \begin{tabular}{|l|} 
Cameroon, \\
Central Africa \\
West Africa
\end{tabular} \\
\hline \begin{tabular}{|l|} 
Jatropha \\
gossypiifolia
\end{tabular} & \begin{tabular}{|l|} 
Leaves + \\
Roots \\
Roots
\end{tabular} & & $\begin{array}{l}\text { Convulsions, fever, } \\
\text { hypertension } \\
\text { Convulsions, }\end{array}$ & & & \begin{tabular}{|l|} 
Cameroon, \\
Central Africa, \\
West Africa
\end{tabular} \\
\hline \begin{tabular}{|l|} 
Khaya \\
senegalensis
\end{tabular} & $\begin{array}{l}\text { Leaves, Barks } \\
\text { Roots }\end{array}$ & Decoction & $\begin{array}{l}\text { Headaches, schizophrenia, } \\
\text { malaria } \\
\text { Fever }\end{array}$ & \begin{tabular}{|l|} 
Saponins, \\
tannins, \\
triterpenes
\end{tabular} & $\begin{array}{l}\text { Antiinflamm- } \\
\text { atory }\end{array}$ & Cameroon \\
\hline $\begin{array}{l}\text { Mentha } \\
\text { cordifolia }\end{array}$ & Leaves & Infusion & Insomnia, muscle relaxant, & & Antioxydant & Cameroon \\
\hline \begin{tabular}{|l|} 
Prosopis \\
Africana
\end{tabular} & $\begin{array}{l}\text { Leaves } \\
\text { Barks }\end{array}$ & Decoction & \begin{tabular}{|l|} 
Epilepsy, insomnia, anxiety \\
states, headaches, migraine, \\
agitation, fever \\
Vermifuge, fever
\end{tabular} & & $\begin{array}{l}\text { Antitrypano- } \\
\text { somal }\end{array}$ & $\begin{array}{l}\text { Cameroon } \\
\text { West Africa }\end{array}$ \\
\hline \begin{tabular}{|l|} 
Ricinus \\
communis
\end{tabular} & \begin{tabular}{|l|}
$\begin{array}{l}\text { Leaves }+ \\
\text { flowers }\end{array}$ \\
\end{tabular} & Decoction & \begin{tabular}{|l|}
$\begin{array}{l}\text { Epilepsy, convulsions, } \\
\text { headaches, diarrhea, asthma }\end{array}$ \\
\end{tabular} & ricin & \begin{tabular}{|l|}
$\begin{array}{l}\text { Neuroleptic like } \\
\text { properties }\end{array}$ \\
\end{tabular} & \begin{tabular}{|l|} 
Central Africa, \\
West Africa \\
\end{tabular} \\
\hline \begin{tabular}{|l|} 
Securidaca \\
longepeduncul \\
ata
\end{tabular} & $\begin{array}{l}\text { Barks } \\
\text { Roots, Leaves }\end{array}$ & & $\begin{array}{l}\text { Epilepsy, schizophrenia } \\
\text { Pains, Rheumatisms }\end{array}$ & & Anxiolytic & $\begin{array}{l}\text { Central Africa, } \\
\text { West Africa }\end{array}$ \\
\hline \begin{tabular}{|l|} 
Senna \\
singueana
\end{tabular} & $\begin{array}{l}\text { Leaves } \\
\text { Leaves and } \\
\text { flowers } \\
\text { Barks and } \\
\text { Roots, }\end{array}$ & & $\begin{array}{l}\text { Fever, } \\
\text { Conjunctivitis, } \\
\text { Convulsions, gonorrhoea, } \\
\text { bilharzias, stomach-aches, } \\
\text { constipation, } \\
\text { Epilepsy, syphilis, } \\
\end{array}$ & $\begin{array}{l}\text { 7-Methylphyscion } \\
\text { Cassiamin A }\end{array}$ & & \begin{tabular}{|l|} 
Cameroon, \\
Mali, Soudan, \\
East and \\
South Africa.
\end{tabular} \\
\hline \begin{tabular}{|l|} 
Terminalia \\
glaucescens,
\end{tabular} & $\begin{array}{l}\text { Leaves } \\
\text { Barks } \\
\text { Roots }\end{array}$ & $\begin{array}{l}\text { Decoction } \\
\text { Decoction } \\
\text { Macera- } \\
\text { tion }\end{array}$ & $\begin{array}{l}\text { Malaria, stomach-aches, } \\
\text { leucorrhoea, Hepatitis, } \\
\text { leucorrhoea } \\
\text { Epilepsy, diarrhoea, } \\
\text { leucorrhoea }\end{array}$ & $\begin{array}{l}\text { Terminalin A } \\
\text { Glaucinoic Acid }\end{array}$ & antimicrobial & \\
\hline \begin{tabular}{|l|}
$\begin{array}{l}\text { Terminalia } \\
\text { mollis }\end{array}$ \\
\end{tabular} & Roots & & Epilepsy & & & Central Africa, \\
\hline $\begin{array}{l}\text { Tetrapleura } \\
\text { tétraptera }\end{array}$ & $\begin{array}{l}\text { Barks } \\
\text { Fruits } \\
\text { Roots }\end{array}$ & Decoction & $\begin{array}{l}\text { Epilepsy } \\
\text { Convulsions } \\
\text { Fevers, malaria }\end{array}$ & Saponins, tannins & Anticonvulsant & \begin{tabular}{|l|} 
Angola, \\
Cameroon, \\
Sudan, West \\
Africa, Central \\
Africa \\
\end{tabular} \\
\hline \begin{tabular}{|l|} 
Trichilia \\
emetica
\end{tabular} & $\begin{array}{l}\text { Roots } \\
\text { Barks }\end{array}$ & & $\begin{array}{l}\text { Epilepsy, anti-parasitic } \\
\text { diseases } \\
\text { Head aches }\end{array}$ & Tannins, sterols & & $\begin{array}{l}\text { Savannah belt, } \\
\text { open } \\
\text { woodland in } \\
\text { Africa } \\
\end{array}$ \\
\hline $\begin{array}{l}\text { Vitelaria } \\
\text { paradoxa }\end{array}$ & $\begin{array}{l}\text { Leaves } \\
\text { Leaves }+ \\
\text { Barks }\end{array}$ & Decoction & $\begin{array}{l}\text { Convulsions, Epilepsy, } \\
\text { headaches, stress } \\
\text { Head aches }\end{array}$ & $\begin{array}{l}\text { Saponins, } \\
\text { alkaloids, } \\
\text { tannins, cadiac } \\
\text { glycosides } \\
\end{array}$ & Antimicrobial & $\begin{array}{l}\text { Cameroon, } \\
\text { Brazil }\end{array}$ \\
\hline
\end{tabular}

Table 1. Parts of the plant, form of the medicine and diseases treated in traditional medicine. Adeyemi et al., 2010; Adjanohoum et al., 1984; Adjanouhoun et al., 1996; Adzu et al., 2003; Agassounon et al. 2008; Anete et al., 1998 ; Anuradha et al., 2008; Arbonnier, 2000; Berhaut, 1975; Brenan, 1959 ; Dimo et al., 2002; El-Mahmood et al., 2008; Ezugwu \& Odoh, 2003; Gusman-Gutierez \& Navarrete, 2009; Iwu, 1993; Joyner, 2004; Lompo et al., 1998; Malgras, 1992; Mutasa et al., 1990; Nazneen et al., 2009; Ogundiya, 2009; Ojewole, 2005; Palgrave, 2003; Pathak et al., 2010; Pousset, 1989; Satyarayana et al., 1996; Sunday et al., 2009; Saulnier, 1998; Seema Zareen, 2006; Worapan et al., 2009. 
with these plants, very few were done to study their sedative and anticonvulsant properties. This study was undertaken to evaluate the anticonvulsant and sedative properties of these plants used in the treatment of insomnia and epilepsy in traditional medicine in Africa, particularly in Cameroon.

\section{Materials and methods}

\subsection{Animals}

Adult male mice (Mus musculus Swiss; $22 \pm 2$ g; 6 or 8 per group) were used for this study. The animals were housed in standard cages at $25^{\circ} \mathrm{C}$, on a $12 / 12 \mathrm{~h}$ light-dark cycle. They were supplied with food and water ad libitum.

Drugs were administered in a volume of $10 \mathrm{ml} / \mathrm{kg}$ of mice body weight. The study was conducted in accordance with the nationally ( $\mathrm{N}^{\circ}$.FWA-IRB00001954) and internationally accepted principles for laboratory animal use and care. In diazepam or sodium thiopentalinduced sleep tests, mice were divided into negative control group that received distilled water and four test groups that received different doses of the plant extracts. In anticonvulsant tests, there was one more group that received a known anticonvulsant compound and served as a positive control.

\subsection{Plant material}

A voucher specimen of each plant was authenticated by a botanist, Professor Mapongmetsem Pierre Marie, Department of Biological Sciences, University of Ngaoundéré and deposited at the National Herbarium of Cameroon in Yaoundé.

\subsection{Preparation of the extracts}

\subsubsection{Decoction}

$10 \mathrm{~g}$ of each plant material were macerated for $1 \mathrm{~h}$ in an amount of distilled water $(25,50$, 75, 100 or $150 \mathrm{ml}$ ) according to the plant. The mixture was boiled for $20 \mathrm{~min}$. After cooling, the supernatant (decoction) was collected and filtered. The decoction of each plant was diluted in distilled water to obtain less concentrated solutions. In another experiment, the decoction was dried and the $\mathrm{w} / \mathrm{w}$ yield of the extract was calculated (table 2). The decoctions were prepared according to the methods close to the ones used in traditional medicine.

\subsubsection{Maceration}

$10 \mathrm{~g}$ of dried fruits of Datura stramonium were macerated in $50 \mathrm{ml}$ of distilled water. After 1 $h$ the supernatant was collected, filtered and used in mice. The $w / w$ yield of the extract was obtained (table 2).

\subsection{Anticonvulsant tests}

\subsubsection{N-methyl-D-aspartate (NMDA) test}

Six groups of 6 or 8 mice received different treatments. Group I (negative control) was treated with distilled water. Groups II to V (test groups) were treated with 4 doses of the plant extracts. Group VI (positive control) was treated with $3 \mathrm{mg} / \mathrm{kg}$ of CGP 37849 i.p. or $33 \mathrm{\eta mol} / \mathrm{kg}$ of D-AP7 i.p. Mice were injected subcutaneously with NMDA, $75 \mathrm{mg} / \mathrm{kg} 1 \mathrm{~h}$ after administration of the different treatments. They were observed for $30 \mathrm{~min}$. Animals 
that did not exhibit turning behaviour within the $30 \mathrm{~min}$ of observation were declared protected. Turning behaviour was characterised by two consecutive $360^{\circ}$ cycles fulfilled by the same animal (Croucher et al., 1982; Ngo Bum et al., 2001; 2009a; 2009b; Schmutz et al., 1990).

\subsubsection{Strychnine (STR) test}

Six groups of 6 or 8 mice received different treatments as above, except that group VI (positive control) was treated with clonazepam $(3 \mathrm{mg} / \mathrm{kg}$, i.p.). Convulsions followed by death were induced in mice by the i.p. injection of $2.5 \mathrm{mg} / \mathrm{kg}$ STR nitrate $1 \mathrm{~h}$ after administration of the different treatments. The animals which survived more than $10 \mathrm{~min}$ after strychnine injection were qualified protected (Ngo Bum et al., 2001, 2009a).

\subsubsection{Picrotoxine (PIC) test}

Six groups of 6 or 8 mice received different treatments as above, except that group VI (positive control) was treated with clonazepam $(0.4 \mathrm{mg} / \mathrm{kg}$, i.p.). Clonic seizures were induced in mice by the i.p. injection of $7.5 \mathrm{mg} / \mathrm{kg}$ PIC $1 \mathrm{~h}$ after administration of the different treatments. The animals which did not convulse within the $15 \mathrm{~min}$ of observation after PIC injection were qualified protected (Lehmann et al., 1988; Ngo Bum et al., 2001).

\subsubsection{Pentylenetetrazol (PTZ) test}

Six groups of 6 or 8 mice received different treatments as above, except that group VI (positive control) was treated with clonazepam $(0.1 \mathrm{mg} / \mathrm{kg}$, i.p.). Clonic seizures were induced in mice by the i.p. injection of $70 \mathrm{mg} / \mathrm{kg}$ PTZ $1 \mathrm{~h}$ after administration of the different treatments. The animals that did not convulse within the $10 \mathrm{~min}$ from the injection of PTZ were qualified protected (Ngo Bum et al., 2001, 2009a, 2009b).

\subsubsection{Isonicotinic hydrazide acid (INH) test}

Six groups of 6 or 8 mice received different treatments as above, except that group VI (positive control) was treated with diazepam, $10 \mathrm{mg} / \mathrm{kg}$ (per os). Animals were injected i.p. with INH $250 \mathrm{mg} / \mathrm{kg} 1 \mathrm{~h}$ after the administration of the different treatments. The time to the onset of clonic or tonic seizures was recorded. (Bernasconi et al., 1988; Ngo Bum et al., 2001).

\subsection{Diazepam or sodium thiopental-induced sleep in mice}

Five groups of 6 or 8 mice received different treatments. Group I (negative control) was treated with distilled water and groups II to V (test groups) were treated with 4 doses of the plant extracts.The methods described by Beretz et al., (1978) and modified by Rakotonirina et al., (2001) were used. Sleep potentiating effects of the plant were studied in mice that received sodium thiopental or diazepam at a dose of $50 \mathrm{mg} / \mathrm{kg}$ (i.p.) 1 hour after the administration of the different treatments. The time between the loss of the straightening reflex and the regain of this reflex measured the sleeping time. The loss or the regain of the straightening reflex was measured by stimulating the external ear. When the mouse anterior paw does not move after stimulation with horsehair, the animal is sleeping. When the mouse is awakened, it moves and shakes its paw. 


\begin{tabular}{|c|c|c|c|c|c|}
\hline Name of the plant & $\begin{array}{l}\text { Part of the } \\
\text { plant used }\end{array}$ & \begin{tabular}{|c|} 
Quantity \\
plant powder \\
(g)
\end{tabular} & $\begin{array}{l}\text { Quantity of } \\
\text { water }(\mathrm{ml})\end{array}$ & $\begin{array}{l}\text { Yield } \\
(\%)\end{array}$ & $\begin{array}{c}\text { Root of } \\
\text { administra- } \\
\text { tion } \\
\end{array}$ \\
\hline Annona muricata & Fresh leaves & 10 & 50 & 6 & i.p. \\
\hline Annona senegalensis & Dried leaves & 10 & 75 & 5 & i.p. \\
\hline Bidens pilosa & Fresh leaves & 10 & 25 & 3.5 & i.p. \\
\hline Bryophyllum pinnatum & Fresh leaves & 10 & 25 & 7 & i.p. \\
\hline Citrus sinenis & Fresh leaves & 10 & 50 & 5 & i.p. \\
\hline Clerodendron thomsoniae & Dried leaves & 10 & 50 & 6.7 & i.p. \\
\hline Daniellia oliveri & Dried barks & 10 & 50 & 9.9 & p.o. \\
\hline Datura stramonium & Dried fruits & 10 (macerate) & 50 & 7 & i.p. \\
\hline Detarium microcarpum & Dried roots & 10 & 50 & 7.43 & p.o. \\
\hline Euphorbia hirta & Fresh plant & 10 & 50 & 7 & i.p. \\
\hline Flacourtia indica & Dried barks & 10 & 100 & 10 & p.o. \\
\hline Hymenocardia acida & Fresh leaves & 10 & 25 & 2.19 & i.p. \\
\hline Jatropha gossypiifolia & Dried leaves & 10 & 50 & 7 & i.p. \\
\hline Khaya senegalensis & Dried leaves & 10 & 75 & 5 & i.p. \\
\hline Mentha cordifolia & Fresh leaves & 10 & 50 & 7 & i.p. \\
\hline Prosopis Africana & Dried leaves & 10 & 50 & 5.6 & i.p. \\
\hline Ricinus communis & Fresh leaves & 10 & 50 & 6 & p.o. \\
\hline $\begin{array}{l}\text { Securidaca } \\
\text { longepedunculata }\end{array}$ & Dried roots & 10 & 150 & 10 & i.p. \\
\hline Senna singueana & Dried roots & 10 & 50 & 8 & p.o. \\
\hline Terminalia glaucescens & Dried roots & 10 & 100 & 7.6 & p.o. \\
\hline Terminalia mollis & Dried roots & 10 & 50 & 7.1 & p.o. \\
\hline Tetrapleura tétraptera & Dried barks & 10 & 50 & 4.2 & i.p. \\
\hline Trichilia emetic & Fresh roots & 10 & 50 & 6.3 & p.o. \\
\hline Vitelaria paradoxa & Fresh leaves & 10 & 150 & 12.6 & i.p. \\
\hline
\end{tabular}

i.p. (intraperitoneal), p.o. (per os).

Table 2. Quantities of plants powder and distilled water, and part of the plant used to prepare the decoctions.

\subsection{Statistical analysis}

Three parameters were measured: the protection against chemically-induced seizures, the latency to the onset of seizures (min) in INH test, the latency to the onset of sleep and the sleeping time ( $\mathrm{min}$ ) in the sleep potentiation test. Data of the control groups were compared to data of groups treated with the plants extracts and to data of the positive control groups. The statistical analysis were done using Fisher exact test and Anova followed by Dunnett (REGWQ). P $<0.05$ was considered significant.

\subsection{Chemicals}

D-2-amino-7-phosphonoheptanoate, Clonazepam, Isonicotinic hydrazide acid, N-methyl-Daspartate, penthylenetetrazol, picrotoxine, sodium thiopental and strychnine are from Sigma Chemical, USA. Diazepam is from Roche, France. 


\section{Results}

\subsection{Sedative properties}

The extracts of twenty one plants increased in a dose-dependent manner the sleeping time induced by sodium thiopental or diazepam. The most potent was Datura stramonium. it multiplied by a factor of 5 the sleeping time of the control group (from $16 \pm 7$ to $94 \pm 25 \mathrm{~min}$ at a dose of $70 \mathrm{mg} / \mathrm{kg}$ ), but this extract was very toxic for animals. The decoctions of eight plants multiplied by a factor of 4 the sleeping time of their control group: Annona senegalensis (from $19 \pm 4$ to $89 \pm 29 \mathrm{~min}$ at a dose of $67 \mathrm{mg} / \mathrm{kg}$ ), Clerodendron thomsoniae (from $19 \pm 3$ to $94 \pm 30 \mathrm{~min}$ at a dose of $134 \mathrm{mg} / \mathrm{kg}$ ), Daniellia oliveri (from $20 \pm 8$ to $81 \pm 13 \mathrm{~min}$ at a dose of $198 \mathrm{mg} / \mathrm{kg}$ ), Hymenocardia acida (from $20 \pm 11$ to $85 \pm 21 \mathrm{~min}$ at a dose of 87.6 $\mathrm{mg} / \mathrm{kg}$ ), Securidaca longepedunculata (from $18 \pm 3$ to $78 \pm 14 \mathrm{~min}$ at a dose of $66.7 \mathrm{mg} / \mathrm{kg}$ ), Terminalia mollis (from $17 \pm 1$ to $84 \pm 15 \mathrm{~min}$ at a dose of $70 \mathrm{mg} / \mathrm{kg}$ ), Tetrapleura tetraptera (from $19 \pm 3$ to $91 \pm 15 \mathrm{~min}$ at a dose of $84 \mathrm{mg} / \mathrm{kg}$ ) and Trichilia emetica (from $17 \pm 1$ to $84 \pm 10$ $\min$ at a dose of $126 \mathrm{mg} / \mathrm{kg}$ ). The sleeping time of the control groups were multiplied by a factor of 3 by six plants: Flacourtia indica (from $16 \pm 12$ to $49 \pm 3 \mathrm{~min}$ at a dose of $100 \mathrm{mg} / \mathrm{kg}$ ), Jatropha gossypiifolia (from $11 \pm 5$ to $43 \pm 15 \mathrm{~min}$ at a dose of $140 \mathrm{mg} / \mathrm{kg}$ ), Prosopis Africana (from $19 \pm 3$ to $61 \pm 26 \mathrm{~min}$ at a dose of $112 \mathrm{mg} / \mathrm{kg}$ ), Senna singueana (from $24 \pm 2$ to $86 \pm 5$ $\mathrm{min}$ at a dose of $20 \mathrm{mg} / \mathrm{kg}$ ), Terminalia glaucescens (from $37 \pm 13$ to $120 \pm 21 \mathrm{~min}$ at a dose of $76 \mathrm{mg} / \mathrm{kg}$ ), and Vitellaria paradoxa (from $25 \pm 4$ to $84 \pm 20 \mathrm{~min}$ at a dose of $84 \mathrm{mg} / \mathrm{kg}$ ). The decoctions of five plants multiplied by a factor of 2 the sleeping time of their control group: Annona muricata (from $31 \pm 11$ to $71 \pm 15 \mathrm{~min}$ at a dose of $120 \mathrm{mg} / \mathrm{kg}$ ), Bidens pilosa (from 31 \pm 2 to $80 \pm 2 \mathrm{~min}$ at a dose of $140 \mathrm{mg} / \mathrm{kg}$ ), Detarium microcarpum (from $20 \pm 6$ to $52 \pm 12 \mathrm{~min}$ at a dose of $111.45 \mathrm{mg} / \mathrm{kg}$ ), Euphorbia hirta (from $56 \pm 16$ to $145 \pm 10 \mathrm{~min}$ at a dose of 140 $\mathrm{mg} / \mathrm{kg}$ ) and Mentha cordifolia (from $10 \pm 2$ to $24 \pm 3 \mathrm{~min}$ at a dose of $140 \mathrm{mg} / \mathrm{kg}$ ). Bryophyllum pinnatum induced a slight increase of the sleeping time. Only Citrus sinenis and Kaya senegalensis could not increase the total sleep time of mice (table 3). Some of those plants also reduced the onset time of sleep (Table 4 ).

\subsection{Anticonvulsant properties}

\subsubsection{On PTZ- induced convulsions}

$78.3 \%$ of plants extract were effective against PTZ-induced convulsions. Annona muricata, Annona senegalensis, Bidens pilosa, Clerodendron thomsoniae, Daniellia oliveri, Datura stramonium, Detarium microcarpum, Euphorbia hirta, Flacourtia indica, Hymenocardia acida, Mentha cordifolia, Ricinus communis, Securidaca longepedunculata, Senna singueana, Terminalia glaucescens, Terminalia mollis, Tetrapleura tétraptera, Trichilia emetica and Vitellaria paradoxa protected mice against convulsions induced by PTZ (table 5).

\subsubsection{On STR- induced convulsions}

The percentage of plants extracts that protected mice against STR-induced convulsions was 77.8\%. Annona muricata, Bidens pilosa, Daniellia oliveri, Detarium microcarpum, Flacourtia indica, Hymenocardia acida, Jatropha gossypiifolia, Khaya senegalensis, Mentha cordifolia, Prosopis Africana, Securidaca longepedunculata, Senna singueana, Terminalia mollis, Trichilia emetica protected mice against STR- induced convulsions (table 5).

\subsubsection{On PIC- induced convulsions}

The percentage of plants extracts that protected mice against PIC-induced convulsions was 87.5\%. Clerodendron thomsoniae, Flacourtia indica, Mentha cordifolia, Securidaca longepedunculata, 
Senna singueana, Terminalia glaucescens and Vitellaria paradoxa protected mice against convulsions induced by PIC (table 5).

\begin{tabular}{|c|c|c|c|c|c|c|}
\hline & & & & Doses o & nts in $\mathrm{mg}$ & \\
\hline Daniellia & & $\mathrm{CON}$ & 49.5 & 99 & 148.5 & 198 \\
\hline oliveri & DIAZ & $9 \pm 3$ & $6 \pm 1$ & $6 \pm 1$ & $5 \pm 2$ & $3 \pm 1^{* *}$ \\
\hline Detarium & & $\mathrm{CON}$ & 37.15 & 47.3 & 111.45 & 148.6 \\
\hline microcarpum & DIAZ & $9 \pm 3$ & $7 \pm 2$ & $6 \pm 3$ & $4 \pm 2^{*}$ & $6 \pm 3$ \\
\hline Flacourtia & & CON & 10 & 25 & 50 & 100 \\
\hline indica & DIAZ & $4 \pm 2$ & $8 \pm 6$ & $4 \pm 4$ & $11 \pm 6$ & $4 \pm 4$ \\
\hline Hymenocardia & & $\mathrm{CON}$ & 8.7 & 21.9 & 43.8 & 87.6 \\
\hline acida & DIAZ & $9 \pm 3$ & $7 \pm 3$ & $6 \pm 1^{*}$ & $5 \pm 1^{* *}$ & $3 \pm 1^{* * *}$ \\
\hline Mentha & & $\mathrm{CON}$ & 14 & 35 & 70 & 140 \\
\hline cordifolia & DIAZ & $3 \pm 1$ & $2 \pm 1$ & $2 \pm 1$ & $6 \pm 3$ & $4 \pm 1$ \\
\hline Securidaca & & $\mathrm{CON}$ & 10 & 20 & 50 & 66.7 \\
\hline longepedunculata & DIAZ & $6 \pm 1$ & $5 \pm 1$ & $5 \pm 1$ & $4 \pm 1^{*}$ & $4 \pm 1^{*}$ \\
\hline Senna & & CON & 20 & 40 & 80 & 160 \\
\hline singueana & DIAZ & $15 \pm 3$ & $6 \pm 1^{* * *}$ & $6 \pm 1^{* * *}$ & $7 \pm 1^{* * *}$ & $8 \pm 1^{* * *}$ \\
\hline Terminalia & & CON & 9.5 & 19 & 38 & 76 \\
\hline glaucescens & DIAZ & $4 \pm 2$ & $7 \pm 4$ & $5 \pm 1$ & $6 \pm 3$ & $2 \pm 2$ \\
\hline Terminalia & & $\mathrm{CON}$ & 14 & 35 & 70 & 140 \\
\hline mollis & DIAZ & $7 \pm 2$ & $5 \pm 1$ & $2 \pm 1^{* *}$ & $3 \pm 1^{* *}$ & $4 \pm 1^{*}$ \\
\hline Trichilia & & CON & 12.6 & 33 & 66 & 126 \\
\hline emetica & DIAZ & $6 \pm 1$ & $5 \pm 1$ & $4 \pm 1^{* * *}$ & $2 \pm 1^{* * *}$ & $2 \pm 1^{* * *}$ \\
\hline
\end{tabular}

Data represent the onset time of sleep time. Values are means \pm ESM. $N=6$ or 8 per dose, ${ }^{*}<\mathrm{p} 0.05,{ }^{* *}<\mathrm{p}$ $0.01{ }^{* * *}<\mathrm{p} 0.001$ vs control, Anova followed by Dunnett (REGWQ). CON = distilled water, DIAZ = diazepam $50 \mathrm{mg} / \mathrm{kg}$.

Table 3. The effects of the different plants on the onset time of sleep induced in mice by sodium thiopental or diazepam.

\subsubsection{On NMDA- induced turning behaviour}

The percentage of plants extracts that protected mice against NMDA-induced turning behaviour was 100\%. Annona muricata, Bidens pilosa, Bryophyllum pinnatum, Citrus sinenis, Euphorbia hirta, Khaya senegalensis protected mice against turning behaviour induced by NMDA (table 5).

\subsubsection{On MES- induced convulsions}

The percentage of plants extracts that protected mice against MES-induced convulsions was $25 \%$. Securidaca longepedunculata protected mice against convulsions induced by MES (table 5).

\subsubsection{On INH- induced convulsions}

The percentage of plants extracts that were effective against INH-induced convulsions in mice was $60 \%$. Ricinus communis, Securidaca longepedunculata, Senna singueana delayed the onset of seizures in INH test (table 5).

\subsubsection{Plants efficacy}

Flacourtia indica, Ricinus communis, Securidaca longepedunculata, Senna singueana, Terminalia glaucescens showed very good anticonvulsant activities (80 to $100 \%$ of protection against PTZ, PIC or INH induced seizures). The other eighteen plants tested protected 50 to $75 \%$ of 
Doses of the plants in $\mathrm{mg} / \mathrm{kg}$

\begin{tabular}{|c|c|c|c|c|c|c|}
\hline $\begin{array}{l}\text { Annona } \\
\text { muricata }\end{array}$ & DIAZ & $\begin{array}{l}\text { CON } \\
31 \pm 11\end{array}$ & $\begin{array}{l}12 \\
51 \pm 26^{*}\end{array}$ & $\begin{array}{l}30 \\
67 \pm 6^{* * *}\end{array}$ & $\begin{array}{l}60 \\
68 \pm 2^{* * *}\end{array}$ & $\begin{array}{l}120 \\
71 \pm 15^{\star \star \star}\end{array}$ \\
\hline Annona & & $\mathrm{CON}$ & 6.7 & 17 & 34 & 67 \\
\hline senegalensis & DIAZ & $19 \pm 4$ & $52 \pm 18^{* * *}$ & $72 \pm 17^{* * *}$ & $79 \pm 25^{\star * *}$ & $89 \pm 29^{* * *}$ \\
\hline Bidens & & CON & 14 & 35 & 70 & 140 \\
\hline pilosa & DIAZ & $31 \pm 2$ & $70 \pm 2^{* * *}$ & $66 \pm 3^{* * *}$ & $79 \pm 5^{\star * *}$ & $80 \pm 2^{* * *}$ \\
\hline Bryophyllum & & $\mathrm{CON}$ & 28 & 70 & 140 & 280 \\
\hline pinnatum & DIAZ & $21 \pm 2$ & $32 \pm 5^{* *}$ & $26 \pm 5$ & $35 \pm 1^{* *}$ & $31 \pm 6^{*}$ \\
\hline Citrus & & CON & 10 & 25 & 50 & 100 \\
\hline sinenis & DIAZ & $56 \pm 24$ & $50 \pm 20$ & $40 \pm 10$ & $45 \pm 12$ & $57 \pm 10$ \\
\hline Clerodendron & & CON & 13.4 & 33.5 & 67 & 134 \\
\hline thomsoniae & DIAZ & $19 \pm 3$ & $39 \pm 9^{* * *}$ & $74 \pm 22^{* * *}$ & $90 \pm 16^{* * *}$ & $94 \pm 30^{* * *}$ \\
\hline Daniellia & & $\mathrm{CON}$ & 49.5 & 99 & 148.5 & 198 \\
\hline oliveri & DIAZ & $20 \pm 8$ & $50 \pm 3^{* * *}$ & $74 \pm 8^{* * *}$ & $74 \pm 7^{* * *}$ & $81 \pm 13^{* * *}$ \\
\hline Datura & & $\mathrm{CON}$ & 3.5 & 7 & 35 & 70 \\
\hline stramonium & THIO & $16 \pm 7$ & $55 \pm 15^{* * *}$ & $63 \pm 21^{* * *}$ & $85 \pm 23^{* * *}$ & $94 \pm 25^{* * *}$ \\
\hline Detarium & & $\mathrm{CON}$ & 37.15 & 47.3 & 111.45 & 148.6 \\
\hline microcarpum & DIAZ & $20 \pm 6$ & $38 \pm 10^{* *}$ & $46 \pm 13^{* *}$ & $52 \pm 12^{* * *}$ & $45 \pm 9^{* * *}$ \\
\hline Euphorbia & & CON & 14 & 35 & 70 & 140 \\
\hline hirta & DIAZ & $56 \pm 16$ & $99 \pm 24^{* *}$ & $97 \pm 21^{* *}$ & $117 \pm 26^{* * *}$ & $145 \pm 10^{* * *}$ \\
\hline Flacourtia & & $\mathrm{CON}$ & 10 & 25 & 50 & 100 \\
\hline indica & DIAZ & $16 \pm 12$ & $15 \pm 11$ & $38 \pm 25^{*}$ & $44 \pm 4^{* * *}$ & $49 \pm 3^{* * *}$ \\
\hline Hymenocardia & & $\mathrm{CON}$ & 8.7 & 21.9 & 43.8 & 87.6 \\
\hline acida & DIAZ & $20 \pm 11$ & $51 \pm 12^{* * *}$ & $70 \pm 15^{\star * *}$ & $77 \pm 23^{* * *}$ & $85 \pm 21^{* * *}$ \\
\hline Jatropha & & $\mathrm{CON}$ & 14 & 35 & 70 & 140 \\
\hline gossypiifolia & DIAZ & $11 \pm 5$ & $29 \pm 14^{*}$ & $27 \pm 13^{*}$ & $32 \pm 10^{* * *}$ & $43 \pm 15^{\star * *}$ \\
\hline Kaya & & CON & 6.7 & 17 & 34 & 67 \\
\hline senegalensis & DIAZ & $63 \pm 15$ & $52 \pm 21$ & $58 \pm 25$ & $58 \pm 23$ & $61 \pm 23$ \\
\hline Mentha & & CON & 14 & 35 & 70 & 140 \\
\hline cordifolia & DIAZ & $10 \pm 2$ & $16 \pm 5^{*}$ & $21 \pm 5^{* *}$ & $21 \pm 4^{* *}$ & $24 \pm 3^{* *}$ \\
\hline Prosopis & & CON & 11.2 & 28 & 56 & 112 \\
\hline africana & DIAZ & $19 \pm 3$ & $52 \pm 26^{*}$ & $57 \pm 17^{* *}$ & $31 \pm 17$ & $61 \pm 26^{* * *}$ \\
\hline Securidaca & & $\mathrm{CON}$ & 10 & 20 & 50 & 66.7 \\
\hline longepedunculata & DIAZ & $18 \pm 3$ & $60 \pm 27^{* *}$ & $65 \pm 14^{* * *}$ & $67 \pm 27^{* *}$ & $78 \pm 14^{* * *}$ \\
\hline Senna & & CON & 20 & 40 & 80 & 160 \\
\hline singueana & DIAZ & $24 \pm 2$ & $86 \pm 5^{* *}$ & $77 \pm 3^{* * *}$ & $44 \pm 7^{* *}$ & $29 \pm 9^{* * *}$ \\
\hline Terminalia & & CON & 9.5 & 19 & 38 & 76 \\
\hline glaucescens & DIAZ & $37 \pm 13$ & $31 \pm 15$ & $79 \pm 40^{*}$ & $86 \pm 17^{* * *}$ & $120 \pm 21^{* * *}$ \\
\hline Terminalia & & $\mathrm{CON}$ & 14 & 35 & 70 & 140 \\
\hline mollis & DIAZ & $17 \pm 1$ & $44 \pm 7^{* * *}$ & $64 \pm 11^{* * *}$ & $84 \pm 15^{* * *}$ & $73 \pm 8^{* * *}$ \\
\hline Tetrapleura & & CON & 8.4 & 21 & 42 & 84 \\
\hline tétraptera & DIAZ & $19 \pm 3$ & $39 \pm 10^{* *}$ & $67 \pm 18^{* * *}$ & $82 \pm 14^{* * *}$ & $91 \pm 15^{\star * *}$ \\
\hline Trichilia & & $\mathrm{CON}$ & 12.6 & 33 & 66 & 126 \\
\hline emetica & DIAZ & $17 \pm 1$ & $22 \pm 2^{*}$ & $29 \pm 4^{* *}$ & $71 \pm 7^{* * *}$ & $84 \pm 10^{* * *}$ \\
\hline Vitelaria & & $\mathrm{CON}$ & 12 & 21 & 42 & 84 \\
\hline paradoxa & DIAZ & $25 \pm 4$ & $40 \pm 13^{* * *}$ & $57 \pm 6^{* * *}$ & $59 \pm 8^{* * *}$ & $84 \pm 20^{* * *}$ \\
\hline
\end{tabular}

Data represent the total sleep time. Values are means \pm ESM. $N=6$ or 8 per dose, ${ }^{*} \mathrm{p}<0.05,{ }^{* *} \mathrm{p}<0.01$, ${ }^{* * *} \mathrm{p}<0.001$ vs control, Anova followed by Dunnett (REGWQ). CON = distilled water, DIAZ = diazepam $50 \mathrm{mg} / \mathrm{kg}$, THIO = sodium thiopental $50 \mathrm{mg} / \mathrm{kg}$.

Table 4 . The effects of the different plants on the total sleep time induced in mice by sodium thiopental or diazepam. 


\begin{tabular}{|c|c|c|c|c|c|c|c|}
\hline & & & & Doses o & nts in mg & & \\
\hline Annona & & CON & 12 & 30 & 60 & 120 & $\mathrm{CP}$ \\
\hline Muricata & PTZ & 0 & 16 & $50^{*}$ & 33 & $50^{*}$ & $100^{* * *}$ \\
\hline & STR & 0 & 12 & 0 & 12 & $50^{*}$ & $100^{* * *}$ \\
\hline & NMDA & 0 & 16 & 16 & 33 & $50^{*}$ & $100^{\star * *}$ \\
\hline Annona & & $\mathrm{CON}$ & 6.7 & 17 & 34 & 67 & $\mathrm{CP}$ \\
\hline $\begin{array}{l}\text { Senegalensis } \\
100^{* * *}\end{array}$ & PTZ & 0 & 12 & 37 & $50^{*}$ & 25 & \\
\hline Bidens & & $\mathrm{CON}$ & 14 & 35 & 70 & 140 & $\mathrm{CP}$ \\
\hline pilosa & PTZ & 0 & 16 & $50^{*}$ & 33 & $50^{*}$ & $100^{* * *}$ \\
\hline & STR & 0 & 16 & $50^{*}$ & 40 & $50^{*}$ & $100^{* * *}$ \\
\hline & NMDA & 0 & 33 & 33 & $66^{* *}$ & $50^{*}$ & $100^{* * *}$ \\
\hline Bryophyllum & & $\mathrm{CON}$ & 28 & 70 & 140 & 280 & $\mathrm{CP}$ \\
\hline pinnatum & PTZ & 0 & 16 & 33 & 16 & 33 & $100^{* * *}$ \\
\hline & STR & 0 & 0 & 10 & 0 & 16 & 100 *** \\
\hline & NMDA & 0 & 33 & $50^{*}$ & $50^{*}$ & $50^{*}$ & $100^{* * *}$ \\
\hline Citrus & & CON & 10 & 25 & 50 & 100 & $\mathrm{CP}$ \\
\hline sinenis & PTZ & 0 & 25 & 25 & 12 & 0 & $100^{* * *}$ \\
\hline & STR & 0 & 0 & 0 & 12 & 12 & $100^{\star \star *}$ \\
\hline & NMDA & 0 & $50^{*}$ & $50^{*}$ & $75^{* *}$ & $75^{* *}$ & $100^{* * *}$ \\
\hline Clerodendron & & $\mathrm{CON}$ & 13.4 & 33.5 & 67 & 134 & $\mathrm{CP}$ \\
\hline Thomsoniae & PTZ & 0 & 12 & 37 & 37 & $62^{*}$ & $100^{* * *}$ \\
\hline & PIC & 0 & 0 & 25 & $50^{*}$ & $50^{*}$ & $100^{* * *}$ \\
\hline Daniellia & & $\mathrm{CON}$ & 49.5 & 99 & 148.5 & 198 & $\mathrm{CP}$ \\
\hline oliveri & PTZ & 0 & 25 & 37 & 37 & $50^{*}$ & $100^{* * *}$ \\
\hline & STR & 0 & 16 & $66^{* *}$ & $50^{*}$ & $50^{*}$ & $100 * * *$ \\
\hline Detarium & & $\mathrm{CON}$ & 37 & 47 & 111 & 148 & $\mathrm{CP}$ \\
\hline microcarpum & PTZ & 0 & $50^{*}$ & 37 & 0 & 0 & $100^{\star * *}$ \\
\hline & STR & 0 & $50^{*}$ & 33 & 33 & 16 & $100^{* * *}$ \\
\hline Euphorbia & & CON & 14 & 35 & 70 & 140 & $\mathrm{CP}$ \\
\hline hirta & PTZ & 0 & 0 & 25 & 0 & $50^{*}$ & $100^{* * *}$ \\
\hline & STR & 0 & 12 & 37 & 25 & 37 & $100^{* * *}$ \\
\hline & NMDA & 0 & 33 & 33 & $50^{*}$ & $50^{*}$ & $100^{* * *}$ \\
\hline Flacourtia & & CON & 10 & 25 & 50 & 100 & $\mathrm{CP}$ \\
\hline indica & PTZ & 0 & $80^{* *}$ & $60^{*}$ & 40 & 40 & $100^{* * *}$ \\
\hline & STR & 0 & 20 & 40 & 40 & $60^{*}$ & $100^{* * *}$ \\
\hline & PIC & 0 & 40 & $60^{*}$ & $60^{*}$ & $80^{* *}$ & $100^{* * *}$ \\
\hline & MES & 0 & 40 & 0 & 0 & 40 & 100 \\
\hline & INH & $36 \pm 7$ & $48 \pm 5$ & $36 \pm 10$ & $50 \pm 12$ & $49 \pm 6$ & $73 \pm 11^{* * *}$ \\
\hline Hymenocardia & & $\mathrm{CON}$ & 8.76 & 21.9 & 43.8 & 87.6 & $\mathrm{CP}$ \\
\hline acida & PTZ & 0 & 0 & 25 & 37 & $62^{*}$ & $100^{* * *}$ \\
\hline & STR & 0 & 16 & 33 & 33 & $50^{*}$ & $100^{* * *}$ \\
\hline Jatropha & & CON & 14 & 35 & 70 & 140 & $\mathrm{CP}$ \\
\hline gossypiifolia & PTZ & 0 & 0 & 25 & 37 & 0 & $100^{* * *}$ \\
\hline & STR & 0 & $50^{*}$ & $50^{*}$ & $62^{*}$ & 25 & $100^{* * *}$ \\
\hline & PIC & 0 & 0 & 0 & 0 & 0 & $100^{* * *}$ \\
\hline Khaya & & $\mathrm{CON}$ & 6.7 & 17 & 34 & 67 & $\mathrm{CP}$ \\
\hline senegalensis & PTZ & 0 & 12 & 12 & 0 & 25 & $100^{* * *}$ \\
\hline & STR & 0 & 25 & $50^{*}$ & 25 & $50^{*}$ & $100^{* * *}$ \\
\hline & NMDA & 0 & $62^{*}$ & 33 & 33 & $50^{*}$ & $100^{* * *}$ \\
\hline Mentha & & CON & 14 & 35 & 70 & 140 & $\mathrm{CP}$ \\
\hline cordifolia & PTZ & 0 & 16 & 33 & $66^{* *}$ & $50^{*}$ & $100^{* * *}$ \\
\hline & STR & 0 & 33 & 33 & 33 & $66^{* *}$ & $100^{* * *}$ \\
\hline & PIC & 0 & 16 & $50^{*}$ & 33 & $50^{*}$ & $100^{* * *}$ \\
\hline Prosopis & & $\mathrm{CON}$ & 11.2 & 28 & 56 & 112 & $\mathrm{CP}$ \\
\hline africana & PTZ & 0 & 0 & 0 & 25 & 37 & $100^{* * *}$ \\
\hline & STR & 0 & $62^{*}$ & 25 & $50^{*}$ & $50^{*}$ & $100^{* * *}$ \\
\hline Ricinus & & CON & 12 & 30 & 60 & 120 & $\mathrm{CP}$ \\
\hline communis & PTZ & 0 & 37 & $50^{*}$ & $62^{*}$ & $87^{* * *}$ & $100^{\star * *}$ \\
\hline
\end{tabular}




\begin{tabular}{|c|c|c|c|c|c|c|c|}
\hline & $\mathrm{INH}$ & $31 \pm 9$ & $33 \pm 6$ & $36 \pm 8$ & $40 \pm 7$ & $56 \pm 16^{*}$ & $77 \pm 11^{* *}$ \\
\hline Securidaca & & $\mathrm{CON}$ & 10 & 20 & 50 & 66.7 & $\mathrm{CP}$ \\
\hline \multirow{4}{*}{ longepedunculata } & PTZ & 0 & $67^{* *}$ & $67^{* *}$ & $83^{* * *}$ & $100^{* * *}$ & $100 * * *$ \\
\hline & STR & 0 & $50^{*}$ & $67^{* *}$ & $67^{* *}$ & $67^{* *}$ & $100^{* * *}$ \\
\hline & PIC & 0 & $67^{* *}$ & $100^{* * *}$ & $83^{* *}$ & $83^{* *}$ & $100^{* * *}$ \\
\hline & INH & $46 \pm 3$ & $51 \pm 5^{*}$ & $62 \pm 12^{* *}$ & $67 \pm 20^{* *}$ & \multicolumn{2}{|c|}{$78 \pm 21^{* *} 97 \pm 20^{* * *}$} \\
\hline Senna & & $\mathrm{CON}$ & 20 & 40 & 80 & 160 & $\mathrm{CP}$ \\
\hline \multirow{5}{*}{ singueana } & PTZ & 0 & $80^{* *}$ & $80^{* *}$ & 40 & 40 & $100^{* * *}$ \\
\hline & STR & 0 & $80^{* *}$ & $80^{* *}$ & 40 & 40 & $100^{* * *}$ \\
\hline & PIC & 0 & 20 & $60^{*}$ & 0 & 20 & $100^{* * *}$ \\
\hline & MES & 0 & 40 & 40 & 20 & 20 & $80^{* *}$ \\
\hline & $\mathrm{INH}$ & $21 \pm 1$ & $30 \pm 1^{* *}$ & $29 \pm 6$ & $32 \pm 9^{* *}$ & $37 \pm 1^{* *}$ & $42 \pm 6^{* *}$ \\
\hline Terminalia & & CON & 9.5 & 19 & 38 & 76 & $\mathrm{CP}$ \\
\hline \multirow{5}{*}{ glaucescens } & PTZ & 0 & 40 & $60^{*}$ & 40 & $100^{* * *}$ & $100^{* * *}$ \\
\hline & STR & 0 & 40 & 20 & 0 & 0 & $100^{* * *}$ \\
\hline & PIC & 0 & 20 & $60^{*}$ & 40 & 20 & $100^{* * *}$ \\
\hline & MES & 0 & 20 & 40 & 0 & 40 & $80^{* *}$ \\
\hline & INH & $36 \pm 7$ & $41 \pm 13$ & $18 \pm 5$ & $42 \pm 9$ & $47 \pm 13$ & $85 \pm 26^{* * *}$ \\
\hline \multirow{3}{*}{$\begin{array}{l}\text { Terminalia } \\
\text { mollis }\end{array}$} & & CON & 14 & 35 & 70 & 140 & $\mathrm{CP}$ \\
\hline & PTZ & 0 & $50^{*}$ & 37 & 25 & 37 & $100^{* * *}$ \\
\hline & STR & 0 & $66^{* *}$ & 33 & 33 & $50^{*}$ & $100^{* * *}$ \\
\hline \multirow{2}{*}{$\begin{array}{l}\text { Tetrapleura } \\
\text { tetraptera }\end{array}$} & & CON & 8.4 & 21 & 42 & 84 & $\mathrm{CP}$ \\
\hline & PTZ & 0 & 25 & $50^{*}$ & $50^{*}$ & $50^{*}$ & $100^{* * *}$ \\
\hline \multirow{3}{*}{$\begin{array}{l}\text { Trichilia } \\
\text { emetica }\end{array}$} & & CON & 12.6 & 33 & 66 & 126 & $\mathrm{CP}$ \\
\hline & PTZ & 0 & 25 & $50^{*}$ & $50^{*}$ & $50^{*}$ & $100^{* * *}$ \\
\hline & STR & 0 & 12 & 25 & $50^{*}$ & $50^{*}$ & $100^{* * *}$ \\
\hline \multirow{3}{*}{$\begin{array}{l}\text { Vitelaria } \\
\text { paradoxa }\end{array}$} & & CON & 12 & 21 & 42 & 84 & $\mathrm{CP}$ \\
\hline & PTZ & 0 & 12 & $50^{*}$ & $62^{*}$ & $50^{*}$ & $100^{\star \star *}$ \\
\hline & PIC & 0 & 0 & 12 & $50^{*}$ & 37 & $100^{* * *}$ \\
\hline
\end{tabular}

Data represent the percentage of protected mice in different tests. $\mathrm{N}=6$ or 8 per dose, ${ }^{*} \mathrm{p}<0.05$, ${ }^{* *} \mathrm{p}<0.01,{ }^{* * *} \mathrm{p}<0.001$ vs control, Anova followed by Dunnett (REGWQ). CON (negative control) = distilled water, $\mathrm{CP}$ (positive control) $=$ clonazepam $0.1 \mathrm{mg} / \mathrm{kg}$ for PTZ test, clonazepam $0.4 \mathrm{mg} / \mathrm{kg}$ for PIC test, clonazepam $3 \mathrm{mg} / \mathrm{kg}$ for STR test, diazepam $10 \mathrm{mg} / \mathrm{kg}$ for INH test and D-AP7 33 ๆmol/ $\mathrm{kg}$ or CGP $378493 \mathrm{mg} / \mathrm{kg}$ for NMDA test.

Table 5. The effects of the different plants on the convulsions and turning behaviour induced in mice by INH, NMDA, PIC, PTZ and STR.

mice against the induced convulsions. $78 \%$ of plants protected both PTZ and STR-induced convulsions. $80.6 \%$ of plants protected both PTZ and PIC-induced convulsions. $80.8 \%$ of plants protected both STR and PIC-induced convulsions. Finally, $66.7 \%$ of plants at the same time protected PTZ, STR and PIC-induced convulsions.

\subsubsection{Plants toxicity}

Datura stramonium, Ricinus communis and Securidaca longepedunculata were also showed to be toxic. Their extract killed animal in $24 \mathrm{~h}$ after their administration to mice.

\section{Discussion and conclusions}

The extracts of twenty one plants (91.3\% of plants) increased the sleeping time induced by sodium thiopental or diazepam. The potentiation of the sleep time suggests the presence of sedative properties in the extracts of these plants (Rakotonirina et al., 2001; Ngo Bum et al., 2009a; 2009b). These sedative properties could be related to the presence of some components in the extracts activating the benzodiazepine, barbiturate and/or GABA 
receptors in the $\mathrm{GABA}_{\mathrm{A}}$ receptor complex (Rang et al., 1999; Bonin \& Orser, 2008; Olkkola \& Ahonen, 2008). Diazepam (benzodiazepine) and sodium thiopental (barbiturate) all bind to the $\mathrm{GABA}_{\mathrm{A}}$ receptor complex. Diazepam potentiates GABA-mediated inhibition via the increase in the affinity of this inhibitory neurotransmitter to its recognition sites within the $\mathrm{GABA}_{\mathrm{A}}$ receptor complex, by increasing the opening frequency of the chloride ion channel which leads to the enhancement of influx of chloride anions into the neuron and subsequent hyperpolarisation (Czapinsky et al., 2005). While sodium thiopental that act on the barbiturate binding site directly gate the chloride ion channel of the $\mathrm{GABA}_{\mathrm{A}}$ receptor complex. The sedative properties found here could explain the use of the twenty one plants in traditional medicine in Africa, particularly in Cameroon in the treatment of insomnia. The first eight more potent plants to induced sedation were: Datura stramonium $>$ Clerodendron thomsoniae $>$ Terminalia mollis $>$ Trichilia emetica $>$ Tetrapleura tétraptera $>$ Annona senegalensis $>$ Securidaca longepedunculata > Hymenocardia acida > Daniellia oliveri. Two plants, Citrus sinenis and Kaya senegalensis did not show sedative properties. The results also showed that 95.6\% of the tested plants possess anticonvulsant properties by inhibiting convulsions induced chemically or electrically. Five plants (Flacourtia indica, Ricinus communis, Securidaca longepedunculata, Senna singueana, Terminalia glaucescens) showed very good anticonvulsant activities against PTZ, PIC or INH induced seizures.

The effect was moderate for the rest of plants. Tetrapleura tetraptera one of the plants studied showed also anticonvulsant properties in fruits (Nwaiwu, 1986; Ojewole, 2005). The antagonism of INH, PTZ- and PIC-induced seizures suggests the interaction of these plants with the GABA-ergic neurotransmission (De Deyn et al., 1992; Doctor et al., 1982; Löscher \& Schmidt, 1988; Salih \& Mustafa, 2008; Perez-Saad \& Buznego, 2008). GABA is the main inhibitory neurotransmitter substance in the brain and is widely implicated in epilepsy. Inhibition of GABA-ergic neurotransmission or activity has been shown to promote and facilitate seizures, while enhancement of GABA-ergic neurotransmission is known to inhibit or attenuate seizures (Gale, 1992; Li-Ping et al., 2008). Moreover, some studies indicated that PTZ diminishes the GABAergic tone (Mcdonald \& Baker. 1977; Ahmadiani, 2003), probably by a competitive antagonist action on the BZD receptors (Rehavi et al., 1982). Correspondingly, drugs that enhance $\mathrm{GABA}_{\mathrm{A}}$-receptor neurotransmission, such as BZDs (White, 1997; Ahmadiani et al., 2003) can block seizures induced by PTZ. PIC is known to be a non competitive GABA antagonist exerting his effect by blocking the chloride channel in the $\mathrm{GABA}_{\mathrm{A}}$ receptor complex. Isoniazide can precipitate convulsions in patients with seizure disorders, and it is regarded as a GABA-synthesis inhibitor (Kale Shubhangi et al., 2010). The antagonism of STR -induced convulsions suggests the presence of anticonvulsant effect through glycine-STR-sensitive receptors (Findlay et al., 2002). Few plants extract antatagonized MES induced convulsions, by probably prolonging neurons sodium channels inactivation (Holmes, 2007). The results show no difference in plants inhibiting convulsions induced by PTZ, PIC and STR. GABA and glycine-STR-sensitive neurotransmission are equally involved. But very few plants produced their anticonvulsant activities by prolonging neurons sodium channels inactivation. Datura stramonium, Ricinus communis and Securidaca longepedunculata were found toxic and therefore they are not suitable to be used to treat people. The toxicity of Ricinus communis could be related to the presence of a very toxic component named ricin (Iwu, 1993). The toxicity of Datura stramonium could be related to its delirants or anticholinergics compounds. 


\section{Conclusion}

The purported anticonvulsant and sedative properties of the medicinal plants are scientifically shown. The ethnopharmacological study on Cameroon anticonvulsant and sedative medicinal plants is accurate in $90 \%$ of cases. A great amount of plants extract interacted through GABA and glycine-STR-sensitive neurotransmissions to inhibit convulsions. Many anticonvulsant plants also possess sedative properties. Twenty one plants possess sedative properties, but only eighteen plants could be used in traditional medicine in Africa in the treatment of insomnia. Eighteen plants possess at least moderate anticonvulsant effects, while five plants possess very good anticonvulsant properties. However only twenty medicinal plants could be used in the treatment of epilepsy. Three plants were found very toxic.

\section{Acknowledgment}

We would like to thank Professor AMVAM ZOLLO Paul Henri, Professor Ngounouno Ismaila, and Dr Megueni Clautilde for their support.

\section{References}

Abbiw, D. K. (1990). Useful plants of Ghana: West African uses of wild and cultivated plants. ISBN 185390437, London, United Kingdom.

Adeyemi, O.O.; Akindele, A.J.; Yemitan, O.K.; Aigbe, F.R. \& Fagbo, F.I. (2010). Anticonvulsant, anxiolytic and sedative activities of the aqueous root extract of Securidaca longepedunculata Fresen. Journal of Ethnopharmacology, Vol.130, No.2, (July, 2010), pp. 191-195, ISSN 0378-8741.

Adjanohoun, J.E.; Ake Assi, L.; Chibon, P.; De Vecchy, H.; Duboze, E.; Eyme, J.; Gassita, J.N.; Goudote, E.; Guinko, S.; Keita, A.; Koudogbo, B.; Le Bras, M.; Mourambou, I.; MveMengome, E.; Nguema, M.G.; Ollome, J.B.; Posso, P. \& Sita, P. (1984). Médecine traditionnelle et pharmacopée: contribution aux études ethnobotaniques et floristiques $d u$ Gabon. Editions A.C.C.T. ISBN 0754-0924, Paris, France.

Adjanohoun, J.E.; Aboubakar, N.; Dramane, K.; Ebot, M.E.; Ekpere, J.A.; Enow- Orok, E.G.; Focho, D.; Gbile, Z.O.; Kamanyi, A.; Kamsu-kom, J.; Keita, A.; Mbenkum, T.; Mbi, C.N.; Mbiele, A.L.; Mbome, L.L.; Mubiru, N.K.; Nancy, W.L.; Nkongmeneck, B.; Satabie, B.; Sofowora, A.; Tamze, V. \& Wirmum, C.K. (1996). Traditional medicine and pharmacopoeia: Contribution to ethnobotanical and floristic studies in Cameroon. Organization of African Unity Scientific, Technical and Research Commission; Centre National de Production de Manuels Scolaires, ISBN 0716-084, Porto-Novo, Benin.

Adzu, B.; Abbah, J.; Vongtau, H. \& Karniyus . (2003). Studies on the use of Cassia singueana in malaria ethnopharmacy, Journal of Ethonopharmacology, Vol.88, No.2-3, (October 2003), pp. 261-267, ISSN: 0378-8741.

Agassounon Djipko Tchibozo, M.; Sanon, S.; Damintoti, S.K.; Toukourou, F.; de Souza, C., Gbeassor, M., (2008). Appréciation des propriétés antiplasmodiale in vitro de Flacourtia flavescens Willd., une plante utilisée dans la médecine traditionnelle en Afrique. 15ème colloque sur la Pharmacopée et la Médecine Traditionnelles Africaines. Vol.15, pp. 156-160. (Décembre 2008), Conseil Africain et Malgache pour l'Enseignement Supérieur (CAMES), Libreville, Gabon. 
Ahmadiani, A.; Mandgary, A. \& Sayyah, M. (2003). Anticonvulsivant Effect of Flutamide on seizures induiced by pentylene tetrazole, involvement of benzodiazepine receptors. Epilepsia, Vol.44, No.5, (May 2003), pp. 629-625, ISSN 1528-1167.

Anete, C.F.; Miriam, E.M. A.; Da Costa, M.L.; Ilza, R.B.; Bras ,H.D.O. \& Cunha, C.D. (1998). Pharmacological Evaluation of Ricinine, a Central Nervous System Stimulant Isolated from Ricinus communis. Pharmacology, Biochemestry and Behavior, Vol.63, No.3, (November 1998), pp. 367-375, ISSN 0091-3057.

Anuradha1, H.; Srikumar, B.N.; Shankaranarayana, Rao, B.S. \& Lakshmana, M. (2008). Euphorbia hirta reverses chronic stress-induced anxiety and mediates its action through the GABAA receptor benzodiazepine receptor-Cl2 channel complex. Journal of Neural transmission, Vol.11, No 3, (September 2008), pp. 35-42, ISSN 1435-1463.

Arbonnier, M. (2000). Arbres, arbustes et lianes des zones sèches d'Afrique de l'Ouest. Mali, Ouagadougou: Centre de Coopération Internationale en Recherche Agronomique pour le développement/Muséum national d'histoire naturelle/Union mondiale pour la nature (CIRAD/MNHN/UICN), IBSN 0209-0914, Paris, France.

Beretz, A.; Haag-Berrurier, M.; Anton, R. (1978). Choix de méthodes pharmacologiques pour l'étude des activités de l'aubépine. Plantes médicinales et phytothérapie, Vol .4, pp. 305314.

Berhaut, J. (1975). Flore illustrée du Sénégal. Dicotylédones. Tome IV Ficoidées à Légumineuses. Government of Senegal, pp. 183-184, 553-555, Dakar, Senegal.

Bernasconi, R.; Klein, M.; Martin, P.; Christen, P.; Hafner, T.; Portet, C. \& Schmutz, M. (1988). Gamma-Vinyl GABA: comparison of neurochemical and anticonvulsant effects in mice. Journal of Neural Transmission, Vol.72, No.3, (October 1988), pp. 213233, ISSN 1435-1463.

Biholong, M. (1986). Contribution à l'étude de la flore du Cameroun: les Astéracées. Thèse de doctorat. Université de Bordeaux III, pp. 10-50, France.

Bonin, R.P. \& Orser, B.A. (2008). GABA $\mathrm{A}_{\mathrm{A}}$ receptor subtypes underlying general anesthesia. Pharmacology Biochemistry and Behavior, Vol.90, No.1, (January 2008), pp. 105-112, ISSN 0091-3057.

Bouquet, A. (1969). Féticheurs et Médecine Traditionnelle au Congo (Brazaville). ORSTOM, IRD, FDI 13972, Mémoire No. 36, pp. 103-127, Montpellier, France.

Boussinesq, M.; Pion, D.S. \& Kamgno, J. (2002). Relationship between Onchocerciasis and epilepsy: a matched case control in the Mbam Valley, Republic of Cameroon. Transaction of the Royal Society of Tropical Medicine and Hygiene, Vol.96, No.5, (September 2002), pp. 537-541, ISSN 0035-9203.

Brenan J.P.M. (1959). Légumineuses (I) sous-famille des Caesalpinioidea dans Flore de l'Afrique orientale tropicale. Agents de la Couronne, Londres, England.

Croucher, J.M.; Collins, J.F. \& Meldrum, B.S. (1982). Anticonvulsant action of excitatory amino acids antagonists. Science, Vol. 216, No. 4548, (May 1982), pp. 899-902, ISSN 1095-9203.

Czapinski, P.; Blaszczyk, B. \& Czuczwar, S.J. (2005). Mechanisms of Action of Antiepileptic Drugs. Current Topics in Medicinal Chemistry, Vol.5, No.1, (January 2005), pp. 3-143, ISSN 1568-0266.

Dalziel, J.M. (1937). The useful plants of West Tropical Africa. The Crown Agency for the Colonies, London, United Kingdom. 
De Deyn, P.P.; D’Hooge, R.; Marescau, B. \& Pei, Y-Q. (1992). Chemical models of epilepsy with some reference to their applicability in the development of anticonvulsants. Epilepsy Research, Vol.12, No.2, (July 1992), pp. 87-110, ISSN 0920-1211.

Dimo, T.; Rakotonirina, S.V.; Tan, P.V.; Azay, J.; Dongo, E. \& Cros, G. (2002). Leaf methanol extract of Bidens pilosa prevents and attenuates the hypertension induced by highfructose diet in wistar rats. Journal of Ethnopharmacology, Vol.83, No. 4, (June 2002), pp. 183-191, ISSN 0378-8741.

Diop, A.G.; Ndiaye, M.; Thiam, A.; et al. (1996). Filière des soins anti-épileptiques en Afrique. Epilepsies, Vol. 10, pp.115-121.

Doctor, S.V.; Costa, L.G. \& Muphur, S.D. (1982). Effect of trimethyltin on chemically induced seizures, Toxicology Letters, Vol.13, No.4, (October 1982), pp. 217-223, ISSN 0378-4274

Dongmo, L.; Ndo, D.; Atchou,G. \& Njamnshi, A. (2000). Epilepsie au Sud-Cameroun: enquête préliminaire dans le village Bilomo. Bulletin de la Société de Pathologie Exotique, Vol.93, No.3, (July 2000), pp. 263-275, ISSN 0037-9085.

Dongmo, L.; Druet-Cabanac, M.; Moyou, S.R.; Zebaze, D.R.; Njamn-shi, A.K.; Sini, V.; Mapoure, N.; Echouffo, T.J.; Djeumen, W.C. \& Ndumbe, P.M. (2004). Cysicercosis and epilepsy: a casec ontrol study in MbamValley, Cameroon. Bulletin de la Société de Pathologie Exotique, Vol. 97, No. 2, (Janvier 2003), p. 105-108, ISSN 0037-9085.

El-Mahmood, A. M; Doughari, J.H. \& Ladan, N. (2008). Antimicrobial screening of stem bark extracts of Vitellaria paradoxa against some enteric pathogenic microorganisms. African Journal of Pharmacy and Pharmacology, Vol. 2, No. 5, (July 2008), pp. 089-094, ISSN: 1996-0816.

Ezugwu, C.O. \& Odoh, U.E. (2003). Anticonvulsant activity of the root extract of Annona senegalensis. Journal of Tropical Medicinal Plants, Vol.4, No.1, (July 2003), pp. 51-55, ISSN 1511-8525.

Findlay, G.S.; Wick, M.J.; Mascia, M.P.; Wallace, D; Millier, G.W.; Harris, R.A. \& Blednov Y.A. (2002). Transgenic expression of a mutant glycine receptor decreases alcohol sensitivity of mice. Journal Pharmacology Experimental Therapeutics, Vol. 300, No. 2, (February 2002), pp. 526-534, ISSN 0022-3565.

Gale K. (1992). GABA and epilepsy: basic concepts from preclinical research. Epelepsia. Vol.33, No.5, (November 1992), pp. S3-S12, ISSN 0013-9580.

Geoffrey, C. \& Kirby, M. (1996). Medicinal plants and the control of protozoa disease with particular reference to malaria. Transaction of the Royal Society of Tropical Medicine and Hygiene, ISSN 0035-9203, London, Great Britain.

Gusman-Gutierez, S.L. \& Navarrete A. (2009). Pharmacological Exploration of the sedative mechanism of hesperidin identified as the Active principle of Citrus sinensis flowers. Planta medica, Vol. 75, No. 4, (February 2009), pp. 295-301, ISSN: 0032-0943.

Holmes, G.L. (2007). Animal model studies application to human patients. Neurology, Vol. 69, No.24, (December 2007), pp. S28-S32, ISSN 0028-3878.

Hutchinson, J. \& Dalziel, J.M. (1958). Flora of West Tropical Africa. Crown Agents, Ed. 2 (Revised by R.W.J. Keay), The Whitefriars Press, London \& Tonbridge, Great Britain.

Iwu, M.M. (1993). Handbook of African Medicinal Plants. CRC Press, ISBN 084934266X, New York, USA.

Kale Shubhangi, S.; Shete Rajkumar, V.; Kore Kakasaheb, J.; Patil Bhaskar, M.; Bhutada Rupesh, N.; Pattankude Vinod, (2010). Anticonvulsant activity of Glycyrrhizic acid in 
mice. International journal of pharmaceutical researches and development, Vol.2, No.2, (December 2010), ISSN 0974 - 9446

Kamgno,J.; Pion,S. \& Boussinesq, M. (2003). Demographic impact of epilepsy in Africa: results of a 10-years cohort study in a rural area of Cameroon. Epilepsia, Vol. 44, No7, (July 2003), pp. 956-963, ISSN 0013-9580.

Lehmann, J.; Hutchison, A.; McPherson, S.E.; Mondadori, C.; Schmutz, M.; Sinton, C.M.; Tsai, C.; Murphy, D.E.; Steel, D.J.; Williams, M.; Cheney, D.L. \& Wood, P.L. (1988). CGS 19755, a selective and competitive N-methyl-D-aspartate-type excitatory amino acid receptor antagonist. Journal of Pharmacology and Experimental Therapeutics Vol.246, No.1, (July 1988), pp. 65-75, ISSN 1521-0103.

Li-Ping, G.; Xian-Yu, S.; Guan-Rong, T.; Kyu-Yun, C. \& Zhe-Shan, Q. (2008). The Synthesis and Anticonvulsant Activity of 1-Substituted-7-Methoxy-1,2,4-Triazolo [4, 3-a] Quinoline; Turkish journal of chemistry, Vol.32, No.2, (April 2008), pp. 181-189, ISSN 1300-0837.

Lompo, M.; Nikiema, J.B.; Guissou, I.P.; Moës, A.J. \& Fontaine, J. (1998). The topical antiinflammatory effects of chloroform extract from Khaya senegalensis stem barks. Phytotherapy Research, Vol.12, No.6, (July 1998), pp. 448-450, ISSN: 0951-418X.

Löscher, W. \& Schmidt, D. (1988). Which animal model should be used in the search for new antiepileptic drugs? A proposal based on the experimental and chemical consideration. Epilepsy Research, Vol. 2, No. 3, (May 1988), pp. 145 -181, ISSN 09201211.

Macdonald, R.L. \& Barker, J.L. (1977). Pentylenetetrazole and penicillin are selective antagonists of GABA-mediated post-synaptic inhibition in cultured mammalian neurons. Nature, Vol.267, No.23, (June 1977), pp. 720-721, ISSN 0028-0836.

Malgras, D. (1992). Arbres et arbustes guérisseurs des savanes maliennes. ACCT, Karthala, ISBN 2865373770, Paris, France.

Mutasa, S.L.; Khan, M. \& Jewers, K. (1990). 7-Methylphyscion and cassiamin A from the root bark of Cassia singueana. Planta Medica. Vol.56, No.2, (April 1990) pp. 244-245, ISSN 0032-0943.

Nazneen, M.; Abdul Mazid; Kundu, J.K.; Bachar, S.C.; Begum, F. \& Datta, B.K. (2009). Protective effects of Flacourtia indica aerial parts extracts against paracetamol-induced hepatotoxicity in rats. Journal of Taibbah University for Science. Vol.2, No.1, (January 2009), pp. 1-6, ISSN 1658-3655.

Nchoji Nkwi,P. \& TiokoNdonko, F. (1989). The Epileptic among the Bamileke of Maham in the Nde Division, West Province of Cameroon. Culture Medicine and Psychiatry, Vol.13, No.4, pp. 437-448, ISSN 0165-005X.

Ngo Bum, E.; Schmutz, M.; Meyer, C.; Rakotonirina, A.; Bopelet, M.; Portet, C.; Jeker, A.; Rakotonirina, S.V.; Olpe, H.R. \& Herrling, P. (2001). Anticonvulsant properties of the methanolic extract of Cyperus articulatus (Cyperaceae). Journal of Ethnopharmacology Vol.76, No.2, (July 2001), pp. 145-150, ISSN: 0378-8741.

Ngo Bum, E.; Taiwe, G.S.; Nkaissa, L.A.; Moto, F.C.O.; Seke, E.P.F.; Hiana, I.R.; Bailabar, T.; Rouyatou; Seyni, P.; Rakotonirina, A. \& Rakotonirina, S.V. (2009a). Validation of anticonvulsant and sedative activity of six medicinal plants. Epilepsy and Behavior, Vol.14, No.3, (March 2009), pp. 454-458, ISSN 1525-5050.

Ngo Bum, E.; Taiwe G.S.; Moto F.C.O.; Ngoupaye G.T.; Nkantchoua G.C.N.; Pelanken M.M.; Rakotonirina S.V. \& Rakotonirina A. (2009b). Anticonvulsant, anxiolytic and sedative 
properties of the roots of Nauclea latifolia Smith in mice. Epilepsy and Behavior, Vol. 15, No. 4, (August 2009), pp. 434-440, ISSN 1525-5050.

Ngounou, E.B.; Quet, F.; Dubreuil, C.M.; Marin, B.; Houinato, D.; Nubukpo, P.; Dalmay, F.; Millogo, A.; Nsengiyumva, G.; Kouna-Ndouongo, P.; Diagana, M.; Ratsimbazafy, V.; Druet-Cabanac, M. \& Preux, P.M. (2007). Épidémiologie de l'épilepsie en Afrique subsaharienne : une revue de la littérature. Cahiers d'études et de recherche francophones/Santé. Vol.16, No.4, (Octobre-novembre-décembre 2006), pp. 225-238, Synthèse.

Njamnshi, A.K.; Bissek, A.C.; Yepnjio, F.N. ; Tabah, E.N.; Angwafor, S.A.; Kuate, C.T.; Déma, F.; Fonsah, J.Y.; Acho, A.; Kepeden, M.N.; Azinwi, Y.H.; Kuwoh, P.B.; Angwafor III, F.F. \& Muna, W.F. (2010). A community survey of knowledge, perceptions, and practice with respect to epilepsy among traditional healers in the Batibo Health District, Cameroon. Epilepsy and behavior, Vol. 17, №1, (January 2010), pp.95-102, PMID: 19932640.

Nwaiwu, J.I. \& Akah, P.A. (1986). Anticonvulsant activity of the volatile oil from the fruit of tetrapleura tetraptera. Journal of Ethnopharmacology Vol.18, No.2, (July 1982), pp. 103107, ISSN: 0378-8741.

Ogundiya, M. O.; Kolapo, A. L.; Okunade, M. B.; Adjumobi, J. A. (2009). Assesment of phytochemical composition and antimicrobial activity of Terminalia glaucescens against some oral pathogens. Electronic journal of environmental, agricultural and food chemistry; Vol.8, No.7, (January 2009), pp. 466-471, ISSN: 1579-4377.

Ojewole, J.A. (2005). Analgesic and anticonvulsant properties of Tetrapleura tetraptera (Taub) (Fabaceae) fruit aqueous extract in mice. Phytotherapy Research, Vol.19, No.12, (December 2005) pp. 1023-1029, ISSN 1099-1573.

Okada, R. \& Negishi, H. (1989). The role of nitrotegmental gabaergic pathway in the propagation of pentylenetetrazol-induced seizures. Brain Research. Vol.480, No.1, (February 1989), pp. 383-387, ISSN 0006-8993.

Olkkola, K.T. \& Ahonen, J. (2008). Midazolam and other benzodiazepines. Handbook of Experimental Pharmacology, Vol.182, No.1, (January 2001), pp. 335-360, ISSN: 03788741.

Palgrave, C.K.; Drumond, R.B.; Eugene, J. \& Palgrave, C.M. (2003). Trees of Southern Africa. Struik Publishers, ISBN 1868723895, Cape Town South Africa.

Pathak, P.; Saraswathy, Vora, A. \& Savai J. (2010). In vitro antimicrobial activity and phytochemical analysis of the leaves of Annona muricata. International Journal of Pharmaceutical Researches and Development, Vol.2, No.5, (July 2010), pp. 1-6, ISSN: 09749446.

Pérez-Saad, H. \& M.T. Buznego (2008). Behavioral and antiepileptic effects of acute administration of the extract of the plant Cestrum nocturnum Lin (lady of the night). Epilepsy and Behaviour, Vol.12, No.3, (April 2008), pp. 366-372, ISSN 1525-5050.

Pousset, J.L. 1989. Plantes médicinales africaines, utilisation pratique. Ellipses, ISBN 2729889183, Paris, France.

Preux, P.M.; Tiemagni,F.; Fodzo,L.; Kandem,P.; Ngouafong, P.; Ndonko,F.; Macharia,W.; Dongmo, L.; Dumas,M. (2000). Antiepileptic therapies in the Mi Province in Cameroon. Epilepsia, Vol.41, No.4. (April 2000), pp. 432-439, ISSN 1528-1167.

Prischich, F.; De Rinaldis, M.; , Flaminia, B.; Egeo, G.; Santori, C.; Zappaterreno, A.; Fattouch, J.; Di Bonaventura, C.; Bada, J.; Gianluca, R.; Pizzuti, A.; Cardona, F.; Sa'a, 
V.V.; Giallonardo, A.T.; D’Erasmo, E.; Pelliccia, A. \& Vanacore, N. (2008). High prevalence of epilepsy in a village in littoral province of Cameroon. Epilepsy Research, Vol. 82, No. 2, (October 2008), pp. 200-210, ISSN 0920-1211.

Rakotonirina, S.V.; Ngo Bum, E.; Rakotonirina, A. \& Bopelet, M. (2001). Sedative properties of the extract of the rhizome of Cyperus articulatus. Fitoterapia, Vol.72, No.1, (September 2001), pp. 22-29, ISSN 0367-0326.

Rang, H.P., Dale, M.M., Ritter, J.M., 1999. Pharmacology. Churchill Livingstone, ISBN 0443059748, New York, USA.

Raponda-Walker, Sillans, R. (1961). Les plantes utiles du Gabon. Edition Sepia, ISBN 2907888692, Libreville, Gabon.

Rehavi, M.; Skolnick, P. \& Paul, .S.M. (1982). Effects of tetrazole derivatives on [3H]diazepam binding: in vitro: correlation with convulsant potency. European Journal of Pharmacoly, Vol.78, No.3, (March 1982), pp. 353-356, ISSN 0014-2999.

Salih, M.A. \& Mustafa, M.M. (2008). A substance in broad beans (Vicia faba) is protective against experimentally induced convulsions in mice. Epilepsy and Behaviour, Vol. 12, No. 1, (January 2008), pp. 25-29, ISSN 1525-5050.

Satyanaraya, V.; Kurupadanam, G.L. \& Srimanaraya, G. (1996). Photochemistry of plants. Fitoterapia. Vol.30, No.4, (July 1996), pp. 1026-1029, ISSN 0367-326X.

Saulnier, P. (1998). Plantes médicinales et soins en Afrique, Edition SEPIA, ISBN : 9782907888929, Saint-Maur, France.

Schmutz, M.; Portet, C.; Jeker, A.; Klebs, K.; Vassout, A.; Allgeier, H.; Heckendorn, R.; Fagg, G.E.; Olpe, H.R. \& Van Riezen, H. (1990). The competitive NMDA receptor antagonists CGP 37849 and CGP 39551 are potent, orally-active anticonvulsants in rodents. Naunyn-Schmiedeberg's Archives of Pharmacology, Vol.342, No.7, (October 1990), pp. 61-66, ISSN 1432-1912.

Seema, Z. (2006). Phytochemical studies on Terminalia glaucescens, Pteleopsis hylodendron and related medical plants. Thèse doctorat Ph.D., Université de Karachi, Pakistan.

Sunday, E.; Atawodi, \& Ogunbusola, F. (2009). Evaluation of Anti trypanosomal Properties of Four Extracts of Leaves, Stem and Root Barks of Prosopis africana in Laboratory Animals. Nigerian for experimental biology Vol.21, No.2, (December 2009), pp. 101-108, ISSN 0795-8080

White, H.S. (1997). New mechanisms of antiepileptic drugs, In: Porter, R., Chadwick, D., (Ed.), 1-30, Butterworth Heinemann, ISBN 1933864168, Boston, USA.

Worapan, S.; Sornkanok, V.; Chuda, C.; Damri, P. \& Siriprapa, A. (2008). Pharmacognostic Investigation of the Leaves of Mentha cordifolia and its DNA Fingerprints. Thai Pharmaceutical and Health Science Journal, Vol. 4, No. 1, (January 2009), pp.9-14. ISSN: 0125-4685. 


\title{
The Potential Role of ATP-sensitive Potassium Channels in Treating Epileptic Disorders
}

\author{
Chin-Wei Huang \\ Department of Neurology, National Cheng Kung University, Tainan \\ Taiwan
}

\section{Introduction}

Despite antiepileptic drug (AED) therapy, epilepsy remains uncontrolled in around one third of patients. The majority of current AEDs fall into two pharmacological classes, those that modulate neuronal voltage-gated sodium and calcium channels and those that modulate neurotransmitters. There is a need for new AEDs with novel mechanisms of action to serve as adjunct therapy for the treatment of intractable epilepsy. Among the diverse molecular targets, to selectively modify the excitability of neurons so that high frequency epileptic firing can be blocked without disturbing normal neuronal activity, potassium is a potential target. Potassium channels play a major role in the control of resting membrane potential, responsiveness to synaptic inputs, spike frequency adaptation and neurotransmitter release. Among them, the important metabolic coupler to electrical activity- ATP-sensitive potassium $\left(\mathrm{K}_{\mathrm{ATP}}\right)$ channels provides a distinct link between the metabolic and electrical state of cells. We have demonstrated the role of $K_{A T P}$ channels in epileptic seizures in diabetic hyperglycemia, providing the direct evidence that increases in extracellular glucose and intracellular ATP attenuate $\mathrm{K}_{\mathrm{ATP}}$ channels, leading to a more excitable state. We also examined the $\mathrm{K}_{\mathrm{ATP}}$ channel agonist mediating neuroprotection in diabetic individuals with status epilepticus. Here, we report how we investigated the diabetic hyperglycemia-related epileptic disorder from clinical observation to experimental studies, and review this potential novel mechanism underlying attenuating epileptic activities by opening $\mathrm{K}_{\mathrm{ATP}}$ channels, especially related to metabolic syndrome.

\section{Epileptic seizures in diabetic hyperglycemia: From clinical observation}

More than 200 million persons worldwide will be diagnosed with diabetes (MandrupPoulsen, 1998). Epileptic seizures with diabetic hyperglycemia (DH) (Maccario et al., 1965; Venna and Sabin, 1981; Huang et al., 2005) are not uncommon and around one-fourth of DH patients have reported seizures (Venna and Sabin, 1981; Singh and Strobos, 1989). In more than half of these patients, seizures reveal previously undiagnosed diabetes (Venna and Sabin, 1981; Tiamkao et al., 2003; Harden et al., 1991).

Most seizures in DH are partial motor seizures (Singh and Strobos, 1989; Loeb, 1974; Tiamkao et al., 2003), while $15 \%$ present as status epilepticus (SE). The level of hyperosmolarity and hyponatremia, accompanied by a wide range of hyperglycemic symptoms, however, are inconsistent (Grant and Warlow, 1985). Previous case reports 
suggest that DH-related epileptic seizures often develop at higher levels of glucose than non-DH-related seizures (Maccario et al., 1965; Venna and Sabin, 1981; Harden et al., 1991).

We conducted a prospective comparative follow-up study, focusing on newly diagnosed unprovoked seizures in adult patients, with and without DH, from 2000 to 2004 (Huang et al., 2008). We found that seizure clustering in initial presentation and in recurrence in the $\mathrm{DH}$ group was significantly higher than that in the non- $\mathrm{DH}$ group. Patients with poor glycemic control (HbA1c >9\%) had significantly higher risk of seizure recurrence and clustering. Thus, DH might play a role in the severity of newly diagnosed adult epileptic seizures. Severe seizures might beget seizures in these patients. $\mathrm{DH}$ should be intensively investigated in adult patients with newly diagnosed seizures and aggressive blood sugar control might benefit seizure treatment in these patients, more than AEDs would.

The pathophysiology of epileptic seizures in DH is probably multi-factorial. Glucose itself could enhance synaptic transmission and propagation, leading to more excitable neurons (Tutka et al., 1998; Gispen and Biessels, 2000) and even epileptic seizures (Schwechter et al., 2003), regardless of the presence of organic lesions. Underlying focal ischemia has also been suggested as having a role in triggering these partial seizures (Singh and Strobos, 1989). Seizure susceptibility even in only moderate degrees of hyperglycemia has been reported in previous studies (Tiamkao et al., 2003; Brick et al., 1989); our study suggests that glucose itself is a pro-convulsant in $\mathrm{DH}$.

In clinical observation, in the DH group, patients with recurrent seizures had more frequent $\mathrm{SE}$ at initial seizure presentation than those without, suggesting potential kindling during poorly controlled DH. In poorly controlled diabetes, neither the continued use of AEDs nor the presence of organic structural lesions affects seizure recurrence. Although simple partial seizures are more common in DH-related epileptic seizures, complex partial seizures, as the second most common in this study, and some rarer presentations, such as reflex, parietooccipital, and sensory seizures (Brick et al., 1989; Huang et al., 2005, 2006, 2010; Lavin, 2005), should be carefully evaluated.

In animal experiments, a higher glucose level have facilitated amygdaloid kindling in rats (Priel et al., 1991) and decreased the time required for 50\% of rats to recover sufficiently from a first maximal electroshock seizure (MES) to be able to have another MES (White et al., 1986). This is compatible with seizure clustering in the DH group observed in this study. This further suggests that kindling may continue and seizures may recur if blood glucose remains high.

Failure to identify the possible association between $\mathrm{DH}$ and seizures is common in clinical practice, potentially leading to inadequate treatment and seizure recurrences. Early recognition of the link will help early diagnosis and treatment, and prevent unnecessary interventions.

\section{Epileptic seizures in experimental animals with diabetic hyperglycemia}

As we all know, glucose plays a major role in metabolism and cerebral functions. However, the effects of hyperglycemia on the central nervous system (CNS) and neuronal excitability (Biessel et al., 1994; Stewart et al., 1999; Gispen and Biessel, 2000) are not fully understood. High glucose concentrations have been associated with a lower seizure threshold in an animal model with a single seizure (Schwechter et al., 2003) and neuronal excitability and seizures are related to rapid glucose utilization and glycolysis (Greene et al., 2003). Experimentally, the correlation between extracellular glucose concentration and excitability 
(seizure) has been established in previous studies (Margineanu et al., 1998; Tutka et al., 1998; Schwechter et al., 2003).

In addition to increasing excitability, higher glycosylated hemoglobin values have been associated with moderate declines in motor speed and psychomotor efficiency (Diabetes Control and Complications Trial/Epidemiology of Diabetes Interventions and Complications Study Research Group et al., 2007). In animal models of diabetes, spatiallearning impairments occur in association with distinct changes in hippocampal synaptic plasticity (Biessels et al., 1998; Kamal et al., 2000). On the other hand, cognitive impairments after SE have been reported in both clinical and experimental studies (Helmstaedter, 2007). Nevertheless, how synaptic plasticity change responds to SE in DH is currently unknown. In addition, it remains to be determined whether $\mathrm{DH}$ exaggerates the cognitive and pathological outcome of SE.

We examined whether SE in rats with $\mathrm{DH}$ caused acute neuronal damage in the hippocampus, and impaired learning and memory, and synaptic plasticity, to determine the behavioral, pathological, and electrophysiological effects of SE on diabetic animals. Adult male Sprague-Dawley rats (150-200 g) were first divided into groups with and without streptozotocin (STZ)-induced diabetes, and then into treatment groups given a normal saline (NS) (STZ-only and NS-only) or a lithium-pilocarpine injection to induce SE (STZ+SE and NS+SE). Serial Morris water-maze test and hippocampal pathology results were examined before and 24 hours after SE. We found the STZ+SE group had a significantly higher percentage of severe seizures and SE-related death and worse learning and memory performances than the other three groups. The STZ+SE group, followed by the NS+SE group, showed the most severe neuronal loss and mossy fiber sprouting in the hippocampal CA3 area.

Tetanic stimulation-induced long-term potentiation (LTP) in a hippocampal slice from these rats was recorded in a multi-electrode dish system (Huang et al., 2006a). LTP was markedly attenuated in the STZ+SE group, followed by the NS+SE group. We also used a simulation model to evaluate intracellular ATP and neuronal excitability and we found increased intracellular ATP concentration promoted action potential firing.

From our animal study, we found that compared with non-diabetic rats, diabetic rats were more susceptible to seizures, had higher SE-related mortality, performed significantly worse on hippocampus-dependent behavioral tests, lost more hippocampal neurons during the acute stage after SE, and exhibited an impaired LTP after SE. This finding suggests the importance of intensively treating hyperglycemia and seizures in diabetic patients with epilepsy (Huang et al., 2009).

SE-induced damage and network reorganization in lithium-pilocarpine-treated rats occurs as a consequence of neuronal loss and SE-induced sprouting (Lehmann et al., 2001). In addition to SE-related excitotoxicity (Curia et al., 2008), DH plus SE may amplify the adverse effects of hyperglycemia on neurons, both the direct effects and the indirect effects, such as diabetic oxidative stress (Vincent et al., 2007; Zupan et al., 2008), microvascular changes (Mraovitch et al., 2005; Qiu et al., 2008), and altered calcium homeostasis (Raza et al., 2004; Biessels et al., 2005). Because neuronal glucose uptake depends on the extracellular concentration of glucose, cellular damage can ensue after persistent episodes of hyperglycemia (Tomlinson and Gardiner, 2008). Diabetes may induce morphological changes in the presynaptic mossy fiber terminals that form excitatory synaptic contacts with the proximal CA3 apical dendrites (Magariños and McEwen, 2000). 
Although impaired, synaptic plasticity is still present in animals with pilocarpine-induced epilepsy (Trudeau et al., 2004). The post-SE water maze analyses showed the STZ+SE group, followed by the NS+SE group, performed significantly worse than the other two groups. These findings again suggest the great negative impact of concomitant $\mathrm{DH}$ and SE on cognition, indicating that these two conditions interact in the brain. It has been suggested (Trudeau et al., 2004) that LTP deficits in diabetes might arise from dysfunction of the Nmethyl-D-aspartate (NMDA) subtype of glutamate receptors in the early stages of the disease. In addition, loss of LTP maintenance in STZ-treated rats has been suggested to be a result of disrupting the calcium-dependent process modulating post-synaptic alpha-amino3-hydroxy-5-methylisoxazole propionic acid (AMPA) receptors (Chabot et al., 1997). The aggravating effect of SE on the glutamatergic system, supported an additional effect of DH on worsening LTP (Pitsch et al., 2007).

One of the empirically based clinical guideline for treating SE is to give glucose immediately (Chen and Wasterlain, 2006). Our study suggests that physicians pay attention to glucose management when encountering concomitant SE and DH. Because seizure clustering and SE in DH are frequently seen in clinical practice, we advocate intensively treating hyperglycemia and seizures in this special population.

\section{Epileptic seizures in diabetic hyperglycemia: A mechanistic view}

The mechanisms underlying hyperglycemia-increased neuronal excitability remains incompletely understood. As a coupling of metabolism to membrane electrical activity, Adenosine triphosphate (ATP)-sensitive $\mathrm{K}^{+}$channels $\left(\mathrm{K}_{\mathrm{ATP}}\right)$ is an important regulator of neuronal excitability and neuroprotection in metabolic stress, such as DH (Liss and Roeper, 2001; Seino and Miki, 2003). The direct connection between the level of electrical activity and intracellular ATP concentration suggests $\mathrm{K}_{\mathrm{ATP}}$ potential for an antiepileptic role. The physiological regulation of $\mathrm{K}_{\mathrm{ATP}}$ during metabolic inhibition involves protein kinase $\mathrm{C}$ mediated $\mathrm{K}_{\mathrm{ATP}}$ internalization to lessen the action potential duration shortening ( $\mathrm{Hu}$ et al., 2003). Whether the epileptic circuit in individuals with $\mathrm{DH}$ involves $\mathrm{K}_{\mathrm{ATP}}$ functional adaptation need further investigation.

$\mathrm{K}_{\mathrm{ATP}}$ exist in many excitable cells, including cardiac myocytes, pancreatic $\beta$ cells, muscle cells, and neurons (Liu et al., 1999, Seino, 1999). In pancreatic $\beta$ cells, these channels are known to couple cellular metabolism to electrical activity by opening and closing as the intracellular ATP/ADP ratio decreases and increases, respectively (Ashcroft and Gribble, 1998). They are octameric complexes composed of four pore-forming units with inward rectifying characteristics (Kir 6.1 or Kir 6.2) and four sulfonylurea (SUR) binding sites (SUR1, SUR2A, or SUR2B) (Shyng and Nichols, 1997), regulated by intracellular ATP as well as pharmacological agents (e.g., diazoxide). These channels are highly responsive to changes in intracellular ATP levels generated during glucose metabolism. Rising levels of intracellular ATP close the $\mathrm{K}_{\text {ATP }}$ leading to depolarization and firing (Rowe et al., 1996; Ashcroft and Gribble, 1998). When the [ATP]/[ADP] ratio decreases, SUR1 and Kir 6.2 interaction reduces the latter's affinity for ATP, thereby opening the $\mathrm{K}_{\mathrm{ATP}}$.

In pancreatic $\beta$ cells, elevation in blood glucose and the closure of $\mathrm{K}_{\mathrm{ATP}}$ trigger events leading to calcium influx, cellular depolarization, and insulin secretion (Miki and Seino, 2005). In the CNS, $\mathrm{K}_{\mathrm{ATP}}$ exist in many tissues, particularly the hippocampus and neocortex (Dunn-Meynell et al., 1998). Except for a similar role in sensing central glucose in hypothalamic glucose-responsive neurons (Miki et al., 2001), they are not involved in 
specific neuroendocrine functions. Therefore, a more general role for these channels, functionally expressed in neurons, needs be investigated. In the brain, cells containing Kir 6.2 mRNA are widely distributed (Dunn-Meynell et al., 1998). A striking overlap with SUR1 mRNA suggests that the Kir 6.2/SUR1 complex is the best candidate for the brain functional $\mathrm{K}_{\text {ATP }}$ (Zawar et al., 1999; Betourne et al., 2009).

Owing to the abundant expression of $\mathrm{K}_{\mathrm{ATP}}$ in the brain (Hicks et al., 1994; Mourre et al., 1990), the activation of $\mathrm{K}_{\mathrm{ATP}}$ during ATP-depleted conditions has become a subject of studies. Mice lacking Kir6.2 (Kir 6.2 (-/-) mice) are vulnerable to hypoxia, exhibiting a reduced threshold for generalized seizure (Yamada et al., 2001). Transgenic mice, overexpressing the SUR1 gene in the forebrain, show a significant increase in the threshold for kainate-induced seizures (Hernandez-Sanchez et al., 2001). However, with excessive extracellular glucose and ATP in the hippocampal neurons, how $\mathrm{K}_{\mathrm{ATP}}$ react is still marginally understood.

We hypothesized increases in extracellular glucose and intracellular ATP would attenuate $\mathrm{K}_{\mathrm{ATP}}$, with cells becoming more depolarized, leading to a more excitable state in hippocampal neurons. Thus, we investigated the effects of higher extracellular glucose on hippocampal $\mathrm{K}_{\mathrm{ATP}}$ channel activities and neuronal excitability by using the cell-attached patch clamp configuration on cultured hippocampal cells (H19-7 cells) and the multielectrode recording system on hippocampal slices. We found that incremental extracellular glucose could attenuate the activities of hippocampal $\mathrm{K}_{\mathrm{ATP}}$ channels. Glucose significantly attenuates $\mathrm{K}_{\mathrm{ATP}}$ channel activity in a concentration-dependent manner, mainly through a decrease in open probabilities. Higher levels of extracellular glucose could enhance neuropropagation which could be attenuated by diazoxide, a $\mathrm{K}_{\mathrm{ATP}}$ channel agonist. Additionally, we found high levels of intracellular ATP, enhanced the firing of action potentials in model neurons. The stochastic increases in intracellular ATP levels also demonstrated an irregular and clustered neuronal firing pattern. Thus, this phenomenon of $\mathrm{K}_{\text {ATP }}$ channel-attenuation could be one of the underlying mechanisms of glucose-related neuronal hyper-excitability and propagation (Huang et al., 2007) (Figure 1).

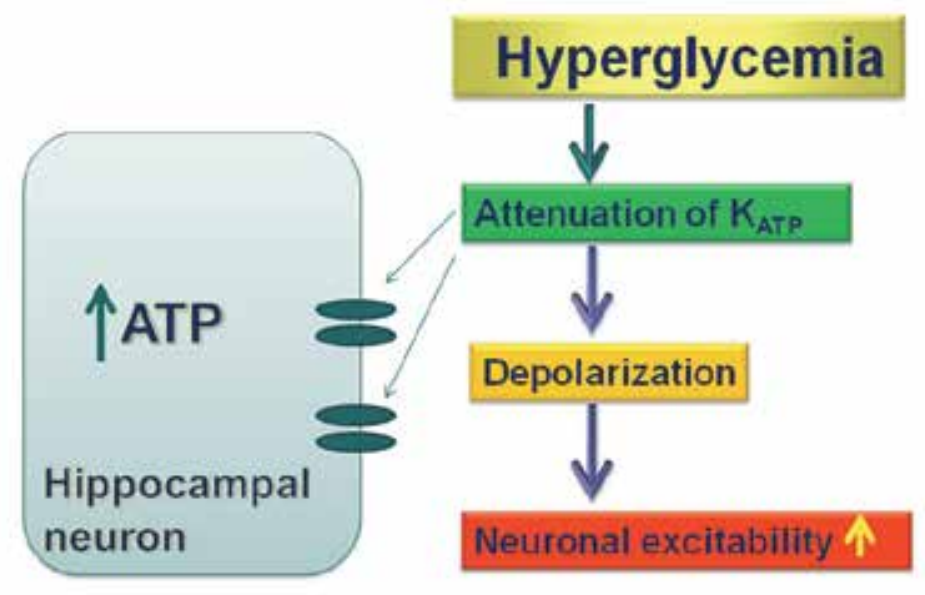

Fig. 1. The scheme of potential role of $\mathrm{K}_{\mathrm{ATP}}$ underlying epileptic seizures in diabetic hyperglycemia. 
From our study, we found glucose could enhance propagation through inhibition of $\mathrm{K}_{\mathrm{ATP}}$ channel activity. The single-channel conductance, open-time, channel-bursting, ATPsensitivity and voltage-insensitivity observed in these H19-7 cells were nearly identical to those described in native pancreatic $\beta$ cells (Kir 6.2/SUR1) (Mukai et al., 1998). Despite the heterogeneous expression profiles of $\mathrm{K}_{\mathrm{ATP}}$ channel subunits reported in hippocampal pyramidal neurons (Zawar et al., 1999), our study emphasized the role of $\beta$-cell type $\mathrm{K}_{\mathrm{ATP}}$ channel in the hippocampus.

The effect of glucose on propagation has been suggested to be related to post-synaptic NMDA receptor activities (Abulrob et al., 2005). We implies the novel role of $K_{A T P}$ channels. The increment in glucose leads to attenuation of $\mathrm{K}_{\mathrm{ATP}}$ channel activities which would enhance field effects of EPSP, potentially caused by electrotonic spread of depolarization. Both pre- and post-synaptic $\mathrm{K}_{\mathrm{ATP}}$ channels were involved in the electrical coupling effects in neurons (Matsumoto et al., 2002). Our study could support the role of post-synaptic $\mathrm{K}_{\mathrm{ATP}}$ channel in neuropropagation, as there were more dominant effects on fEPSP with a relatively limited effect on pre-synaptic fiber volley and paired-pulse facilitation, in the presence of high glucose concentration.

The concentration-dependent attenuation of glucose on $\mathrm{K}_{\mathrm{ATP}}$-channel activity also aids in understanding the higher seizure susceptibility in higher degrees of hyperglycemia. Moreover, the stochastic simulation in this study suggested that in a state of intracellular ATP fluctuation, the neuronal firing pattern would show irregularity. This could be in parallel with the clinical situation where hyperglycemia-related seizures might develop as a result of paroxysmal action potential development in a steady state of hyperglycemia.

\section{The therapeutic point-of-view on epileptic seizures in metabolic syndrome}

As we investigated, the important metabolic couplers to electrical activity, $\mathrm{K}_{\mathrm{ATP}}$ are potential mechanistic candidates when we treat these seizures (Figure 1). For clinical application, we started from the current available AEDs and we found there have been a few reports concerning the effects of Pregabalin (PGB), a newer AED, on some modulating effects on voltage-gated potassium channel (McClelland et al., 2004). Gabapentin-lactam, the derivative of gabapentin, has been found to exert an opening effect on $\mathrm{K}_{\mathrm{ATP}}$ on the mitochondria (Pielen et al., 2004), which is a role in neuroprotection, added to the reduction of neuronal excitability. Moreover, it has been demonstrated that gabapentin can inhibit $\mathrm{K}^{+}-$ evoked $\left[{ }^{3} \mathrm{H}\right]$-noradrenaline release through the activation of $\mathrm{K}_{\mathrm{ATP}}$ in both rat hippocampal and human neocortical cortex (Freiman et al., 2001). However, studies regarding the effects of PGB on $\mathrm{K}_{\text {ATP }}$ are still lacking.

We thus conducted an in vitro cellular study to investigate the effect of PGB on the activity of $\mathrm{K}_{\mathrm{ATP}}$ present in H19-7 neurons. The inside-out configuration of the patch-clamp technique was employed to investigate $\mathrm{K}_{\mathrm{ATP}}$ channel activities. Interestingly, PGB significantly opened these $\mathrm{K}_{\mathrm{ATP}}$ channel activities in a concentration-dependent fashion with an $\mathrm{EC}_{50}$ value of 18 $\mu \mathrm{M}$. There was a significant increase in the mean open-life time of $\mathrm{K}_{\mathrm{ATP}}$ channels in the presence of PGB.

This study suggests that in differentiated hippocampal neuron-derived H19-7 cells, the opening effect on $\mathrm{K}_{\mathrm{ATP}}$ channels could be one of PGB's underlying mechanisms in the reduction of neuronal excitability (Huang et al., 2006b). It's a novel finding regarding the mechanisms of PGB. Of interest, PGB applied to the intracellular surface of the excised 
patches is able to activate $\mathrm{K}_{\mathrm{ATP}}$ channels in these cells, suggesting that its binding side could be primarily on the intracellular leaflet.

The opening of $K_{A T P}$ channels has been noted as neuroprotective, especially the $\beta$-cell-type $\mathrm{K}_{\text {ATP }}$ channels comprised of Kir6.2 and SUR1 (Yamada et al., 2001). Therefore, it is conceivable that the activation of $\mathrm{K}_{\mathrm{ATP}}$ channels by PGB is anti-epileptic and potentially neuroprotective. PGB has been shown to rapidly penetrate the blood-brain barrier in preclinical animal studies (Ben-Menachem, 2004). In PGB treatment $(600 \mathrm{mg} / \mathrm{d})$ for epilepsy, the usual therapeutic concentration range is around $2.8-8.2 \mathrm{mg} / \mathrm{L}(\fallingdotseq 15-43 \mu \mathrm{M})$ at steady state (Berry et al., 2005). From our study, the $\mathrm{EC}_{50}$ for PGB in opening $\mathrm{K}_{\mathrm{ATP}}$ channel activities is around $18 \mu \mathrm{M}$. From this point, it appears that the clinically relevant concentration would be similar to the concentration noted in our study.

Although we have shown (Huang et al., 2007) that, in in vitro hippocampal neurons, $\mathrm{K}_{\mathrm{ATP}}$ agonists lead to membrane hyperpolarization and attenuate action-potential firing when extracellular glucose concentrations are high, there were no in vivo studies on whether $\mathrm{K}_{\mathrm{ATP}}$ agonists protect against seizure severity and consequent SE-induced hippocampal damage in rats with DH. In addition, the functional relationship between Kir 6.2 and SUR1 in DHrelated seizures remains unclear. We hypothesized diazoxide is protective in diabetic rats with SE, and, if so, whether the opening of $\mathrm{K}_{\mathrm{ATP}}$ mediates this protection. $\mathrm{K}_{\mathrm{ATP}}$ openers, including diazoxide, protect beta cells (Kir 6.2/SUR1) and preserve human and rat islets in high concentrations of glucose (Björklund et al., 2004; Maedler et al., 2004). Molecular and electrophysiological studies (Miki et al., 2001; Bancila et al., 2005; Sun et al., 2006; Huang et al., 2006b) report that Kir 6.2/SUR1 channels, like pancreatic beta cells, seem to be the dominant $\mathrm{K}_{\mathrm{ATP}}$ isoform in the brain. It is thus reasonable to hypothesize that diazoxide protects CNS neurons.

In this study, adult male Sprague-Dawley rats (150-200g) were divided into two groups: the STZ-induced diabetes (STZ) group and the normal saline (NS) group. Both groups were treated with either diazoxide (DZX, $15 \mathrm{mg} / \mathrm{kg}$, i.v.) (STZ+DZX, NS+DZX) or vehicle $(\mathrm{STZ}+\mathrm{V}, \mathrm{NS}+\mathrm{V})$ before lithium-pilocarpine-induced SE. We evaluated seizure susceptibility, severity, and mortality. The rats underwent Morris water-maze tests and hippocampal histopathology analyses 24 hours post-SE. Similar to previous studies, a multi-electrode recording system was used to study fEPSP. Seizures were less severe, post-SE learning and memory were better, and neuron loss in the hippocampal CA3 area was lower in the $\mathrm{STZ}+\mathrm{DZX}$ than the STZ+V group. In contrast, seizure severity, post-SE learning and memory, and hippocampal CA3 neuron loss were comparable in the NS+DZX and NS+V groups. fEPSP was lower in the STZ+DZX but not in the NS+DZX group. In addition, RNA interference (RNAi) to knockdown Kir 6.2 in a hippocampal cell line was used to evaluate the effect of diazoxide, in the presence of high concentration of ATP. The RNAi study confirmed that diazoxide, with its $\mathrm{K}_{\mathrm{ATP}}$-opening effects, could counteract the $\mathrm{K}_{\mathrm{ATP}}$-closing effect by high dose ATP. We conclude that, by opening $\mathrm{K}_{\mathrm{ATP}}$, diazoxide protects against SEinduced neuron damage during $\mathrm{DH}$ (Huang et al., 2010).

We showed in vivo and in vitro evidence that diazoxide indeed protected STZ diabetic rats against SE-induced hippocampal damage. There are reports (Mattia et al., 1994; Yamada et al., 2001; Soundarapandian et al., 2007) that modulated $\mathrm{K}_{\text {ATP }}$ may alter the seizure threshold and epileptiform activity in hippocampal slices in rats. Nevertheless, this class of compounds has not yet been generally effective in some animal models, such as the maximal electroshock model, the kindling model (Wickenden, 2002), and in our NS rats. 
[3H] glyburide binding to SUR receptors in the brain appears to be generally upregulated in the state of hyperglycemia (Levin and Dunn-Meynell, 1998). In rats with DH, diazoxide opened $\mathrm{K}_{\mathrm{ATP}}$, which were frequently attenuated in a state of high extracellular glucose concentration (Huang et al., 2007). Diazoxide reduced glutamate release by opening presynaptic $\mathrm{K}_{\mathrm{ATP}}$ Kir 6.2/SUR1 channels (Bancila et al., 2004). As diazoxide activates $\mathrm{K}_{\mathrm{ATP}}$ by interacting with the SUR1 subunit (transmembrane domain 1 and nucleotide binding domain 1) (Nichols, 2006), opening $\mathrm{K}_{\mathrm{ATP}}$ is potentially beneficial in a seizure during hyperglycemia.

The hippocampus is rich in Kir 6.2/SUR1-based channels (Thomzig et al., 2005; Sun et al., 2006). Hippocampal $K_{\text {ATP }}$ are involved in processing new information at the mossy fiber CA3 synapses (Quinta-Ferreira and Matias, 2005). Reports (Zarrindast et al., 2006; Betourne et al., 2009) on the effect of diazoxide on contextual memory, however, are inconsistent. During learning, as the energy demand increases, mossy fiber $\mathrm{K}_{\mathrm{ATP}}$ may sense a rise in intracellular ATP, which closes the $\mathrm{K}_{\mathrm{ATP}}$ and increases glutamate release. Conversely, $\mathrm{K}_{\mathrm{ATP}}$ that open immediately after intense electrical activity may also protect CA3 cells from glutamate-mediated excitotoxicity (Betourne et al., 2009). Based on our water-maze and pathology results, diazoxide -induced opening of $\mathrm{K}_{\mathrm{ATP}}$ counteracted SE-related excitotoxicity in STZ rats.

\section{Conclusion}

Diabetic hyperglycemia might aggravate seizures. An aggressive search for diabetic hyperglycemia and intensive control of glucose in new onset seizures are helpful in management. The outcome of seizures is probably more deteriorating in diabetic patients with epilepsy. Because seizure clustering and status epilepticus in diabetic hyperglycemia are frequently seen in clinical practice, we advocate intensively treating hyperglycemia and seizures in this special population.

Our study provides more direct mechanistic evidence that increments of central neuron excitability, in a state of high glucose levels, can be attributed to $\mathrm{K}_{\text {ATP }}$ channel activity attenuation. $\mathrm{K}_{\mathrm{ATP}}$ agonists are worth investigating as treatments for epileptic seizures in diabetic hyperglycemia.

\section{Acknowledgements}

The study is supported by grants from the National Science Council (NSC-98-2314-B-006042-MY2), Taiwan.

\section{References}

Abulrob, A., Tauskela, J.S., Mealing, G., Brunette, E., Faid, K. \& Stanimirovic, D. (2005). Protection by cholesterol-extracting cyclodextrins: a role for N-methyl-D-aspartate receptor redistribution. J Neurochem 92:1477-1486.

Ashcroft, F.M. \& Gribble, F.M. (1998). Correlating structure and function in ATP-sensitive K+ channels. Trends Neurosci 21:288-294.

Bancila, V., Nikonenko, I., Dunant, Y. \& Bloc, A. (2004). Zinc inhibits glutamate release via activation of pre-synaptic $\mathrm{K}$ channels and reduces ischaemic damage in rat hippocampus. J Neurochem 90:1243-1250. 
Bancila V, Cens T, Monnier D, Chanson F, Faure C, Dunant Y, Bloc A (2005) Two SUR1specific histidine residues mandatory for zinc-induced activation of the rat KATP channel. J Biol Chem 280:8793-8799.

Ben-Menachem E. Pregabalin pharmacology and its relevance to clinical practice. Epilepsia 2004;45:S13-8.

Berry D, Millington C. Analysis of pregabalin at therapeutic concentrations in human plasma/serum by reversed-phase HPLC. Ther Drug Monit 2005;27:451-6.

Betourne A, Bertholet AM, Labroue E, Halley H, Sun HS, Lorsignol A, Feng ZP, French RJ, Penicaud L, Lassalle JM, Frances B (2009) Involvement of hippocampal CA3 K(ATP) channels in contextual memory. Neuropharmacology 56:615-625.

Biessels GJ, Kamal A, Urban IJ, Spruijt BM, Erkelens DW, Gispen WH (1998) Water maze learning and hippocampal synaptic plasticity in streptozotocin-diabetic rats: effects of insulin treatment. Brain Res 800:125-135.

Biessels GJ, Ter Laak MP, Kamal A, Gispen WH (2005) Effects of the Ca ${ }^{2+}$ antagonist nimodipine on functional deficits in the peripheral and central nervous system of streptozotocin-diabetic rats. Brain Res 1035:86-93.

Björklund A, Bondo Hansen J, Falkmer S, Grill V (2004) Openers of ATP-dependent K+channels protect against a signal-transduction-linked and not freely reversible defect of insulin secretion in a rat islet transplantation model of Type 2 diabetes. Diabetologia 47:885-891.

Brick JF, Gutrecht JA, Ringel RA (1989) Reflex epilepsy and nonketotic hyperglycemia in the elderly: a specific neuroendocrine syndrome. Neurology 39: 394-399.

Chabot C, Massicotte G, Milot M, Trudeau F, Gagné J (1997) Impaired modulation of AMPA receptors by calcium-dependent processes in streptozotocin-induced diabetic rats. Brain Res 768:249-256.

Chen JW, Wasterlain CG (2006) Status epilepticus: pathophysiology and management in adults. Lancet Neurol 5:246-256.

Covolan L, Mello LE (2000) Temporal profile of neuron injury following pilocarpine or kainic acid-induced status epilepticus. Epilepsy Res 39:133-152.

Curia G, Longo D, Biagini G, Jones RS, Avoli M (2008) The pilocarpine model of temporal lobe epilepsy. The pilocarpine model of temporal lobe epilepsy. J Neurosci Methods 172:143-157.

Diabetes Control and Complications Trial/Epidemiology of Diabetes Interventions and Complications Study Research Group, Jacobson AM, Musen G, Ryan CM, Silvers N, Cleary P, Waberski B, Burwood A, Weinger K, Bayless M, Dahms W, Harth J (2007) Long-term effect of diabetes and its treatment on cognitive function. $\mathrm{N} \mathrm{Engl} \mathrm{J}$ Med 356:1842-1852.

Dunn-Meynell AA, Rawson NE, Levin BE (1998) Distribution and phenotype of neurons containing the ATP-sensitive $\mathrm{K}+$ channel in rat brain. Brain Res 814:41-54.

Freiman TM, Kukolja J, Heinemeyer J, Eckhardt K, Aranda H, Rominger A, Dooley DJ, Zentner J, Feuerstein TJ (2001) Modulation of $\mathrm{K}^{+}$-evoked [3H]-noradrenaline release from rat and human brain slices by gabapentin: involvement of $\mathrm{K}_{\mathrm{ATP}}$ channels. Naunyn-Schmiedeberg's Arch Pharmacol 363:537-542.

Gispen WH, Biessels GJ (2000) Cognition and synaptic plasticity in diabetes mellitus. Trends Neurosci 23:542-549. 
Grant C, Warlow C (1985) Focal epilepsy in diabetic non-ketotic hyperglycaemia. Br. Med. J 290:1204-1205.

Greene AE, Todorova MT, Seyfried TN (2003) Perspectives on the metabolic management of epilepsy through dietary reduction and elevation of ketone bodies. J Neurochem 86:529-537.

Harden CL, Rosenbaum DH, Daras M (1991) Hyperglycemia presenting with occipital seizures. Epilepsia 32:215-220.

Helmstaedter C (2007) Cognitive outcome of status epilepticus in adults. Epilepsia S48: 85-90.

Hernandez-Sanchez C, Basile AS, Fedorova I, Arima H, Stannard B, Fernandez AM, Ito Y, LeRoith D (2001) Mice transgenically overexpressing sulfonylurea receptor 1 in forebrain resist seizure induction and excitotoxic neuron death. Proc Natl Acad Sci USA 98:3549-3554.

Hicks GA, Hudson AL, Henderson G (1994) Localization of high affinity $\left[{ }^{3} \mathrm{H}\right]$ glibenclamide binding sites within the substantia nigra zona reticulata of the rat brain. Neuroscience 61:285-292.

Hu K, Huang CS, Jan YN, Jan LY (2003) ATP-sensitive potassium channel traffic regulation by adenosine and protein kinase C. Neuron 38:417-432.

Huang CW, Hsieh YJ, Pai MC, Tsai JJ, Huang CC (2005) Non-ketotic hyperglycemia-related epilepsia partialis continua with ictal unilateral parietal hyperperfusion. Epilepsia 46:1843-1844.

Huang CW, Hsieh YJ, Tsai JJ, Huang CC (2006a) The effect of lamotrigine on field potentials, propagation and long-term potentiation in rat prefrontal cortex in multi-electrode recording. J Neurosci Res 83:1141-1150.

Huang CW, Huang CC, Wu SN (2006b) The Opening Effect of Pregabalin on ATP-Sensitive Potassium Channels in Differentiated Hippocampal Neuron-Derived H19-7 Cells. Epilepsia 47:720-726.

Huang CW, Huang CC, Cheng JT, Tsai JJ, Wu SN (2007) Glucose and hippocampal neuronal excitability: The role of ATP-sensitive potassium channels. J Neurosci Res 85:14681477.

Huang CW, Tsai JJ, Ou HY, Wang ST, Cheng JT, Wu SN, Huang CC (2008a) Diabetic Hyperglycemia is associated with the severity of epileptic seizures in adults. Epilepsy Res 79:71-77.

Huang CW, Cheng JT, Tsai JJ, Wu SN, Huang CC (2009) Diabetes aggravates epileptic seizures and status epilepticus-induced hippocampal damage. Neurotox Res 15:7181.

Huang CW, Wu SN, Cheng JT, Tsai JJ, Huang CC (2010). Diazoxide reduces status epilepticus neuron damage in diabetes. Neurotox Res 17:305-316.

Kamal A, Biessels GJ, Duis SE, Gispen WH (2000) Learning and hippocampal synaptic plasticity in streptozotocin-diabetic rats: interaction of diabetes and ageing. Diabetologia 43:500-506.

Lavin PJ (2005) Hyperglycemic hemianopia: a reversible complication of non-ketotic hyperglycemia. Neurology 65:616-619.

Lehmann TN, Gabriel S, Eilers A, Njunting M, Kovacs R, Schulze K, Lanksch WR, Heinemann U (2001) Fluorescent tracer in pilocarpine-treated rats shows widespread aberrant hippocampal neuronal connectivity. Eur J Neurosci 14:83-95. 
Levin BE, Dunn-Meynell AA (1998) Effect of streptozotocin-induced diabetes on rat brain sulfonylurea binding sites. Brain Res Bull 46:513-518.

Liss B, Roeper J (2001) A role for neuronal K(ATP) channels in metabolic control of the seizure gate. Trends Pharmacol Sci 22:599-601.

Liu M, Seino S, Kirchgessner AL (1999) Identification and characterization of glucoresponsive neurons in the enteric nervous system J Neurosci 19:10305-10317.

Loeb JN (1974) The hyperosmolar state. N Engl J Med 290:1184-1187.

Maedler K, Størling J, Sturis J, Zuellig RA, Spinas GA, Arkhammar PO, Mandrup-Poulsen T, Donath MY (2004) Glucose- and interleukin-1beta-induced beta-cell apoptosis requires Ca2+ influx and extracellular signal-regulated kinase (ERK) 1/2 activation and is prevented by a sulfonylurea receptor 1/inwardly rectifying $\mathrm{K}+$ channel 6.2 (SUR/Kir6.2) selective potassium channel opener in human islets. Diabetes 53:17061713.

Mattia D, Nagao T, Rogawski MA, Avoli M (1994) Potassium channel activators counteract anoxic hyperexcitability but not 4-aminopyridine-induced epileptiform activity in the rat hippocampal slice. Neuropharmacology 33:1515-1522.

Miki T, Liss B, Minami K, Shiuchi T, Saraya A, Kashima Y, Horiuchi M, Ashcroft F, Minokoshi Y, Roeper J, Seino S (2001) ATP-sensitive $\mathrm{K}^{+}$channels in the hypothalamus are essential for the maintenance of glucose homeostasis. Nat Neurosci 4:507-512.

Miki T, Seino S (2005) Roles of $\mathrm{K}_{\mathrm{ATP}}$ channels as metabolic sensors in acute metabolic changes. J Mol Cell Cardiol 38:917-925.

Monnier L, Mas E, Ginet C, Michel F, Villon L, Cristol JP, Colette C (2006) Activation of oxidative stress by acute glucose fluctuations compared with sustained chronic hyperglycemia in patients with type 2 diabetes. JAMA 295:1681-1687.

Mukai E, Ishida H, Kato S, Tsuura Y, Fujimoto S, Ishida-Takahashi A, Horie M, Tsuda K, Seino Y (1998) Metabolic inhibition impairs ATP-sensitive $\mathrm{K}^{+}$channel block by sulfonylurea in pancreatic beta-cells. Am J Physiol 274:E38-44.

Pielen A, Kirsch M, Hofmann H, Feuerstein TJ, Lagreze WA (2004) Retinal ganglion cell survival is enhanced by gabapentin-lactam in vitro: evidence for involvement of mitochondrial $\mathrm{K}_{\mathrm{ATP}}$ channels. Graefes Arch Clin Exp Ophthalmol 242:240-244.

Pitsch J, Schoch S, Gueler N, Flor PJ, van der Putten H, Becker AJ (2007) Functional role of mGluR1 and mGluR4 in pilocarpine-induced temporal lobe epilepsy. Neurobiol Dis 26:623-633.

Priel MR, Bortolotto ZA, Cavalheiro EA (1991) Effects of systemic glucose injection on the development of amygdala kindling in rats. Behav Neural Biol 56: 314-318.

Qiu C, Cotch MF, Sigurdsson S, Garcia M, Klein R, Jonasson F, Klein BE, Eiriksdottir G, Harris TB, van Buchem MA, Gudnason V, Launer LJ (2008) Retinal and cerebral microvascular signs and diabetes: the age, gene/environment susceptibilityReykjavik study. Diabetes 57:1645-1650.

Quinta-Ferreira ME, Matias CM (2005) Tetanically released zinc inhibits hippocampal mossy fiber calcium, zinc and synaptic responses. Brain Res 1047:1-9.

Raza M, Blair RE, Sombati S, Carter DS, Deshpande LS, DeLorenzo RJ (2004) Evidence that injury-induced changes in hippocampal neuronal calcium dynamics during epileptogenesis cause acquired epilepsy. Proc Natl Acad Sci USA 101:17522-17527. 
Rowe IC, Treherne JM, Ashford ML (1996) Activation by intracellular ATP of a potassium channel in neurones from rat basomedial hypothalamus. J Physiol 490:97-113.

Schwechter EM, Veliskova J, Velisek L (2003) Correlation between extracellular glucose and seizure susceptibility in adult rats. Ann Neurol 53:91-101.

Seino S (1999) ATP-sensitive potassium channels: a model of heteromultimeric potassium channel/receptor assemblies. Annu Rev Physiol 61:337-362.

Seino S, Miki T (2003) Physiological and pathophysiological roles of ATP-sensitive $\mathrm{K}^{+}$ channels. Prog Biophys Mol Biol 81:133-176.

Shyng S, Nichols CG (1997) Octameric stoichiometry of the KATP channel complex. J Gen Physiol 110:655-664.

Singh BM, Strobos RJ (1989) Epilepsia partialis continua associated with nonketotic hyperglycemia: clinical and biochemical profile of 21 patients. Ann Neurol 8:155-160.

Soundarapandian MM, Wu D, Zhong X, Petralia RS, Peng L, Tu W, Lu Y (2007) Expression of functional Kir6.1 channels regulates glutamate release at CA3 synapses in generation of epileptic form of seizures. J Neurochem 103:1982-1988.

Stewart R, Liolista D (1999) Type 2 diabetes mellitus, cognitive impairment and dementia. Diabet Med 16:93-112.

Sun HS, Feng ZP, Miki T, Seino S, French RJ (2006) Enhanced neuronal damage after ischemic insults in mice lacking Kir6.2-containing ATP-sensitive Kt channels. J Neurophysiol 95:2590-2601.

Thomzig A, Laube G, Pruss H, Veh RW (2005) Pore-forming subunits of K-ATP channels, Kir6.1 and Kir6.2, display prominent differences in regional and cellular distribution in the rat brain. J Comp Neurol 484:313-330.

Tiamkao S, Pratipanawatr T, Tiamkao S, Nitinavakarn B, Chotmongkol V, Jitpimolmard S (2003) Seizures in nonketotic hyperglycaemia. Seizure 12:409-410.

Tomlinson DR, Gardiner NJ (2008) Glucose neurotoxicity. Nat Rev Neurosci 9:36-45.

Trudeau F, Gagnon S, Massicotte G (2004) Hippocampal synaptic plasticity and glutamate receptor regulation: influences of diabetes mellitus. Eur J Pharmacol 490:177-186.

Tutka P, Sawiniec J, Kleinrok Z (1998) Experimental diabetes sensitizes mice to electricaland bicuculline-induced convulsions. Pol J Pharmacol 50:92-93.

Venna N, Sabin TD (1981) Tonic focal seizures in nonketotic hyperglycemia of diabetes mellitus. Arch Neurol 38:512-514.

White HS, Woodbury DM, Chen CF, Kemp JW, Chow SY, Yen-Chow YC (1986) Role of glial cation and anion transport mechanisms in etiology and arrest of seizures. Adv Neurol 44:695-712.

Yamada K, Ji JJ, Yuan H, Miki T, Sato S, Horimoto N, Shimizu T, Seino S, Inagaki N (2001) Protective role of ATP-sensitive potassium channels in hypoxia-induced generalized seizure. Science 292:1543-1546.

Zarrindast MR, Ebrahimi M, Khalilzadeh A (2006) Influence of ATP-sensitive potassium channels on lithium state-dependent memory of passive avoidance in mice. Eur J Pharmacol 550:107-111.

Zawar C, Plant TD, Schirra C, Konnerth A, Neumcke B (1999) Cell-type specific expression of ATP-sensitive potassium channels in the rat hippocampus. J Physiol 514:327-341.

Zupan G, Pilipović K, Hrelja A, Peternel S (2008) Oxidative stress parameters in different rat brain structures after electroconvulsive shock-induced seizures. Prog Neuropsychopharmacol Biol Psychiatry 32:771-777. 

